\title{
Study of the CP Symmetry Violation in Partially Reconstructed B0 ---> D* Pi Decays With the BaBar Detector
}

\author{
Marie Legendre \\ Stanford Linear Accelerator Center \\ Stanford University \\ Stanford, CA 94309
}

SLAC-Report-803

Prepared for the Department of Energy

under contract number DE-AC02-76SF00515

Printed in the United States of America. Available from the National Technical Information Service, U.S. Department of Commerce, 5285 Port Royal Road, Springfield, VA 22161. 
This document, and the material and data contained therein, was developed under sponsorship of the United States Government. Neither the United States nor the Department of Energy, nor the Leland Stanford Junior University, nor their employees, nor their respective contractors, subcontractors, or their employees, makes an warranty, express or implied, or assumes any liability of responsibility for accuracy, completeness or usefulness of any information, apparatus, product or process disclosed, or represents that its use will not infringe privately owned rights. Mention of any product, its manufacturer, or suppliers shall not, nor is it intended to, imply approval, disapproval, or fitness of any particular use. A royalty-free, nonexclusive right to use and disseminate same of whatsoever, is expressly reserved to the United States and the University. 


\title{
UNIVERSITÉ DENIS DIDEROT
}

\section{U.F.R. SCIENTIFIQUE DE PARIS VII}

\section{Étude de la violation de la symétrie $C P$ dans les désintégrations $B^{0} \rightarrow D^{*} \pi$ partiellement reconstruites avec le détecteur $B_{A} B_{A R}$}

\author{
THÈSE \\ présentée et soutenue publiquement le $1^{\text {er }}$ avril 2005 \\ pour l'obtention du
}

Grade de Docteur en Physique de l'Université Paris VII

par

Marie Legendre

Composition du jury

$\begin{array}{ll}\text { Rapporteurs : } & \text { Livio Lanceri } \\ & \text { Renaud Le Gac } \\ \text { Examinateurs : } & \text { Jérôme Charles (invité) } \\ & \text { Lydia Fayard } \\ & \text { François Le Diberder } \\ & \text { Bruno Mansoulié } \\ & \\ \text { Directeur de thèse : } & \text { Marco Zito }\end{array}$


Mis en page avec la classe thloria. 


\section{Remerciements}

Je remercie Pascal Debu, Bruno Mansoulié, Michel Spiro et Jean Zinn-Justin pour m'avoir accueillie pendant ma thèse au sein du Service de Physique des Particules du CEA Saclay.

Je voudrais commencer par remercier chaleureusement mon directeur de thèse Marco Zito pour son aide et pour ses conseils précieux au long de ma thèse. Merci de m'avoir permis de travailler sur un sujet passionnant et qui s'est révélé être très prometteur. Je remercie également Sergey Ganzhur pour la fructueuse collaboration que nous avons eue et qui nous a permis de mener à bien l'analyse. Merci également à Abi Soffer pour son aide précieuse et sa gentillesse. Je suis également très reconnaissante à Roy Aleksan. Je remercie enfin tout le groupe d'analyse IHBD, au sein duquel il a été très agréable de travailler.

Je remercie Jérôme Charles, Lydia Fayard, Livio Lanceri, François Le Diberder, Renaud Le Gac et Bruno Mansoulié pour avoir accepté de faire partie de mon jury de thèse. Je remercie particulièrement Livio et Renaud pour avoir accepté d'être rapporteur, tâche dont ils se sont acquittés avec beaucoup de sérieux et de gentillesse.

Je tiens à remercier l'ensemble du groupe BABAR de Saclay : Sandrine Emery, André Gaidot, Sergey Ganzhur, Pierre-François Giraud, Gautier Hamel de Monchenault, Witold Kozanecki, Benjamin Mayer, Grégory Schott, Georges Vasseur, Christophe Yèche et Marco Zito. Merci Georges pour ta gentillesse et ton soutien permanent. Merci Gautier pour tout et en particulier pour m'avoir fait profiter de tes nombreuses connaissances et pour m'avoir conseillée judicieusement sur de nombreux points. Merci Pierre-François pour ton aide particulièrement précieuse en début de thèse (surtout concernant l'informatique) et pour être un excellent compagnon de pauses café. Partager ton bureau a été très agréable. Merci Christophe pour ton amitié : j'ai beaucoup apprécié nos nombreuses discussions, que ce soit au sujet de la physique que tu expliques si clairement ou au sujet de plein d'autres choses que je n'énumérerai pas ici.

Je remercie également les membres de $B A B A R$ des autres labos français, qui ont contribué à l'excellente ambiance des meetings de collaboration. Merci en particulier à MarieHélène, Marc, Denis, Malcolm, Andreas, José, Vincent et tous les autres! Merci surtout à la bande de "jeunes" de BABAR avec lesquels on a bien fait la fête à Slac et ailleurs : merci à mon grand pote Guillaume qui est toujours de bonne humeur et qui me fait toujours marrer, merci à Fabrice, qui me fait bien marrer aussi, merci à Julie (enfin une fille!) pour toutes nos discussions, merci à Gaël, toujours content et prêt à tout! Je remercie aussi l'ensemble des thésards et post-docs de BABAR qui étaient également compagnons de soirées à Palo Alto ou à San Francisco et en particulier merci aux "anglais", toujours très accueillants dans leur super maison sur Twin Peaks. Merci enfin à Shahram pour son amitié.

Merci à tous les thésards et post-docs du labo qui ont contribué à la bonne ambiance du SPP, en particulier lors des repas-thésards du vendredi, et du gâteau qui suivait généralement. Merci en particulier à Fabrice et Mathieu, mais aussi à Clarisse, Daphné, Jean-Baptiste, Jérémy, Marine, Maximilien, Nicolas, Rachid, Sébastien, Simon, Stéphanie, Vincent... Merci aussi à tous les habitués de la salle café pour l'excellente atmosphère qui y règne. Merci en particulier à Philippe, mon parrain. Merci également aux secrétaires 
du service, Béatrice, Evelyne, Laure et Virginie, pour leur aide et leur patience face à mon incapacité à remplir un papier administratif.

Un grand merci à mes potes de DEA : c'est formidable d'avoir gardé un si bon contact après cette excellente année. Merci surtout à Bruno, Carlos, Delphine, Emmanuel, François, Gilles, Guillaume M, Guillaume T, Johnatan, Martino, Nassim, Thi-Hanh et tous les autres. Merci également à tous mes amis qui m'ont soutenue et encouragée pendant ma thèse : merci bien-sûr à ma grande copine Anne-Gaëlle, mon soutien de toujours, mais aussi à Denis, toujours présent, à Charles, en particulier pour les road trips aux EtatsUnis, à Julien son chalet au ski et tous à toute la bande qui va avec. Merci à Claire, Marie, Paul, Eddy, Sabrina, Charlotte, Thomas, Anne-Sophie, Hélène... Merci aussi à mes amis de Lyon, scientifiques et littéraires.

Je remercie également ma famille pour son soutien et ses encouragements : merci à mes parents, mes frères Romain, Clément et Raphaël, ma petite soeur Blandine. Merci à mes grands-parents, oncles et tantes qui sont venus en masse pour assister à ma soutenance de thèse.

Enfin, je voudrais remercier Romain pour avoir toujours été là pour me motiver et me changer les idées. Merci pour tous les bons moments passés ensemble. 


\section{Table des matières}

Introduction

Chapitre 1 La violation de $C P$ dans le système des mésons $B$

1.1 Les symétries discrètes $C, P$ et $T$. . . . . . . . . . . . . . 13

1.1.1 Importance des symétries en physique . . . . . . . . . . 13

1.1 .2 La parité $P \ldots \ldots \ldots$. . . . . . . . . . . . . . . 13

1.1.3 La conjugaison de charge $C \ldots \ldots$. . . . . . . . . 14

1.1.4 Le renversement du temps $T$. . . . . . . . . . . . . . . . 14

1.1.5 Tests de conservation ou de violation des symétries discrètes $C$, $P$ et $T \ldots \ldots \ldots \ldots \ldots \ldots \ldots \ldots$

1.1.6 La symétrie $C P \ldots \ldots$. . . . . . . . . . . . . . . . . . . . 17

1.1.7 La symétrie CPT . . . . . . . . . . . . . . . . . . . . . 17

1.1.8 Nécessité de la violation de $C P$ pour expliquer l'asymétrie baryonique de l'univers. . . . . . . . . . . . . . . . . . . . . . 18

1.2 Le système des mésons $B$ neutres et la violation de $C P$. . . . . . . . . 19

1.2.1 Formalisme des mésons $B$ neutres . . . . . . . . . . . . . . . . 19

1.2.2 Evolution temporelle des mésons $B$ neutres . . . . . . . . . . 22

1.2.3 Évolution temporelle pour $\Upsilon(4 S) \rightarrow B \bar{B}$ dans le cadre de $B A B A R 23$

1.2.4 Les trois types de violation de $C P \ldots \ldots . \ldots . \ldots 25$ 
1.3 La violation de $C P$ dans le cadre du Modèle Standard . . . . . . . . . 28

1.3.1 Interaction faible et violation de $C P \ldots \ldots . \ldots . . .29$

1.3.2 La matrice CKM . . . . . . . . . . . . . . . . . . . 30

1.3.3 Paramétrisation de Wolfenstein . . . . . . . . . . . . . . . 31

1.3.4 Unitarité de la matrice CKM . . . . . . . . . . . . . . . 31

1.3.5 Connaissances actuelles de la matrice CKM et du triangle d'uni-

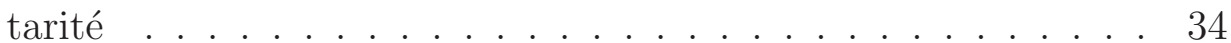

Chapitre 2 Le dispositif expérimental : BABAR et PEP-II 39

2.1 Le collisionneur PEP-II _ . . . . . . . . . . . . . . . . . . . . . . . . . 40

2.2 Le détecteur BABAR . . . . . . . . . . . . . . . . . . . 43

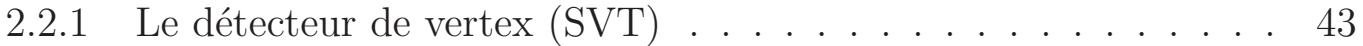

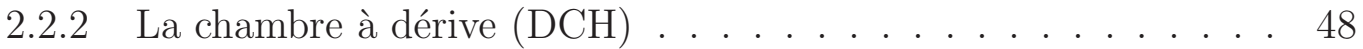

2.2.3 Le DIRC (Detection of Internally Reflected Cherenkov light) . . 52

2.2.4 Le calorimètre électromagnétique . . . . . . . . . . . . . . 56

2.2 .5 Le solenoïde . . . . . . . . . . . . . . . . . . . . . . 61

2.2.6 Le retour de champ instrumenté : IFR (Instrumented Flux Return) 61

2.2.7 Le système de déclenchement . . . . . . . . . . . . . . . . . . . 63

$\begin{array}{ll}\text { Chapitre } 3 \text { L'alignement longitudinal du DIRC } & 67\end{array}$

3.1 Caractérisation de l'alignement du DIRC . . . . . . . . . . . . . . . 68

3.2 Stratégies envisagées pour l'alignement longitudinal du DIRC . . . . . 69

3.3 Alignement par une table de correction . . . . . . . . . . . . . . 70

3.3 .1 Principe . . . . . . . . . . . . . . . . . 70

3.3.2 Procédure pour calculer les corrections . . . . . . . . . . . . . . 72

3.4 Résultats : Amélioration des performances du DIRC . . . . . . . . . 73

3.5 Conclusion . . . . . . . . . . . . . . . . . . . . 74 
Chapitre 4 Comment mesurer $\gamma$ ? $\quad 81$

4.1 Mesure de $\gamma$ avec les modes DK . . . . . . . . . . . . . . . . . . 82

4.1.1 Introduction aux modes $B^{ \pm} \rightarrow D^{0(*)} K^{ \pm(*)} \ldots \ldots$. . . . . . 82

4.1.2 Méthode de Gronau-London-Wyler (GLW) pour les désintégra-

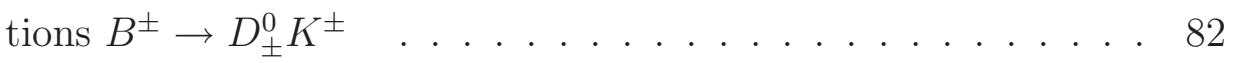

4.1.3 Méthode de Atwood-Dunietz-Soni (ADS) pour les désintégrations $B^{ \pm} \rightarrow D^{0} K^{ \pm} \ldots \ldots \ldots \ldots \ldots$. . . . . . . . 84

4.1.4 Mesure de $\gamma$ dans les désintégrations $B^{ \pm} \rightarrow D^{(*) 0} K^{ \pm}$avec une analyse de Dalitz de la désintégration du $D^{(*) 0} \ldots \ldots$. . . . 85

4.2 Le canal $B \rightarrow D^{* \mp} \pi^{ \pm}$et la mesure de $\sin (2 \beta+\gamma) \ldots \ldots$. . . . . 86

4.2 .1 Introduction . . . . . . . . . . . . . . . . 86

4.2.2 Évolution temporelle de la désintégration $B \rightarrow D^{* \mp} \pi^{ \pm} \ldots 87$

4.2.3 Détermination du rapport $r^{*} \ldots \ldots . \ldots . . \ldots 91$

4.2.4 Valeurs attendues pour les paramètres de $C P \ldots . . . .92$

$\begin{array}{ll}\text { Chapitre } 5 \text { Analyse } & 93\end{array}$

5.1 La méthode de reconstruction partielle . . . . . . . . . . . . . . 94

5.1.1 Principe de la méthode de reconstruction partielle . . . . . . . . 94

5.1 .2 La masse manquante $: m_{\text {rec }} \ldots \ldots . \ldots . \ldots 97$

5.1 .3 Le discriminant de Fisher $F \ldots \ldots$. . . . . . . . . . . . . 98

5.1 .4 Étiquetage de la saveur du $B \ldots \ldots 101$

5.1.5 Mesure de la distance entre les vertex des deux $B$. . . . . . 102

5.2 Événements utilisés . . . . . . . . . . . . . . . . . 106

5.2.1 Présélection au niveau de la production . . . . . . . . . 106

5.2.2 Sélection du meilleur candidat . . . . . . . . . . . . . . 109

5.2.3 Échantillons de données utilisées . . . . . . . . . . . . . 109

5.3 Procédure de l'analyse . . . . . . . . . . . . . . . . . . . . . . . . . 109

5.4 Ajustement des variables cinématiques . . . . . . . . . . . . . . . 111

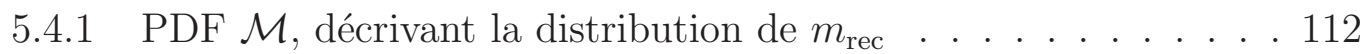


5.4.2 PDF $\mathcal{F}$, décrivant la distribution de $F$. . . . . . . . . . . . . 112

5.4.3 Ajustement total des variables cinématiques . . . . . . . . . . . 114

5.4.4 Résultats de l'ajustement des variables cinématiques . . . . . . 115

5.4.5 Détermination des paramètres $\alpha$ et $\rho \ldots$. . . . . . 116

5.4.6 Résultats de l'ajustement de $\alpha$ et $\rho$. . . . . . . . . . . 120

5.5 Analyse en $\Delta t$ pour le signal $D^{*} \pi \ldots \ldots \ldots$. . . . . . . . . . . . . . . . 121

5.5.1 Introduction sur l'analyse en $\Delta t \ldots \ldots \ldots$. . . . . . . . 21

5.5.2 PDF en $\Delta t$ du signal $D^{*} \pi$ pour les événements étiquetés par une trace issue de $B_{\text {tag }} \ldots \ldots \ldots$. . . . . . . . . . 121

5.5.3 Effet des leptons de cascade sur la paramétrisation de la PDF . 125

5.5.4 Effet de la reconstruction partielle sur la paramétrisation de la PDF en $\Delta t \ldots \ldots \ldots \ldots \ldots$

5.5.5 Validation sur Monte-Carlo pour les événements étiquetés par un lepton . . . . . . . . . . . . . . . . . . . 132

5.5.6 Validation sur Monte-Carlo pour les événements étiquetés par un kaon . . . . . . . . . . . . . . . . . . . . 142

5.5.7 Conclusions de la validation sur Monte-Carlo . . . . . . . . . 143

5.6 Analyse en $\Delta t$ des bruits de fond . . . . . . . . . . . . . . . . 144

5.6 .1 PDFs des bruits de fond . . . . . . . . . . . . . . . 144

5.6.2 Validation sur Monte-Carlo du traitement des bruits de fond pour les événements étiquetés par un lepton . . . . . . . . 146

5.6.3 Validation sur Monte-Carlo du traitement des bruits de fond pour les événements étiquetés par un kaon . . . . . . . . . . . 148

5.6.4 Conclusion de l'étude sur Monte-Carlo . . . . . . . . . . . . . . 149

5.7 Résultats de l'analyse . . . . . . . . . . . . . . . . 150

5.7.1 Résultats de l'analyse en $\Delta t$ pour les événements étiquetés par un lepton . . . . . . . . . . . . . . . . 150

5.7.2 Validation de l'ajustement avec des expériences de Monte-Carlo 159

5.7.3 Résultats de l'analyse en $\Delta t$ pour les événements étiquetés par un kaon . . . . . . . . . . . . . . . . . 162

5.8 Étude des erreurs systématiques . . . . . . . . . . . . . . . 166

5.8.1 Systématiques dues à la méthode de l'ajustement en quatre étapes166

5.8.2 Systématiques dues au dispositif expérimental . . . . . . . . . 167 
5.8.3 Systématiques dues à l'éventuelle présence de violation de $C P$ dans le bruit de fond . . . . . . . . . . . . . . . . 168

5.8.4 Autres sources d'erreurs systématiques . . . . . . . . . . . 170

5.8.5 Résumé des erreurs systématiques . . . . . . . . . . . . . . . 171

5.9 Combinaison des résultats . . . . . . . . . . . . . . . . . . 171

Chapitre 6 Interprétation des résultats $\quad 173$

6.1 Intervalles de confiance classiques . . . . . . . . . . . . . . . . . . 173

6.1 .1 Principe de la méthode de Neyman . . . . . . . . . . . . . . 173

6.1.2 Cas gaussien non borné . . . . . . . . . . . . . . . . 174

6.1.3 Cas gaussien borné . . . . . . . . . . . . . . . . 177

6.2 Méthode de Feldman-Cousins . . . . . . . . . . . . . . . 178

6.2.1 Distributions poissonniennes . . . . . . . . . . . 178

6.2.2 Distributions gaussiennes bornées . . . . . . . . . . . . 179

6.2.3 Définition du $\Delta \chi^{2} \ldots \ldots \ldots$. . . . . . . . . . . . . . . . . 179

6.3 Application au cas de la mesure de $\sin (2 \beta+\gamma) \quad \ldots . . . .180$

6.3.1 Méthode avec une hypothèse sur $S U(3) \quad \ldots . .180$

6.3.2 Méthode avec un scan de $r^{*} \ldots$. . . . . . . . . . . 187

6.4 Combinaisons des différentes mesures de $\sin (2 \beta+\gamma)$ par BABAR . . . . 188

6.5 Combinaison mondiale de toutes les mesures de $\sin (2 \beta+\gamma) \quad$. . . . . 190

$\begin{array}{ll}\text { Conclusion } & 193\end{array}$

$\begin{array}{ll}\text { Bibliographie } & 195\end{array}$ 
Table des matières 


\section{Introduction}

Ce mémoire présente les travaux réalisés pendant ma thèse au Service de Physique des Particules du CEA-Saclay dans l'expérience BABAR. La partie principale consiste en un travail d'analyse des données afin d'étudier la violation de $C P$ dans les désintégrations $B \rightarrow D^{* \mp} \pi^{ \pm}$partiellement reconstruites, avec le détecteur BABAR. Une autre partie concerne une étude portant sur l'alignement du détecteur d'identification de particules par effet Cherenkov : le DIRC.

L'étude de la violation de $C P$ demeure l'une des composantes fondamentales de la physique des particules. L'existence de la violation de $C P$ est l'une des trois conditions nécessaires à la prépondérance de la matière par rapport à l'anti-matière dans l'univers, ainsi que l'avait énoncé Sakharov en 1967. Une bonne compréhension de ce phénomène nous aiderait ainsi à mieux comprendre la formation de l'univers. De plus, la violation de $C P$ est prédite dans le cadre du Modèle Standard. Elle a été observée pour la première fois en 1964 dans le système des mésons K. Le principal but de l'expérience BABAR est d'étudier de façon systématique et complète la violation de $C P$ dans le système des mésons $B$, en particulier par la mesure des angles $\alpha, \beta$ et $\gamma$ et des côtés du triangle d'unitarité. Le détecteur BABAR est situé auprès de l'accélérateur PEP-II usine à $B$ qui produit environ 100 millions de $B^{0}$ en un an. Les $B$ étant produits en très grande quantité, il est possible d'étudier les désintégrations rares des $B$ et de mesurer les paramètres de $C P$ dans de nombreux modes différents. On dispose ainsi de nombreuses mesures redondantes, qui permettent de tester le Modèle Standard et sa cohérence. La violation de $C P$ a été mise en évidence dès 2001 dans le système des mésons $B$ par la mesure de $\sin (2 \beta)$ dans les désintégrations $B^{0} \rightarrow J / \Psi K_{S}$. Cela prouve que la violation de $C P$ n'est pas un phénomène propre aux kaons et valide la description de la violation de $C P$ par la matrice CKM dans le cadre du Modèle Standard. Nous sommes actuellement dans une seconde phase, où l'on essaie de surcontraindre le triangle d'unitarité, en particulier par la mesure des deux autres angles $\alpha$ et $\gamma$.

Les désintégrations $B \rightarrow D^{* \mp} \pi^{ \pm}$permettent la mesure de $\sin (2 \beta+\gamma)$ par une analyse de violation de $C P$ dépendante du temps. $B^{0}$ peut en effet se désintégrer en $D^{*-} \pi^{+}$directement ou après s'être mélangé en $\bar{B}^{0}$. On peut donc envisager une effet de violation de $C P$ dans l'interférence entre le mélange et la désintégration. La phase faible $\gamma$ provient de l'interférence entre les transitions $(b \rightarrow c)$ et $(b \rightarrow u)$ et la phase faible $2 \beta$ du mélange $B^{0} \bar{B}^{0}$. La principale difficulté de la mesure réside dans le fait que les asymétries attendues sont très petites. Il est donc nécessaire d'avoir un maximum de statistique pour étudier la violation de $C P$ dans ce mode. La désintégration $B \rightarrow D^{* \mp} \pi^{ \pm}$est suivie de 
la désintégration $D^{* \mp} \rightarrow D^{0} \pi_{s}^{\mp}$. Afin de sélectionner le maximum d'événements possible, on utilise une technique de reconstruction partielle du $B^{0}$ qui se désintègre en $D^{*} \pi$. Cela consiste à ne reconstruire que les deux pions issus des désintégrations du $B$ et du $D^{*}$, sans chercher à reconstruire le $D^{0}$. Cette méthode permet d'augmenter la statistique d'un ordre de grandeur, mais on a en contrepartie beaucoup de bruit fond. Une autre difficulté de l'analyse est due au fait que les traces du $D^{0}$ non reconstruit peuvent être utilisées à tort et biaiser ainsi l'analyse. L'analyse présentée ici a conduit à la mesure d'asymétries de $C P$. Les résultats ont été utilisés afin de déterminer un niveau de confiance en fonction de $\sin (2 \beta+\gamma)$, grâce à une méthode d'interprétation fréquentiste. On peut alors en déduire une limite sur la valeur de $\sin (2 \beta+\gamma)$, ainsi que des contraintes dans le plan $(\rho, \eta)$.

L'autre volet de mon travail de thèse porte sur l'alignement du DIRC, détecteur d'identification des particules par effet Cherenkov. Ce détecteur est primordial pour discriminer les pions des kaons jusqu'à $4 \sigma$ pour une impulsion de $3 \mathrm{GeV} / \mathrm{c}$. Le principe consiste à mesurer l'angle d'émission des photons de Cherenkov par rapport à la direction de la particule chargée qui traverse le milieu radiateur du DIRC. La résolution sur la mesure de cet angle est principalement limitée par un mauvais alignement du DIRC. 
Contexte théorique 



\section{1 \\ La violation de $C P$ dans le système des mésons $B$}

\section{Introduction}

Après quelques rappels sur les symétries $C, P$ et $T$, ce chapitre expose la théorie des systèmes de mésons $B$ et présente les différents types de violations de $C P$ que l'on peut observer avec les $B$. On se place ensuite dans le cadre du Modèle Standard pour introduire la matrice CKM et montrer qu'il est possible de contraindre les paramètres de cette matrice grâce à l'étude de l'asymétrie $C P$ dans le systèmes des mésons $B$.

\subsection{Les symétries discrètes $C, P$ et $T$}

\subsubsection{Importance des symétries en physique}

On définit une symétrie comme étant une transformation qui laisse invariante une loi de la physique. En théorie des champs, cela se traduit par l'invariance du Lagrangien et des équations de mouvement associées sous un changement de variable. Le concept de symétrie est fondamental en physique et permet en particulier de simplifier une théorie. Cela permet par exemple d'établir des lois de conservation. Il existe diverses sortes de symétries : locales (qui dépendent des coordonnées) ou globales, continues ou discrètes. Emmy Noether avait stipulé dès 1918 qu'à toute famille continue de symétries d'un système physique est associée une loi de conservation pour ce système [1]. Par exemple, la conservation de l'énergie et de l'impulsion découle directement de l'invariance par translation du temps et de l'espace. Il existe des symétries d'un autre type, qui sont globales et discrètes, et auxquelles nous allons nous intéresser ici. Il s'agit des symétries $C$ (conjugaison de charge), $P$ (parité) et $T$ (renversement du temps).

\subsubsection{La parité $P$}

La parité consiste à inverser les coordonnées spatiales de l'espace à trois dimensions. Cela revient à changer le vecteur position $\vec{r}$ en $-\vec{r}$ et par conséquent le vecteur impulsion 
$\vec{p}=m d \vec{r} / d t$ devient $-\vec{p}$. La conséquence immédiate est que le moment orbital $\vec{L}=$ $\vec{r} \times \vec{p}$ est conservé. Il en est de même pour le moment cinétique. Cette transformation est décrite par un opérateur unitaire et hermitien qui a donc pour valeurs propres \pm 1 . Cet opérateur agit de la façon suivante sur un état $f$ d'impulsion $\vec{p}$ et de spin $s$ :

$$
P|f(\vec{p}, s)\rangle=\eta_{P}|f(-\vec{p}, s)\rangle .
$$

On définit ainsi la parité intrinsèque d'une particule par un nombre quantique $\eta_{P}$ égal à \pm 1 . Par convention, on a défini la parité des quarks et des leptons comme étant égale à 1 . On peut démontrer dans le cadre des équations d'onde relativiste que dans le cas des fermions (particules de spin demi-entier), une particule et son anti-particule ont des parités opposées alors que dans le cas des bosons (particules de spin entier), une particule et son anti-particule ont des parités égales. Considérons un système de deux particules notées $a$ et $b$. La parité de ce système est $\eta=\eta_{a} \eta_{b}(-1)^{l}$, où $\eta_{a}$ et $\eta_{b}$ sont les parités des particules $a$ et $b$ respectivement et $l$ représente la valeur propre du moment cinétique du mouvement relatif. On en déduit qu'avec les conventions exposées précédemment, la parité d'un système fermion/anti-fermion est $\eta=(-1)^{l+1}$. La parité est conservée par les interactions électromagnétique et forte, mais violée de façon maximale par l'interaction faible.

\subsubsection{La conjugaison de charge $C$}

La conjugaison de charge est une transformation qui consiste à remplacer une particule par son anti-particule. La notion d'anti-particule a été introduite par Dirac en 1928 pour l'électron et a été ensuite étendue à toutes les particules. On associe ainsi à une particule une anti-particule de même masse et spin, mais de charges (électrique, baryonique, leptonique, etc) opposées. La conjugaison de charge est également décrite par un opérateur unitaire hermitien qui transforme une particule en une anti-particule de la façon suivante, sans modifier l'impulsion ou le spin de la particule :

$$
C|f(\vec{p}, s)\rangle=\eta_{C}|\bar{f}(\vec{p}, s)\rangle,
$$

avec $\eta_{C}= \pm 1$. De même que la parité, la conjugaison de charge est conservée par les interactions électromagnétique et forte, mais violée par l'interaction faible. La conservation de $C$ par les interactions électromagnétique et forte implique des règles de sélections sur les réactions entre les particules.

\subsubsection{Le renversement du temps $T$}

Le renversement du temps revient à transformer la coordonnée temporelle $t$ en $-t$. Ainsi, cela ne modifie le vecteur $\vec{r}$, mais change le signe du vecteur vitesse $\vec{v}$, et donc de l'impulsion $\vec{p}$ et des moments cinétiques en général, que ce soit le moment orbital au sens classique du terme ou le spin de la particule, qui est le moment orbital intrinsèque de la particule. L'opérateur $T$ agit donc ainsi :

$$
T|f(\vec{p}, s)\rangle=\eta_{T}|\bar{f}(-\vec{p},-s)\rangle^{*}
$$


où * signifie qu'il s'agit de la conjugaison complexe.

Notons que contrairement aux opérateurs $C$ et $P$ qui sont unitaires, l'opérateur $T$ est anti-unitaire.

\subsubsection{Tests de conservation ou de violation des symétries dis- crètes $C, P$ et $T$}

Les symétries $C, P$ et $T$ sont conservées par les interactions électromagnétique et forte, mais violées par l'interaction faible. De nombreuses expériences ont permis de tester la conservation ou la violation de ces symétries par les différentes interactions.

\section{La parité}

Jusque dans le milieu des années 1950, la conservation de la parité n'avait jamais été remise en cause. En 1956, Lee et Yang [2] émettent l'hypothèse que la parité peut être violée par l'interaction faible et proposent une série d'expériences permettant de tester cela. Madame $\mathrm{Wu}$ apporte alors la preuve expérimentale de la violation de $P$ par l'interaction faible en étudiant la désintégration $\beta \mathrm{du}{ }^{60} C o$ polarisé [3]. Cette expérience montre que les électrons sont préférentiellement émis dans la direction opposée au spin, ce qui est interdit si la parité est conservée. De plus, la violation de parité par l'interaction faible permet d'expliquer l'énigme des particules $\theta$ et $\tau$. En effet on avait observé les désintégrations $\theta^{+} \rightarrow \pi^{+} \pi^{0}$ et $\tau^{+} \rightarrow \pi^{+} \pi^{-} \pi^{+}$. Les états finals de ces deux désintégrations ont des parités opposées. C'est pourquoi on pensait que la particule mère n'était pas la même dans les deux cas, bien que les masses de $\theta$ et $\tau$ soient très proches l'une de l'autre. Il s'agissait en réalité d'une même particule (le méson $K^{+}$), dont la désintégration faible viole la parité. Plusieurs expériences sont alors mises au point afin de tester la conservation de la parité par l'interaction forte, comme par exemple l'expérience de Wilkinson en 1958 [4] qui consiste à étudier la désintégration forte : ${ }^{6} \mathrm{Li}^{*} \rightarrow^{4} \mathrm{He}+d$. Cette désintégration est énergétiquement permise mais viole la parité. En effet les valeurs de spin-parité du ${ }^{6} \mathrm{Li}^{*}$, de $\mathrm{l}^{4} \mathrm{He}$ et $\mathrm{du} d$ sont respectivement $0^{+}, 0^{+}$et $1^{+}$. La conservation du moment angulaire impose que l'état final ait un moment angulaire égal à 1 , impliquant dans ce cas-là que la parité soit $\eta_{P}=-1$, ce qui n'est pas compatible avec la parité de l'état initial. Il a été montré que cette réaction est exclue avec une sensibilité de $10^{-7}$, et donc que la parité n'est pas violée par l'interaction forte dans cette désintégration.

\section{La conjugaison de charge}

Une façon de tester la conservation de la conjugaison de charge consiste à comparer une réaction avec la réaction conjuguée de charge dans laquelle on remplace les particules par leur anti-particule. Si $C$ est conservée, les deux réactions, images l'une de l'autre par conjugaison de charge, ont les mêmes propriétés. On a par exemple mesuré les sections efficaces de production de $\pi^{+}$et de $\pi^{-}$par l'annihilation d'une paire de proton/antiproton $p \bar{p}$. Elles sont égales à mieux de $1 \%$ [5], ce qui démontre la conservation de $C$ par l'interaction forte. La conservation de $C$ par l'interaction électromagnétique a été testée en étudiant la désintégration du méson $\eta\left(\eta_{C}(\eta)=1\right)$. En particulier, on a cherché 
à mesurer le rapport d'embranchement de la désintégration $\eta \rightarrow \pi^{0} \mu^{+} \mu^{-}$, qui viole $C$. Cette désintégration n'a pas été observée expérimentalement [6]. Le fait que l'univers soit uniquement composé de matière nécessite que $C$ soit violée, comme on le vera dans la suite (voir partie 1.1.8). Cet argument cosmologique est une très forte incitation à chercher à expliquer la violation de $C P$ par l'une des interactions fondamentales. L'interaction faible est un bon candidat pour cela. L'étude de la désintégration leptonique du $\pi^{ \pm}$a permis de mettre en évidence la violation de $C$ par l'interaction faible et du même coup la violation de $P$. La désintégration $\pi^{ \pm} \rightarrow l^{ \pm} \nu_{l}$ est due à l'interaction faible, car la durée de vie du $\pi^{ \pm}$ est trop longue $\left(\sim 2.610^{-8} s\right)$ pour être due à une autre interaction. De plus il ne peut y avoir de production de neutrino que par l'interaction faible. Le spin du pion est 0, alors que le spin des deux leptons produits est 1/2. Il existe donc a priori deux possibilités de désintégration (figure 1.1) : la polarisation du neutrino peut être droite (le spin est dans la même direction que l'impulsion) ou gauche (le spin et l'impulsion ont des directions opposées).

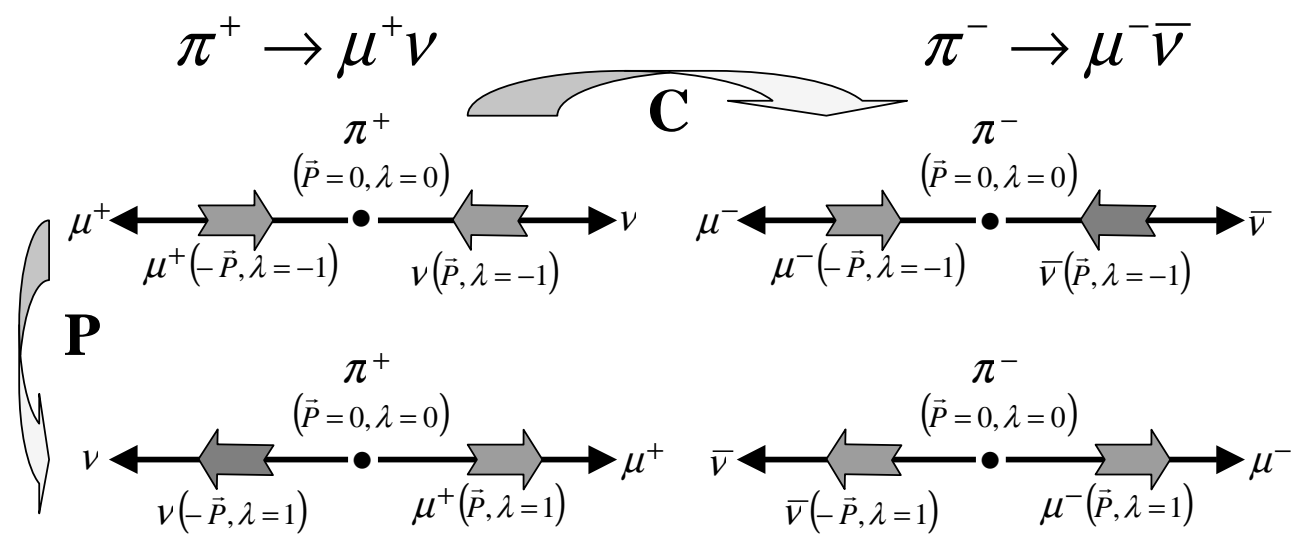

FIG. 1.1: Désintégration du $\pi^{ \pm}$en muon et neutrino muonique.

Les désintégrations de la première et de la deuxième ligne sont reliées par la symétrie $P$ alors que les désintégrations de la première et de la deuxième colonne sont reliées par la symétrie $C$. Or seules la première et la dernière désintégrations sont observées expérimentalement. De manière plus générale, on n'a jamais observé de neutrino droit ou d'anti-neutrino gauche dans la nature. Cette expérience démontre donc la non conservation de la parité et de la conjugaison de charge par l'interaction faible. En revanche, si les deux symétries sont appliquées, la symétrie $C P$ semble être conservée.

\section{Le renversement du temps}

Il est possible de tester l'invariance des interactions fondamentales par renversement du temps en étudiant l'amplitude de transition d'un état $a$ vers un état $b$ et réciproquement. Si 
T est conservée, ces amplitudes doivent être égales. Par exemple, Barshay [7] et Sober [8] ont étudié les désintégrations réciproques $\gamma+d \rightleftarrows n+p$ à la fin des années soixante. Les résultats sont compatibles avec la conservation de $T$. Jusqu'à présent, la violation de $T$ n'a pas été observée pour l'interaction forte et électromagnétique. Elle a en revanche été observée pour l'interaction faible par l'expérience CPLEAR [9] par l'intermédiaire des désintégrations semi-leptoniques $K^{0} \rightarrow \pi e \nu$.

\subsubsection{La symétrie $C P$}

La désintégration leptonique des pions chargés a montré que la parité et la conjugaison de charge étaient violées de façon maximale par l'interaction faible. En revanche, la symétrie $C P$ est conservée dans cette désintégration. La première observation de la violation de $C P$ a été réalisée par Christenson, Cronin, Fitch et Turlay en 1964 [10], en étudiant les mésons $K$ neutres. Il existe deux états propres physiques pour les $K$ neutres : le $K_{L}$, caractérisé par sa longue durée de vie et le $K_{S}$, caractérisé par sa plus courte durée de vie. Le $K_{L}$ se désintègre principalement en $\pi^{0} \pi^{+} \pi^{-}$, état propre de $C P-1$. Ils ont cependant observé la désintégration du $K_{L}$, que l'on croyait être état propre de $C P$-1, en $\pi^{+} \pi^{-}$, état propre de $C P$ de valeur propre +1 . Cette désintégration est la signature de la violation de $C P$ par l'interaction faible dans le système des mésons $K$. La découverte de la violation de $C P$ a entraîné des recherches très poussées dans le secteur des kaons. Plus récemment, on a cherché à savoir si la violation de $C P$ était un phénomène spécifique aux kaons, ou si elle pouvait intervenir dans d'autres mésons. C'est cette motivation qui a conduit à construire des expériences telles que $B A B A R$, dont le principal but est l'étude de la violation de $C P$ dans les mésons $B$. L'étude de la violation de $C P$ dans le cadre des $B$ est très prometteuse dans le sens où les contraintes attendues sur les paramètres du modèles sont fortes.

La violation de $C P$ directe a été observée dans le secteur des K en 1999 [11,12]. Cette mesure est un accord avec le Modèle Standard et a permis d'exclure le modèle super-faible. Elle a également été observée en 2004 dans le secteur des $B$ par les expériences BABAR [13] et Belle [14].

\subsubsection{La symétrie $C P T$}

La symétrie CPT est conservée en théorie des champs $[15,16]$ dès lors que l'on requiert la localité, l'invariance de Lorentz et l'hermiticité du Lagrangien, ce qui est le cas de la plupart des théories utilisées. Une conséquence de la conservation de CPT est qu'une particule et une anti-particule ont la même masse et le même temps de vie. On peut ainsi tester $C P T$ en mesurant par exemple la différence de masse entre un électron et un positron ou entre un proton et un anti-proton. Pour l'instant, on n'a pas mis en évidence expérimentalement la violation de $C P T$. 


\subsubsection{Nécessité de la violation de $C P$ pour expliquer l'asymétrie baryonique de l'univers.}

L'univers dans lequel nous vivons est essentiellement constitué de matière. A l'échelle du système solaire, on n'observe que de la matière. On a cependant détecté de l'antimatière à l'échelle extra-solaire dans les rayons cosmiques, mais en quantité très faible par rapport à la matière.

Pourtant, au tout début de l'histoire de l'univers, on pense qu'il y avait autant de baryons que d'anti-baryons, ce qui veut dire en d'autres termes qu'il y avait autant de matière que d'anti-matière. Il existait alors un équilibre thermique tel que les nombres de baryons, anti-baryons et photons étaient quasiment les mêmes : $n_{b} \sim n_{\bar{b}} \sim n_{\gamma}$. Lors du refroidissement de l'univers dû à son expansion, cet équilibre thermique a été rompu et les baryons et les anti-baryons se sont annihilés pour former des photons. C'est pourquoi il y a maintenant beaucoup plus de photons que de baryons dans l'univers $\left(n_{b} \sim 10^{-10} n_{\gamma}\right)$. On observe également que le nombre de baryons est très grand devant le nombre d'anti-baryons. Cela n'est possible que s'il y a eu un excès de baryons par rapport aux anti-baryons, car l'annihilation en photons fait disparaître autant de baryons que d'anti-baryons.

Sakharov a émis l'hypothèse en 1967 [17] qu'il était possible pour un univers initialement symétrique de créer un excès de baryons par rapport aux anti-baryons de l'ordre de $10^{-10}$. Pour cela, trois conditions sont nécessaires :

- la violation du nombre baryonique

- la violation de la symétrie $C$ et de la symétrie $C P$

- la rupture de l'équilibre thermique de l'univers

La nécessité de violer le nombre baryonique $B=\left(n_{q}-n_{\bar{q}}\right) / 3$, où $n_{q}\left(n_{\bar{q}}\right)$ représente le nombre de quarks (anti-quarks), est évidente si on veut créer un excès de baryons à partir d'un univers symétrique. Dans le cadre du Modèle Standard, la violation du nombre baryonique n'est possible que par des processus non perturbatifs. Cependant, ces phénomènes sont très supprimés, car ils nécessitent de vaincre une barrière de potentiel énorme. Au moment de l'univers primordial, l'énergie thermique très importante a pu permettre de franchir cette barrière. La violation du nombre baryonique est également possible dans le cadre des théories de grande unification. Pour qu'il y ait violation du nombre baryonique, il faut qu'il existe des bosons de jauge massifs et des Higgs. La création du léger excès de matière par rapport à l'anti-matière n'a donc pu se faire qu'après la brisure de symétrie de grande unification. Selon la théorie de grande unification, couplée à la supersymétrie, cette création de l'excès d'anti-matière se serait produite à une échelle d'énergie $\mathrm{T}<10^{16} \mathrm{GeV}$. La théorie de grande unification, basée sur le groupe $S U(5)$, sans supersymétrie, est défavorisée par des mesures expérimentales telles que la durée de vie du proton ou la variation des constantes de couplage.

La nécessité de la violation de $C$ découle directement liée à la violation du nombre baryonique. En effet, si on applique la transformation $C$ à un système, on change le signe de son nombre baryonique $B$. Le nombre baryonique de l'univers est défini ainsi : $B=\left(n_{q}-n_{\bar{q}}\right) / s$ où $s$ désigne la densité d'entropie. Ainsi, si $C$ n'était pas violée, l'univers qui avait initialement un nombre baryonique nul aurait conservé ce nombre baryonique nul, 
empêchant ainsi la formation d'un excès de baryons. De plus, comme l'univers, supposé homogène et isotrope, n'a pas de direction privilégiée, il est également nécessaire que $C P$ soit violée.

La dernière condition à remplir (la rupture de l'équilibre thermique de l'univers) est nécessaire, car la conservation de CPT par les interactions fondamentales impose qu'il ne peut y avoir de différence de densité entre la matière et l'anti-matière dans un univers en équilibre thermique.

Les trois conditions de Sakharov, nécessaires à l'asymétrie matière/anti-matière, ont été réunies au moment de la brisure de la symétrie de grande unification. L'argument cosmologique incite fortement les physiciens à étudier la violation de $C P$ en détails. Toutefois, la violation de $C P$ prédite par le Modèle Standard ne permet pas d'expliquer l'asymétrie observée dans l'univers. De plus, les expériences que l'on est capable de construire à l'heure actuelle en physique des particules correspondent à un univers "froid", alors que le phénomène décrit par Sakharov se déroule dans un univers "chaud", avant l'annihilation baryonique. Ainsi, les phénomènes de violation de $C P$ que l'on étudie dans les expériences actuelles ne sont pas forcément de même type que celui qui a eu lieu au moment de la rupture de la symétrie matière/anti-matière de l'univers. Néanmoins, une bonne compréhension de la violation de $C P$ dans le cadre du Modèle Standard peut permettre de comprendre des phénomènes analogues qui auraient permis cette asymétrie. De plus, l'étude de la violation de $C P$ permet un excellent test du Modèle Standard et éventuellement de ses extensions, dans un domaine qui encore récemment n'était que peu connu.

\subsection{Le système des mésons $B$ neutres et la violation de $C P$}

\subsubsection{Formalisme des mésons $B$ neutres}

\section{Introduction}

On distingue deux types de mésons $B$ neutres : les mésons $B_{d}$, constitués d'un antiquark $\bar{b}$ et d'un quark $d$ et les mésons $B_{s}$, constitués d'un anti-quark $\bar{b}$ et d'un quark $s$. Dans l'expérience $B A B A R$, on ne produit pas de mésons $B_{s}$. Dans la suite, on ne s'intéressera donc qu'aux mésons $B_{d}$ et on s'affranchira de l'indice ${ }_{d}$. Les états propres de saveur des mésons $B_{q}$ sont $B^{0}=\bar{b} q$ et $\bar{B}^{0}=b \bar{q}$. Le fait d'utiliser les états propres de saveur pour les mésons $B$ neutres est pratique, car leur contenu en quark est bien défini et donc il est facile de comprendre les phénomènes de productions de particules et de désintégrations. Cependant, ces états propres de saveur ne sont pas les états propres physiques (également appelés états propres de masse). Les états propres physiques sont caractérisés par une masse et un temps de vie bien définis. On les appelle $B_{H}(\mathrm{H}$ pour "heavy", signifiant lourd) et $B_{L}$ (L pour "light", signifiant léger). Notons que c'est l'interaction faible qui est à l'origine de cette différence entre les états propres de masse et les états propres de saveur, car elle permet la transition d'un quark à l'autre. Le $B^{0}$ et le $\bar{B}^{0}$ peuvent donc osciller l'un vers l'autre. C'est ce que l'on appelle le phénomène de mélange : le $B^{0}$ et le $\bar{B}^{0}$ oscillent à la fréquence $\Delta m$, qui est égale à la différence de masse entre les deux états 
propres physiques.

\section{Équation de Schrödinger et caractérisation du Hamiltonien}

On cherche à déterminer les états physiques, qui sont états propres du Hamiltonien par définition, en fonction des états de saveur, sachant que $B_{H}$ et $B_{L}$ sont des combinaisons linéaires de $B^{0}$ et $\bar{B}^{0}$.

Considérons une combinaison linéaire quelconque de $B^{0}$ et $\bar{B}^{0}$ :

$$
a\left|B^{0}\right\rangle+b\left|\bar{B}^{0}\right\rangle
$$

Cet état doit satisfaire l'équation de Schrödinger, dépendant du temps :

$$
i \frac{d}{d t}\left(\begin{array}{c}
a \\
b
\end{array}\right)=\mathcal{H}\left(\begin{array}{c}
a \\
b
\end{array}\right) \equiv\left(M-\frac{i}{2} \Gamma\right)\left(\begin{array}{c}
a \\
b
\end{array}\right) .
$$

L'Hamiltonien $\mathcal{H}$ est composé de deux matrices hermitiennes $M$ et $\Gamma$ qui ont des significations physiques bien définies [18] :

- $M$, appelée matrice de masse, est la composante dispersive, due aux processus mettant en jeu des états intermédiaires virtuels :

- $M_{11}$ et $M_{22}$ correspondent aux masses des quarks constituant les mésons $B_{d}$ et à leur énergie de liaison,

- $M_{12}$ et $M_{21}$ sont générés par les transitions $B^{0} \leftrightarrows \bar{B}^{0}$ avec des processus virtuels.

- $\Gamma$, appelée matrice des désintégrations est la composante absorptive, due aux processus mettant en jeu des états intermédiaires réels :

- $\Gamma_{11}$ et $\Gamma_{22}$ correspondent aux transitions $B^{0} \rightarrow f$ et $\bar{B}^{0} \rightarrow \bar{f}$,

- $\Gamma_{12}$ et $\Gamma_{21}$ correspondent aux transitions $B^{0} \rightarrow f \rightarrow \bar{B}^{0}$ et $\bar{B}^{0} \rightarrow f \rightarrow B^{0}$, où $f$ est un état final de désintégration commun à $B^{0}$ et $\bar{B}^{0}$.

Il existe certaines relations entre les paramètres de la matrice $\mathcal{H}$. La relation générale qui relie $\left|B^{0}\right\rangle$ et $\left|\bar{B}^{0}\right\rangle$ sous l'action de $C P$ est :

$$
C P\left|B^{0}\right\rangle=e^{i \phi}\left|\bar{B}^{0}\right\rangle
$$

où $\phi$ est une phase arbitraire, dont on peut fixer la valeur une fois pour toutes. Afin de simplifier les calculs, on choisit $\phi=0^{1}$ et on a donc la relation :

$$
C P\left|B^{0}\right\rangle=\left|\bar{B}^{0}\right\rangle
$$

Le choix de phase implique également :

$$
T\left|B^{0}\right\rangle=\left|B^{0}\right\rangle^{*}
$$

\footnotetext{
${ }^{1}$ On peut se référer au cours de T. Nakada [19] qui traite tout le formalisme sans fixer la valeur de cette phase.
} 
L'invariance de $\mathcal{H}$ sous la symétrie $C P T$ se traduit de la façon suivante :

$$
(C P T)^{\dagger} \mathcal{H}(C P T)=\mathcal{H}
$$

En combinant les équations $1.7,1.8$ et 1.9 , on trouve :

$$
\left\langle B^{0}|\mathcal{H}| B^{0}\right\rangle=\left\langle\bar{B}^{0}|\mathcal{H}| \bar{B}^{0}\right\rangle
$$

On en déduit que la conservation de $C P T$ impose $H_{11}=H_{22}$.

On peut également montrer que si $T$ est conservée alors $\left|H_{12}\right|=\left|H_{21}\right|$. Comme les matrices $M$ et $\Gamma$ sont hermitiennes, on a $M_{12}=M_{21}^{*}$ et $\Gamma_{12}=\Gamma_{21}^{*}$ et la condition $\left|H_{12}\right|=$ $\left|H_{21}\right|$ signifie donc que la phase relative entre $M_{12}$ et $\Gamma_{12}$ est égale à 0 ou $\pi$.

L'invariance par $C P$ implique à la fois que la phase relative entre $M_{12}$ et $\Gamma_{12}$ soit égale à 0 ou $\pi$ et que $H_{11}=H_{22}$. Ainsi, la violation de la symétrie $C P$ est établie si la symétrie $T$ est violée ou si la symétrie $C P T$ est violée.

\section{Détermination des états physiques en fonction des états propres de saveur}

Pour trouver les états propres physiques $B_{H}$ et $B_{L}$, dont les valeurs propres sont $\lambda_{H}$ et $\lambda_{L}$, il faut diagonaliser $\mathcal{H}$. Comme ce sont des états propres physiques, ils ont une masse et une largeur bien définies que l'on note pour $B_{H}\left(B_{L}\right): M_{H}\left(M_{L}\right)$ et $\Gamma_{H}\left(\Gamma_{L}\right)$. Ces paramètres sont reliés aux valeurs propres :

$$
\begin{aligned}
& \lambda_{H}=H_{11}+\sqrt{H_{12} H_{12}^{*}}=M_{H}-i \frac{\Gamma_{H}}{2}, \\
& \lambda_{L}=H_{11}-\sqrt{H_{12} H_{12}^{*}}=M_{L}-i \frac{\Gamma_{L}}{2} .
\end{aligned}
$$

On en déduit donc :

$$
\begin{aligned}
& M_{H}=M_{11}+R e \sqrt{\left(M_{12}-\frac{i}{2} \Gamma_{12}\right)\left(M_{12}^{*}-\frac{i}{2} \Gamma_{12}^{*}\right)}, \\
& \Gamma_{H}=\Gamma_{11}-2 I m \sqrt{\left(M_{12}-\frac{i}{2} \Gamma_{12}\right)\left(M_{12}^{*}-\frac{i}{2} \Gamma_{12}^{*}\right)}, \\
& M_{L}=M_{11}-R e \sqrt{\left(M_{12}-\frac{i}{2} \Gamma_{12}\right)\left(M_{12}^{*}-\frac{i}{2} \Gamma_{12}^{*}\right)}, \\
& \Gamma_{L}=\Gamma_{11}+2 \operatorname{Im} \sqrt{\left(M_{12}-\frac{i}{2} \Gamma_{12}\right)\left(M_{12}^{*}-\frac{i}{2} \Gamma_{12}^{*}\right)} .
\end{aligned}
$$

Les vecteurs propres de $\mathcal{H}$ sont alors : 


$$
\begin{aligned}
\left|B_{H}\right\rangle & =\frac{p\left|B^{0}\right\rangle+q\left|\bar{B}^{0}\right\rangle}{\sqrt{|p|^{2}+|q|^{2}}} \\
\left|B_{L}\right\rangle & =\frac{p\left|B^{0}\right\rangle-q\left|\bar{B}^{0}\right\rangle}{\sqrt{|p|^{2}+|q|^{2}}}
\end{aligned}
$$

avec :

$$
\frac{p}{q}=\sqrt{\frac{M_{12}^{*}-\frac{i}{2} \Gamma_{12}^{*}}{M_{12}-\frac{i}{2} \Gamma_{12}}} .
$$

Détermination des états propres de $C P$ en fonction des états propres de saveur

Ces états propres physiques ne sont pas forcément états propres de $C P$ et donc il peut y avoir violation de $C P$. Pour déterminer quels sont les états propres de $C P$, il faut imposer la conservation de la symétrie $C P$. Cela implique que $M_{12}$ et $\Gamma_{12}$ soient réels, et donc $p / q=1$. Les états propres de $C P$ sont notés $\left|B_{+}\right\rangle$(associé à la valeur propre de $C P$ +1 ) et $\left|B_{-}\right\rangle$(associé à la valeur propre de $\left.C P-1\right)$ :

$$
\begin{aligned}
\left|B_{+}\right\rangle & =\frac{\left|B^{0}\right\rangle+\left|\bar{B}^{0}\right\rangle}{\sqrt{2}}, \\
\left|B_{-}\right\rangle & =\frac{\left|B^{0}\right\rangle-\left|\bar{B}^{0}\right\rangle}{\sqrt{2}} .
\end{aligned}
$$

\subsubsection{Evolution temporelle des mésons $B$ neutres}

On peut déterminer l'évolution temporelle de $B_{H}$ et $B_{L}$ grâce à l'équation de Schrödinger (équation 1.5), et en utilisant le fait que ce sont des vecteurs propres de $\mathcal{H}$. On obtient ainsi les équations différentielles suivantes :

$$
\begin{aligned}
i \frac{d}{d t}\left|B_{H}(t)\right\rangle & =\left(M_{H}-i \frac{\Gamma_{H}}{2}\right)\left|B_{H}(t)\right\rangle, \\
i \frac{d}{d t}\left|B_{L}(t)\right\rangle & =\left(M_{L}-i \frac{\Gamma_{L}}{2}\right)\left|B_{L}(t)\right\rangle ;
\end{aligned}
$$

dont les solutions sont :

$$
\begin{aligned}
\left|B_{H}(t)\right\rangle & =e^{-\left(i M_{H}+\frac{\Gamma_{H}}{2}\right) t}\left|B_{H}(0)\right\rangle, \\
\left|B_{L}(t)\right\rangle & =e^{-\left(i M_{L}+\frac{\Gamma_{L}}{2}\right) t}\left|B_{L}(0)\right\rangle .
\end{aligned}
$$


Deux paramètres importants pour l'étude des mésons $B$ sont la différence de masse $\Delta m \sim M_{H}-M_{L}$ et la différence de largeur $\Delta \Gamma \sim \Gamma_{H}-\Gamma_{L}$ entre les deux états propres de masse. On les définit de façon à ce que $\Delta m$ soit positif. Cela donne, en fonction des paramètres de $\mathcal{H}$ :

$$
\begin{aligned}
\Delta m & =2 \operatorname{Re} \sqrt{\left(M_{12}-\frac{i}{2} \Gamma_{12}\right)\left(M_{12}^{*}-\frac{i}{2} \Gamma_{12}^{*}\right)}, \\
\Delta \Gamma & =4 \operatorname{Im} \sqrt{\left(M_{12}-\frac{i}{2} \Gamma_{12}\right)\left(M_{12}^{*}-\frac{i}{2} \Gamma_{12}^{*}\right)} .
\end{aligned}
$$

Dans le cas des mésons $B_{d}$, il se trouve que $\Delta \Gamma \ll \Delta m$. En négligeant $\Delta \Gamma$ par rapport à $\Delta m$, on peut en déduire la dépendance temporelle pour un état $\left|B_{\text {phys }}^{0}\right\rangle\left(\left|\bar{B}_{\text {phys }}^{0}\right\rangle\right)$ qui était un pur $\left|B^{0}\right\rangle\left(\left|\bar{B}^{0}\right\rangle\right)$ à $t=0$ :

$$
\begin{aligned}
\left|B_{\text {phys }}^{0}(t)\right\rangle & =e^{-\left(i M t+\frac{\Gamma}{2} t\right)} \cos \left(\Delta m \frac{t}{2}\right)\left|B^{0}\right\rangle+i \frac{q}{p} e^{-\left(i M t+\frac{\Gamma}{2} t\right)} \sin \left(\Delta m \frac{t}{2}\right)\left|\bar{B}^{0}\right\rangle, \\
\left|\bar{B}_{\text {phys }}^{0}(t)\right\rangle & =i \frac{p}{q} e^{-\left(i M t+\frac{\Gamma}{2} t\right)} \sin \left(\Delta m \frac{t}{2}\right)\left|B^{0}\right\rangle+e^{-\left(i M t+\frac{\Gamma}{2} t\right)} \cos \left(\Delta m \frac{t}{2}\right)\left|\bar{B}^{0}\right\rangle .
\end{aligned}
$$

avec $M=\left(M_{H}+M_{L}\right) / 2$ et $\Gamma=\left(\Gamma_{H}+\Gamma_{L}\right) / 2$.

\subsection{3 Évolution temporelle pour $\Upsilon(4 S) \rightarrow B \bar{B}$ dans le cadre de BABAR}

Dans l'expérience BABAR [20], la résonance $\Upsilon(4 S)$ produite par la collision $e^{+} e^{-}$se désintègre en une paire de mésons $B$. On considère ici qu'il s'agit d'une paire de mésons $B$ neutres. $\Upsilon(4 S)$ ayant un spin 1 et les $B$ un spin 0 , la paire de $B$ est produite avec un moment orbital relatif $L=1$. Cela implique que les deux mésons $B$ produits évoluent temporellement en phase selon l'équation 1.19. La probabilité d'avoir deux $B^{0}$ d'impulsions $\vec{p}$ et $-\vec{p}$ (dans le référentiel du centre de masse de $\Upsilon(4 S)$, les deux $B$ sont produits avec des impulsions opposées) avec un moment orbital relatif $L=1$ est [19] :

$$
\left|\left\langle B^{0}(\vec{p}), B^{0}(-\vec{p}) \mid L=1(t)\right\rangle\right|^{2}=0
$$

En effet, l'état $\left|B^{0}(\vec{p}), B^{0}(-\vec{p})\right\rangle$ est symétrique par construction alors que l'état $|L=1(t)\rangle$ est antisymétrique : le produit scalaire est donc nul. De la même manière, la probabilité d'avoir deux $\bar{B}^{0}$ est nulle. On en déduit donc que tant qu'aucun des deux $B$ ne s'est désintégré, il y a toujours exactement un $B^{0}$ et un $\bar{B}^{0}$ dans l'événement, et ce, malgré l'oscillation des deux $B$. Une fois qu'une des particules s'est désintégrée, l'autre continue 
à évoluer. C'est pourquoi, il peut y avoir des événements avec un $B^{0}$ et un $\bar{B}^{0}$, des événements avec deux $B^{0}$ ou des événements avec deux $\bar{B}^{0}$. La probabilité d'obtenir l'un de ces cas de figure dépend de la différence de temps $\Delta t$ entre les désintégrations des deux $B$.

Cette spécificité est très intéressante pour l'étude de la désintégration $B \rightarrow f$. On sélectionne les événements dans lesquels l'un des deux $B\left(B_{\text {rec }}\right)$ s'est désintégré en un état final $f$. L'autre $B\left(B_{t a g}\right)$ sert à étiqueter l'événement : on détermine en effet la saveur de $B_{\text {tag }}$ par la reconstruction inclusive de ses produits de désintégration. On connaît alors la saveur de $B_{r e c}$ à l'instant $t_{t a g}$ de la désintégration de $B_{t a g}$. Connaissant l'évolution temporelle de $B_{\text {rec }}$ entre $t_{t a g}$ et l'instant de sa désintégration $t_{r e c}$, on peut en déduire la saveur de $B_{\text {rec }}$ au moment de sa désintégration en $f$. La probabilité de la transition d'un $B$ en $f$ est donnée par $\left|\left\langle f|\mathcal{T}| B_{\text {phys }}^{0}(\Delta t)\right\rangle\right|^{2}$ ou $\left|\left\langle f|\mathcal{T}| \bar{B}_{\text {phys }}^{0}(\Delta t)\right\rangle\right|^{2}$; avec $\Delta t=t_{\text {rec }}-t_{\text {tag }}$. D'après l'équation 1.19 , cette probabilité est :

$$
\begin{aligned}
\left|\left\langle f|\mathcal{T}| B_{\text {phys }}^{0}(\Delta t)\right\rangle\right|^{2}= & \frac{e^{-\Gamma|\Delta t|}}{2}\left[(1+\cos (\Delta m \Delta t))\left|\left\langle f|\mathcal{T}| B^{0}\right\rangle\right|^{2}\right. \\
& +(1-\cos (\Delta m \Delta t))\left|\frac{q}{p}\right|^{2}\left|\left\langle f|\mathcal{T}| \bar{B}^{0}\right\rangle\right|^{2} \\
& \left.+2 \operatorname{Im}\left(\sin (\Delta m \Delta t)\left(\frac{q}{p}\right)^{*}\left\langle f|\mathcal{T}| B^{0}\right\rangle\left\langle f|\mathcal{T}| \bar{B}^{0}\right\rangle^{*}\right)\right]
\end{aligned}
$$

et pour $\left|\left\langle f|\mathcal{T}| \bar{B}_{\text {phys }}^{0}(t)\right\rangle\right|^{2}$ :

$$
\begin{aligned}
\left|\left\langle f|\mathcal{T}| \bar{B}_{\text {phys }}^{0}(\Delta t)\right\rangle\right|^{2}= & \frac{e^{-\Gamma|\Delta t|}}{2}\left[(1+\cos (\Delta m \Delta t))\left|\left\langle f|\mathcal{T}| \bar{B}^{0}\right\rangle\right|^{2}\right. \\
& +(1-\cos (\Delta m \Delta t))\left|\frac{p}{q}\right|^{2}\left|\left\langle f|\mathcal{T}| B^{0}\right\rangle\right|^{2} \\
& \left.+2 \operatorname{Im}\left(\sin (\Delta m \Delta t)\left(\frac{p}{q}\right)^{*}\left\langle f|\mathcal{T}| \bar{B}^{0}\right\rangle\left\langle f|\mathcal{T}| B^{0}\right\rangle^{*}\right)\right] .
\end{aligned}
$$

On obtient des équations similaires pour l'état final $|\bar{f}\rangle=C P|f\rangle$. La comparaison de $\left|\left\langle f|\mathcal{T}| B_{\text {phys }}^{0}(\Delta t)\right\rangle\right|^{2}$ et $\left|\left\langle\bar{f}|\mathcal{T}| \bar{B}_{\text {phys }}^{0}(\Delta t)\right\rangle\right|^{2}$ ou de $\left|\left\langle\bar{f}|\mathcal{T}| B_{\text {phys }}^{0}(\Delta t)\right\rangle\right|^{2}$ et $\left|\left\langle f|\mathcal{T}| \bar{B}_{\text {phys }}^{0}(\Delta t)\right\rangle\right|^{2}$ peut mettre en évidence des effets de violation de $C P$.

On définit deux paramètres complexe $\lambda$ et $\bar{\lambda}$ :

$$
\lambda \equiv \frac{q}{p} \frac{\left\langle f|\mathcal{T}| \bar{B}^{0}\right\rangle}{\left\langle f|\mathcal{T}| B^{0}\right\rangle}, \quad \quad \bar{\lambda} \equiv \frac{p}{q} \frac{\left\langle\bar{f}|\mathcal{T}| B^{0}\right\rangle}{\left\langle\bar{f}|\mathcal{T}| \bar{B}^{0}\right\rangle} .
$$

Avec cette notation, les équations 1.21 et 1.22 s'écrivent :

$$
\begin{aligned}
\left|\left\langle f|\mathcal{T}| B_{\text {phys }}^{0}(\Delta t)\right\rangle\right|^{2}= & \frac{e^{-\Gamma|\Delta t|}}{2}\left|\left\langle f|\mathcal{T}| B^{0}\right\rangle\right|^{2}[(1+\cos (\Delta m \Delta t)) \\
& +(1-\cos (\Delta m \Delta t))|\lambda|^{2} \\
& -2 \operatorname{Im}(\lambda) \sin (\Delta m \Delta t)]
\end{aligned}
$$




$$
\begin{aligned}
\left|\left\langle f|\mathcal{T}| \bar{B}_{\text {phys }}^{0}(\Delta t)\right\rangle\right|^{2}= & \frac{e^{-\Gamma|\Delta t|}}{2}\left|\left\langle f|\mathcal{T}| \bar{B}^{0}\right\rangle\right|^{2}[(1+\cos (\Delta m \Delta t)) \\
& +(1-\cos (\Delta m \Delta t))|\lambda|^{-2} \\
& \left.-2 \operatorname{Im}\left(\lambda^{-1}\right) \sin (\Delta m \Delta t)\right]
\end{aligned}
$$

\subsubsection{Les trois types de violation de $C P$}

\section{La violation de $C P$ dans les désintégrations}

La violation de $C P$ dans les désintégrations est la plus intuitive des violations de $C P$. Elle se manifeste tout simplement par une différence entre une amplitude de transition et l'amplitude de transition conjuguée. C'est pourquoi on l'appelle également violation de $C P$ directe. On note $\mathcal{A}_{f}$ l'amplitude de transition $B^{0} \rightarrow f$ et $\overline{\mathcal{A}}_{\bar{f}}$ l'amplitude conjuguée (transition $\left.\bar{B}^{0} \rightarrow \bar{f}\right)$. Il y a violation de $C P$ dans les désintégrations si $\mathcal{A}_{f} \neq \overline{\mathcal{A}}_{\bar{f}}$.

Pour étudier la violation de $C P$ directe, il est intéressant de considérer la quantité $\left|\frac{\mathcal{A}_{f}}{\overline{\mathcal{A}}_{\bar{f}}}\right|$, qui est indépendante de toute convention de phase, et qui est donc physiquement intéressante.

Il existe deux types de phases dans $\mathcal{A}_{f}$ et $\overline{\mathcal{A}}_{\bar{f}}$ :

- les phases des constantes de couplage, que l'on appelle phases faibles, car elles sont générées par la matrice CKM, qui est gouvernée par l'interaction faible. Les phases faibles de $\mathcal{A}_{f}$ et $\overline{\mathcal{A}}_{\bar{f}}$ ont des signes opposés. On peut remarquer que si la phase faible d'un terme isolé du Lagrangien dépend d'une convention de phase, ce n'est pas le cas pour la différence entre les phases faibles de deux termes du Lagrangien. Cette différence est, elle, indépendante de la convention de phase choisie.

- les phases de tous les processus intermédiaires réels qui contribuent à la transition $B^{0} \rightarrow f$, que l'on appelle phases fortes, car elles correspondent à des processus de diffusion qui sont dominés par l'interaction forte. Ces phases ne violent par $C P$ et sont donc égales pour $\mathcal{A}_{f}$ et $\overline{\mathcal{A}}_{\bar{f}}$. La quantité physique intéressante est également la différence de phases fortes entre deux termes du Lagrangien car elle est indépendante de la convention de phase.

L'amplitude de transition $\mathcal{A}_{f}$ est la somme des amplitudes des différentes contributions $\mathcal{A}_{i}$ au processus $B^{0} \rightarrow f$. On peut définir chacune de ses amplitudes par son module $\left|\mathcal{A}_{i}\right|$, sa phase forte $\delta_{i}$ et sa phase faible $\phi_{i}$. On peut donc écrire :

$$
\mathcal{A}_{f}=\sum_{i}\left|\mathcal{A}_{i}\right| e^{i\left(\delta_{i}+\phi_{i}\right)}, \quad \overline{\mathcal{A}}_{\bar{f}}=\sum_{i}\left|\mathcal{A}_{i}\right| e^{i\left(\delta_{i}-\phi_{i}\right)}
$$

On en déduit : 


$$
\left|\frac{\mathcal{A}_{f}}{\overline{\mathcal{A}}_{\bar{f}}}\right|=\left|\frac{\sum_{i}\left|\mathcal{A}_{i}\right| e^{i\left(\delta_{i}+\phi_{i}\right)}}{\sum_{i}\left|\mathcal{A}_{i}\right| e^{i\left(\delta_{i}-\phi_{i}\right)}}\right| .
$$

Si $C P$ est conservée, toutes les phases faibles sont nulles et donc $\left|\frac{\mathcal{A}_{f}}{\overline{\mathcal{A}}_{\bar{f}}}\right|=1$. Par contraposée, on obtient que si $\left|\frac{\mathcal{A}_{f}}{\overline{\mathcal{A}_{\bar{f}}}}\right| \neq 1$, alors il y a violation de $C P$.

On remarque que cette violation directe de $C P$ résulte d'interférences violant $C P$ entre les différents termes des amplitudes de désintégrations. Pour mieux comprendre, plaçonsnous dans le cas simple où il n'y a que deux processus qui contribuent à la transition $B^{0} \rightarrow f$. On note $\mathcal{A}_{1}=\left|\mathcal{A}_{1}\right| e^{i\left(\delta_{1}+\phi_{1}\right)}$ et $\mathcal{A}_{2}=\left|\mathcal{A}_{2}\right| e^{i\left(\delta_{2}+\phi_{2}\right)}$ ces deux amplitudes. On a done :

$$
\begin{aligned}
& \mathcal{A}_{f}=\left|\mathcal{A}_{1}\right| e^{i\left(\delta_{1}+\phi_{1}\right)}+\left|\mathcal{A}_{2}\right| e^{i\left(\delta_{2}+\phi_{2}\right)}, \\
& \overline{\mathcal{A}}_{\bar{f}}=\left|\mathcal{A}_{1}\right| e^{i\left(\delta_{1}-\phi_{1}\right)}+\left|\mathcal{A}_{2}\right| e^{i\left(\delta_{2}-\phi_{2}\right)} .
\end{aligned}
$$

On définit une asymétrie de $C P, a_{f}$, qui rend compte de la différence éventuelle entre la probabilité de transition $B^{0} \rightarrow f$ et la probabilité de transition conjuguée $\bar{B}^{0} \rightarrow \bar{f}$ :

$$
\begin{aligned}
a_{f} & =\frac{\mathcal{P}\left(B^{0} \rightarrow f\right)-\mathcal{P}\left(\bar{B}^{0} \rightarrow \bar{f}\right)}{\mathcal{P}\left(B^{0} \rightarrow f\right)+\mathcal{P}\left(\bar{B}^{0} \rightarrow \bar{f}\right)}=\frac{\mathcal{A}_{f}^{2}-\overline{\mathcal{A}}_{\bar{f}}^{2}}{\mathcal{A}_{f}^{2}+\overline{\mathcal{A}}_{\bar{f}}^{2}} \\
& =\frac{-2\left|\mathcal{A}_{1}\right|\left|\mathcal{A}_{2}\right| \sin \left(\phi_{1}-\phi_{2}\right) \sin \left(\delta_{1}-\delta_{2}\right)}{\left|\mathcal{A}_{1}\right|^{2}+\left|\mathcal{A}_{2}\right|^{2}+2\left|\mathcal{A}_{1}\right|\left|\mathcal{A}_{2}\right| \cos \left(\phi_{1}-\phi_{2}\right) \cos \left(\delta_{1}-\delta_{2}\right)}
\end{aligned}
$$

Pour qu'il y ait violation de $C P$, il faut donc que $\phi_{1} \neq \phi_{2}$ et $\delta_{1} \neq \delta_{2}$. En résumé, il peut y avoir violation de $C P$ dans les désintégrations s'il y a aux moins deux termes qui contribuent à l'amplitude de transition avec des phases faibles et fortes différentes.

\section{La violation de $C P$ dans le mélange}

Il y a violation de $C P$ dans le mélange (ou violation de $C P$ indirecte) si la probabilité d'oscillation de $B^{0}$ en $\bar{B}^{0}$ est différente de la probabilité d'oscillation du $\bar{B}^{0}$ en $B^{0}$. C'est possible si les états propres de masse $B_{H}$ et $B_{L}$ ne sont pas états propres de $C P$, ce qui revient à dire que :

$$
\left|\frac{p}{q}\right|^{2} \neq 1
$$

On a d'après la relation suivante :

$$
\left|\frac{p}{q}\right|^{2}=\left|\frac{M_{12}^{*}-\frac{i}{2} \Gamma_{12}^{*}}{M_{12}-\frac{i}{2} \Gamma_{12}}\right| .
$$


Ce rapport est différent de 1 si la phase relative entre $M_{12}$ et $\Gamma_{12}$ est différente de 0 ou $\pi$.

Le Modèle Standard prédit que la violation de $C P$ dans le mélange est très faible. Il est cependant possible d'observer cette violation de $C P$ en utilisant un mode autoétiquetant du $B$, afin de connaître à la fois la saveur de $B_{t a g}$ et de $B_{r e c}$. Un bon mode de désintégration est la désintégration semi-leptonique : $B \rightarrow l^{-} \nu X$. Le signe du lepton permet de déterminer la saveur du $B$ neutre.

La violation de $C P$ dans le mélange montre que $\mathcal{A}\left(B^{0} \rightarrow \bar{B}^{0}\right) \neq \mathcal{A}\left(\bar{B}^{0} \rightarrow B^{0}\right)$. Tester cette asymétrie permet donc également de tester la violation de $T$.

\section{La violation de $C P$ dans les interférences entre les désintégrations avec et sans mélange}

Ce troisième type de violation de $C P$ est possible quand un état final est à la fois accessible par la désintégration de $B^{0}$ et de $\bar{B}^{0}$.

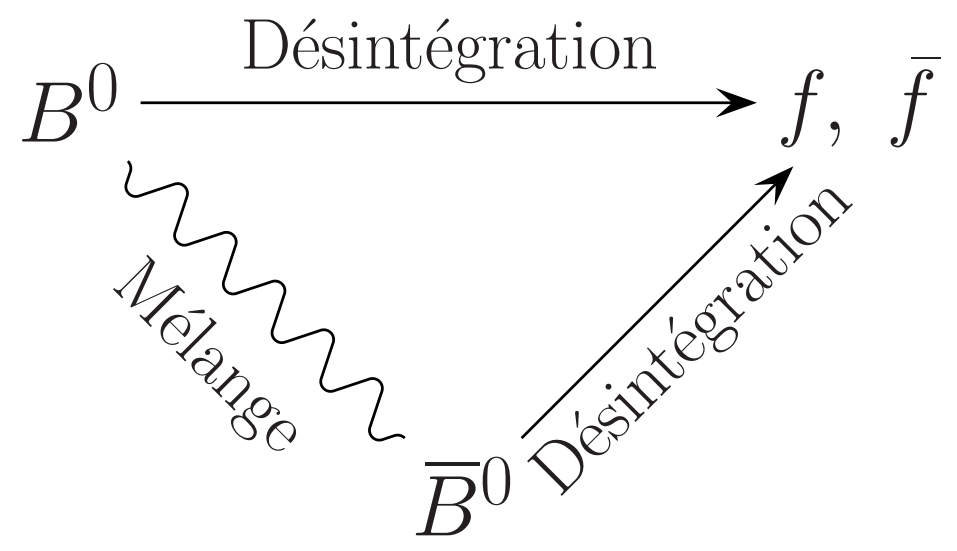

Pour simplifier le formalisme, considérons le cas où l'état final est état propre de $C P$ avec $\eta_{C P}=+1$. On le note $f_{C P}$. La quantité intéressante à considérer est $\lambda$ (équation 1.23), car elle est indépendante de phase et a du sens physiquement. Avec les notations utilisées ici, on peut écrire :

$$
\lambda=\frac{q}{p} \frac{\mathcal{A}_{f}}{\overline{\mathcal{A}}_{\bar{f}}} .
$$

La violation de $C P$ se traduit par $\lambda \neq \pm 1$. On peut remarquer que s'il y a violation de $C P$ dans les désintégrations ou dans le mélange, alors $\lambda \neq \pm 1$. Cependant, il est également possible que $|q / p|=1$ et $\left|\mathcal{A}_{f}\right| \overline{\mathcal{A}}_{\bar{f}} \mid=1$ avec une très bonne approximation, mais que pourtant $\lambda \neq \pm 1$. Dans ce cas là, on a $|\lambda|=1$ et $\operatorname{Im}(\lambda) \neq 0$. Ce troisième type de violation de $C P$ est dû à la phase relative entre $q / p$ et $\mathcal{A}_{f} / \overline{\mathcal{A}}_{\bar{f}}$ et peut être mis en évidence expérimentalement si on mesure $\operatorname{Im}(\lambda) \neq 0$.

L'asymétrie de $C P$ dépendante du temps s'écrit de manière générale :

$$
a_{C P}(\Delta t)=\frac{\left|\left\langle f_{C P}|\mathcal{T}| B_{\text {phys }}^{0}(\Delta t)\right\rangle\right|^{2}-\left|\left\langle f_{C P}|\mathcal{T}| \bar{B}_{\text {phys }}^{0}(\Delta t)\right\rangle\right|^{2}}{\left|\left\langle f_{C P}|\mathcal{T}| B_{\text {phys }}^{0}(\Delta t)\right\rangle\right|^{2}+\left|\left\langle f_{C P}|\mathcal{T}| \bar{B}_{\text {phys }}^{0}(\Delta t)\right\rangle\right|^{2}}
$$


En utilisant les équations 1.24 et $1.25, a_{C P}(\Delta t)$ s'écrit en fonction de $\lambda$ :

$$
a_{C P}(\Delta t)=\frac{\left(1-|\lambda|^{2}\right) \cos (\Delta m \Delta t)-2 \operatorname{Im}(\lambda) \sin (\Delta m \Delta t)}{1+|\lambda|^{2}} .
$$

Si l'un des trois types de violation de $C P$ décrits précédemment est présent dans le mode que l'on étudie, alors $a_{C P} \neq 0$. Le coefficient $\left(1-|\lambda|^{2}\right)$, qui est devant le terme en $\cos (\Delta m \Delta t)$, est sensible à la fois à la violation de $C P$ dans le mélange et à la violation de $C P$ directe. En effet, ces deux types de violation de $C P$ impliquent $|\lambda| \neq 1$ et donc le coefficient devant le terme $\cos (\Delta m \Delta t)$ n'est pas nul. Cependant, puisque la violation de $C P$ dans le mélange est très faible, on dit souvent par abus de language que si on mesure un coefficient significativement différent de 0 devant le terme $\cos (\Delta m \Delta t)$, alors il y a violation de $C P$ directe. Le coefficient $\operatorname{Im}(\lambda)$ devant le terme $\sin (\Delta m \Delta t)$ est quant à lui sensible à la violation de $C P$ dans l'interférence entre la désintégration et le mélange.

Si on néglige la violation de $C P$ directe et la violation de $C P$ dans le mélange, ce que l'on peut souvent faire avec une bonne approximation, on a $|\lambda|=1$ et l'expression de $a_{C P}$ simplifiée s'écrit :

$$
a_{C P}(\Delta t)=-\operatorname{Im}(\lambda) \sin (\Delta m \Delta t)
$$

Cette asymétrie est observable, car le signe du coefficient devant le terme $\sin (\Delta m \Delta t)$ n'est pas le même dans le cas de la désintégration du $B^{0}$ en $f$ (équation 1.24) et dans le cas de la désintégration du $\bar{B}^{0}$ en $f$ (équation 1.25).

Dans le cas idéal où il n'y a qu'une seule amplitude qui intervient, il est possible de relier $a_{C P}$ de façon propre aux paramètres du Lagrangien afin de mesurer les angles du triangle d'unitarité, comme on le verra dans la suite. En réalité, il existe souvent d'autres contributions, comme par exemple des diagrammes en boucle, qui font intervenir des phases supplémentaires. Si ces phases sont différentes des phases introduites par le diagramme principal, on ne peut alors plus relier proprement la mesure du coefficient devant le terme en $\sin (\Delta m \Delta t)$ aux phases de la matrice CKM. Ces contributions supplémentaires peuvent parfois être négligées, mais il existe des modes de désintégrations du $B^{0}$ pour lesquels il est impossible de les négliger. Si plusieurs amplitudes avec des phases faibles différentes contribuent au processus, $a_{C P}$ dépend également du rapport entre les différentes amplitudes et de leurs phases fortes relatives. Il peut alors y avoir de nombreuses incertitudes hadroniques sur les prédictions. Ce point sera développé dans la suite.

\subsection{La violation de $C P$ dans le cadre du Modèle Stan- dard}

Cette partie explique de quelle façon la violation de $C P$ est générée dans le Modèle Standard [21,22]. Le Modèle Standard est basé sur le groupe de jauge $S U(3)_{C} \otimes S U(2)_{L} \otimes U(1)_{Y}$, où $C, L$ et $Y$ représentent respectivement la couleur, l'isospin faible et l'hypercharge faible. On s'intéresse ici plus particulièrement à l'interaction faible, car c'est la seule interaction qui ne conserve pas la saveur et qui permette donc la désintégration des $B$. De plus, le phénomène de violation de $C P$ aurait lieu via cette interaction. Notons cependant que 
la situation est compliquée par le fait que les quarks constituant les mésons sont également soumis à l'interaction forte. Cela peut rendre l'interprétation de certaines mesures expérimentales relativement ardue. Avant de décrire la violation de $C P$ dans le cadre du Modèle Standard, il est important de préciser que si le Modèle Standard rend bien compte jusqu'à présent des observations expérimentales dans le secteur de la violation de $C P$, il ne permet pas d'expliquer l'origine de la violation de $C P$.

\subsubsection{Interaction faible et violation de $C P$}

L'interaction faible est décrite par le groupe de jauge $S U(2)_{L} \otimes U(1)_{Y}$. Les composantes gauches des champs de fermions ${ }^{2}$ :

$$
\left(\begin{array}{c}
\nu_{i} \\
l_{i}^{-}
\end{array}\right)_{G} \quad\left(\begin{array}{c}
u_{i} \\
d_{i}
\end{array}\right)_{G}
$$

se transforment comme des doublets sous $S U(2)$, alors que les composantes droites:

$$
\left(l_{i}^{-}\right)_{D} \quad\left(u_{i}\right)_{D} \quad\left(d_{i}\right)_{D}
$$

se transforment comme des singlets. Ici, $i$ représente la famille, $\nu$ un neutrino, $l$ un lepton, $u$ un quark de type up et $d$ un quark de type down.

Lors de la brisure de symétrie électrofaible, le champ de Higgs [23] confère une masse à chaque fermion à travers le couplage de Yukawa. Le lagrangien est (après la brisure de symétrie) :

$$
\mathcal{L}_{Y}=-\left(\overline{\mathbf{u}}_{L}^{\prime} \mathbf{m} \mathbf{u}_{R}^{\prime}+\overline{\mathbf{d}}_{L}^{\prime} \widetilde{\mathbf{m}} \mathbf{d}_{R}^{\prime}+\text { h.c. }\right)\left(1+\frac{\phi_{0}}{v}\right) .
$$

Dans cette équation :

- $\phi_{0}$ représente le champ de Higgs scalaire et $v$ est sa valeur moyenne dans le vide;

- $\mathbf{u}_{R, L}^{\prime}$ et $\mathbf{d}_{R, L}^{\prime}$ sont les vecteurs de quarks up et down dans l'espace des saveurs à trois dimensions :

$$
\mathbf{u}_{R, L}^{\prime}=\left(\frac{1 \mp \gamma_{5}}{2}\right)\left(\begin{array}{c}
u^{\prime} \\
c^{\prime} \\
t^{\prime}
\end{array}\right), \quad \quad \mathbf{d}_{R, L}^{\prime}=\left(\frac{1 \mp \gamma_{5}}{2}\right)\left(\begin{array}{c}
d^{\prime} \\
s^{\prime} \\
b^{\prime}
\end{array}\right)
$$

- $\mathbf{m}$ et $\widetilde{\mathbf{m}}$ sont des matrices de masse complexes $3 \times 3$;

Les matrices $\mathbf{m}$ et $\widetilde{\mathbf{m}}$ ne sont pas diagonales. Physiquement, cela veut dire que les états propres de saveur des quarks ne sont pas états propres de masse. Avec les notations utilisées ici, les états propres de saveur sont notés $q^{\prime}$ et les états propres de masse (ou états propres physiques) sont notés $q$. On peut passer d'une représentation à l'autre par un changement de base :

$$
\mathbf{u}_{R, L}=V_{L, R} \mathbf{u}_{R, L}^{\prime}, \quad \mathbf{d}_{R, L}=\widetilde{V}_{L, R} \mathbf{d}_{R, L}^{\prime}
$$

${ }^{2}$ particules de matière de spin $1 / 2$ 
$V_{L, R}$ et $\widetilde{V}_{L, R}$ sont des matrices unitaires de changement de base qui diagonalisent $\mathbf{m}$ et $\widetilde{\mathbf{m}}$.

Le couplage des quarks avec les bosons $W^{ \pm}$introduit le mélange entre les familles. Le Lagrangien correspondant est :

$$
\mathcal{L}_{W}=\frac{g}{\sqrt{2}}\left(\overline{\mathbf{u}}_{L} \gamma^{\mu} W_{\mu}^{+} V_{C K M} \mathbf{d}_{L}+\overline{\mathbf{d}}_{L} \gamma^{\mu} W_{\mu}^{-} V_{C K M}^{\dagger} \mathbf{u}_{L}\right)
$$

où $V_{C K M}=V_{L} \widetilde{V}_{L}^{\dagger}$ est la matrice de mélange des quarks, appelée également matrice CKM (Cabibbo-Kobayashi-Maskawa) [24,25]. Par convention, les états propres de saveur sont les états propres de masse pour les quarks up $(u, c, t)$. Avec cette convention, qui n'est rien d'autre qu'une convention de phase, les quarks up ne se mélangent pas. En revanche, les quarks down $(d, s, b)$ ne sont pas états propres de saveur. Les états physiques de ces quarks sont reliés aux états de saveur par la matrice de CKM, qui est unitaire et a priori complexe :

$$
V_{C K M}=\left(\begin{array}{ccc}
V_{u d} & V_{u s} & V_{u b} \\
V_{c d} & V_{c s} & V_{c b} \\
V_{t d} & V_{t s} & V_{t b}
\end{array}\right)
$$

Chaque terme $V_{i j}$ de la matrice représente la probabilité de transition du quark $i$ vers le quark $j$. Notons que quelque soit la paramétrisation choisie, l'amplitude du couplage entre deux quarks $i$ et $j$ est invariante et est égale à $\left|V_{i j}\right|$.

\subsubsection{La matrice CKM}

Cette matrice étant unitaire, il est possible de l'exprimer en fonction d'un produit de matrices de rotation. Une matrice unitaire de dimensions $N \times N$ comporte $N^{2}$ paramètres indépendants : $\frac{N(N-1)}{2}$ angles de rotation et $\frac{N(N+1)}{2}$ phases. $V_{C K M}$ étant de dimension $3 \times 3$, elle peut être décrite par trois angles de rotations et six phases. Il est cependant possible de trouver une convention de phase telle que cinq phases soient absorbées. En effet, la paramétrisation qui supprime la maximum de paramètres complexes comporte trois angles de mélange $\theta_{12}, \theta_{23}$ et $\theta_{13}$ et une phase $\delta$. Notons que dans le cadre du Modèle Standard avec un doublet de Higgs, il faut au moins trois familles pour que cette phase soit non triviale, c'est-à-dire pour qu'elle ne disparaisse pas par changement de phase. On peut remarquer que pour que cette phase soit irréductible, il faut que les masses des quarks de même charge ne soient pas dégénérées. Cela se traduit par la relation suivante :

$$
\left(m_{t}^{2}-m_{c}^{2}\right)\left(m_{c}^{2}-m_{u}^{2}\right)\left(m_{t}^{2}-m_{u}^{2}\right)\left(m_{b}^{2}-m_{s}^{2}\right)\left(m_{s}^{2}-m_{d}^{2}\right)\left(m_{b}^{2}-m_{d}^{2}\right) \neq 0 .
$$

Notons que dans cette relation, c'est la masse au carré des quarks qui apparaît, car le signe d'un terme de masse d'un fermion n'a pas de signification physique. Cette condition est nécessaire, car si deux quarks de même charge avaient la même masse, une nouvelle symétrie (rotation unitaire dans l'espace de deux quarks de masse dégénérée) apparaitrait dans le Lagrangien et supprimerait la phase complexe. 
S'il n'y avait que deux familles, la matrice serait réelle et pourrait être paramétrée par un seul angle de rotation. Ainsi en 1963, à l'époque où l'on ne connaissait que les quarks $u, d$ et $s$, Cabibbo avait introduit cet angle de rotation $\theta_{C}$ (appelé angle de Cabibbo) pour expliquer la désintégration des particules étranges [24]. En 1973, Kobayashi et Maskawa émirent l'idée qu'avec une troisième génération de quarks, la matrice pouvait être complexe, permettant ainsi la violation de $C P$ [25].

C'est la présence de cette phase non triviale qui permet de générer la violation de $C P$ à travers des interférences entre deux diagrammes mettant en jeu différents éléments de la matrice. La matrice $V_{C K M}$ peut s'exprimer ainsi en fonction de ces quatre paramètres fondamentaux [26] :

$$
V_{C K M}=\left(\begin{array}{ccc}
C_{12} C_{13} & S_{12} C_{13} & S_{13} e^{-i \delta} \\
-S_{12} C_{23}-C_{12} S_{23} S_{13} e^{i \delta} & C_{12} C_{23}-S_{12} S_{23} S_{13} e^{i \delta} & S_{23} C_{13} \\
S_{12} S_{23}-C_{12} C_{23} S_{13} e^{i \delta} & -C_{12} S_{23}-S_{12} C_{23} S_{13} e^{i \delta} & C_{23} C_{13}
\end{array}\right)
$$

avec $C_{i j}=\cos \theta_{i j}$ et $S_{i j}=\sin \theta_{i j}$.

\subsubsection{Paramétrisation de Wolfenstein}

Une des paramétrisations les plus utilisées est la paramétrisation de Wolfenstein [27], qui dépend de quatre paramètres réels : $\lambda=\cos \theta_{12}, A=\sin \theta_{23} / \lambda^{2}, \rho=\sin \theta_{13} /\left(A \lambda^{3}\right) \cos \delta$ et $\eta=\sin \theta_{13} /\left(A \lambda^{3}\right) \sin \delta$. Ici, $\theta_{12}$ représente l'angle de Cabibbo. $\lambda$ est de l'ordre de 0,22 . A l'ordre $\lambda^{4}$, l'expression de $V_{C K M}$ avec cette paramétrisation est :

$$
V_{C K M}=\left(\begin{array}{ccc}
1-\frac{\lambda^{2}}{2} & \lambda & A \lambda^{3}(\rho-i \eta) \\
-\lambda & 1-\frac{\lambda^{2}}{2} & A \lambda^{2} \\
A \lambda^{3}(1-\rho-i \eta) & -A \lambda^{2} & 1
\end{array}\right)+\mathcal{O}\left(\lambda^{4}\right) .
$$

Cette représentation est bien adaptée à la hiérarchie des éléments de matrice et permet de bien visualiser les contraintes expérimentales. Les termes diagonaux sont d'ordre 1, ce qui traduit le fait que $d^{\prime} \sim d, s^{\prime} \sim s$ et $b^{\prime} \sim b$, et les termes non-diagonaux sont en première approximation en puissance de $\lambda$. Comme l'amplitude de transition d'un quark à un autre est proportionnelle au module des éléments de matrice, on peut estimer les probabilités de transition relatives entre les différents quarks. Plus on s'éloigne de la diagonale, plus les termes sont petits. En effet, le mélange entre les générations 1 et 2 est plus important que celui entre les générations 2 et 3, qui est lui-même plus important qu'entre les générations 1 et 3 .

La phase complexe, paramétrée par $\rho$ et $\eta$ n'est présente que dans deux termes de la matrice à l'ordre $\lambda^{4}$, dans cette convention de phase.

\subsubsection{Unitarité de la matrice CKM}

L'unitarité de la matrice $\left(V^{+} V=1\right)$ requière que pour tout choix de $i, j, k, l=1,2,3$, on ait : 


$$
\Im\left[V_{i j} V_{k l} V_{i l}^{*} V_{k j}^{*}\right]=J \sum_{m, n=1}^{3} \epsilon_{i k m} \epsilon_{j l n} .
$$

où $J$ est l'invariant de Jarlskog [28]. Les conditions nécessaires à la violation de $C P$ sur les paramètres de mélange peuvent être résumées ainsi : $J \neq 0$.

Toutes les conditions nécessaires à la violation de $C P$ peuvent être regroupées en une seule condition sur les matrices de masse dans la base des interactions :

$$
\Im\left\{\operatorname{det}\left[\widetilde{\mathbf{m}} \widetilde{\mathbf{m}}^{\dagger}, \mathbf{m m}^{\dagger}\right]\right\} \neq 0 \Leftrightarrow \text { violation de } C P .
$$

Cette condition est indépendante de toute convention. L'invariant de Jarlskog $J$ permet de quantifier l'importance de la violation de $C P$. En principe, la valeur maximale que $J$ peut atteindre est $1 /(6 \sqrt{3}) \approx 0.1$, mais la valeur de $J$ est en réalité de l'ordre de $10^{-5}$, ce qui est révélateur de la faible intensité de la violation de $C P$ dans le cadre du Modèle Standard.

Avec la paramétrisation de l'équation 1.43, $J=c_{12} c_{23} c_{13}^{2} s_{12} s_{23} s_{13} \sin \delta$. Pour qu'il y ait violation de $C P$, il faut donc que les angles de mélange soient différents de 0 ou $\pi / 2$ et que $\delta$ soit différent de 0 ou $\pi$. Dans la paramétrisation de Wolfenstein, $J=A^{2} \lambda^{6} \eta$ et la condition pour qu'il y ait violation de $C P$ se traduit par $\eta \neq 0$.

L'unitarité de la matrice de CKM implique également des relations entre les différents éléments de la matrice :

$$
\begin{aligned}
V_{u d} V_{u s}^{*}+V_{c d} V_{c s}^{*}+V_{t d} V_{t s}^{*} & =0, \\
V_{u s} V_{u b}^{*}+V_{c s} V_{c b}^{*}+V_{t s} V_{t b}^{*} & =0, \\
V_{u d} V_{u b}^{*}+V_{c d} V_{c b}^{*}+V_{t d} V_{t b}^{*} & =0 .
\end{aligned}
$$

Comme toute somme de trois termes complexes égale à 0 , ces relations peuvent être représentées par des triangles dans le plan complexe. Ces relations sont relatives respectivement aux diagrammes en boîtes décrivant le mélange $K^{0} \rightarrow \bar{K}^{0}, B_{s} \rightarrow \bar{B}_{s}$ et $B^{0} \rightarrow \bar{B}^{0}$. Ils sont représentés sur la figure 1.2.

Les deux premiers triangles sont très plats. Le troisième, en revanche, a des côtés du même ordre de grandeur, ce qui rend ses paramètres mesurables expérimentalement. Les mesures de ses angles et côtés, qui est l'un des principaux objectifs de BABAR est donc expérimentalement possible. Le fait qu'il n'y ait qu'une seule phase complexe non triviale implique que les trois triangles ont la même aire. Celle-ci est égale à $J / 2$.

Les angles $\alpha, \beta$ et $\gamma$ de ce triangle, appelé "triangle d'unitarité" et représenté sous deux formes par la figure 1.3, peuvent être mesurés en étudiant la violation de $C P$ dans les mésons $B$, et sont directement reliés aux phases faibles du Lagrangien. On a les relations suivantes :

$$
\alpha \equiv \arg \left[-\frac{V_{t d} V_{t b}^{*}}{V_{u d} V_{u b}^{*}}\right], \quad \beta \equiv \arg \left[-\frac{V_{c d} V_{c b}^{*}}{V_{t d} V_{t b}^{*}}\right], \quad \gamma \equiv \arg \left[-\frac{V_{u d} V_{u b}^{*}}{V_{c d} V_{c b}^{*}}\right] .
$$


(a)

(b)

7-92

(c)

7204A4

Fig. 1.2: Les trois triangles correspondant aux relations de l'équation 1.47.

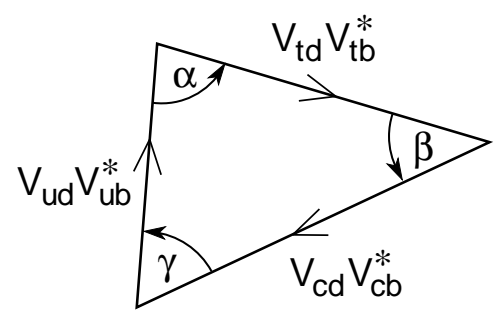

(a)

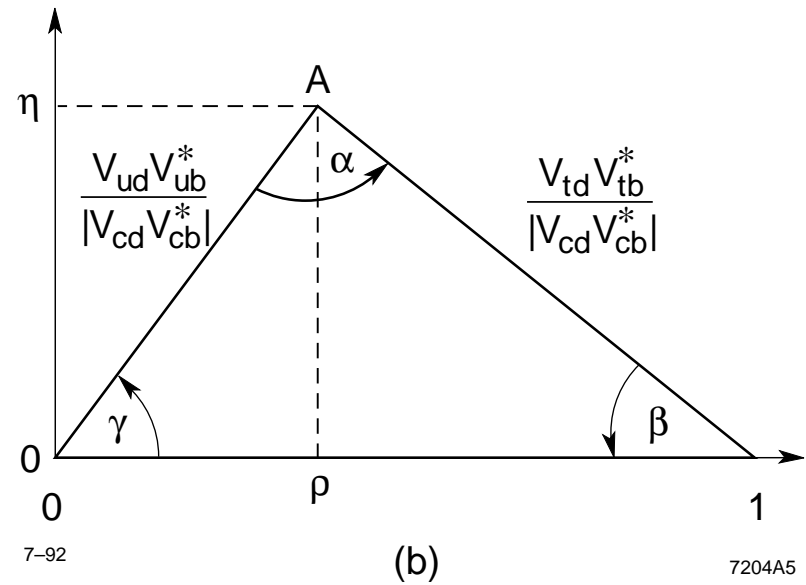

FIG. 1.3: Le premier triangle est la représentation de l'équation d'unitarité relative aux $B_{d}$. Le deuxième triangle est relatif à la même relation, mais est représenté avec une convention de phase telle que $V_{c d} V_{c b}^{*}$ soit réel. De plus, il a été renormalisé de façon à ce que ce côté ait pour longueur 1.

Il est important de mesurer tous les paramètres (angles et côtés) du triangle, afin de tester la cohérence du Modèle Standard. La violation de $C P$ n'est générée que par une seule phase dans ce cadre. Cela signifie donc que tous les effets de violation de $C P$ sont 
reliés, d'où l'importance de surcontraindre le triangle d'unitarité par la mesure de tous ses paramètres. En effet, toute déviation pourrait être le signe de nouvelle physique. En pratique, il est utile d'utiliser la représentation du triangle d'unitarité dans le plan $(\bar{\rho}, \bar{\eta})$. On peut ramener les mesures à la détermination du sommet du triangle, de coordonnées $(\bar{\rho}, \bar{\eta})$, car le côté opposé est entièrement déterminé par les conventions mentionnées plus haut.

\subsubsection{Connaissances actuelles de la matrice CKM et du triangle d'unitarité}

\section{Mesure des éléments $V_{i j}$ de la matrice CKM}

Les différentes mesures des éléments $V_{i j}$ de la matrice CKM sont résumées dans le tableau 1.1.

\begin{tabular}{|c|c|}
\hline Moyenne mondiale de $V_{i j}$ & Méthode expérimentale \\
\hline \hline$\left|V_{u d}\right|=0,9738 \pm 0,0005$ & $\begin{array}{c}\text { Désintégration nucléaire } \beta \\
\text { (désintégration super-permise } \beta^{+}, \\
\text {désintégration } \beta^{-} \text {de neutrons polarisés, } \\
\left.\text { désintégration } \beta^{+} \text {de } \pi^{+}\right)\end{array}$ \\
\hline$\left|V_{u s}\right|=0,2200 \pm 0,0026$ & $\begin{array}{c}\text { Désintégration } K_{e 3}(K \rightarrow \pi l \nu), \\
\text { d'hyperons et de } \tau\end{array}$ \\
\hline$\left|V_{c d}\right|=0,224 \pm 0,012$ & $\begin{array}{c}\text { Production de di-muon par interaction } \\
\text { de } \nu \text { ou } \bar{\nu} \text { avec un noyau }\end{array}$ \\
\hline$\left|V_{c s}\right|=0,996 \pm 0,013$ & $\begin{array}{c}\text { Transition } D^{+} \rightarrow K^{0} l^{+} \nu \text { et } D^{0} \rightarrow K^{-} \nu \\
\text { et désintégration de bosons } W \text { réels }\end{array}$ \\
\hline$\left|V_{c b}\right|=(41,3 \pm 1,5) \times 10^{-3}$ & $\begin{array}{c}\text { Désintégrations semi-leptoniques inclusives } \\
\text { et exclusives des } B \\
(\text { transition } b \rightarrow c l \nu)\end{array}$ \\
\hline$\left|V_{u b}\right|=(3,67 \pm 0,47) \times 10^{-3}$ & $\begin{array}{c}\text { Désintégrations semi-leptoniques inclusives } \\
\text { et exclusives des } B \\
(\text { transition } b \rightarrow u l \nu)\end{array}$ \\
\hline$\left|V_{t b}\right|^{2} /\left(\left|V_{t d}\right|^{2}+\left|V_{t s}\right|^{2}+\left|V_{t b}\right|^{2}\right)=0,94_{-0,24}^{+0,31}$ & Désintégrations semi-leptoniques $t \rightarrow b l^{+} \nu$ \\
\hline
\end{tabular}

ТАВ. 1.1: Mesures actuelles des éléments de la matrice CKM [29].

\section{Mesure des angles du triangle d'unitarité}

1. $\beta$ est l'angle le mieux connu. Il a été mesuré avec une très grande précision, par les expériences BABAR et Belle, en utilisant les modes charmonium (avec la transition $b \rightarrow c \bar{c} s$ ), et en particulier la désintégration $B^{0} \rightarrow J / \psi K_{s}$. Ces canaux sont théoriquement très propres car ce sont des diagrammes en arbre qui dominent. Il existe en réalité une faible contribution en boucle, de l'ordre de $1 \%$, mais avec une même phase faible (à $\pi$ près). On mesure donc directement $\beta$. La valeur mondiale 
combinant les résultats de BABAR et BELLE est [29] :

$$
\sin (2 \beta)=0,736 \pm 0,049
$$

On peut également mesurer $\beta$ dans d'autres modes de désintégrations, mais il existe des contributions en boucles non négligeables dans ces derniers, qui peuvent faire intervenir des phases faibles différentes. Il est alors possible de mesurer une asymétrie de $C P$, mais il y a alors des incertitudes théoriques sur l'interprétation de l'asymétrie mesurée. On ne peut alors plus relier de façon propre la quantité mesurée à la phase $\beta$ et on mesure donc un $\beta$ effectif. En particulier, on utilise les transitions $b \rightarrow s$ pour ces mesures.

2. $\alpha$ peut être mesuré dans les modes non charmés (transition $b \rightarrow u \bar{u} d$ ) $: B^{0} \rightarrow$ $\pi \pi, \rho \pi, \rho \rho$. Ces modes étant pollués par des diagrammes en boucle, appelés pingouins, on mesure en réalité un $\alpha$ effectif. Il faut alors faire une analyse dans laquelle on suppose vérifiée la symétrie d'isospin pour extraire $\alpha$.

3. $\gamma$ : les différentes approches pour mesurer $\gamma$ sont exposées au chapitre 4 .

\section{Autres mesures permettant de contraindre le triangle d'unitarité}

Plusieurs autres mesures permettent également de contraindre la position du sommet du triangle d'unitarité, de coordonnées $(\bar{\rho}, \bar{\eta})$. Ces contributions sont résumées ci-dessous :

1. L'étude de la violation de $C P$ dans les kaons a permis de mesurer le paramètre de violation de $C P$ indirecte $\epsilon_{K}$, qui apporte des contraintes dans le plan $(\bar{\rho}, \bar{\eta})$.

2. Les mesures des fréquences d'oscillation des mésons $B_{d}\left(\Delta m_{d}\right)$ et des mésons $B_{s}$ $\left(\Delta m_{s}\right)$.

3. La mesure du rapport $\left|V_{u b} / V_{c b}\right|$ contraint la distance entre le sommet et le centre $(0,0): \sqrt{\bar{\rho}^{2}+\bar{\eta}^{2}}$.

L'effet de ces contraintes sur le plan $(\bar{\rho}, \bar{\eta})$ est montré sur la figure 1.4. L'ajustement de ces différentes contraintes conduit à $\bar{\rho}=0,189_{-0,070}^{+0,088}$ et $\bar{\eta}=0,358_{-0,042}^{+0,046}$ [30]. Les valeurs des angles $\alpha, \beta$ et $\gamma$ correspondantes sont résumées dans le tableau 1.2.

\begin{tabular}{|c|c|c|}
\hline angle $\left(\mathrm{en}^{\circ}\right)$ & valeur ajustée & valeur mesurée \\
\hline$\alpha$ & $94_{-10}^{+12}$ & $98_{-16}^{+15}$ \\
\hline$\beta$ & $23,8_{-2,0}^{+2,1}$ & $27,4_{-9,2}^{+1,9}$ \\
\hline$\gamma$ & $62_{-12}^{+10}$ & \\
\hline
\end{tabular}

TAB. 1.2: Valeurs des angles $\alpha, \beta$ et $\gamma$ du triangle d'unitarité. La première valeur est la valeur correspondant à l'ajustement global, et la deuxième valeur correspond à la valeur mesurée. 


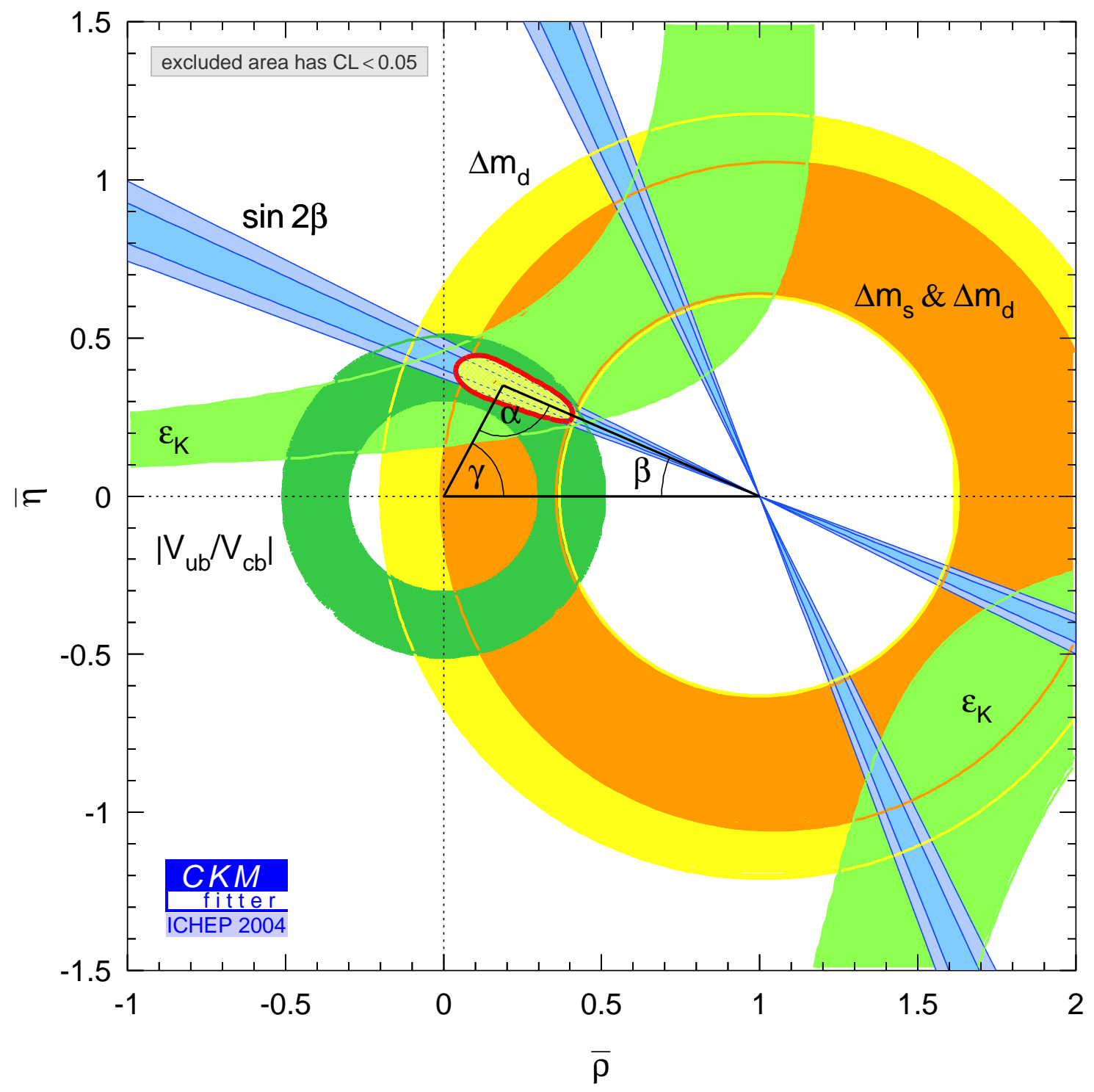

FIG. 1.4: Contraintes dans le plan $(\bar{\rho}, \bar{\eta})$ apportées par les mesures de $\epsilon_{K}, \Delta m_{d}, \Delta m_{s},\left|V_{u b} / V_{c b}\right|$ et $\sin (2 \beta)$. Le contour rouge montre le résultat de l'ajustement global à $95 \%$ de niveau de confiance. Cet ajustement a été réalisé par CKMFitter [30]. 
Dispositif expérimental 



\section{2}

\section{Le dispositif expérimental : BABAR et PEP-II}

\section{Introduction}

L'un des principaux buts de l'expérience BABAR [31,32] est l'étude systématique de l'asymétrie de $C P$ dans le système de mésons $B$. Les rapports d'embranchement des modes utilisés pour cette étude étant relativement faibles (inférieurs ou très inférieurs à $10^{-3}$ ), une très grande quantité de mésons $B$ est nécessaire. C'est pourquoi, le détecteur $B A B A R$ est situé sur un collisionneur $e^{+} e^{-}$(PEP-II) qualifié d'usine à $B$, qui fournit une luminosité très importante. La luminosité instantanée a atteint plus de $9,2.10^{33} \mathrm{~cm}^{-2} \mathrm{~s}^{-1}$ en mai 2004 . L'énergie dans le centre de masse est réglée à la résonance $\Upsilon(4 S)$ (état lié $b \bar{b}$ de masse $\left.10,58 \mathrm{GeV} / \mathrm{c}^{2}\right)$, ce qui permet de produire une importante quantité de paires $B \bar{B}$, avec un bon rapport signal/bruit $\left(\sigma_{b \bar{b}} / \sigma_{\text {tot }} \sim 0,28\right)$. Les mésons $B$ sont produits par le couplage de la paire $b \bar{b}$ avec des paires quarks/antiquarks provenant du vide $(u \bar{u}$ ou $d \bar{d})$. Deux types de mésons $B$ peuvent être produits : les neutres $B_{d}^{0} / \bar{B}_{d}^{0}$ et les chargés $B_{u}^{+} / B_{u}^{-}$. La masse de la résonance $\Upsilon(4 S)$ est légèrement supérieure à deux fois la masse d'un méson $B$, la paire $B \bar{B}$ est donc produite quasiment au repos dans le référentiel de l' $\Upsilon(4 S)$.

L'étude des mésons $B$ neutres est particulièrement intéressante, en particulier en ce qui concerne la violation de $C P$ dépendante du temps. Pour cela, il est nécessaire de connaître la saveur du méson $B$ considéré, ainsi que la différence de temps $\Delta t$ entre les désintégrations des deux $B$.

Le dispositif expérimental est particulièrement bien adapté à ce type de mesure. En effet, la paire $B \bar{B}$, qui constitue un système quantique antisymétrique, est produite de façon cohérente, ce qui signifie qu'avant la désintégration d'un des deux $B^{0}$, on a à tout instant un $B^{0}$ et un $\bar{B}^{0}$. Un des deux $B$ se désintègre dans l'état final que l'on veut étudier : on l'appelle $B_{\text {rec }}$. On peut étiqueter la saveur de l'autre $B$, appelé $B_{\text {tag }}$, grâce à ses produits de désintégration et en déduire la saveur de $B_{\text {rec }}$ au moment de la première désintégration.

Les mésons $B$ ayant une durée de vie très courte $\left(\tau_{B^{0}} \sim 1,536 p s\right)$ et étant produits quasiment au repos dans le référentiel de l' $\Upsilon(4 S)$, il est intéressant de leur conférer une quantité de mouvement importante dans le référentiel du laboratoire. Cela permet de bien 
séparer les points de désintégration des deux $B$. Pour cette raison, PEP-II a été conçu avec des faisceaux asymétriques en énergie, avec un facteur de Lorentz moyen $\langle\beta \gamma\rangle$ égal à 0,56 . La paire $B \bar{B}$ est émise principalement dans la direction des faisceaux, selon l'axe z. On peut donc affirmer avec une très bonne approximation que $\Delta t=\Delta z /(\beta \gamma c)$, où $\Delta z$ est la distance selon l'axe longitudinal $z$ du détecteur entre les désintégrations des deux $B . \Delta z$ est de l'ordre de $260 \mu m$, ce qui est mesurable par le détecteur de vertex de $B A B A R$.

\subsection{Le collisionneur PEP-II}

PEP-II (Positron-Electron Project) est un collisionneur électron/positron situé au SLAC (Stanford Linear Accelerator Center) en Californie [33].

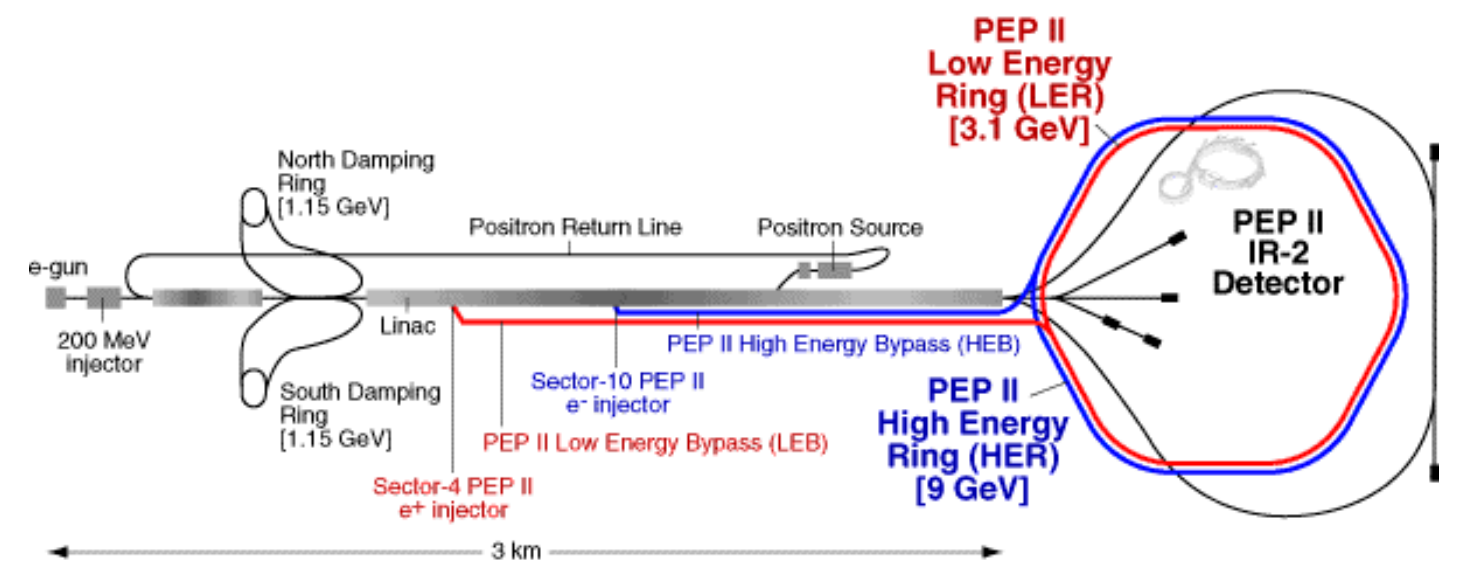

FIG. 2.1: Système d'injection de PEP-II : l'accélérateur linéaire (Linac) est représenté à gauche et le collisionneur à droite. L'IR2 est le point d'interaction des deux faisceaux, autour duquel se trouve le détecteur BABAR.

Les faisceaux utilisés sont asymétriques en énergie : le faisceau d'électrons est à $9 \mathrm{GeV}$, et celui de positrons à $3.1 \mathrm{GeV}$. L'énergie dans le centre de masse est de 10,58 GeV. Cela correspond à la résonance $\Upsilon(4 S)$, qui se désintègre quasiment à $100 \%$ en paires de $B \bar{B}$. Le bruit de fond principal, appelé continuum, est dû au processus $e^{+} e^{-} \rightarrow q \bar{q}(q=u, d, s, c)$. Pour l'étudier, on baisse l'énergie des faisceaux de $40 \mathrm{MeV}$ sous la résonance $\Upsilon(4 S)$. L'énergie est alors inférieure à deux fois la masse d'un méson $B$ et on ne produit donc plus de paire $B \bar{B}$. On peut alors étudier le continuum en l'absence de mésons $B$. Environ $10 \%$ des données sont prises à cette énergie.

PEP-II comporte deux parties principales (Fig. 2.1) : un accélérateur linéaire de $3 \mathrm{~km}$ qui accélère les particules jusqu'à leur énergie nominale et un anneau de stockage de 2,2 km de circonférence dans lequel a lieu la collision. Au début de l'accélérateur linéaire, un canon à électrons produit les électrons par effet thermoélectrique. Une partie de ces électrons est déviée, après avoir été accélérés, vers une cible fixe de tungstène pour produire le faisceau de positrons, qui est ensuite ramené au début de l'accélérateur linéaire grâce à la ligne de retour des positrons. Les électrons et les positrons sont d'abord accélérés dans 
l'accélérateur linéaire pour atteindre leur énergie nominale et sont ensuite conduits dans leur anneau de stockage.

L'accélérateur linéaire est également utilisé pour d'autres expériences et peut produire des faisceaux allant jusqu'à $50 \mathrm{GeV}$. Les électrons et les positrons n'utilisent donc qu'une partie de cet accélérateur. Comme les faisceaux sont asymétriques en énergie, l'anneau de stockage comporte deux sous-anneaux avec des champs magnétiques de différentes intensités : le $H E R$ (High Energy Ring) pour les électrons avec une intensité de courant de 2.14 A et le $L E R$ (Low Energy Ring) pour les positrons avec une intensité de courant de 0.75 A. Le HER correspond à l'ancien anneau de PEP-I, alors que le $L E R$ a été construit pour l'expérience BABAR.

Au démarrage en 1999, les $e^{+}$et les $e^{-}$étaient injectés dans l'anneau de stockage par paquets d'environ $10^{9}$ particules, à une fréquence comprise entre 1 et $30 \mathrm{~Hz}$. En moyenne, les paquets étaient espacés de 4 ns. En fonctionnement normal, l'injection se faisait environ toutes les 40 à 50 minutes. L'injection, introduisait du temps mort car il fallait un certain temps pour avoir les deux faisceaux à la fois, avec l'intensité de courant désirée. Il fallait de plus baisser les tensions des différents sous-détecteurs, puis les remettre sous tension. En conséquence, la luminosité moyenne utile n'était que 70 à $75 \%$ de la luminosité disponible. Une nouvelle stratégie a donc été mise en place au début de l'année 2004 pour augmenter la luminosité. Cela consiste à injecter des particules en continu dans les anneaux de stockage, sans avoir à baisser les tensions des composants de BABAR. Dans un premier temps, ce système d'injection continu ne se faisait que sur l'anneau de stockage du faisceau de basse énergie. Ce choix a été guidé par le fait que la durée de vie de ce faisceau est plus faible. De plus, il se trouve qu'historiquement, il y avait moins de problèmes de bruits de fond lors de l'injection de ce faisceau. Le gain a été d'environ $35 \%$ et la machine est devenue plus fiable. Ce système s'est avéré être très performant et le gain est en réalité plus de deux fois supérieur à celui attendu. Cela s'explique par le fait que le régime de la machine est plus stable avec ce système. Ensuite, ce système d'injection continue a été mis en place dans les deux faisceaux. Il en résulte un gain supplémentaire de $12 \%$, mais sans amélioration signification de la stabilité. Une des principales préoccupations est de fournir un courant suffisant pour ne pas perdre les faisceaux, sans délivrer de radiation excessive dans le détecteur.

Les faisceaux se rencontrent en position frontale au centre du détecteur BABAR. Ce choix d'une technique bien maîtrisée, rend en revanche la séparation des faisceaux relativement difficile après le point d'interaction. En réalité, l'axe de collision est décalé de l'axe z d'environ 20 mrad dans le plan horizontal, et ce afin de minimiser l'effet du champ magnétique produit par le solenoïde de BABAR sur les faisceaux dans la région d'interaction. Il faut donc tourner les faisceaux très rapidement juste avant leur collision et les séparer juste après afin d'éviter des collisions parasites. Cela est compliqué par le très grand nombre de paquets de particules dans les anneaux (environ 1000) : en effet, les paquets ne sont séparés que d'environ $1,2 \mathrm{~m}$.

La région d'interaction est décrite par la figure 2.2. La focalisation se fait grâce à une série de quadripôles $\left(Q_{1}, Q_{2}, Q_{4}\right.$ et $Q_{5}$ sur la figure) et la séparation grâce à des dipôles d'un champ moyen de $0,84 \mathrm{~T}$, situés à $21 \mathrm{~cm}$ du point d'interaction ( $B_{1}$ sur la figure). A noter que les aimants $B_{1}$ et $Q_{1}$ sont situés à l'intérieur du détecteur. Des masques sont placés à l'intérieur de ces aimants pour limiter le rayonnement synchrotron. Afin d'avoir 
un angle solide le plus grand possible pour le détecteur, il est nécessaire que ces éléments de PEP-II soient très compacts. De plus, le tube à vide doit être le plus fin possible pour que le premier sous-détecteur (le détecteur de vertex) soit le plus près possible du point d'interaction. Le rayon du tube à vide est de $2,5 \mathrm{~cm}$. L'ensemble comprenant les aimants $\mathrm{Q} 1$ et $\mathrm{B} 1$, le tube à vide et le détecteur de vertex est maintenu par un tube rigide de 43 $\mathrm{cm}$ de diamètre.

Il est primordial d'avoir un bon vide dans la machine afin de minimiser le bruit de fond et de limiter le niveau de radiation qui risquerait d'endommager le détecteur. Des collimateurs et des pompes à vide atténuent le bruit de fond machine, qui est dû à l'interaction des faisceaux avec le gaz résiduel dans le tube à vide dans lequel a lieu la collision.

\section{PEP-II Interaction Region}

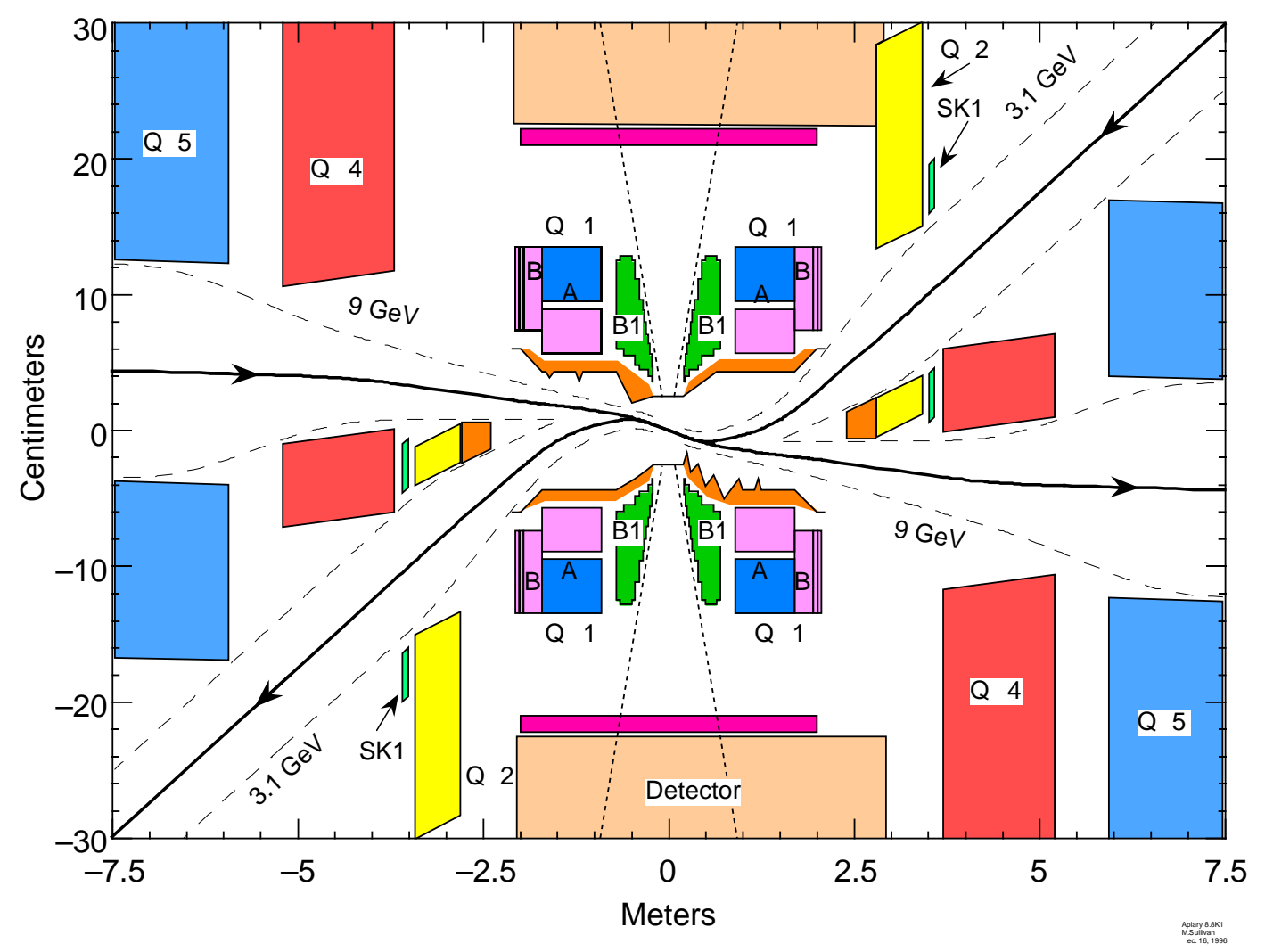

FIG. 2.2: Système optique de PEP-II au voisinage du point d'interaction, avec une échelle dilatée selon l'axe verticale.

La luminosité absolue de PEP-II est déterminée grâce à des processus QED de type production de paires $e^{+} e^{-}$ou $\mu^{+} \mu^{-}$et les énergies moyennes des faisceaux sont calculées à partir de toutes les forces magnétiques mises en jeu et de la déviation moyenne des fréquences accélératrices par rapport à leur valeur centrale. 


\section{$2.2 \quad$ Le détecteur BABAR}

Le but premier de BABAR étant l'étude systématique de la violation de CP dans le système de mésons $B$ neutres, le détecteur a été optimisé pour ce type de mesures. Il permet cependant l'étude d'autres sujets de physique, tels que les mesures de précision des désintégrations des mésons beaux ou charmés et des leptons $\tau$, ainsi que les recherches de processus rares. Le détecteur BABAR a été conçu de façon à avoir une acceptance optimale en particulier dans la direction dans laquelle les mésons $B$ sont émis, ce qui a conduit à un détecteur asymétrique. L'acceptance géométrique de BABAR est proche de $4 \pi$. La mesure de la distance entre les vertex des deux $B$ étant primordiale, il est nécessaire d'avoir une excellente résolution près du point d'interaction. Le détecteur doit également être capable de mesurer des traces avec des impulsions comprises entre $60 \mathrm{MeV} / c$ et $5 \mathrm{GeV} / c$ et de bien discriminer les électrons, les muons, les pions, les kaons et les protons. La limite supérieure en impulsion correspond à l'impulsion maximale permise par la cinématique, alors que la limite inférieure provient du détecteur qui n'est pas parfait. Enfin, le détecteur doit pouvoir détecter les photons et les $\pi^{0}$ dans un domaine d'énergie allant de $20 \mathrm{MeV}$ à $5 \mathrm{GeV}$. C'est dans le but de répondre à toutes ces exigences que le détecteur BABAR a été réalisé.

La figure 2.3 représente une vue longitudinale et une vue transverse du détecteur.

BABAR a une structure classique en oignon et est composé de plusieurs sous-détecteurs qui sont, en partant du point d'interaction des faisceaux :

- Un détecteur de vertex (SVT), qui permet la mesure des vertex proches du point d'interaction des faisceaux et la détermination des traces chargées de faible impulsion transverse $\left(p_{t}<120 \mathrm{MeV} / c^{2}\right)$.

- Une chambre à dérive pour déterminer l'impulsion des traces chargées, courbées par le champ magnétique du solénoïde. Elle sert également à l'identification des particules par la mesure de $d E / d x$.

- Le DIRC (Detector of Internally Reflected Cherenkov Light) qui utilise le rayonnement Cherenkov pour identifier les hadrons chargés $(\pi, \mathrm{K}, \mathrm{p})$.

- Un calorimètre électromagnétique pour détecter les photons et les hadrons neutres et identifier les électrons.

- Un aimant supraconducteur de 1,5 T.

- Un retour de champ instrumenté (IFR) pour l'identification des muons et la mesure de l'énergie des hadrons neutres lourds.

\subsubsection{Le détecteur de vertex (SVT)}

La tâche principale du SVT [34,35] est la reconstruction du vertex des deux mésons $B$ produits par la résonance $\Upsilon(4 S)$ avec une excellente résolution dans la direction longitudinale. Afin d'étudier l'asymétrie de $C P$ dépendante du temps, il est indispensable de mesurer avec précision la distance entre les désintégrations des deux $B$, qui est en moyenne de $260 \mu \mathrm{m}$. L'asymétrie des faisceaux fait que les $B$ sont émis selon l'axe longitudinal $z$. Une excellente résolution selon l'axe $z$ est donc nécessaire. Cela est rendu possible par l'utilisation de détecteurs de silicium à micro-pistes. La résolution d'un vertex de $B$ totalement reconstruit est meilleure que $80 \mu \mathrm{m}$ selon l'axe $z$. La résolution dans le plan 


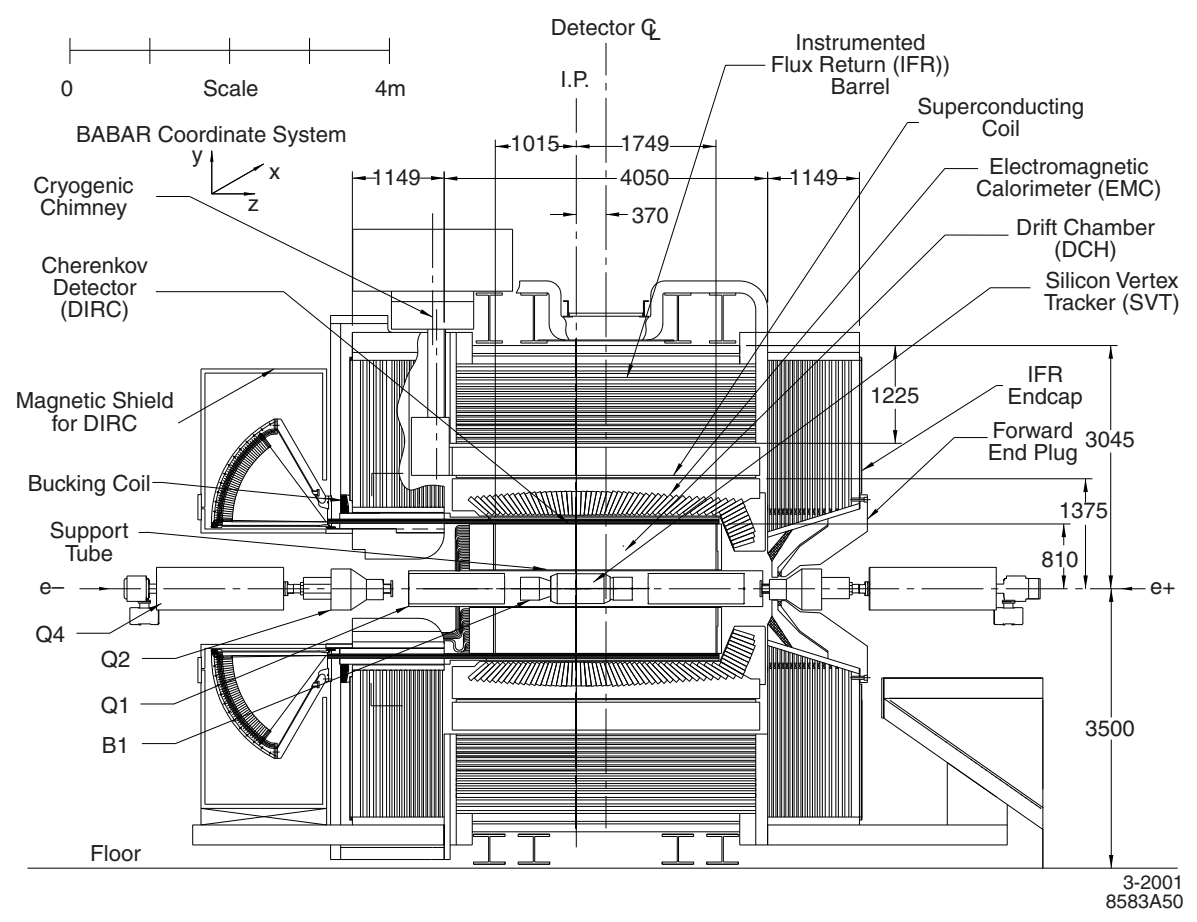

(a) Section longitudinale

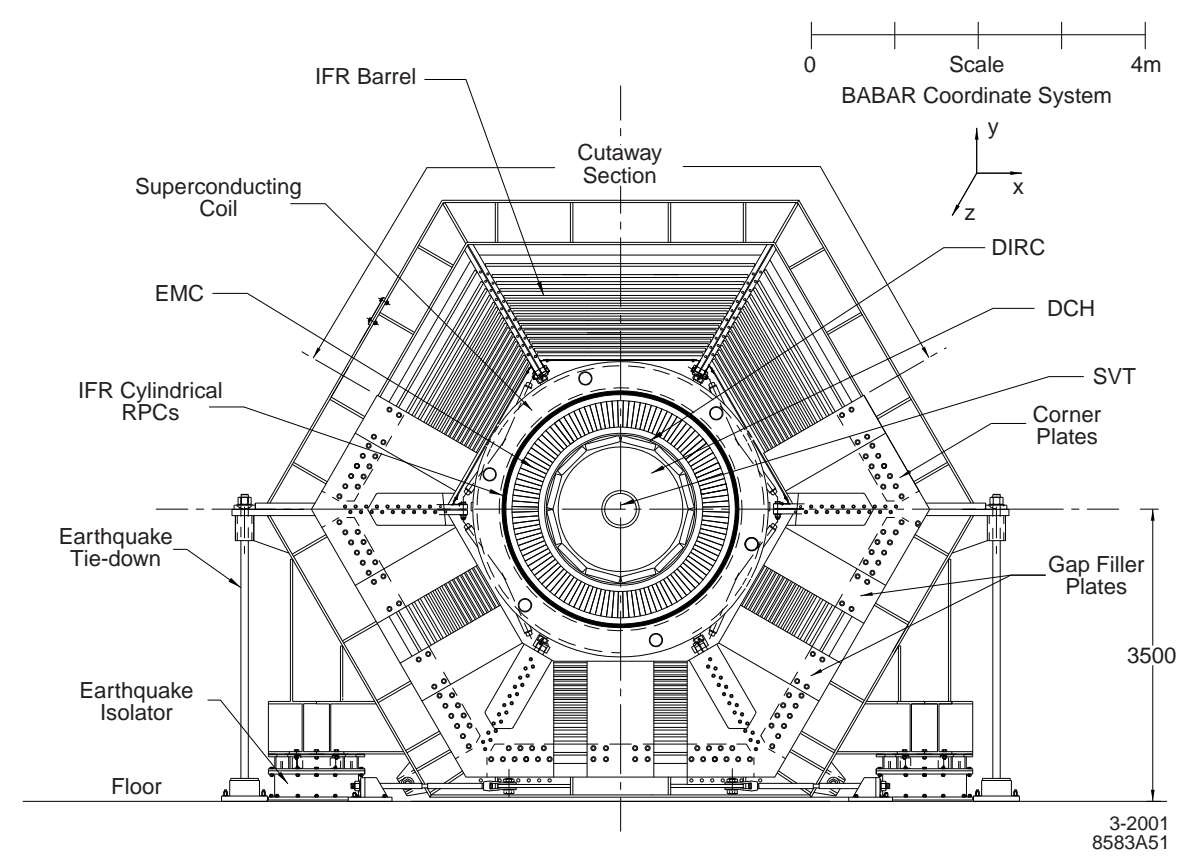

(b) Section transverse

Fig. 2.3: Architecture de BABAR. 
transverse $x-y$ doit également être performante afin de reconstruire les états finals de désintégration des $B$, mais aussi des désintégrations charmées et du $\tau$.

De plus, le SVT contribue de façon importante à la mesure de l'impulsion des traces chargées. Pour les particules énergétiques, cette mesure est complétée par les mesures dans la chambre à dérive, mais pour les particules dont l'impulsion transverse est inférieure à $120 \mathrm{MeV} / c^{2}$, seul le SVT peut être utilisé, car la courbure de leur trajectoire sous l'effet du champ magnétique ne leur permet pas de traverser un nombre suffisant de couches dans la chambre à fils. Le SVT contribue en particulier de façon importante à la reconstruction des pions peu énergétiques $\pi^{ \pm}$issus de la désintégration du $D^{* \pm}$. Ce détecteur est donc très important pour l'analyse de la violation de $C P$ dans les désintégrations $B \rightarrow D^{* \mp} \pi^{ \pm}$. Le SVT est capable de reconstruire la trajectoire des particules ayant une impulsion comprise entre 50 et $120 \mathrm{MeV} / \mathrm{c}^{2}$ avec une efficacité de $70 \%$. D'autre part, la contribution du SVT est primordiale, car pour une trace donnée, la meilleure information angulaire est donnée par les points proches du vertex. Le SVT participe également à l'identification des particules par la mesure de leur perte d'énergie par ionisation $(d E / d x)$, qui suit la loi de Bethe-Bloch.

La conception du SVT a été guidée par de nombreuses exigences en termes d'efficacité et par de nombreuses contraintes. Le choix du nombre de couches s'est révélé être un compromis entre l'efficacité intrinsèque du SVT seul et l'efficacité du SVT associé à la chambre à fils. La localisation particulière du SVT, qui est solidaire du tube à vide, le tout étant placé dans un cylindre rigide, a imposé certaines contraintes sur la construction du SVT : il s'agissait d'avoir une couverture angulaire maximale, malgré l'encombrement dû aux aimants placés à l'intérieur du détecteur. Il fallait de plus un détecteur fin afin de ne pas trop détériorer l'énergie des particules. Enfin, il était nécessaire que le SVT soit extrêmement résistant aux radiations, car ce sous-détecteur est placé très près des faisceaux. De plus, il doit être fiable et solide car sa localisation peu facile d'accès rend toute intervention sur le SVT très longue. Toutes ces raisons ont conduit à construire un détecteur de vertex constitué de 5 couches cylindriques concentriques de détecteurs double face en silicium, de $30 \mu \mathrm{m}$ d'épaisseur. Chacune de ces couches est composée de modules. La structure en couches du SVT est schématisée par les Fig. 2.4 et 2.5. Cette géométrie a été choisie afin de minimiser la quantité de silicium utilisée tout en ayant une couverture angulaire maximum.

Les trois couches internes, situées entre 32 et $54 \mathrm{~mm}$ de distance des faisceaux comportent chacune six modules. Ces couches sont principalement dédiées à la mesure du paramètre d'impact, qui est le point de plus proche approche entre la trajectoire d'une particule chargée et l'axe des faisceaux. La résolution de ces couches est comprise entre 10 et 15 microns. Les deux couches externes sont quant à elles situées à une distance comprise entre 91 et $144 \mathrm{~mm}$ et constituées respectivement de 16 et 18 modules. Elles permettent les mesures angulaires et la reconstruction des traces. Afin de minimiser la quantité de silicium utilisée, elles ont une structure en arche. Chacune de ces couches externes est constituée de deux sous-couches a et b (Fig. 2.5) situées à des distances radiales légèrement différentes. Cela permet d'augmenter la couverture en angle solide. La résolution des couches externes est de l'ordre de $40 \mu \mathrm{m}$.

Les faces internes du détecteur comportent des pistes perpendiculaires à la direction des faisceaux, ce qui permet de mesurer la position longitudinale selon l'axe z, alors que 


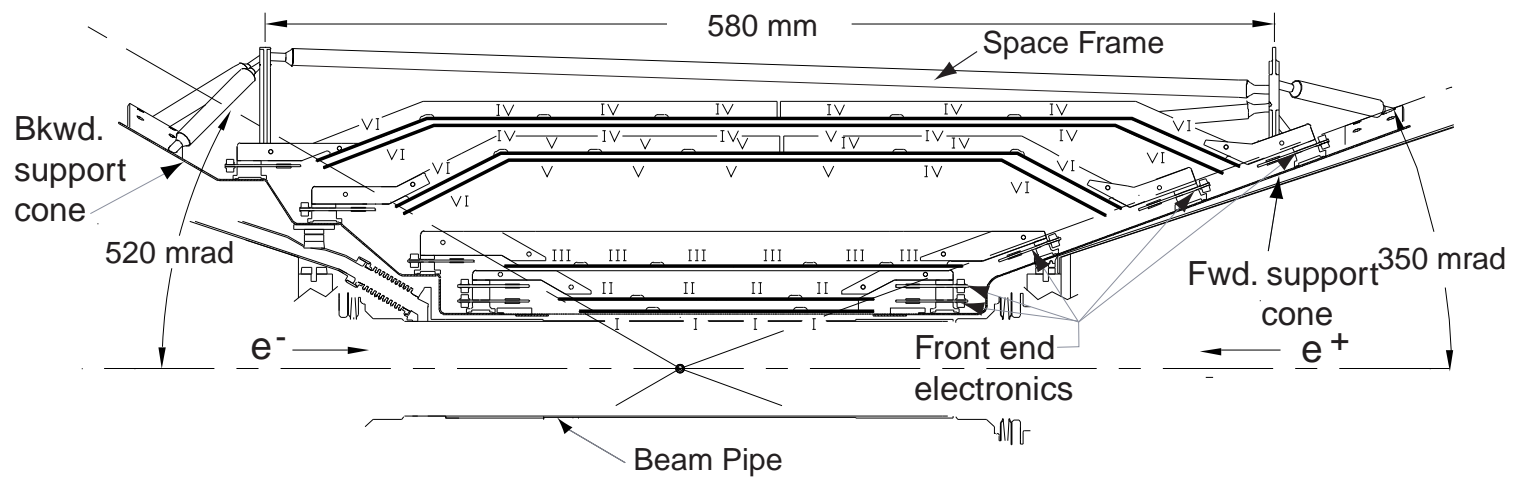

FIG. 2.4: Section longitudinale de l'architecture du SVT.

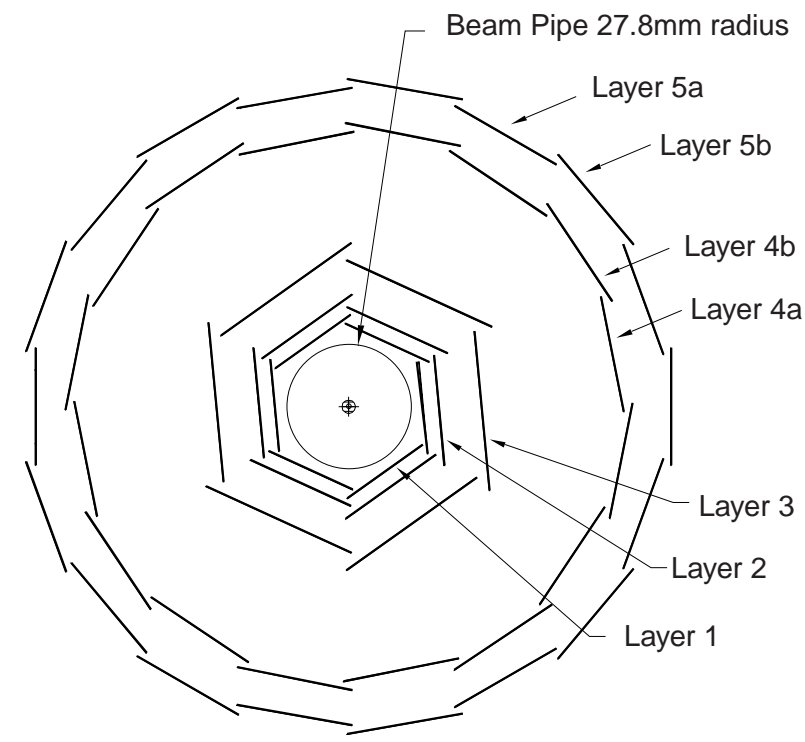

FIG. 2.5: Section transverse de l'architecture du SVT. 
les pistes des faces externes sont parallèles à l'axe des faisceaux et permettent la mesure de l'angle azimutal $\phi$. Le SVT est constitué en tout de 340 détecteurs de silicium, ce qui correspond à une aire de $1 \mathrm{~m}^{2}$. Environ 150000 canaux de lecture sont utilisés. Afin de minimiser la présence de matériel dans la région d'acceptance, l'électronique de lecture est entièrement située en dehors du volume actif du détecteur. L'acceptance angulaire du SVT est très bonne, car il couvre des angles polaires compris entre $20^{\circ}$ et $150^{\circ}$ dans le référentiel du laboratoire, ce qui correspond à une couverture de $90 \%$ d'angle solide dans le référentiel du centre de masse. L'efficacité moyenne d'un module du SVT, obtenue en comptant les traces détectées et les traces traversant le détecteur, est de 97 \% (Fig. 2.6). La résolution sur la mesure de la perte d'énergie d'une particule par unité de longueur $(d E / d x)$ est de $14 \%$, ce qui permet une séparation kaon/pion de plus de $2 \sigma$ pour des impulsions inférieures à $500 \mathrm{MeV}$. Pour les trace énergétiques $\left(p_{t}>3 \mathrm{GeV} / c\right)$, la résolution longitudinale est de $29 \mu \mathrm{m}$ et la résolution azimutale de 0,43 $\mathrm{mrad}$.

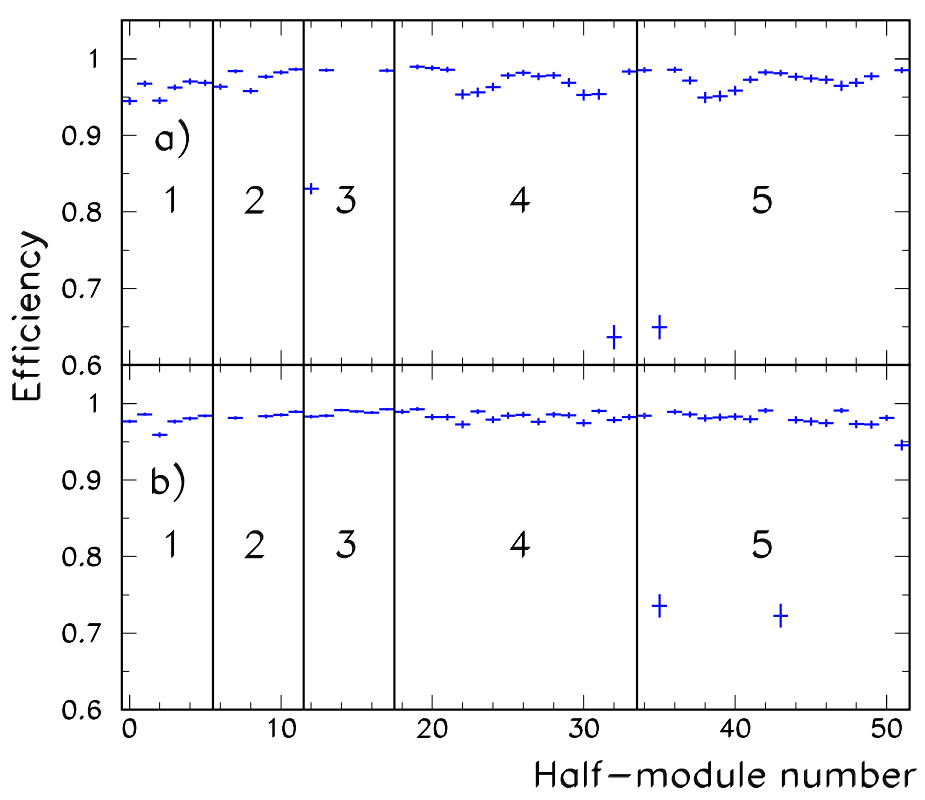

FIG. 2.6: Efficacité de détecter les coordonnées $\phi$ et $z$ pour des traces traversant les demi modules avant (a) et arrière (b), en fonction du numéro de couche et du numéro de module.

Les différents modules du SVT ont été assemblés avec une précision de l'ordre de $200 \mu \mathrm{m}$. Il est donc nécessaire d'aligner le SVT. Le SVT est mécaniquement lié à PEPII et non à $B A B A R$. Cela implique un alignement en deux étapes. Il faut d'abord faire l'alignement interne qui consiste à déterminer la position relative de chaque module par rapport à ses voisins. Cet alignement est stable au cours du temps, tant qu'on ne touche pas directement au collisionneur ou au SVT. Il faut ensuite aligner le SVT par rapport au reste du détecteur, en prenant comme référence la chambre à dérive. Cet alignement peut se modifier à l'échelle d'une journée, en raison par exemple de changements de température. Il est donc recalculé à chaque run, c'est-à-dire toutes les 2-3 heures. 


\subsubsection{La chambre à dérive $(\mathrm{DCH})$}

La chambre à dérive $[36,37]$ sert principalement à détecter les particules chargées et à mesurer précisément leur impulsion et leurs angles. Une haute précision est nécessaire afin de permettre la reconstruction exclusive des mésons $B$ et $\mathrm{D}$ avec un minimum de bruit de fond. Elle permet d'avoir jusqu'à 40 points de mesure pour reconstruire une trace. Combinées avec l'information du SVT, les mesures de la chambre à dérive ont une très haute efficacité de reconstruction pour les traces ayant une impulsion transverse supérieure à $100 \mathrm{MeV} / c$. La chambre à dérive doit également être capable de reconstruire les vertex de désintégration et d'interaction qui ne sont pas situés dans le SVT, comme par exemple la désintégration des $K_{S}^{0}$. C'est pourquoi, il est important d'avoir une bonne résolution longitudinale (de l'ordre de $1 \mathrm{~mm}$ ).

La chambre à dérive est utilisée pour l'identification des particules de basse impulsion par la mesure de $d E / d x$, avec une résolution de $7 \%$. Elle permet de séparer les pions des kaons jusqu'à $700 \mathrm{MeV} / \mathrm{c}^{2}$, apportant ainsi une information complémentaire de celle du DIRC (section 2.2.3), en énergie et en acceptance angulaire (Fig. 2.7). Enfin, la chambre à dérive participe au système de déclenchement.

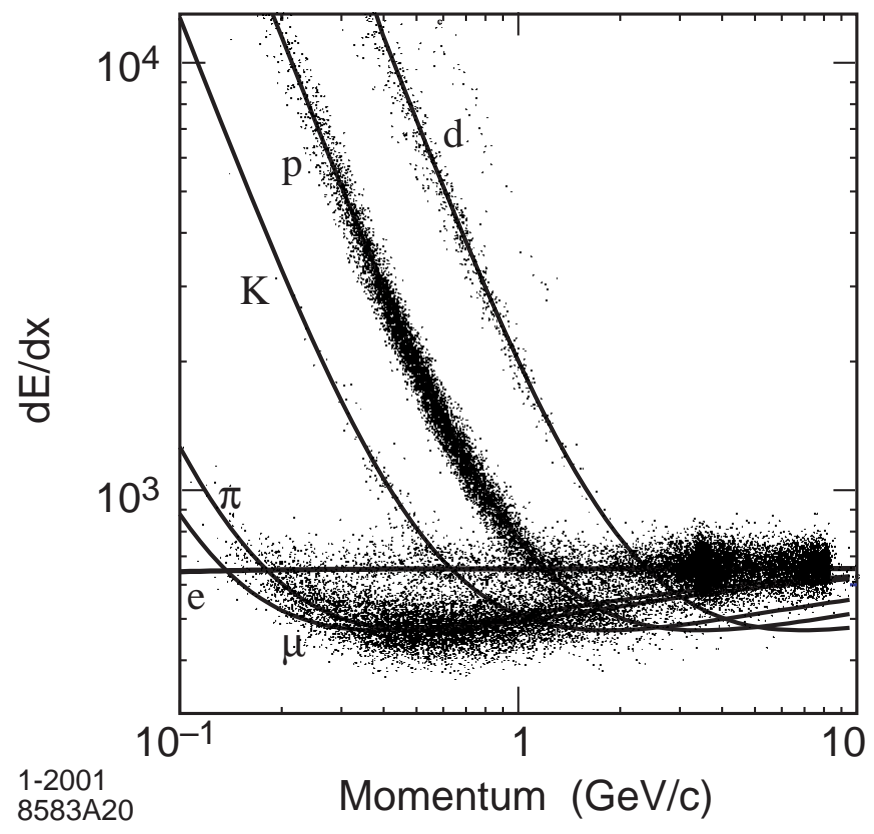

Fig. 2.7: Mesure de $d E / d x$, la perte d'énergie par unité de longueur dans la chambre à dérive, en fonction de l'impulsion des traces détectées, pour divers échantillons.

La chambre à dérive est conçue de façon à minimiser la présence de matière, en particulier vers l'avant. En effet, l'impulsion moyenne des produits de désintégration des $B$ et des $D$ étant de l'ordre de quelques centaines de $\mathrm{MeV} / \mathrm{c}$, la mesure est principalement limitée par la diffusion multiple des particules. Cela impose certaines contraintes sur le choix des matériaux utilisés.

La chambre consiste en un cylindre de $280 \mathrm{~cm}$ de long, avec un rayon interne de 23,6 $\mathrm{cm}$ et un rayon externe de $80,9 \mathrm{~cm}$. Le cylindre interne est constitué d'une couche de 
beryllium de $1 \mathrm{~mm}$ d'épaisseur et le cylindre externe consiste en deux couches de fibres de carbone. Les parois des matériaux mis en jeux sont suffisamment fines pour ne pas détériorer la précision de la chambre et des autres détecteurs. L'épaisseur totale de la chambre est de $1,08 X_{0}$, où $X_{0}$ représente la longueur de radiation. La chambre à fils est remplie d'un mélange gazeux Helium-Isobutane dans les proportions 80\%: $20 \%$. Ce choix permet d'avoir une bonne résolution spatiale et une bonne résolution sur la mesure de $d E / d x$, tout en ayant un temps de dérive relativement court.

La chambre comporte 7104 cellules de dérive hexagonales de dimension typique de 11,9 mm sur 19,0 mm. Ces cellules sont assemblées en 10 super-couches de 4 couches chacune, ce qui permet d'avoir jusqu'à 40 points de mesure par trace. Afin de pouvoir faire une mesure en trois dimensions, l'angle stereo des super-couches alterne entre une configuration axiale $(\mathrm{A})$ et stereo $(\mathrm{U}, \mathrm{V})$ avec des angles compris entre $\pm 45 \mathrm{mrad}$ et \pm $76 \mathrm{mrad}$, comme le montre la Fig. 2.8. Les couches axiales mesurent l'angle de courbure des traces, afin de déterminer leur impulsion, alors que les couches stereo mesurent aussi la position longitudinale des traces. L'acceptance angulaire de la chambre est comprise entre $17,2^{\circ}$ et $152,6^{\circ}$ dans le référentiel du laboratoire.

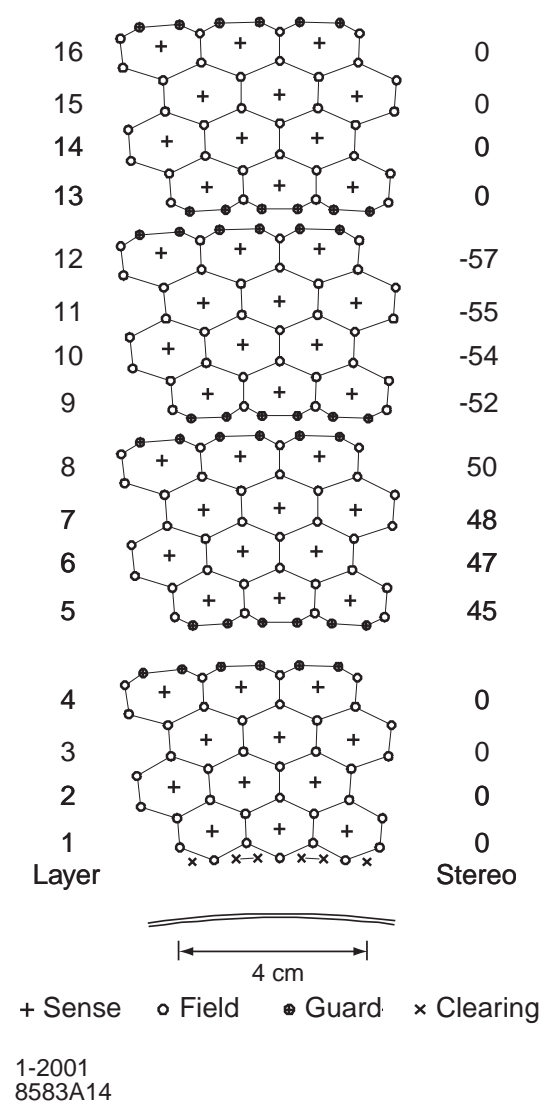

Fig. 2.8: Vue transverse de la disposition de quelques cellules de détection de la chambre à dérive. Des lignes ont été ajoutées entre les fils de champ pour aider à la visualisation des limites des cellules. Les chiffres sur la droite donnent les angles stereo (en mrad) des fils capteurs de chaque couche.

Chaque cellule est constituée d'un fil capteur entouré de six fils de champ (Fig. 2.8). 
Les fils capteurs, de $20 \mu m$ d'épaisseur, sont en alliage tungstène-rhenium plaqué or et possèdent donc à la fois une faible résistivité et une bonne résistance mécanique. Ils sont soumis à une tension d'environ $1900 \mathrm{~V}$. Les fils de champ, d'épaisseur comprise entre 80 et $120 \mu \mathrm{m}$, sont en aluminium plaqué or. Les fils de champ qui séparent les super-couches ont une tension de $340 \mathrm{~V}$ afin d'éviter la déformation des lignes de champ aux limites des super-couches, alors que les autres sont à la masse. Il y a en tout 28768 fils dans la chambre.

Le principe de mesure est celui d'une chambre à fils classique. Une particules chargée ionise le gaz en le traversant. Les électrons arrachés sont accélérés et migrent vers les fils capteur, déclenchant ainsi une avalanche électromagnétique qui est détectée par le fil. Le gain d'avalanche est de l'ordre de grandeur de 5.104. Les signaux sont enregistrés par un système électronique qui mesure le temps de dérive des électrons et la charge déposée. Le seuil de détection de la chambre est de 2 électrons primaires, sachant qu'une trace chargée en produit en moyenne 22. La haute luminosité de PEP-II impose un temps mort très court pour la chambre à dérive et le système de lecture. La résolution temporelle de l'électronique de la chambre est de $1 \mathrm{~ns}$. La figure 2.9 représente une modélisation de la dérive des électrons dans le gaz pour une cellule hexagonale. La mesure du temps de dérive permet de connaître la position de la trace détectée pour une cellule individuelle avec une précision de $140 \mu \mathrm{m}$. La résolution sur l'impulsion transverse est modélisée par :

$$
\frac{\sigma_{p_{t}}}{p_{t}}=(0,13 \pm 0,01) \% p_{t}(\mathrm{GeV} / c)+(0,45 \pm 0,03) \%
$$

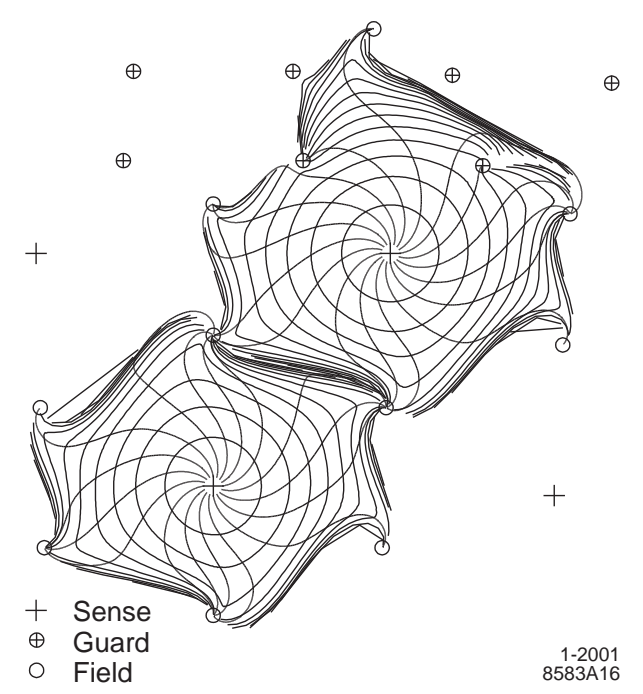

FIG. 2.9: Isochrones (contour des temps de dérive égaux des ions) espacées de $100 \mathrm{~ns}$ des cellules de la chambre à dérive.

L'efficacité de la chambre à dérive est de $98 \%$ à la tension nominale de $1960 \mathrm{~V}$. Si on diminue cette tension à $1900 \mathrm{~V}$, on observe une légère perte d'efficacité (Fig. 2.10). 

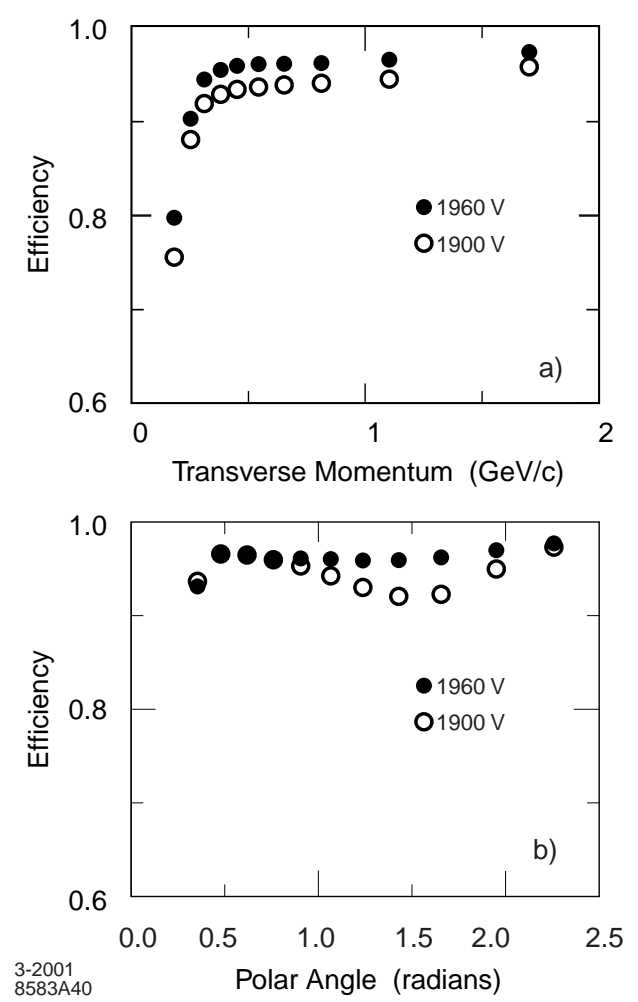

Fig. 2.10: Efficacité de reconstruction de la chambre à dérive pour les deux tensions de fonctionnement de $1900 \mathrm{~V}$ et $1960 \mathrm{~V}$, en fonction de l'angle polaire et de l'impulsion. 


\subsubsection{Le DIRC (Detection of Internally Reflected Cherenkov light)}

Le DIRC $[38,39]$ est un détecteur original basé sur le rayonnement Cherenkov qui sert à l'identification des particules chargées et en particulier des kaons. L'identification des K produits par la cascade $b \rightarrow c \rightarrow s$ avec des impulsions allant jusqu'à $2 \mathrm{GeV} / c$ est en effet primordiale pour l'étiquetage de la saveur des B. Il est d'autre part nécessaire de pouvoir distinguer les $\mathrm{K}$ des $\pi$ à haute impulsion (jusqu'à $4 \mathrm{GeV} / \mathrm{c}$ ) avec de petits angles polaires dans le référentiel du laboratoire, pour l'étude de certaines désintégrations rares des $B$, comme $B \rightarrow \pi^{+} \pi^{-} / K^{+} \pi^{-}$.

Le principe du DIRC consiste à utiliser l'information des photons émis par rayonnement Cherenkov par une particule chargée traversant un milieu radiateur d'indice $n$ avec une vitesse $\beta>1 / n$. Les photons sont émis dans un cône, d'angle d'ouverture $\theta_{C}$ autour de la trace de la particule. Le but du DIRC est de mesurer cet angle, appelé angle de Cherenkov et d'en déduire l'identité de la particule chargée. L'angle $\theta_{C}$ est directement relié à la vitesse de la particule, par la relation :

$$
\cos \theta_{C}=\frac{1}{n \beta}
$$

Connaissant l'impulsion de la particule à l'entrée du DIRC, donnée par la DCH et le SVT, on peut en déduire la masse de la particule et ainsi l'identifier, en utilisant la relation :

$$
m^{2} c^{2}=\frac{1-\beta^{2}}{\beta^{2}} p^{2}
$$

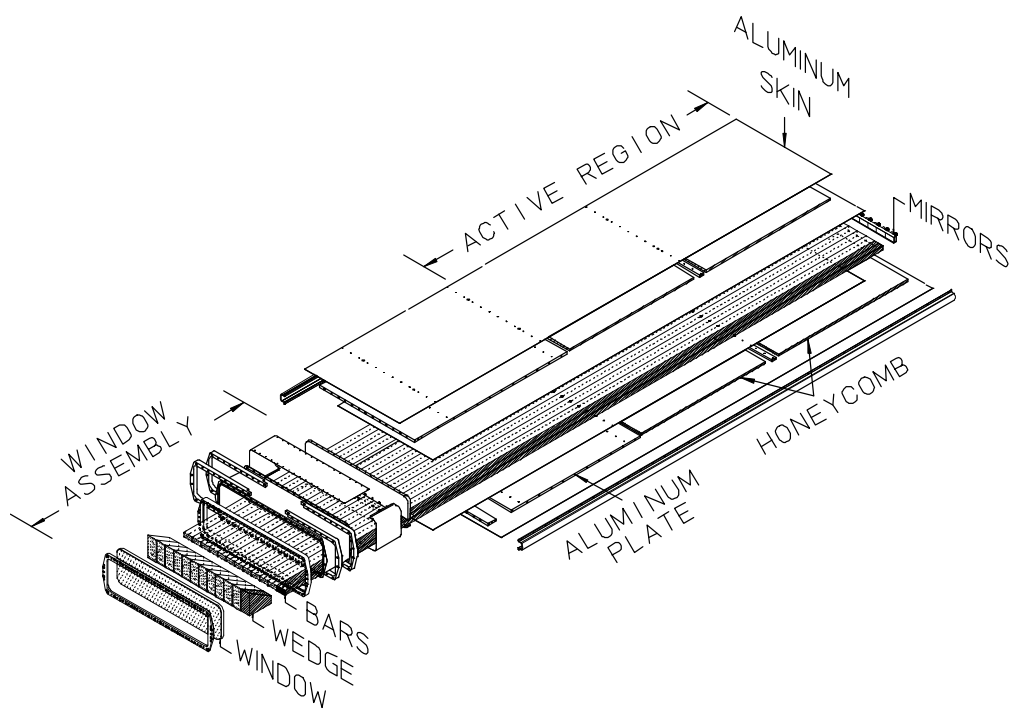

FIG. 2.11: Une boîte de barres de quartz du DIRC.

Le milieu radiateur du DIRC [40] est composé de 144 barres de quartz rangées dans 12 secteurs, arrangés de façon à former un polygone régulier centré sur l'axe du faisceau. Une 
partie des photons de Cherenkov émis par une particule chargée traversant le milieu radiateur est piégée dans la barre par réflexion totale. Chaque photon subit alors de multiples réflexions internes dans la barre et est détecté à sa sortie. Le principe de fonctionnement du DIRC est schématisé Fig. 2.12. La Fig. 2.13 représente la géométrie du DIRC. Afin de minimiser la quantité de détecteur, un miroir est placé à une des extrémités de la barre pour réfléchir les photons vers l'autre extrémité, où se trouve la partie instrumentée. Celle-ci est située vers l'arrière pour ne pas détériorer la mesure des particules, car l'asymétrie du collisionneur favorise l'émission de particules vers l'avant. La mesure repose sur le principe que l'angle de Cherenkov est conservé le long de ses réflexions, modulo un certain nombre d'ambiguïtés. Ces ambiguïtés sont dues au fait que l'on ne connait pas la parité du nombre de réflexions dans la barre, et que l'on ne sait pas non plus si le photon a été réfléchi par le miroir, s'il est sorti par la gauche ou par la droite de la barre.

Le quartz est un matériau particulièrement bien adapté, car il est résistant aux radiations d'ionisation, il possède une grande longueur d'atténuation, son indice de réfraction est grand $(\mathrm{n}=1,474)$ et sa dispersion chromatique dans le domaine des longueurs d'onde mises en jeu (du visible à l'ultra-violet proche) est faible. Chaque barre comprend 4 parties collées pour former une barre d'une longueur de 4,9 m. La section transverse de ces barres est de 1,7 cm d'épaisseur et de 3,5 cm de large. Elles sont situées à environ $810 \mathrm{~mm} \mathrm{du}$ point d'interaction. Les barres sont isolées optiquement les unes des autres par une fente d'air de $150 \mu \mathrm{m}$ d'épaisseur.

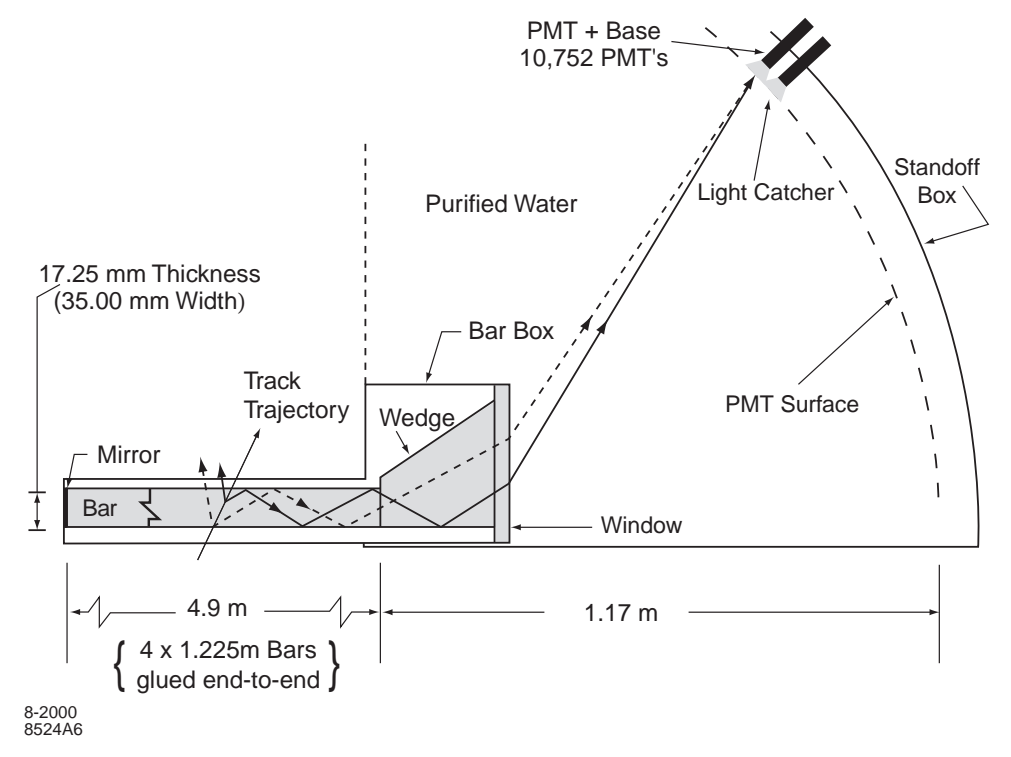

FIG. 2.12: Principe de fonctionnement du DIRC.

Les barres sont plongées dans un flux d'azote, qui a un indice de réfraction suffisamment différent du quartz pour rendre les surfaces bien réfléchissantes. De plus, ce gaz évite la formation d'humidité par condensation à la surface des barres. On mesure l'humidité à la sortie du flux d'azote pour détecter d'éventuelles fuites d'eau. Au bout de chaque barre, se trouve un prisme en quartz de $91 \mathrm{~mm}$ de long et de section trapezoïdale de bases de $29 \mathrm{~mm}$ et $79 \mathrm{~mm}$. L'intérêt du prisme est de rabattre les photons ayant un trop grand angle (vers le haut ou vers le bas) vers des directions plus longitudinales. Cela permet 


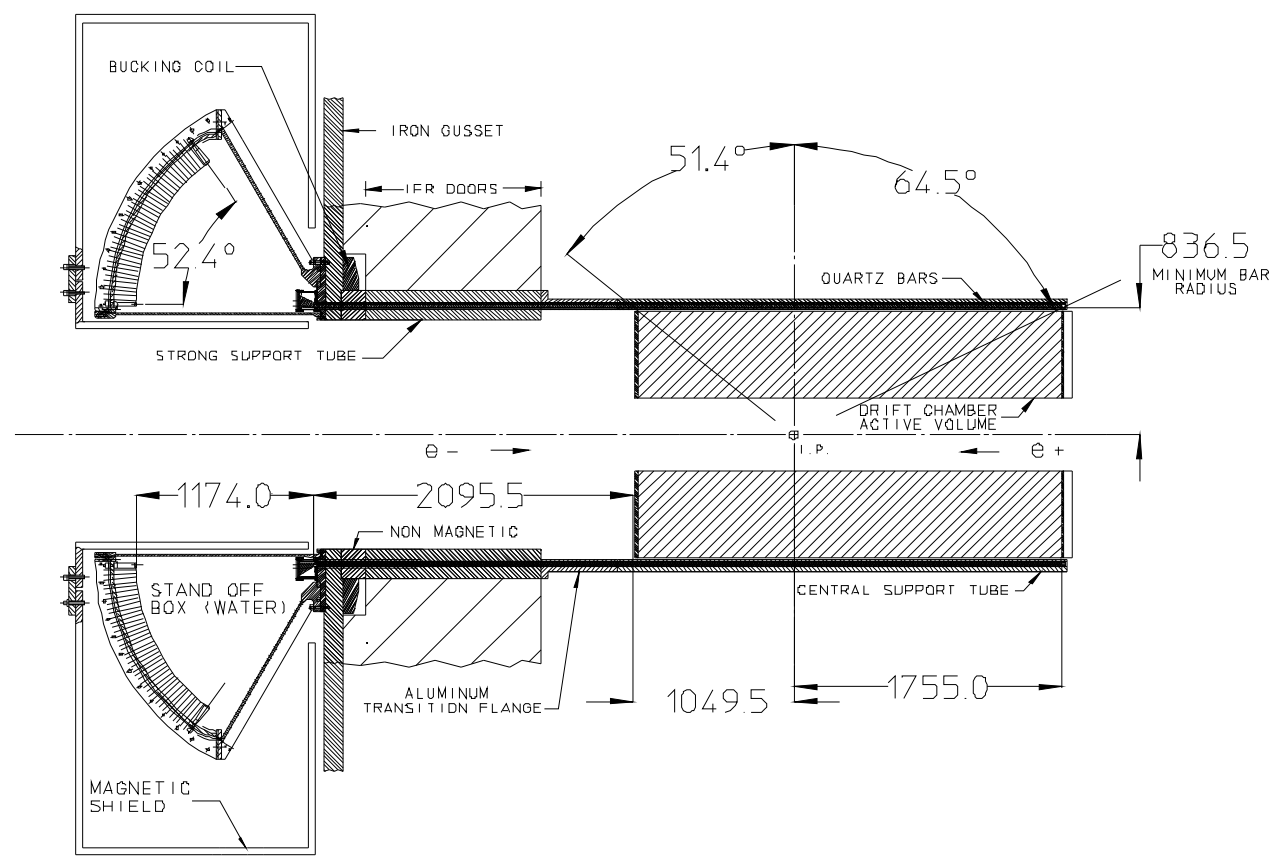

FIG. 2.13: Section transverse de l'architecture du DIRC.

de réduire le nombre de photo-multiplicateurs et de récupérer les photons qui seraient perdus par réflexion totale sur les parois. La surface du prisme la plus proche de l'axe longitudinal $z$ est inclinée de $6 \mathrm{mrad}$ afin d'améliorer la focalisation des photons sur les PM. En effet, cela réduit le déplacement relatif des photons subissant une réflexion sur cette partie du prisme, ce déplacement étant dû à l'épaisseur finie de la barre. Cet effet est schématisé sur la figure 2.12 : sont représentés deux photons de Cherenkov émis par une particule chargée. La trajectoire du photon qui ne subit pas de réflexion sur le prisme est en pointillés, alors que la trajectoire du photon qui subit une réflexion sur le prisme est en trait plein. Si la surface du bas du prisme n'était pas inclinée de $6 \mathrm{mrad}$, les deux photons auraient des directions de sortie parallèles et ne toucheraient donc pas le même photomultiplicateur. L'inclinaison du prisme permet de rabattre les photons ayant subit une réflexion en bas du prisme vers la direction qu'ils auraient eu sans cette réflexion.

Du côté instrumenté du DIRC, une cuve d'eau de $6 \mathrm{~m}^{3}$ appelée "standoff box" sert de volume d'expansion. On utilise de l'eau purifiée car elle a un indice proche de celui du quartz et cela minimise donc les pertes dues aux réflexions à la surface quartz/eau. De plus, l'eau pure est transparente à la lumière. Cependant, ce choix impose que les matériaux au contact de l'eau soient résistants à l'eau pure. Les photons sont détectés au bout de ce volume d'expansion par une série de tubes photo-multiplicateurs (PM) [41], de $2,82 \mathrm{~cm}$ de diamètre, soumis à une haute tension, pouvant être comprise entre 0,9 et $1,3 \mathrm{kV}$. Les tubes PM sont entourés par un cône capteur de lumière [42] pour minimiser les pertes de photons. L'efficacité de collection de la lumière par les cônes est de $90 \%$. Ces photo-multiplicateurs sont situés sur une surface de type torö̈dale à une distance de 1,2 m des barres. Il y a en tout environ 11000 photo-multiplicateurs. La standoff box est protégée par un blindage en fer doux et par une bobine dont le champ magnétique compense le champ de fuite du solénoïde dans la région des tubes photo-multiplicateurs. 
Le dispositif électronique $[43,44]$ enregistre la position du PM touché et le temps d'arrivée du photon sur les PM. Connaissant la direction de sortie des photons et l'angle d'incidence de la particule chargée dans la barre, on peut en déduire l'angle de Cherenkov $\theta_{C}$. La mesure temporelle permet de rejeter le bruit de fond machine entrant directement dans la standoff box. La résolution temporelle est de 1,7 ns.

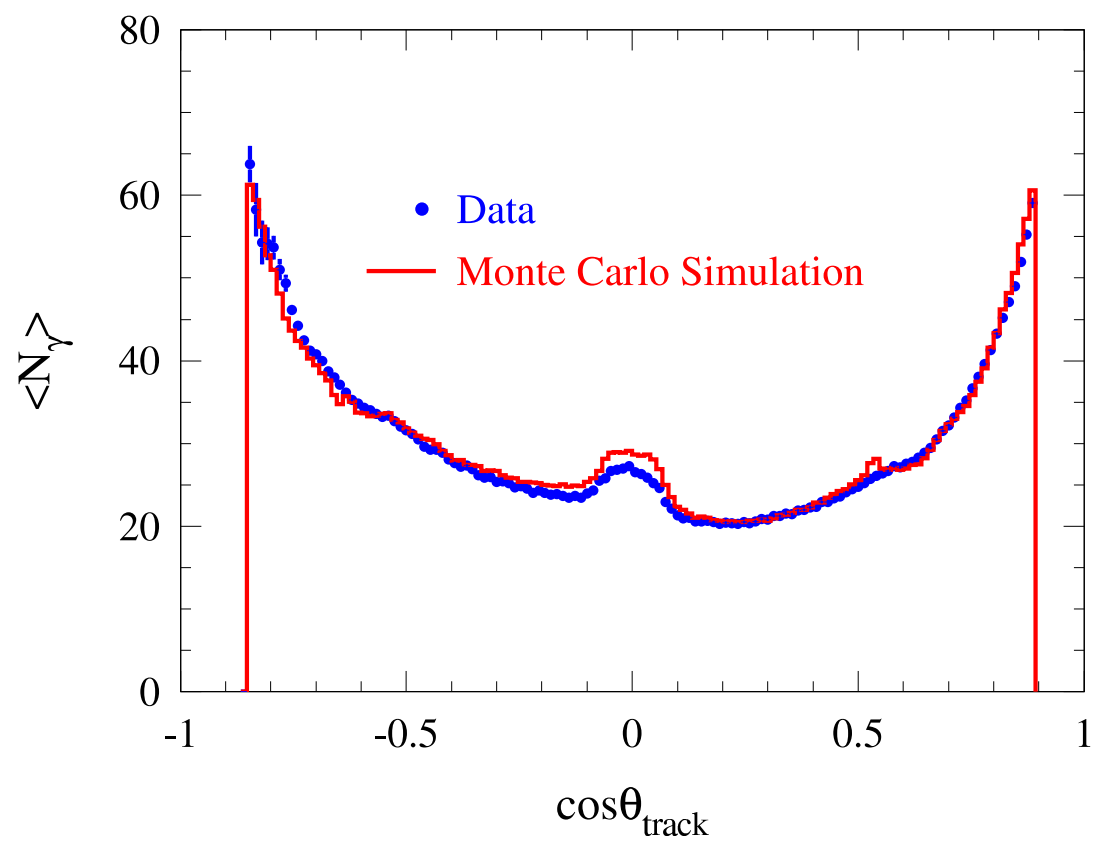

FIG. 2.14: Nombre de photons Cherenkov détectés en fonction de l'angle polaire des traces pour des événements $e^{+} e^{-} \rightarrow \mu^{+} \mu^{-}$. L'efficacité est meilleure vers l'avant et l'arrière qu'au centre car les photons sont alors émis avec des angles plus petits.

Une trace émet entre 20 et 60 photons dans le DIRC (voir Fig. 2.14). Le nombre de photons détectés dépend de la vitesse et de l'angle d'incidence de la particule. L'efficacité de détection d'un photon de Cherenkov par le DIRC est d'environ $5 \%$. Les photons de bruit de fond sont discriminés par leur temps d'arrivée aléatoire sur les photo-multiplicateurs. Si suffisamment de photons sont détectés, l'angle Cherenkov de la trace est ajusté par une méthode de maximum de vraisemblance à partir des $\theta_{C}$ des photons. Le programme de reconstruction donne une valeur de maximum de vraisemblance pour chaque type de particule "stable" (e, $\mu, \pi, \mathrm{K}, \mathrm{p})$, permettant ainsi l'identification de la particule. L'algorithme utilisé fournit pour chaque mesure l'angle $\theta_{C}$, le nombre de photons de signal et le nombre de photons de bruit de fond. En effet, ces deux dernières informations contribuent à l'identification d'une particule dont on connaît l'impulsion et l'angle de Cherenkov. Le nombre de photons attendu pour une particule d'impulsion et d'angle donnés a été déterminé sur Monte-Carlo. La fonction de vraisemblance utilisée pour l'identification des particules par le DIRC comporte deux parties utilisant toutes ces mesures. La première partie est gaussienne et compare l'angle de Cherenkov mesuré à la valeur théorique attendue pour chaque hypothèse de particule, tout en tenant compte de l'erreur sur la mesure de $\theta_{C}$. La gaussienne utilisée a pour expression : 


$$
g_{i}=\frac{1}{\sqrt{2 \pi \sigma_{\theta_{C}}^{2}}} \exp \left(-\frac{\left(\theta_{C}-\theta_{i}\right)^{2}}{2 \sigma_{\theta_{C}}^{2}}\right) .
$$

La deuxième partie utilise les nombres de photons de signal $N_{S}$ et de bruit de fond $N_{f}$ et suit une loi de Poisson :

$$
p_{i}=C_{i} \frac{\left(N_{i}+N_{f}\right)^{N_{S}+N_{f}} e^{-N_{i}-N_{f}}}{\left(N_{S}+N_{f}\right) !} .
$$

L'indice $i$ des équations 2.4 et 2.5 désigne l'hypothèse sur la particule (e, $\mu, \pi, \mathrm{K}, \mathrm{p}$ ), $\theta_{i}$ représente la valeur théorique de l'angle de Cherenkov pour chacune de ces hypothèses et $N_{i}$ le nombre de photons attendus. $C_{i}$ est un coefficient de normalisation. Ces fonctions de vraisemblances sont normalisées de la façon suivante:

$$
\sum_{i=1}^{5} g_{i}=1, \quad \sum_{i=1}^{5} p_{i}=1 .
$$

La fonction de vraisemblance associée au DIRC est donc :

$$
\mathcal{L}_{i}=\frac{g_{i} p_{i}}{\sum_{j=1}^{5} g_{j} p_{j}}
$$

Pour l'identification des particules, on combine les informations du SVT et de la chambre à fils. L'algorithme utilise donc des fonctions de vraisemblance gaussiennes qui utilisent la perte d'énergie par ionisation $d E / d x$ des particules chargées dans ces deux sous-détecteurs.

Le DIRC est un détecteur très performant. Il possède en outre l'avantage d'être très fin radialement ( $8 \mathrm{~cm}$ d'épaisseur) et a donc un impact minimal sur la détection des particules par le calorimètre électromagnétique (section 2.2.4). Son acceptance est de $87 \%$ d'angle solide polaire dans le référentiel du centre de masse, et sa couverture angulaire azimutale est de $93 \%$. Le seuil de détection des K est à $460 \mathrm{MeV} / \mathrm{c}$. La résolution de $\theta_{C}$ des photons est de l'ordre de 9,5 mrad, ce qui correspond à une résolution angulaire pour l'angle Cherenkov de la trace de l'ordre de $2,5 \mathrm{mrad}$, sachant que la différence de $\theta_{C}$ entre les $\pi$ et les $\mathrm{K}$ est de $6,5 \mathrm{mrad}$ pour une impulsion de $4 \mathrm{GeV} / c$. Le DIRC sépare les $\pi$ des $\mathrm{K}$ à $4,2 \sigma$ à $3 \mathrm{GeV} / \mathrm{c}$. La figure 2.15 illustre la très bonne séparation $K / \pi$ avec l'échantillon de contrôle $D^{*-} \rightarrow D^{0} \pi^{-}, D^{0} \rightarrow K^{-} \pi^{+}$. Sont également représentées sur ces figures les distributions attendues pour les différentes hypothèses utilisées dans l'algorithme d'identification.

La figure 2.16 montre la qualité de la séparation entre les $K$ et les pions en fonction de l'impulsion.

\subsubsection{Le calorimètre électromagnétique}

Le calorimètre électromagnétique [45] détecte les gerbes électromagnétiques produites par les particules qui le traversent et mesure leur position et leur énergie. Le calorimètre est utilisé aussi bien pour la reconstruction de photons de très basse énergie provenant 


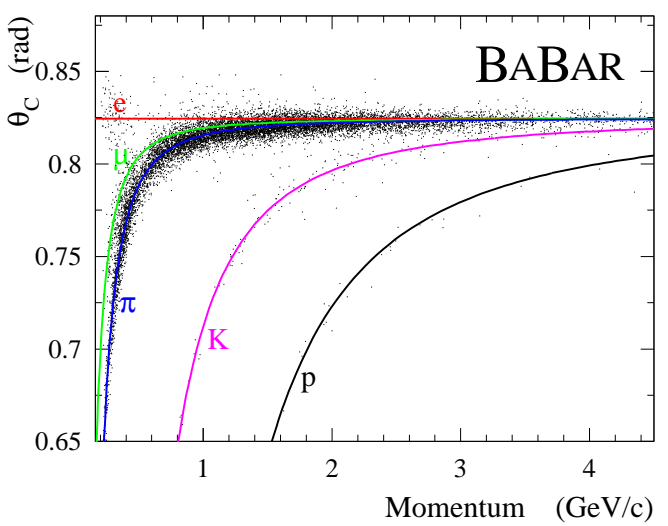

(a) Pions

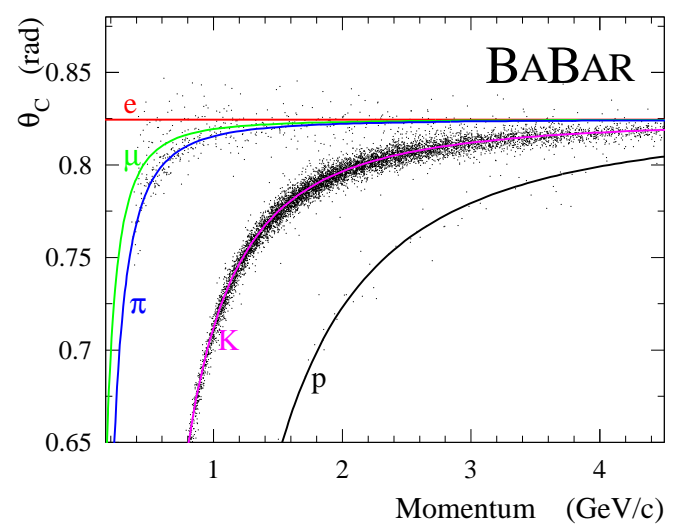

(b) Kaons

FIG. 2.15: Distribution de l'angle Cherenkov en fonction de l'impulsion pour les pions et les kaons chargés de l'échantillon de contrôle $D^{*-} \rightarrow D^{0} \pi^{-}, D^{0} \rightarrow K^{-} \pi^{+}$.

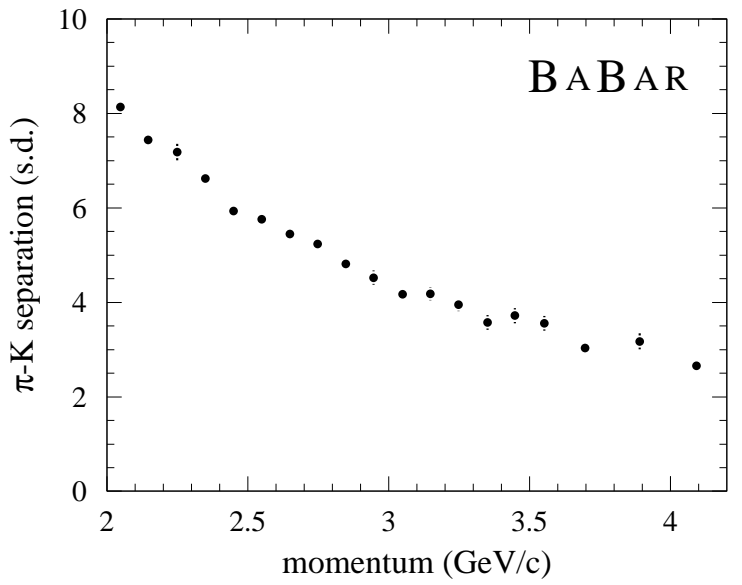

Fig. 2.16: Séparation des $\pi$ et des $K$ en nombre de déviations standards, en fonction de l'impulsion. 
de la désintégration d'un $\pi^{0}$ ou d'un $\eta$ que pour la reconstruction de photons de haute énergie produits par des processus électromagnétiques radiatifs. Il permet de détecter des particules d'énergie comprise entre $20 \mathrm{MeV}$ et $9 \mathrm{GeV}$ et couvre ainsi la quasi totalité du domaine d'énergie utile dans BABAR. Cependant il est inefficace pour détecter des particules de très faible énergie (inférieure à $20 \mathrm{MeV}$ ), car la mesure est alors dominée par le bruit de fond machine. Le calorimètre sert également à l'identification des électrons par la mesure du rapport E/p : ici, E désigne l'énergie déposée par la particule dans le calorimètre et $\mathrm{p}$ est l'impulsion de la particule, donnée par les informations combinées du détecteur de vertex et de la chambre à dérive. Cela permet en particulier de discriminer les électrons des pions. Le calorimètre est en particulier utilisé pour l'étiquetage de la saveur du $B_{\text {tag }}$ par un lepton.

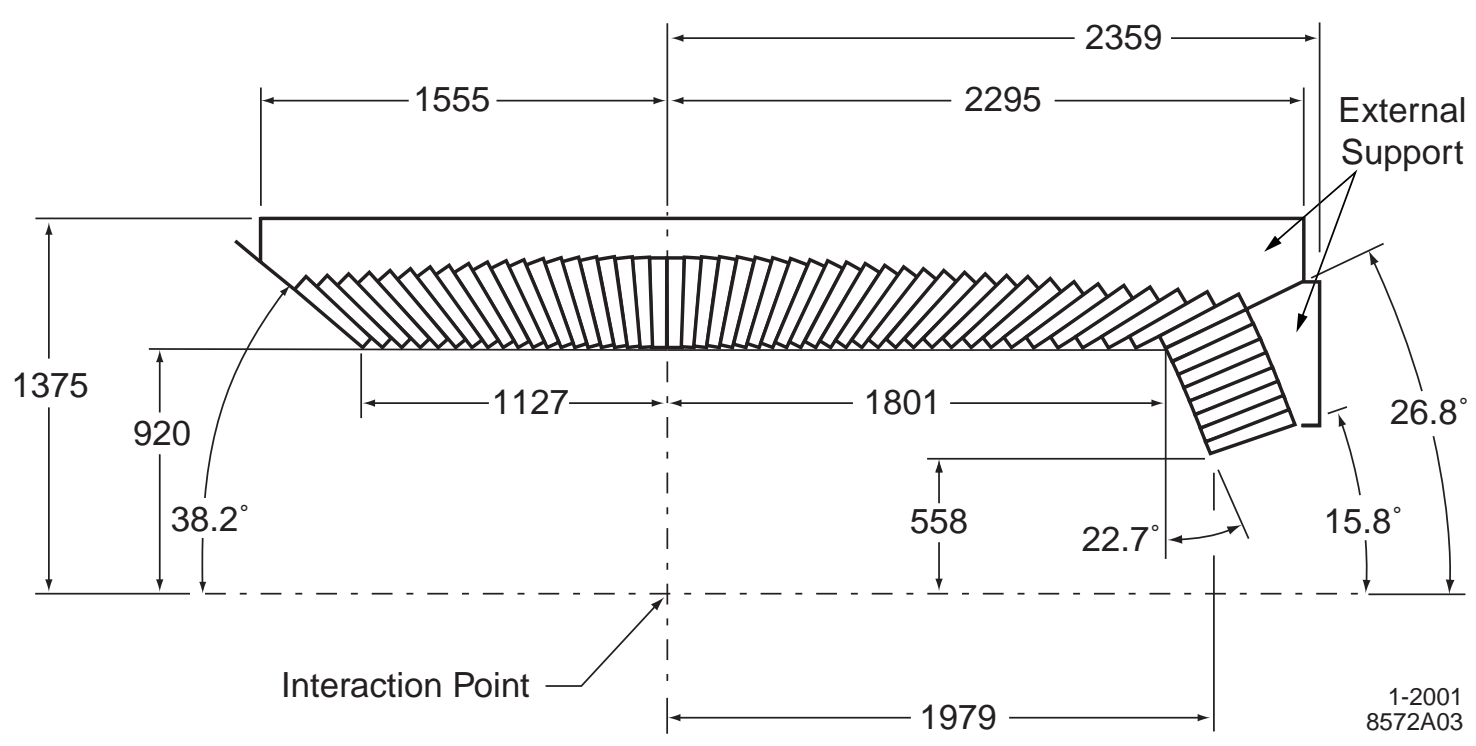

FIG. 2.17: Architecture schématique du calorimètre électromagnétique (les dimensions sont en mm.)

Le calorimètre consiste en un tonneau de forme cylindrique avec un bouchon conique vers l'avant, dans la direction où les particules sont préférentiellement émises, en raison de l'asymétrie du collisionneur (Fig. 2.17). Ses rayons internes et externes sont respectivement de $91 \mathrm{~cm}$ et $136 \mathrm{~cm}$. Le tonneau possède 5760 cristaux de scintillation, rangés en 48 colonnes selon l'angle polaire. Chaque colonne possède 120 cristaux selon l'angle azimutal. Le bouchon comprend 820 cristaux. Les cristaux sont regroupés en 280 modules faits de fibres de carbone composite de $300 \mu \mathrm{m}$ d'épaisseur. La lumière de scintillation produite par la particule électromagnétique qui traverse le calorimètre est lue à l'arrière par des photodiodes en silicium. Les cristaux utilisés sont du CsI(Tl), matériau qui permet une bonne production de lumière par dépôt d'énergie $(50000 \gamma / \mathrm{MeV})$ et dans laquelle le rayon de Molière est faible $(\sim 3,8 \mathrm{~cm})$ et la longueur d'interaction courte $(\sim 1,85 \mathrm{~cm})$. De forme trapézoïdale, les cristaux sont entourés de réflecteurs qui maximisent la lumière qui est collectée par deux photodiodes placées à l'arrière de chaque cristal. Ces propriétés permettent une détection efficace (proche de $100 \%$ à basse énergie) avec des dimensions relativement compactes. 
Le calorimètre est calibré régulièrement en utilisant des photons de $6.13 \mathrm{MeV}$ émis par des tubes contenant du fluocarbone excité par irradiation de neutrons. Ces tubes sont placés sur la face avant du calorimètre. Les Bhabhas sont également utilisés pour la calibration.

Une gerbe électromagnétique touche plusieurs cristaux adjacents dans le calorimètre, formant ainsi des amas. Un algorithme de reconnaissance de forme détermine ces amas à partir d'un cristal principal ayant une énergie de plus de $10 \mathrm{MeV}$. Il existe des amas avec un seul maximum d'énergie et des amas avec plusieurs maxima d'énergie. Dans ce cas-là, la position de la gerbe est déterminée en calculant le centre de gravité des différents maxima. Il convient également de distinguer les gerbes hadroniques des gerbes électromagnétiques. Pour cela, on utilise des variables de forme de gerbe dans l'algorithme, utilisant par exemple le fait qu'une gerbe d'un hadron touche plus de cristaux qu'une gerbe d'un photon, ou le fait qu'une gerbe électromagnétique est plus régulière. Les résolutions énergétique et angulaire du calorimètre, mesurées dans les données sur divers échantillons de contrôle, sont :

$$
\begin{aligned}
& \frac{\sigma_{E}}{E}=\frac{(2,32 \pm 0,30) \%}{\sqrt[4]{E(\mathrm{GeV})} \oplus(1,85 \pm 0,12) \%} \\
& \sigma_{\theta}=\sigma_{\phi}=\left(\frac{3,87 \pm 0,07}{\sqrt{E(\mathrm{GeV})}} \pm 0,04\right) \mathrm{mrad}
\end{aligned}
$$

Ces résolutions sont en relativement bon accord avec les résolutions déterminées par une simulation Monte Carlo (Fig. 2.18).

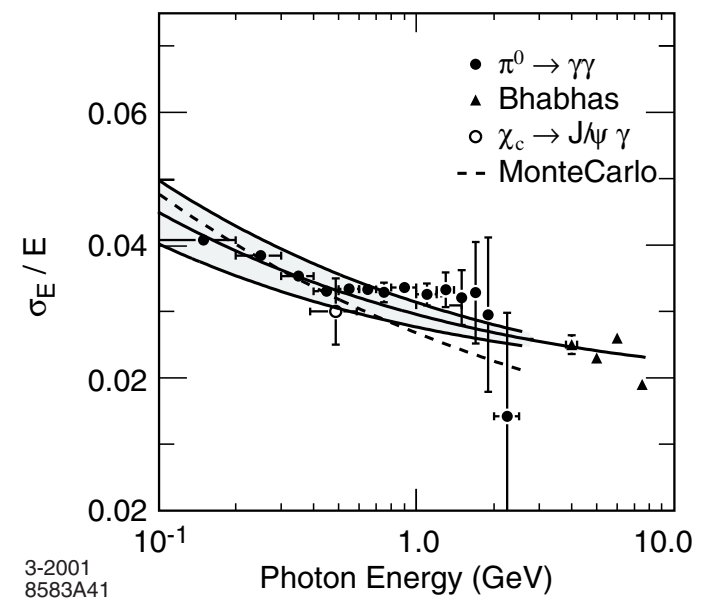

(a) Énergie

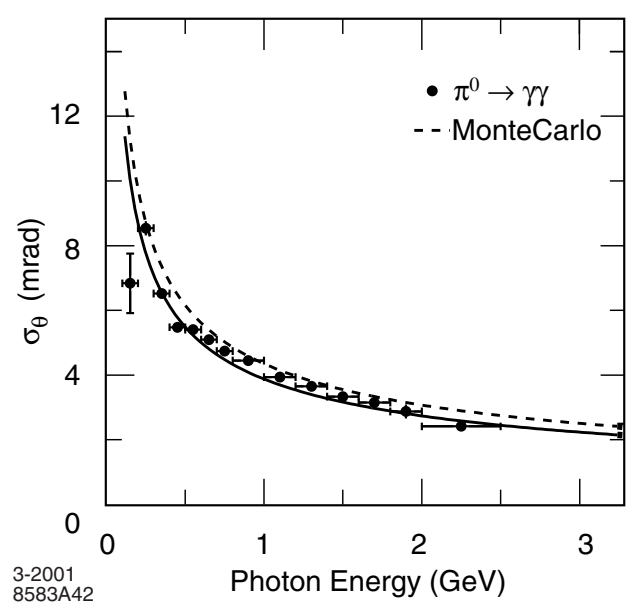

(b) Angle polaire

FIG. 2.18: Résolutions énergétique et angulaire du calorimètre en fonction de l'énergie déposée, mesurées dans les données par divers échantillons de contrôle, et comparées aux résultats d'une simulation Monte Carlo détaillée.

L'efficacité d'identification des électrons est de 94,8\% et la proportion de mauvaise identification des $\pi$ en électrons est de $0,3 \%$ (Fig. 2.19). La résolution en masse invariante des $\pi^{0}$ est de $6,9 \mathrm{MeV} / \mathrm{c}^{2}$ (Fig. 2.20). 

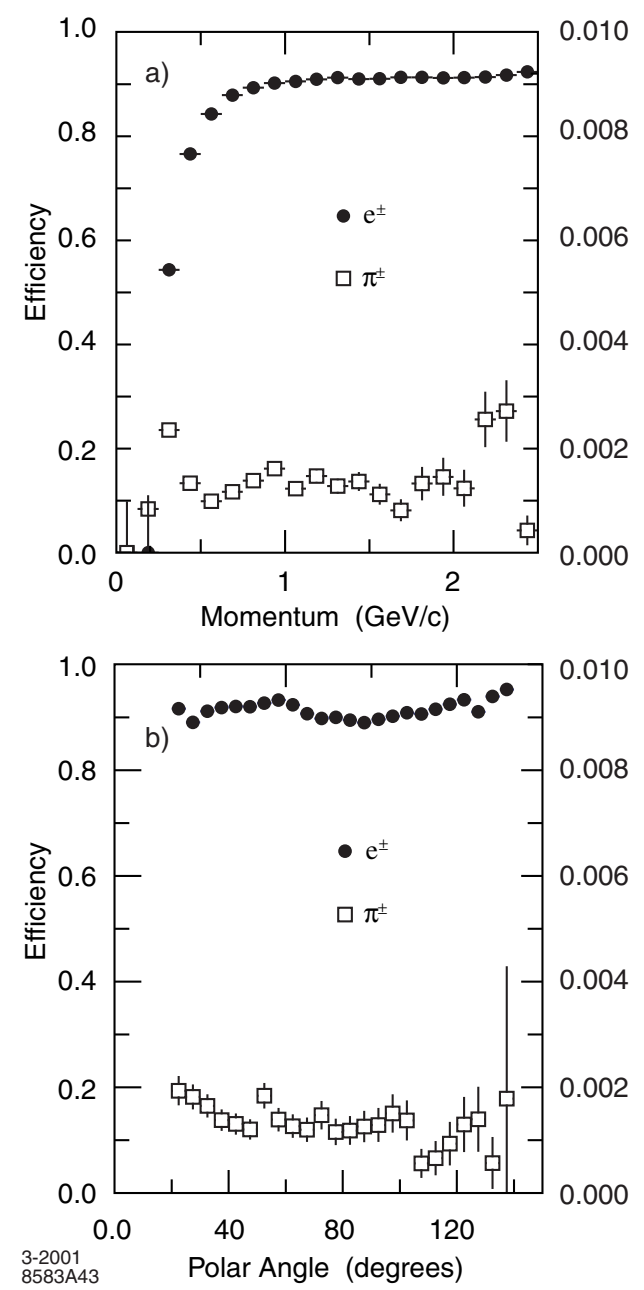

FIG. 2.19: Efficacité de détection des électrons (échelle de gauche) et efficacité des pions chargés (échelle de droite), en fonction de l'impulsion et de l'angle polaire des traces, dans le référentiel du laboratoire pour le calorimètre électromagnétique. 


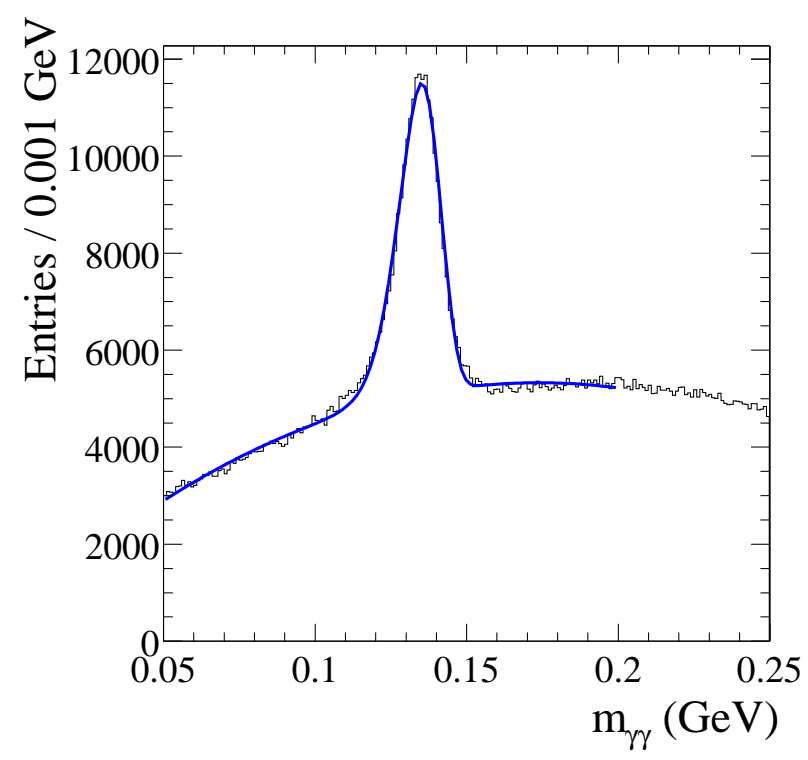

Fig. 2.20: Masse invariante des paires de photons provenant d'événements $B \bar{B}$. Les énergies des photons et celle du candidat $\pi^{0}$ reconstruit sont coupées hors de l'intervalle $[30,300 \mathrm{MeV}]$.

\subsubsection{Le solenoïde}

Le solenoïde [46] est un aimant supraconducteur placé après le calorimètre qui délivre un champ magnétique de 1,5 T. Il consiste en un cable de Rutherford de $10 \mathrm{~km}$, composé de filaments de NbTi incrustés dans de l'aluminium. Le courant dans le solenoïde est de 4600 A. De l'hélium liquide le maintient à $4.5 \mathrm{~K}$. Son rôle est de courber la trajectoire des particules chargées, en particulier dans le détecteur de vertex et dans la chambre à dérive, permettant ainsi la mesure de leur impulsion.

L'aimant a été construit de façon à répondre à certaines contraintes. Il doit être peu encombrant de manière à ne pas détériorer la détection des hadrons neutres et des muons par l'IFR (voir partie 2.2.6). La contrainte principale consiste à ne pas détériorer les performances de PEP-II. En particulier, les aimants Q2, Q4 et Q5 ne doivent pas être exposés à un champ magnétique supérieur à $0,01 \mathrm{~T}$. Un retour de champ segmenté en acier a été placé sur le solénoïde afin de protéger ces aimants. Il est constitué d'un tonneau de forme hexagonale et de deux bouchons. Ce retour de champ est également utilisé pour accueillir l'IFR (section 2.2.6).

Il est également nécessaire que le champ magnétique soit uniforme, ce qui est vrai à mieux que $2 \%$, comme l'illustre la figure 2.21 .

\subsubsection{Le retour de champ instrumenté : IFR (Instrumented Flux Return)}

L'IFR [47] est principalement un détecteur à $\mu$, particulièrement pur et efficace pour des impulsions supérieures au $\mathrm{GeV} /$ c. Les $\mu$ sont utilisés pour l'étiquetage de saveur des B mais aussi pour l'étude des désintégrations semi-leptoniques du B. L'IFR permet éga- 


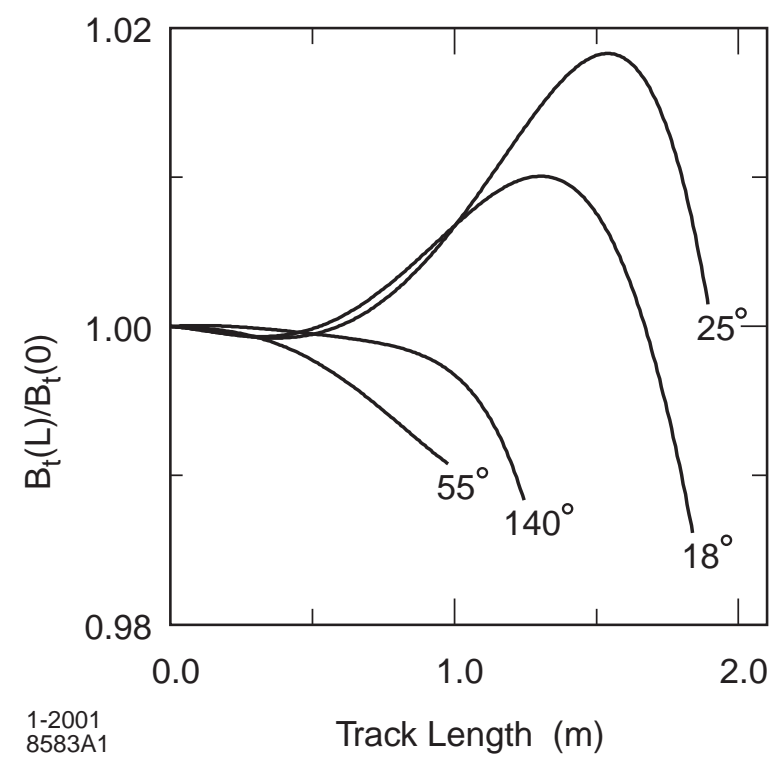

Fig. 2.21: Amplitude relative du champ magnétique transverse le long de la trajectoire d'une particule de haute impulsion en fonction de sa distance du point d'interaction et de son angle polaire.

lement la détection des hadrons neutres $\left(K_{L}^{0}, n\right)$ et mesure leur impulsion. Enfin, il peut aider au veto des désintégrations charmées et améliorer la reconstruction des $\nu$. L'information de l'IFR est combinée aux informations fournies par les autres sous-détecteurs. La détection des $\mu$ par l'IFR nécessite une bonne couverture d'angle solide, une bonne efficacité et une bonne rejection du bruit de fond. En ce qui concerne les hadrons neutres, une bonne efficacité et une bonne couverture angulaire sont primordiales.

L'IFR utilise le retour de flux en acier segmenté du solenoïde comme filtre à $\mu$ et absorbeur d'hadrons. Le détecteur consiste en une série de RPC (Resistive Plate Chamber) $[48,49]$ situées dans les interstices de l'acier qui constitue le tonneau et les bouchons du retour de champ. Il y a 19 couches de RPC planes dans le tonneau et 18 dans les bouchons. Deux couches supplémentaires sont situées entre le calorimètre et l'aimant. Les RPC sont faites de deux couches de bakelite de $2 \mathrm{~mm}$ d'épaisseur séparées par une fente de $2 \mathrm{~mm}$, remplie d'un gaz ininflammable, composé de 56,4\% d'argon, de 38,8\% de freon et de 4,5 $\%$ d'isobutane (Fig. 2.22). De part et d'autre de cet ensemble se trouve une surface de graphite. L'une d'elle est sous haute tension $(8 \mathrm{kV})$ et l'autre est à la terre, afin de créer un champ électrique uniforme. Ce détecteur couvre en tout une surface de $2000 \mathrm{~m}^{3}$. Il y a 806 RPC regroupées en modules dont la taille dépend de l'espace dans lequel ils sont placés.

Les RPC détectent le passage d'une particule ionisante dans le gaz, développant ainsi une étincelle entre les deux plaques. Cela induit un signal capacitif lu par des pistes en aluminium disposées en un maillage bidimentionnel, selon les coordonnées $(\mathrm{z}, \phi)$ dans le tonneau et $(\mathrm{x}, \mathrm{y})$ dans les bouchons. Un système de lecture électronique situé en dehors des RPC lit la tension déposée par l'impulsion électrique sur les pistes d'aluminium. Connaissant les pistes touchées, on peut en déduire la position de l'impact. La résistivité 


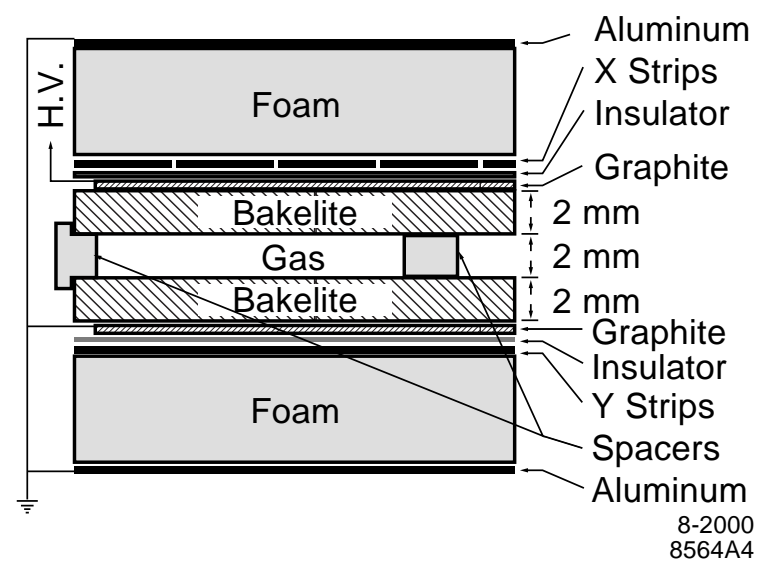

FIG. 2.22: Représentation schématique d'une RPC.

des plaques est telle que le champ électrique est instantanément éteint au voisinage de l'étincelle. Ainsi, celle-ci ne se propage pas au reste de la chambre. De plus le gaz a des propriétés absorbantes dans le domaine de l'UV, ce qui empêche les photons de se propager. Ce dispositif assure donc une très bonne résolution sur le temps du point d'impact. L'efficacité de détection des muons d'impulsion comprise entre 1,5 et $3 \mathrm{GeV}$ est de $90 \%$ et la probabilité de mauvaise identification des pions est de l'ordre de 6 à $8 \%$ (voir Fig. $2.23)$.

Depuis le démarrage, l'efficacité des RPC s'est dégradée. Cependant, leur très grand nombre fait que les conséquences de cette perte d'efficacité n'est pas dramatique. Les RPC des bouchons ont été remplacées en 2002. Il est prévu de remplacer les RPC du tonneau par des LST (limited streamer tubes). Cela a été fait pour deux des six secteurs du tonneau en 2004.

\subsubsection{Le système de déclenchement}

La haute luminosité de PEP-II fait qu'il y a de nombreuses particules dans le détecteur à chaque instant. Le rôle du système de déclenchement est de sélectionner les événements de physique avec une haute efficacité tout en rejetant un maximum de bruit de fond. Ainsi, seuls les événements susceptibles d'avoir un intérêt physique sont enregistrés. Le système de déclenchement de BABAR se fait en deux étapes. Le premier niveau de déclenchement (L1) est un système électronique qui agit en temps réel au moment de la prise de données. Si certains critères de sélection sont remplis, l'événement est déclenché et enregistré temporairement. Le deuxième niveau de déclenchement (L3) est un programme informatique qui fait une reconstruction rapide des événements enregistrés au niveau L1. Des critères de sélections permettent alors de déterminer si l'événement est enregistré sur disque ou pas.

Le principe du système de déclenchement L1 est de déterminer si une particule est détectée. Pour cela, les informations de la chambre à dérive, du calorimètre et éventuellement de l'IFR sont utilisées. Le système de déclenchement est basé sur les courbes d'acceptance des objets individuels, qui sont les traces chargées dans la chambre à dérive 

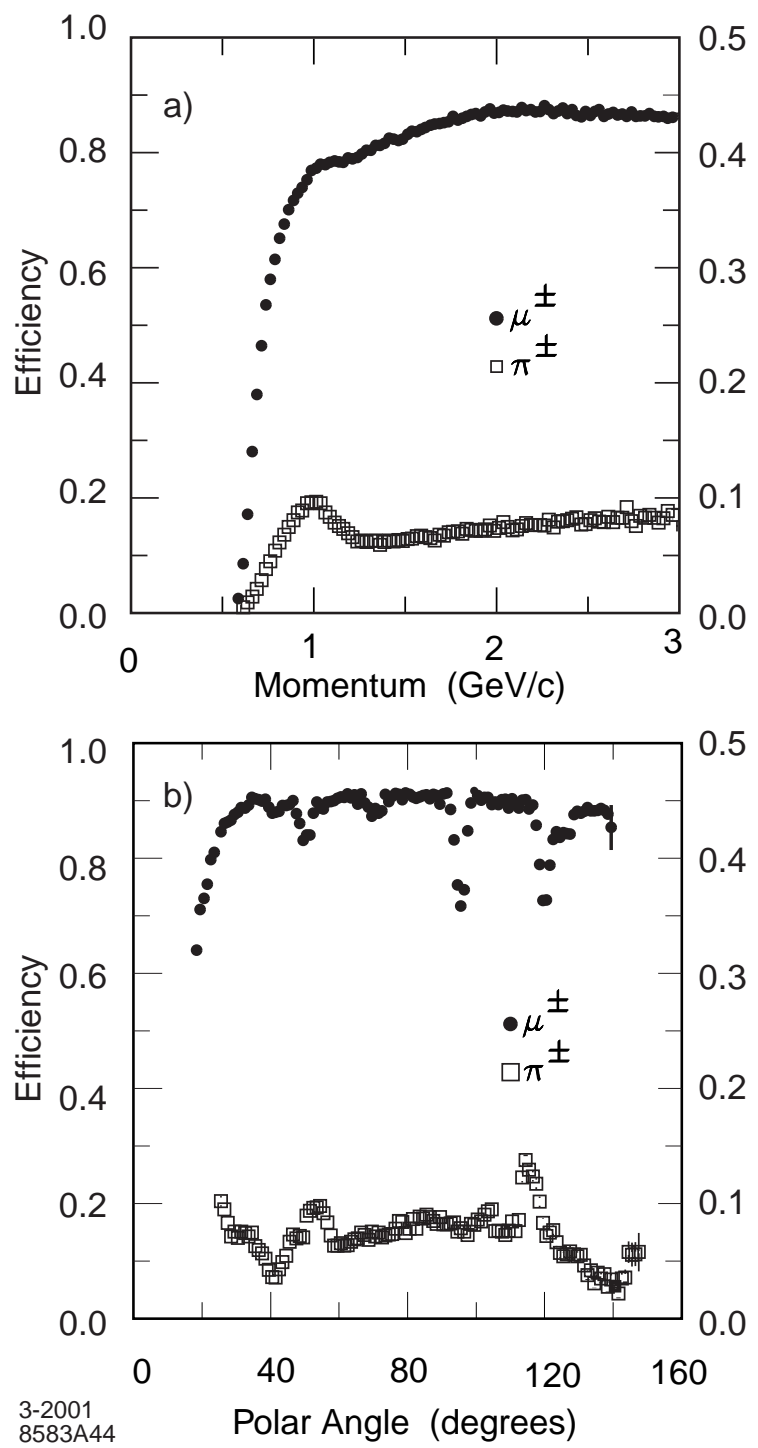

FIG. 2.23: Efficacité de détection des muons (échelle de gauche) et efficacité des pions chargés (échelle de droite), en fonction de l'impulsion et de l'angle polaire des trace, dans le référentiel du laboratoire avec l'IFR. 
ou les dépôts d'énergie dans le calorimètre. Le système de déclenchement global utilise une combinaison de ces objets avec des critères sur leur nombre et éventuellement sur leur séparation azimutale et sur la correspondance entre les traces et les dépôts d'énergie. La fréquence de déclenchement du niveau L1 ne dépasse pas $2 \mathrm{kHz}$. Le bruit de fond peut être réduit en imposant des coupures supplémentaires sur l'impulsion transverse des traces ou sur l'énergie déposée dans le calorimètre. Le niveau L3 permet une meilleur rejection du bruit de fond machine et des événements Bhabhas, en utilisant par exemple des critères sur la coordonnée longitudinale du point de plus proche approche des traces chargées reconstruites. La fréquence de déclenchement de L3 est inférieure à $120 \mathrm{~Hz}$. Le système de déclenchement total a une efficacité de $99,7 \%$ pour les événements $B \bar{B}$. 
Chapitre 2. Le dispositif expérimental : BABAR et PEP-II 


\section{3 \\ L'alignement longitudinal du DIRC}

\section{Introduction}

Ainsi que cela a été expliqué au chapitre précédent, le DIRC a été conçu afin d'identifier les particules, en en particulier afin de séparer les kaons des pions. Pour cela, le DIRC utilise le rayonnement Cherenkov émis par les particules qui traversent les barres de quartz. En pratique, cela revient à mesurer l'angle de Cherenkov $\theta_{C_{i}}$ des photons $i$ émis par une particule qui traverse le DIRC. La résolution géométrique de reconstruction de $\theta_{C}$ peut être estimée à partir des dimensions des différentes parties du DIRC (distance parcourue dans la standoff box, dimensions des tubes photo-multiplicateurs et des barres). Cette résolution est de l'ordre de $7 \mathrm{mrad}$. Elle est détériorée par la dispersion des photons dans la barre $(\sim 3 \mathrm{mrad})$ et par l'achromaticité de production des photons de Cherenkov $(\sim 5,5 \mathrm{mrad})$. La résolution attendue est donc de l'ordre de $10 \mathrm{mrad}$ (Fig 3.1).

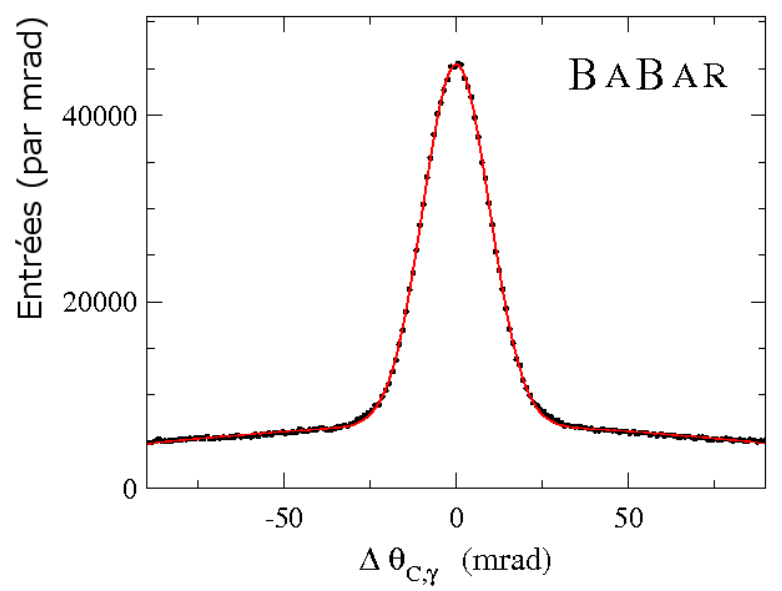

FIG. 3.1: Résolution sur l'angle de Cherenkov des photons de Cherenkov dans le DIRC pour des traces chargées provenant d'événements multihadroniques. La distribution est ajustée avec la somme de deux Gaussiennes : la plus étroite est large de $9.6 \mathrm{mrad}$ et représente $90 \%$ de la hauteur du pic.

Pour chaque photon $i$ détecté, on reconstruit un angle de Cherenkov $\theta_{C_{i}}$. On peut alors 
ajuster l'angle Cherenkov $\theta_{C}$ de la particule chargée à partir des tous les $\theta_{C_{i}}$ des photons qu'elle a émis. La résolution sur l'angle Cherenkov de la trace est alors de l'ordre de 2,9 mrad lorsqu'il n'y a pas d'alignement du DIRC. Cette résolution peut être significativement améliorée par un meilleur alignement du DIRC.

\subsection{Caractérisation de l'alignement du DIRC}

La reconstruction du DIRC dépend de la géométrie même de ce détecteur et comporte plusieurs étapes :

- On connaît la position du tube photo-multiplicateur touché et la barre d'où est originaire le photon de Cherenkov et on peut donc déterminer la direction de sortie du photon. L'erreur sur cette direction est directement liée aux dimensions du prisme en sortie de la barre et du tube photo-multiplicateur.

- Connaissant de plus la différence d'indice entre l'eau purifiée de la standoff box et le quartz des barres, on en déduit la direction du photon dans la barre, en supposant un alignement parfait et en utilisant le fait que l'angle de réflexion du photon sur les parois de la barre est conservé.

- On connaît d'autre part la direction de la trace à l'entrée du DIRC, grâce aux informations du détecteur de vertex et de la chambre à fils. On peut alors calculer l'angle d'émission $\theta_{C_{i}}$ du photon de Cherenkov par rapport à la trace chargée.

Il existe cependant certaines ambiguïtés sur la mesure de $\theta_{C_{i}}$ comme la parité du nombre de réflexions dans la barre, la direction par laquelle le photon est sorti du prisme (gauche ou droite), le fait que le photon ait été ou non réfléchi par le miroir en bout de barre, le fait que le photon ait été rabattu par le prisme ou non. Certaines ambiguïtés peuvent être levées en utilisant le temps d'arrivée du photon sur les PM, les contraintes géométriques du DIRC et des hypothèses de masse de la particule qui émet le rayonnement de Cherenkov. La principale source de détérioration de la résolution sur $\theta_{C}$ provient du mauvais alignement du DIRC. En effet, on veut mesurer $\theta_{C}$ avec une très bonne précision de l'ordre de quelques mrad et donc tout mauvais positionnement des éléments du DIRC peut contribuer à la dégradation de la résolution.

Il convient également que la position relative du DIRC soit bien déterminée par rapport au reste du détecteur, et que le détecteur de vertex soit lui aussi bien aligné. Il est important de pouvoir refaire l'alignement du DIRC régulièrement et facilement, afin de tenir compte à la fois des éventuelles déformations du DIRC lui-même, mais aussi du détecteur de vertex, l'alignement de ce dernier étant recalculé régulièrement. Des études ont d'ailleurs confirmé la dépendance de l'alignement de DIRC en fonction de l'alignement du SVT. Un meilleur alignement du SVT conduit à l'amélioration significative des performances du DIRC.

Le DIRC est constitué de nombreuses sous-parties : les barres, elles-même constituées de 4 segments collés bout-à-bout, le prisme, le miroir et la standoff box (figure 3.2). Les positions de ces différents éléments ont été déterminées au moment de la construction du DIRC par une sonde optique. Cependant, il est possible que cet alignement ne soit pas parfait. Il est tout d'abord limité par la précision de l'assemblage. De plus, des contraintes mécaniques ou le poids des barres peuvent induire des déformations barre à barre. Un 
alignement par un programme informatique agissant au moment de la reconstruction de l'angle Cherenkov des photons est donc nécessaire.

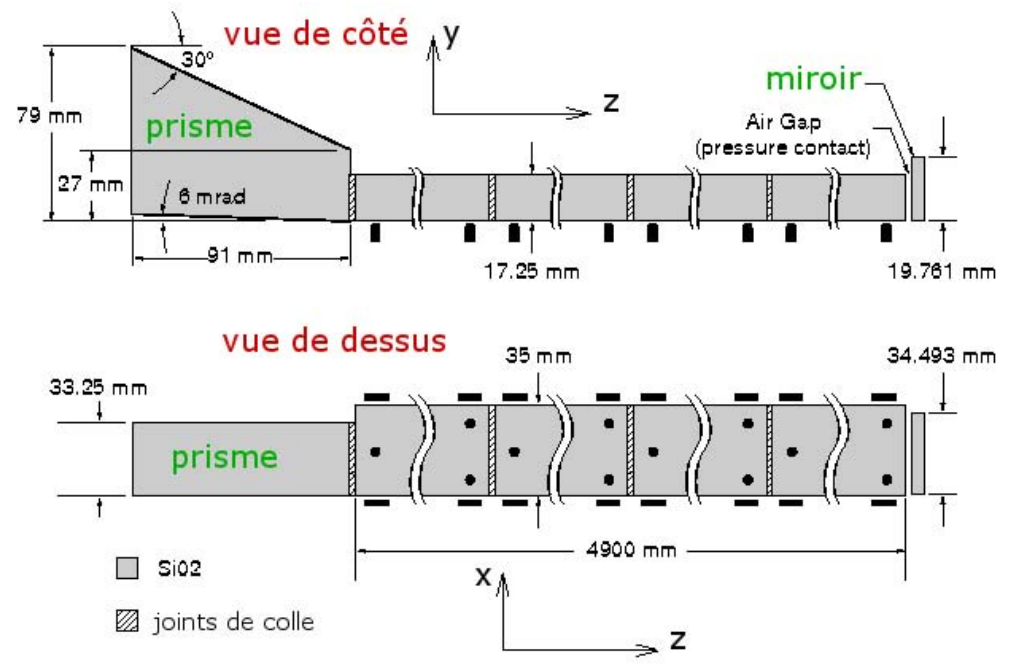

FiG. 3.2: Description schématique de barres de quartz du DIRC.

Le DIRC mesure à la fois l'angle Cherenkov $\theta_{C}$ et l'angle azimutal $\phi_{C}$ des photons émis par rayonnement Cherenkov dans les barres du DIRC. Un alignement dans le plan transverse permet d'améliorer la précision sur la mesure de $\phi_{C}$ et sur la définition du volume de détection. Cependant, cela n'affecte pas directement la mesure de $\theta_{C}$, qui est l'angle le plus important à déterminer. Un alignement transverse qui utilise les mesures des positions transverses des éléments du DIRC par la sonde optique a permis d'améliorer la résolution sur $\theta_{C}$ de $0,04 \mathrm{mrad}$. On ne s'attend donc pas à un gain significatif sur la résolution grâce à un nouvel alignement transverse. En revanche, un alignement longitudinal est beaucoup plus important, car cela affecte directement la mesure de $\theta_{C}$. Il est en particulier primordial de bien connaître l'angle d'inclinaison des constituants de la barre (segments et prismes). En effet, on note une forte dépendance de la mesure de $\theta_{C}$ en fonction de la position longitudinale d'entrée des traces dans la barre $(z)$ ou de l'angle d'inclinaison de la trace dans la barre. De plus, cette dépendance varie selon les barres. Cela conduit à penser que le mauvais alignement du DIRC n'est par dû à un mauvais positionnement global des barres. Il est donc nécessaire d'aligner le DIRC barre par barre. Avec un tel alignement, on s'attend à une amélioration significative de la résolution de $\theta_{C}$, de l'ordre de $0,2 \mathrm{mrad}$.

\subsection{Stratégies envisagées pour l'alignement longitu- dinal du DIRC}

Pour chaque barre de quartz, les possibilités de mauvais alignement sont de deux types. Il peut s'agir soit d'un déplacement global de la barre (translation ou rotation), soit d'une déformation mécanique de la barre, comme par exemple une flèche due à la gravité. Dans 
le programme de reconstruction utilisé, les barres sont définies comme étant des solides indéformables. Il est donc facile de corriger les effets d'un mauvais alignement global. En revanche, estimer et simuler les déformations mécaniques ou ajuster les positions des différents composants de chaque barre est impossible dans le code utilisé. Il est par exemple possible que les différents segments de la barre ne soient pas parfaitement alignés au niveau des joints de colle.

L'observable utilisée pour estimer la qualité de l'alignement du DIRC est le résidu de l'angle de Cherenkov de chaque photon, défini comme la différence entre l'angle de Cherenkov mesuré et l'angle de Cherenkov attendu dans un échantillon de contrôle. Pour cela, on utilise des échantillons de di-muons, produits par le processus $e^{+} e^{-} \rightarrow \mu^{+} \mu^{-}$. Utiliser des événements di-muons est particulièrement bien adapté au type d'étude que l'on souhaite faire. En effet, on dispose d'un grand nombre d'événements di-muons, ces événement sont bien identifiés, leur distribution d'angle Cherenkov en fonction de l'impulsion est bien connue et leur signal dans le DIRC est propre, car il n'y a que deux traces dans l'événement. Les di-muons représentent donc un excellent échantillon pour aligner le DIRC. La distribution de résidus peut être ajustée par une gaussienne. La valeur moyenne de la gaussienne quantifie le biais systématique éventuel sur la mesure de l'angle de Cherenkov et la largeur de la gaussienne quantifie la résolution. Si l'alignement est bon, la distribution de résidus doit être centrée en 0 et la résolution doit être minimale.

Dans un premier temps, on a essayé d'appliquer des corrections globales sur les positions de chacune des barres, telles qu'une translation selon l'axe du faisceau $z$, ou selon l'axe $y$, orthogonal à $z$ dans le plan longitudinal de la barre ou telles qu'une rotation autour du troisième axe $x$. Or l'alignement doit être bon dans tout le domaine d'acceptance du DIRC, c'est-à-dire pour toutes les valeurs d'angle d'inclinaison de la trace dans une barre. L'angle d'inclinaison $\lambda$ est défini par :

$$
\lambda=\frac{\pi}{2}-\theta,
$$

où $\theta$ est l'angle polaire de la trace. C'est pourquoi il est intéressant de considérer la distribution de résidus par barre en fonction de $\lambda$. Des études utilisant ce type de distributions ont permis de montrer qu'appliquer des corrections globales aux barres n'était pas suffisant pour obtenir un bon alignement. Il était donc nécessaire d'appliquer des corrections plus fines. Une solution est de construire une table de correction, dont le principe est de corriger l'angle de Cherenkov de chaque photon, en fonction de sa direction dans la barre.

\subsection{Alignement par une table de correction}

\subsubsection{Principe}

Le principe de l'alignement par une table de correction consiste à calculer une correction sur la mesure de l'angle de Cherenkov $\theta_{C}$ de chaque photon en fonction de sa direction dans la barre. Pour cela on utilise des événements di-muons. L'intérêt de corriger l'angle Cherenkov des photons plutôt que de corriger directement l'angle de la trace en fonction de son angle d'inclinaison dans la barre est que l'on s'affranchit des biais dûs au choix de 
l'échantillon de contrôle utilisé pour calculer les corrections. Les photons émis par rayonnement Cherenkov par un muon le sont dans toutes les directions et constituent donc un échantillon complet pour l'alignement. La direction d'un photon est décrite par un vecteur orthonormé $\vec{k}$, de coordonnées $\left(k_{x}, k_{y}, k_{z}\right)$ dans le repère $(x, y, z)$ de la barre. L'axe $z$ est dans la direction longitudinale, et les axes $x$ et $y$ déterminent le plan transverse comme le montre la figure 3.3 .
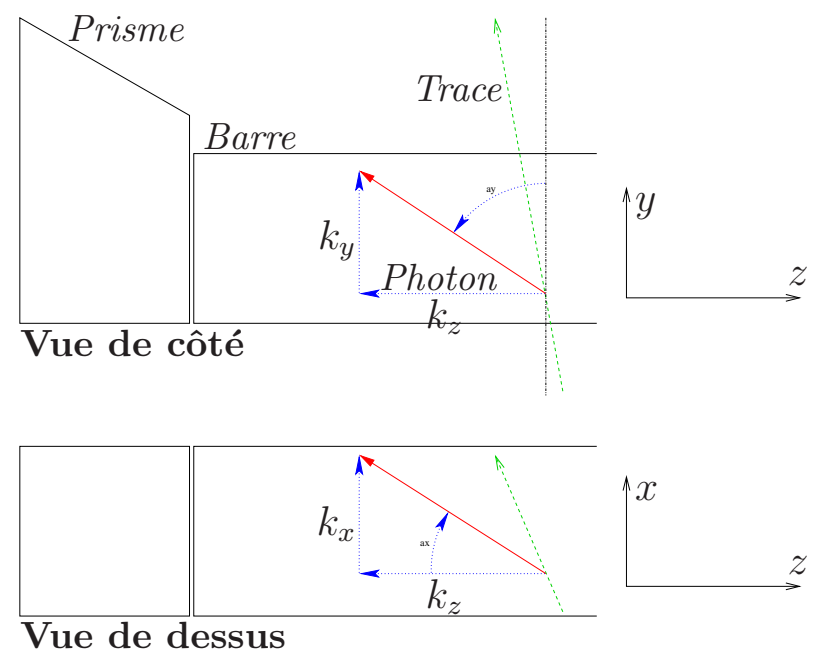

FIG. 3.3: Définition du repère $(x, y, z)$ dans une barre

La table combine toutes les informations nécessaires pour définir la direction d'un photon dans une barre, à savoir :

- le numéro de la barre traversée par la particule chargée qui émet les photons de Cherenkov

- les coordonnées $k_{x}$ et $k_{y}$ du vecteur direction du photon dans la barre : pour chacune de ces coordonnées, on calcule une correction dans 15 intervalles compris entre $-0,75$ et 0,75

- le signe de $k_{z}$ qui indique si le photon a été réfléchi ou pas par le miroir situé au bout de la barre

- la direction par laquelle le photon est sorti du prisme : gauche ou droite

Toutes ces informations sont nécessaires et suffisantes pour permettre de déterminer sans ambiguïté la direction d'un photon de Cherenkov. Il est impératif de calculer les corrections par barre, car il n'y a aucune raison pour que les barres soient déformées de la même façon. La combinaison des autres variables permet de lever toutes les ambiguités inhérentes au principe même du DIRC sur la direction du photon. La grande statistique de PEP-II a permis de calculer des corrections avec une granularité très fine sur la direction du photon. On a en effet en tout 129600 bins.

Cette méthode d'alignement a été utilisée en 2000 et avait alors permis d'améliorer significativement la résolution sur la mesure de l'angle Cherenkov des traces, qui a été diminuée de près de 0,3 mrad. Cette table avait été calculée à partir des données du RUN 1. Elle a été introduite dans la base de données en 2001 et a été utilisée pour la reconstruction des données jusqu'au RUN 3. Les données de 1999 et 2000 ont également été à nouveau reconstruites avec cette table. Si cet alignement était performant, il n'en 
était pas moins important de le refaire afin de vérifier qu'il ne s'était pas dégradé. Il est peu vraisemblable que les différents éléments du DIRC aient significativement bougé les uns par rapport aux autres. Cependant, la qualité de l'alignement du DIRC est corrélée à celle du SVT. En effet, le DIRC permet uniquement de déterminer la direction des photons de Cherenkov dans chaque barre. C'est en extrapolant les mesures du SVT afin de déterminer la direction de la particule chargée à l'entrée du DIRC que l'on en déduit l'angle Cherenkov des photons. Cette mesure est donc très dépendante de l'alignement du SVT. Cela a été vérifié lors du premier alignement. Une nouvelle table de correction a donc été construite avec les données du RUN 3, et le code informatique correspondant a été refait de façon à ce que l'alignement puisse être recalculé plus régulièrement.L'alignement a été calculé en utilisant un échantillon de di-muon d'une luminosité intégrée de $11 \mathrm{fb}^{-1}$, ce qui correspond à environ 13 millions de traces et 350 millions de photons.

\subsubsection{Procédure pour calculer les corrections}

Chaque entrée de la table de correction est calculée de la façon suivante :

- La distribution de résidus d'angle de Cherenkov des photons correspondant à cette entrée est stockée dans un histogramme.

- chaque distribution est alors ajustée par la somme d'une gaussienne et d'une droite. La droite permet de modéliser le bruit de fond. La correction correspond à la valeur du biais de la gaussienne. Si l'alignement était parfait, le biais serait nul. La figure 3.4 représente un exemple typique d'ajustement d'une distribution de résidus.

Les valeurs des corrections ainsi calculées sont comprises entre -10 mrad et $10 \mathrm{mrad}$. La figure 3.5 représente les corrections calculées pour quelques barres. Le vecteur $\vec{k}$ étant normé, certaines valeurs de $k_{x}$ et de $k_{y}$ ne peuvent pas être atteintes, ce qui explique pourquoi certains bins n'ont pas d'entrées.

La correction peut être améliorée si on corrige une nouvelle fois les photons corrigés. Dans cette deuxième étape, les résidus sont pondérés par un poids identique à celui intervenant dans l'ajustement de l'angle Cherenkov de la trace $\theta_{C}$ à partir des angles Cherenkov des photons $\theta_{C_{i}}$ :

$$
w=\exp \left(-\frac{\left(\theta_{C_{i}}-\theta_{C}\right)^{2}}{2 \sigma_{\theta_{C}}^{2}}\right)
$$

où $\sigma_{\theta_{C}}$ est l'erreur sur $\theta_{C}$, fournie par l'algorithme de reconstruction. Dans ce cas-là, la distribution est ajustée uniquement par une gaussienne. Cette deuxième correction est très faible par rapport à la première et est surtout utile pour améliorer l'alignement de quelques barres en particulier. La correction globale appliquée aux photons de Cherenkov est donc la somme de ces deux corrections. 


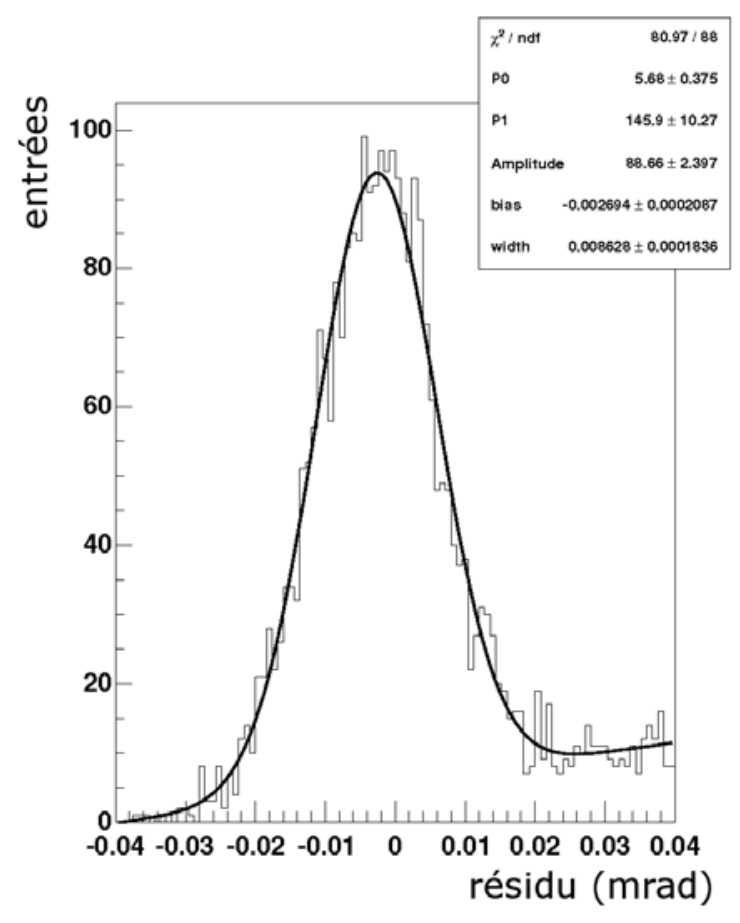

FIG. 3.4: Exemple d'ajustement permettant de déterminer la valeur d'une correction pour une entrée de la table

\subsection{Résultats : Amélioration des performances du DIRC}

Une façon de vérifier l'efficacité de l'alignement consiste à considérer les distributions d'angle de Cherenkov des traces pour chaque barre et de les ajuster par une gaussienne. L'alignement est bon si le biais de la gaussienne est nul et si la résolution, donnée par la largeur de la gaussienne est améliorée.

La figure 3.6 représente la distribution du biais de la distribution de résidus pour chaque barre. Ce graphe permet une comparaison entre l'alignement avant et après correction. Cela montre que le biais est très bien corrigé.

La figure 3.7 représente la distribution de la résolution pour chaque barre. Le graphe montre l'amélioration due à l'alignement. La résolution totale des barres est significativement améliorée car elle passe de 2,81 mrad à 2,54 mrad.

Il est également intéressant de vérifier que le biais est bien corrigé pour tout les angles d'inclinaison d'une trace. La figure 3.8 montre qu'après correction, la distribution du biais en fonction de l'angle d'inclinaison est plate et centrée à 0 .

Les graphes représentant la distribution de biais (Fig. 3.6) et de resolution (Fig. 3.7) par barre permettent également la comparaison entre l'alignement de 2000 et le nouvel alignement. On peut remarquer que l'alignement ne s'est que très peu dégradé. L'impact sur la qualité des données reconstruites utilisant les informations du DIRC sera donc négligeable. Il est cependant rassurant de constater que l'alignement reste bon au cours du temps et d'être capable de le vérifier régulièrement et éventuellement de le recalculer. 


\subsection{Conclusion}

La table de correction ainsi déterminée est alors chargée dans la base de données de BABAR au moment de la reconstruction et est utilisée pour produire les ntuples de données. Le code a été entièrement réorganisé afin de pouvoir recalculer les corrections régulièrement et rapidement. 

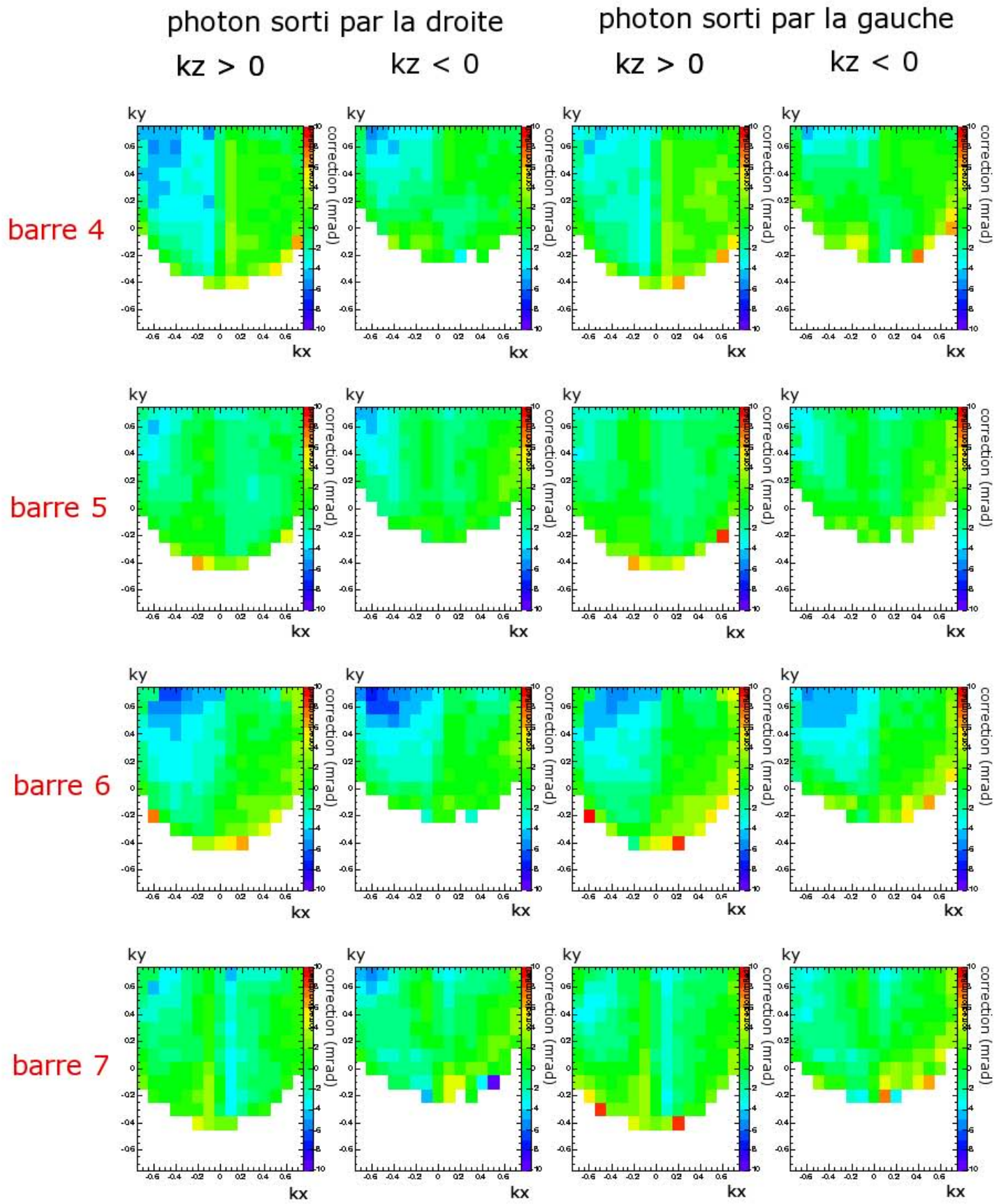

FIG. 3.5: Graphes résumant les valeurs de corrections calculées pour quelques barres. De haut en bas, les graphes correspondent aux barres 4 à 7 . De gauche à droite, les graphes correspondent aux photons qui sortent du prisme par la droite ou par la gauche après s'être ou non réfléchi contre le miroir. 


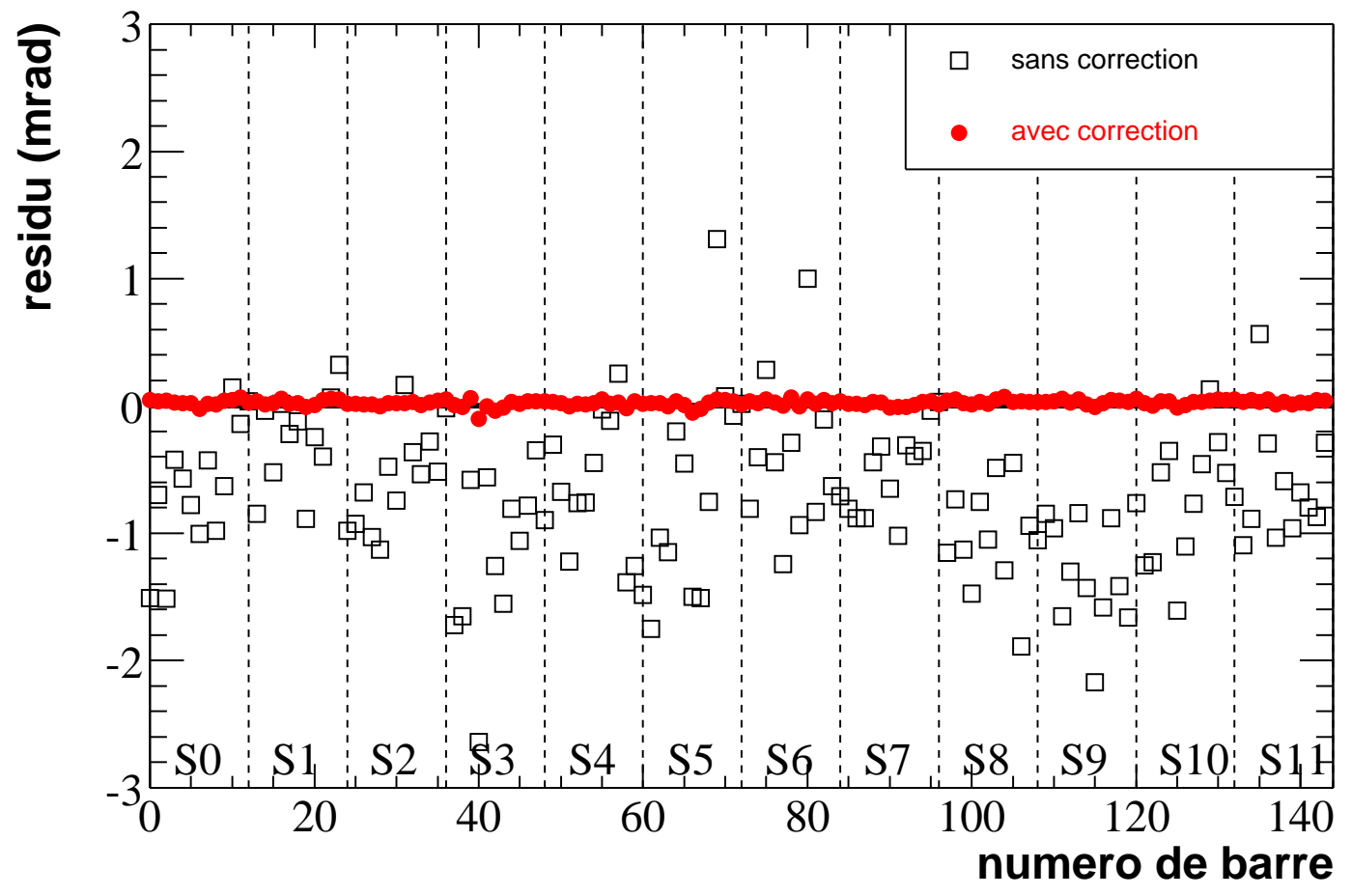

FIG. 3.6: Biais des distributions de résidus de $\theta_{C}$ pour chaque barre, pour les mêmes événements de di-muons, sans et avec correction 


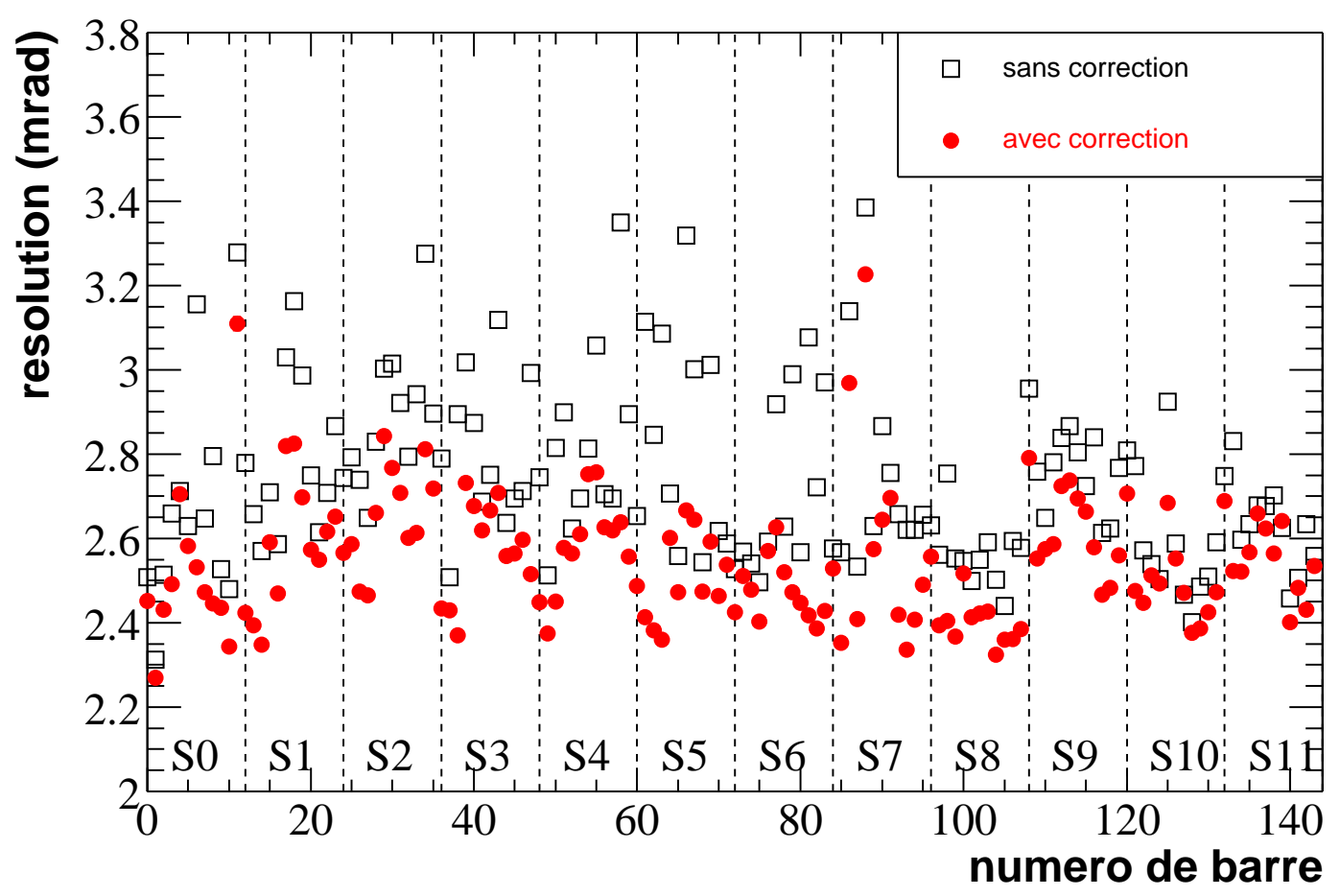

Fig. 3.7: Résolution des distributions de résidus de $\theta_{C}$ pour chaque barre, pour les mêmes événements de di-muons, sans et avec correction 

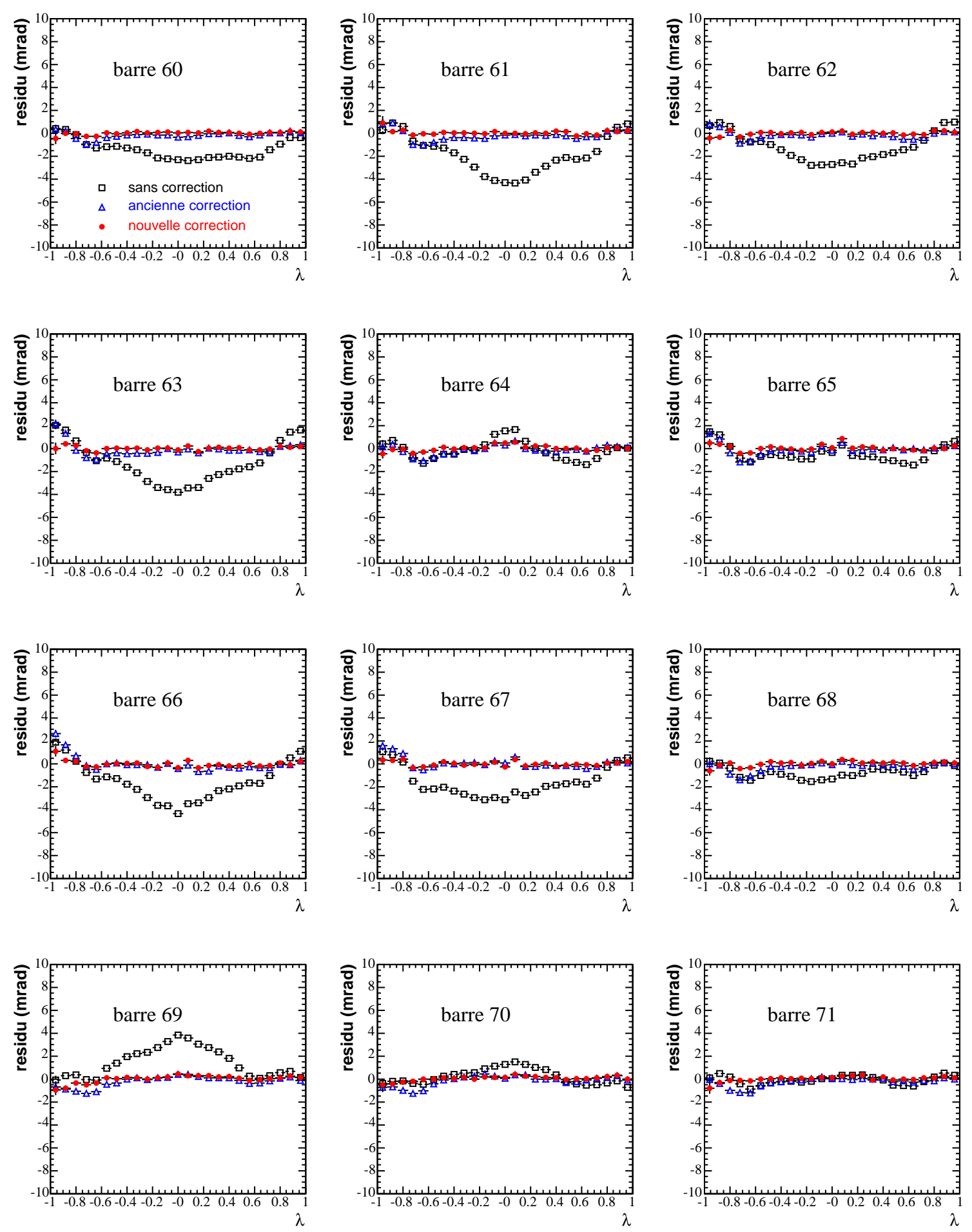

Fig. 3.8: Biais des distributions de résidus de $\theta_{C}$ en fonction de l'angle d'inclinaison pour les barres 60 à 71 . 


\section{La désintégration $B^{0} \rightarrow D^{*-} \pi^{+}$et la mesure de $\sin (2 \beta+\gamma)$}





\section{4 \\ Comment mesurer $\gamma$ ?}

Ce chapitre introduit d'abord brièvement les différentes méthodes pour mesurer $\gamma$, puis décrit comment le canal $B^{0} \rightarrow D^{*-} \pi^{+}$permet de contraindre $\gamma$. En effet, les désintégrations $B^{0} \rightarrow D^{*-} \pi^{+}$permettent l'étude de la violation de $C P$ par la mesure du paramètre $\sin (2 \beta+\gamma)$. L'angle $\beta$ étant maintenant connu avec précision, ce mode permet d'apporter les premières contraintes sur l'angle $\gamma$.

\section{Introduction}

Certaines désintégrations du $B_{s}$ sont particulièrement intéressantes pour mesurer $\gamma$ [50]. Il a été montré que les désintégrations en états finals non états propres de $C P$ pouvaient être très performantes dans certains cas pour mettre en évidence la violation de $C P$ [51]. Par exemple, les modes $B_{s} \rightarrow D_{s}^{(*) \pm} K^{(*) \mp}$ sont les candidats les plus prometteurs pour mesurer $\gamma$ : on s'attend à de grandes asymétries car les amplitudes qui interfèrent sont du même ordre de grandeur et il n'y a pas de pollution par les pingouins. Il est également possible de mesurer $\sin (2 \gamma)$ dans les désintégrations $B_{s} \rightarrow \rho^{0} K_{S}$, dont l'état final est état propre de $C P$. Cependant, les asymétries attendues sont très faibles, et ce mode est de plus pollué par des diagrammes pingouins. Il peut donc y avoir des interactions avec des amplitudes ayant des phases faibles différentes et par conséquent, on ne mesure pas réellement $\gamma$, mais plutôt une phase effective.

Dans les usines à $B$, on ne produit pas de mésons $B_{s}$. Il faudra attendre $L H C b$ pour mesurer $\gamma$ dans ces modes et ce sera d'ailleurs l'un des enjeux principaux de cette expérience. Il est toutefois possible de mesurer $\gamma$ dans BABAR, en utilisant les modes $B^{ \pm} \rightarrow D^{(*) 0} K^{(*) \pm}$. Les asymétries attendues sont importantes, mais les rapports d'embranchement mis en jeux sont très faibles. Une alternative puissante consiste à déduire $\gamma$ de la mesure de $\sin (2 \beta+\gamma)$ en utilisant les désintégrations $B^{0} \rightarrow D^{(*) \mp}\left\{\pi^{ \pm}, \rho^{ \pm}, a_{1}^{ \pm}, \ldots\right\}$, sachant que la mesure de $\sin (2 \beta)$ est maintenant connue avec une très grande précision. Le mode $B^{0} \rightarrow D^{*-} \pi^{+}$sera détaillé plus précisément, car il fait l'objet de cette thèse. Contrairement aux modes $D^{(*)} K^{(*)}$, les asymétries attendues sont très faibles (de l'ordre de quelques \%), mais les rapports d'embranchement sont plus importants. La difficulté de la mesure de $\gamma$ réside dans le fait que l'on a soit des asymétries très petites, soit des rapports d'embranchement très faibles. 


\subsection{Mesure de $\gamma$ avec les modes DK}

\subsubsection{Introduction aux modes $B^{ \pm} \rightarrow D^{0(*)} K^{ \pm(*)}$}

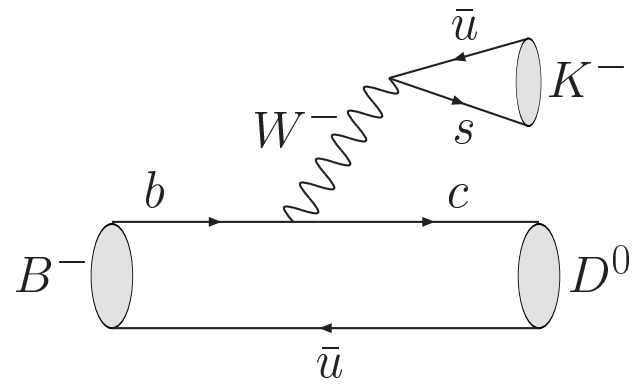

(a)

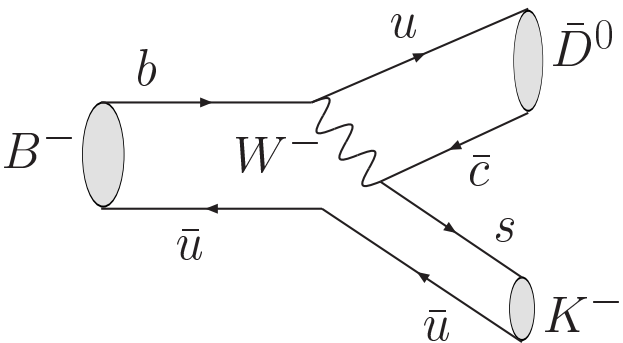

(b)

Fig. 4.1: Diagrammes de Feynman pour les désintégrations $B^{-} \rightarrow D^{0} K^{-}$(a) et $B^{-} \rightarrow \bar{D}^{0} K^{-}$ (b).

Les désintégrations du type $B^{ \pm} \rightarrow D^{0(*)} K^{ \pm(*)}$ (figure 4.1) permettent la mesure directe de l'angle $\gamma$, par l'interférence entre les transitions $b \rightarrow c$ et $b \rightarrow u$ [20]. En effet, la différence de phase faible entre les désintégrations $B^{+} \rightarrow D^{0} K^{+}$et $B^{+} \rightarrow \bar{D}^{0} K^{+}$est proportionnelle à $e^{2 i \gamma}$. Les désintégrations en jeu sont des purs arbres et ne sont pas affectées par de la nouvelle physique éventuelle dans le mixing $B^{0} / \bar{B}^{0}$ ou dans les amplitudes de désintégration. Ces modes sont donc théoriquement très propres.

Pour qu'il y ait interférence, il faut que le $D^{0}$ et le $\bar{D}^{0}$ se désintègrent dans le même état final. À l'origine, une méthode avait été proposée par Gronau et Wyler [52] pour mesurer $\gamma$, dans le cas où le $D^{0}$ se désintègre en un état propre de $C P$, en utilisant les désintégrations $B^{-} \rightarrow D^{0} K^{-}, B^{-} \rightarrow \bar{D}^{0} K^{-}$et $B^{-} \rightarrow D_{ \pm}^{0} K^{-}$, où $D^{0}$ et $\bar{D}^{0}$ représentent les états propres de saveur et $D_{ \pm}^{0}$ les états propres de $C P$ associés aux valeurs propres \pm 1 . Cette méthode, exposée dans la partie 4.1.2, est néanmoins relativement ardue à mettre en oeuvre expérimentalement. Une alternative, qui consiste à considérer les cas dans lesquels le $D^{0}$ ne se désintègre pas en état propre de $C P$, a été proposée par Atwood, Dunietz et Soni (ADS) [53] et sera décrite dans la section 4.1.3. Enfin, une troisième méthode très performante, qui utilise la distribution de Dalitz de la désintégration à trois corps du $D^{0}$ issu du $D^{(*) 0}$, est présentée section ??.

\subsubsection{Méthode de Gronau-London-Wyler (GLW) pour les dés- intégrations $B^{ \pm} \rightarrow D_{ \pm}^{0} K^{ \pm}$}

Pour mettre en oeuvre la méthode GLW, on s'intéresse aux désintégrations $B^{-} \rightarrow$ $D_{ \pm}^{0} K^{-}$, suivie de la désintégration du $D_{ \pm}^{0}$ en état propre de $C P$, comme par exemple $D^{0} \rightarrow \pi^{+} \pi^{-}, K^{+} K^{-}, \ldots\left(\eta_{C P}=1\right)$ ou $D^{0} \rightarrow \pi^{0} K_{S}, \ldots\left(\eta_{C P}=-1\right)$. De même que pour les $B^{0}$, on peut écrire une relation entre les états propres de saveur et les états propres de $C P$ du $D^{0}$. On choisit ici une convention de phase telle que : 


$$
\left|D_{ \pm}^{0}\right\rangle=\frac{1}{\sqrt{2}}\left(\left|D^{0}\right\rangle \pm\left|\bar{D}^{0}\right\rangle\right)
$$

On en déduit les relations suivantes entre les amplitudes de transitions $B^{ \pm} \rightarrow D_{+}^{0} K^{ \pm}$:

$$
\begin{aligned}
& \mathcal{A}\left(B^{+} \rightarrow D_{+}^{0} K^{+}\right)=\frac{1}{\sqrt{2}}\left(\mathcal{A}\left(B^{+} \rightarrow D^{0} K^{+}\right)+\mathcal{A}\left(B^{+} \rightarrow \bar{D}^{0} K^{+}\right)\right), \\
& \mathcal{A}\left(B^{-} \rightarrow D_{+}^{0} K^{-}\right)=\frac{1}{\sqrt{2}}\left(\mathcal{A}\left(B^{-} \rightarrow D^{0} K^{-}\right)+\mathcal{A}\left(B^{-} \rightarrow \bar{D}^{0} K^{-}\right)\right) .
\end{aligned}
$$

Ces relations peuvent être représentées sous la forme de triangles dans le plan complexe. La phase $\gamma$ vient de l'interférence entre les diagrammes de la figure 4.1. La désintégration $B^{-} \rightarrow D K^{-}$peut en effet se faire via les transitions $b \rightarrow u$ ou $b \rightarrow c$. On peut donc écrire :

$$
\begin{aligned}
& \mathcal{A}\left(B^{+} \rightarrow D^{0} K^{+}\right)=e^{i \gamma} \lambda\left|V_{c b}\right| R_{b}|a| e^{i \Delta_{a}}=e^{2 i \gamma} \mathcal{A}\left(B^{-} \rightarrow \bar{D}^{0} K^{-}\right) \\
& \mathcal{A}\left(B^{+} \rightarrow \bar{D}^{0} K^{+}\right)=\lambda\left|V_{c b}\right||A| e^{i \Delta_{A}}=\mathcal{A}\left(B^{-} \rightarrow D^{0} K^{-}\right)
\end{aligned}
$$

où $\lambda R_{b}=\left(1-\lambda^{2} / 2\right)\left|V_{u b} / V_{c b}\right|$.

Ces rapports d'embranchement sont reliés à $\gamma$, comme on le voit sur la figure 4.2.

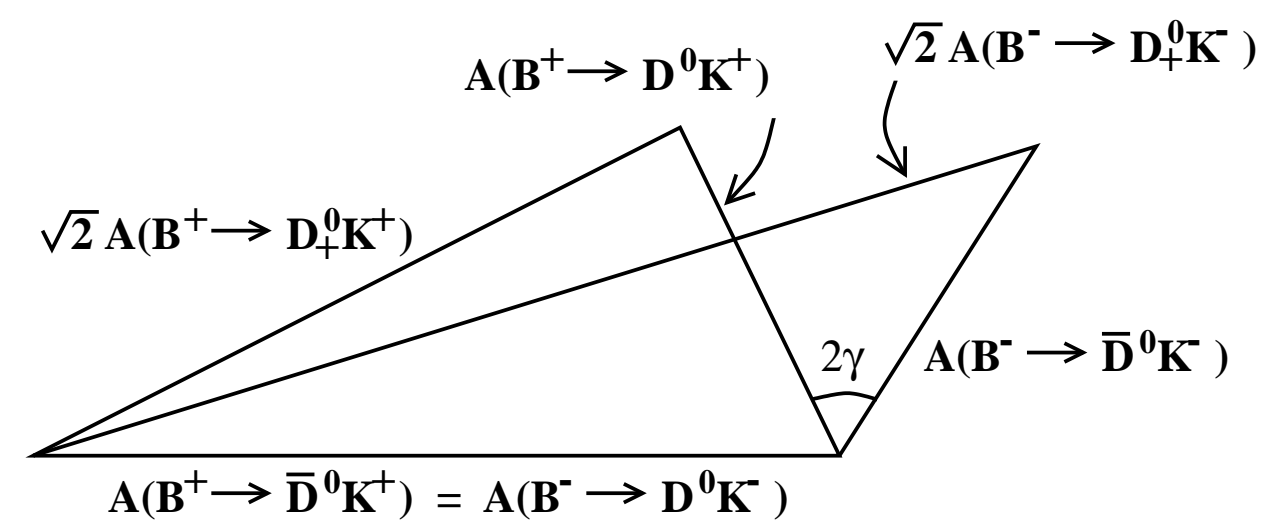

FIG. 4.2: Relations triangulaires entre les différentes amplitudes de transition du type $B^{ \pm} \rightarrow$ $D K^{ \pm}$

Le problème est qu'en réalité, ces triangles sont très aplatis car $B^{-} \rightarrow \bar{D}^{0} K^{-}$est à la fois supprimé de couleur et de CKM par rapport à $B^{-} \rightarrow D^{0} K^{-}$. On ne mesure donc pas les différentes amplitudes. En pratique, on a accès aux observables $R_{(C P)}$ qui sont les rapports des amplitudes supprimées de Cabibbo et favorisées de Cabibbo, avec $D^{0}$ qui se désintègre en état propre de $C P\left(R_{C P}\right)$ ou en état favorisé de Cabibbo $(R) . R_{(C P)}$ s'exprime de la façon suivante :

$$
R_{(C P)}=\frac{\mathcal{B}\left(B^{-} \rightarrow D^{0}\left(D_{C P}^{0}\right) K^{-}\right)+\mathcal{B}\left(B^{+} \rightarrow \bar{D}^{0}\left(D_{C P}^{0}\right) K^{+}\right)}{\mathcal{B}\left(B^{-} \rightarrow D^{0}\left(D_{C P}^{0}\right) \pi^{-}\right)+\mathcal{B}\left(B^{+} \rightarrow \bar{D}^{0}\left(D_{C P}^{0}\right) \pi^{+}\right)}
$$


Le rapport $R_{C P} / R$ est directement relié à $\gamma$ dans le cadre du Modèle Standard :

$$
\frac{R_{C P}}{R}=1+r_{B}^{2}+2 r_{B} \cos \delta \cos \gamma,
$$

où $r_{B}$ est le rapport entre l'amplitude de la désintégration $B^{-} \rightarrow \bar{D}^{0} K^{-}$et l'amplitude de la désintégration $B^{-} \rightarrow D^{0} K^{-}$et $\delta$ est la phase forte relative (inconnue) entre ces deux amplitudes.

On peut également mesurer une asymétrie de $C P$ définie ainsi :

$$
A_{C P}=\frac{\mathcal{B}\left(B^{-} \rightarrow D_{C P}^{0} K^{-}\right)-\mathcal{B}\left(B^{+} \rightarrow D_{C P}^{0} K^{+}\right)}{\mathcal{B}\left(B^{-} \rightarrow D_{C P}^{0} K^{-}\right)+\mathcal{B}\left(B^{+} \rightarrow D_{C P}^{0} K^{+}\right)}
$$

Cette asymétrie est reliée à $\gamma$ ainsi :

$$
A_{C P}=2 r_{B} \sin \delta \sin \gamma /\left(1+r_{B}^{2}+2 r_{B} \cos \delta \cos \gamma\right) .
$$

Les résultats des analyses de BABAR (Belle) sont résumés dans le tableau 4.1 (4.2).

\begin{tabular}{|c|c|c|}
\hline mode & $R_{C P \pm} / R$ & $A_{C P \pm}$ \\
\hline$B^{ \pm} \rightarrow D^{0} K^{ \pm}[54]$ & $R_{C P+} / R=0,87 \pm 0,14 \pm 0,06$ & $A_{C P+}=0,40 \pm 0,15 \pm 0,08$ \\
$\mathcal{L}=194 f b^{-1}$ & $R_{C P-} / R=0,80 \pm 0,14 \pm 0,08$ & $A_{C P-}=0,21 \pm 0,17 \pm 0,07$ \\
\hline$B^{ \pm} \rightarrow D^{0} K^{* \pm}[55]$ & $R_{C P+} / R=1,73 \pm 0,36 \pm 0,11$ & $A_{C P+}=-0,08 \pm 0,20 \pm 0,06$ \\
$\mathcal{L}=206 f b^{-1}$ & $R_{C P-} / R=0,64 \pm 0,25 \pm 0,07$ & $A_{C P-}=-0,35 \pm 0,38 \pm 0,10$ \\
\hline$B^{ \pm} \rightarrow D^{* 0} K^{ \pm}[56]$ & $R_{C P+} / R=1,06 \pm 0,26 \pm 0,10$ & $A_{C P+}=-0,10 \pm 0,23 \pm 0,04$ \\
\cline { 2 - 3 } $\mathcal{L}=112 f b^{-1}$ & & \\
\hline
\end{tabular}

TAB. 4.1: Résumé des mesures de $R_{C P \pm}$ et de $A_{C P \pm}$ pour les modes $B^{ \pm} \rightarrow D^{(*) 0} K^{(*) \pm}$ par l'expérience BABAR.

\begin{tabular}{|c|c|c|}
\hline mode & $R_{C P \pm} / R$ & \multicolumn{1}{|c|}{$A_{C P \pm}$} \\
\hline$B^{ \pm} \rightarrow D^{0} K^{ \pm}[57]$ & $R_{C P+} / R=0,98 \pm 0,18 \pm 0,10$ & $A_{C P+}=0,07 \pm 0,14 \pm 0,06$ \\
$\mathcal{L}=194 f b^{-1}$ & $R_{C P-} / R=1,29 \pm 0,16 \pm 0,08$ & $A_{C P-}=-0,11 \pm 0,14 \pm 0,05$ \\
\hline$B^{ \pm} \rightarrow D^{* 0} K^{ \pm}[57]$ & $R_{C P+} / R=1,43 \pm 0,28 \pm 0,06$ & $A_{C P+}=-0,27 \pm 0,25 \pm 0,04$ \\
$\mathcal{L}=112 f b^{-1}$ & $R_{C P-} / R=0,94 \pm 0,28 \pm 0,06$ & $A_{C P-}=0,26 \pm 0,26 \pm 0,03$ \\
\hline
\end{tabular}

TAB. 4.2: Résumé des mesures de $R_{C P \pm}$ et de $A_{C P \pm}$ pour les modes $B^{ \pm} \rightarrow D^{(*) 0} K^{ \pm}$par l'expérience Belle.

Les rapports $R_{C P \pm} / R$ sont compatibles avec 1 et les asymétries $A_{C P \pm}$ sont compatibles avec 0 . Avec la statistique actuelle, les contraintes sur $\gamma$ apportées par ce type d'analyse sont très faibles.

\subsubsection{Méthode de Atwood-Dunietz-Soni (ADS) pour les désin- tégrations $B^{ \pm} \rightarrow D^{0} K^{ \pm}$}

Cette méthode s'inspire de la méthode GLW et a pour but de pallier le problème de la forte suppression d'un diagramme par rapport à l'autre. L'idée est de considérer la dés- 
intégration favorisée $B^{-} \rightarrow D^{0} K^{-}$suivie d'une désintégration doublement supprimée de Cabibbo du $D^{0}$, et la désintégration défavorisée $B^{-} \rightarrow \bar{D}^{0} K^{-}$suivie d'une désintégration favorisée de Cabibbo du $\bar{D}^{0}$. Ainsi on s'attend au total à de larges interférences. Dans ce cas-là, on ne considère pas un $D^{0}$ qui se désintègre en état propre de $C P$. Des exemples de désintégration du $D^{0}$ sont $K^{+} \pi^{-}, K^{+} \pi^{-} \pi^{0} \ldots$

L'observable utilisée $R$ est le rapport entre les rapports d'embranchements des transitions supprimées et favorisées :

$$
R=\frac{\mathcal{B}\left(B^{-} \rightarrow D_{K^{+} \pi^{-}} K^{-}\right)-\mathcal{B}\left(B^{+} \rightarrow D_{K^{-} \pi^{+}} K^{+}\right)}{\mathcal{B}\left(B^{-} \rightarrow D_{K^{-} \pi^{+}} K^{-}\right)+\mathcal{B}\left(B^{+} \rightarrow D_{K^{+} \pi^{-}} K^{+}\right)}
$$

$R$ est relié à $\gamma$ de la façon suivante :

$$
R=r_{D}^{2}+r_{B}^{2}+2 r_{D} r_{B} \cos (\gamma) \cos (\delta),
$$

où $r_{B}$ a la même signification que précédemment et $r_{D}$ est le rapport entre l'amplitude $\bar{D}^{0} \rightarrow K^{+} \pi^{-}$et l'amplitude $D^{0} \rightarrow K^{+} \pi^{-}$. Une analyse de BABAR utilisant une luminosité intégrée de $205 \mathrm{fb}^{-1}$ a permis de mettre la limite $r_{B}<0,23$ à $90 \%$ de niveau de confiance [58]. Une analyse de Belle utilisant une luminosité intégrée de $250 \mathrm{fb}^{-1}$ a permis de mettre la limite $r_{B}<0,27$ à $90 \%$ de niveau de confiance [59]. Les mesures actuelles prédisent une valeur de $r_{B}$ très faible, ce qui laisse à penser qu'il sera difficile de mesurer $\gamma$ de cette façon-là.

\subsubsection{Mesure de $\gamma$ dans les désintégrations $B^{ \pm} \rightarrow D^{(*) 0} K^{ \pm}$avec une analyse de Dalitz de la désintégration du $D^{(*) 0}$}

Cette méthode prometteuse utilise les modes $B^{-} \rightarrow D^{(*) 0} K^{-}$avec le $D^{(*) 0}$ qui se désintègre en en trois corps, par exemple en $K_{s} \pi^{+} \pi^{-}$. On réalise alors une analyse de la distribution de Dalitz de la désintégration du $D^{(*) 0}$. [60]. L'avantage de cette méthode est qu'elle met en jeu toute la structure résonnante de la désintégration à trois corps (en particulier la résonance $K^{*}$ ), avec des amplitudes supprimées et favorisées de Cabibbo qui interfèrent directement.

L'amplitude de désintégration de $B^{\mp}$ est :

$$
\mathcal{A}_{\mp}=\mathcal{A}_{D}\left(m_{\mp}^{2}, m_{ \pm}^{2}\right)+r_{B}^{(*)} e^{i\left(\delta_{B}^{(*)} \mp \gamma\right)} \mathcal{A}_{D}\left(m_{ \pm}^{2}, m_{\mp}^{2}\right),
$$

où $m_{ \pm}^{2}$ et $m_{\mp}^{2}$ sont respectivement les masses invariantes des combinaisons $K_{s}^{0} \pi^{-}$et $K_{s}^{0} \pi^{+}$et $\mathcal{A}_{D}\left(m_{\mp}^{2}, m_{ \pm}^{2}\right)$ est l'amplitude de désintégration du $D^{(*) 0} \cdot r_{B}^{(*)}$ et $\delta_{B}^{(*)}$ représentent respectivement le rapport d'amplitude et la phase forte relative entre l'amplitude supprimée et l'amplitude favorisée de la désintégration du $B^{ \pm}$.

$\mathcal{A}_{D}\left(m_{\mp}^{2}, m_{ \pm}^{2}\right)$ peut être déterminée par l'analyse de Dalitz d'un échantillon de $D^{0}$ étiquetés, provenant de la désintégration de $D^{*+}$ en $D^{0} \pi$.

Cette méthode a été utilisée par les expériences BABAR et Belle. L'analyse de BABAR [61], réalisée avec une luminosité intégrée de $205 \mathrm{fb}^{-1}$, a mesuré $r_{B}=0,118 \pm 0,079 \pm$ $0,034_{-0,034}^{+0,036}, r_{B}^{*}=0,169 \pm 0,096_{-0,028-0,026}^{+0,030+0,029}$ et $\gamma=\left(70 \pm 31_{-10-11}^{+12+14}\right)^{\circ}$. L'analyse de Belle [62], réalisée avec une luminosité intégrée de $253 \mathrm{fb}^{-1}$, a mesuré $\gamma=\left(68_{-15}^{+14} \pm 13 \pm 11\right)^{\circ}$. Les 
deux expérience ont donc des mesures parfaitement compatibles. La mesure de Belle est celle qui fournit la meilleure contrainte sur $\gamma$ à l'heure actuelle, mais dépend de modèles théoriques. La grande précision de la mesure de Belle s'explique par le fait que le rapport $r_{B}^{(*)}$ mesuré dans cette analyse est très grand.

\subsection{Le canal $B \rightarrow D^{* \mp} \pi^{ \pm}$et la mesure de $\sin (2 \beta+\gamma)$}

\subsubsection{Introduction}

Les diagrammes de Feynman correspondant à cette désintégration sont représentés Fig. 4.3.

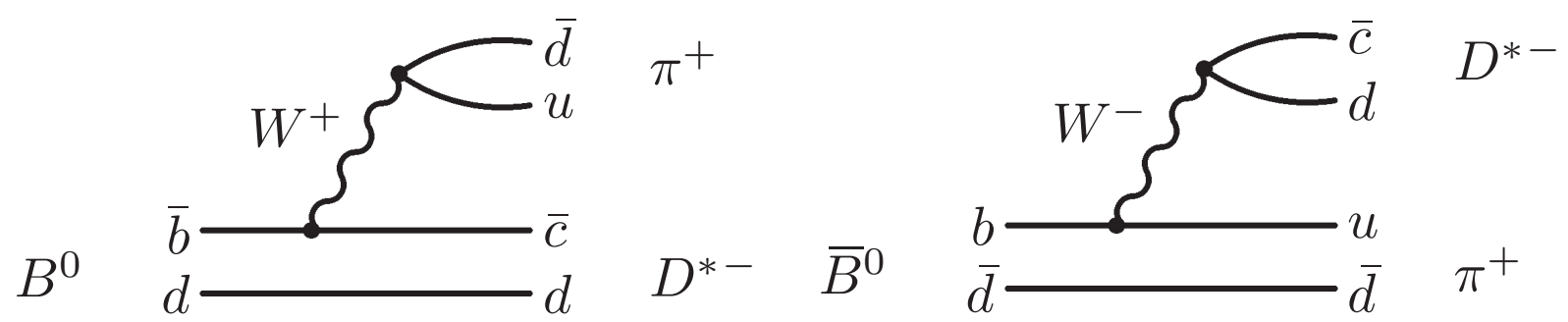

Fig. 4.3: Diagrammes de Feynman pour $B^{0} \rightarrow D^{*} \pi$

Dans ce canal de désintégration, l'état final n'est pas un état propre de $C P$, mais est néanmoins accessible aux désintégrations du $B^{0}$ et du $\bar{B}^{0}$. On peut donc envisager un effet de violation de $C P$ dans l'interférence entre le mélange et la désintégration. $B^{0}$ peut se désintégrer directement en $D^{*-} \pi^{+}\left(B^{0} \rightarrow D^{*-} \pi^{+}\right)$ou après s'être mélangé en $\bar{B}^{0}$ $\left(B^{0} \rightarrow \bar{B}^{0} \rightarrow D^{*-} \pi^{+}\right)$. La désintégration peut donc se faire par une transition $\bar{b} \rightarrow \bar{c}(u \bar{d})$ (diagramme de gauche) ou par une transition $\bar{b} \rightarrow b \rightarrow u(\bar{c} d)$ (diagramme de droite). Ce dernier est supprimé par rapport au premier d'un facteur $\lambda^{2}$.

On considère l'interférence entre la désintégration favorisée $B^{0} \rightarrow D^{*-} \pi^{+}$, dont l'amplitude est proportionnelle aux éléments de la matrice de CKM $V_{c b}^{*} V_{u d}$, et la désintégration défavorisée $\bar{B}^{0} \rightarrow D^{*-} \pi^{+}$, dont l'amplitude est proportionnelle aux éléments de la matrice de CKM $V_{c d} V_{u b}^{*}$. La phase faible relative entre ces deux amplitudes est donc $\gamma$. Il faut également prendre en compte une phase faible $2 \beta$ qui provient du mélange $B^{0} \bar{B}^{0}$. La phase faible totale que l'on peut mesurer grâce à ce mode est donc $2 \beta+\gamma$ [63-65].

Dans ce canal, il n'y a pas de pollution par les pingouins : l'interprétation de l'asymétrie est donc théoriquement non ambiguë. Il existe également un diagramme d'échange, mais il est très supprimé par rapport au diagramme en arbre. La difficulté réside dans l'amplitude des interférences, qui est très faible en raison de la valeur du rapport $r^{*}$ :

$$
r^{*}=\left|\frac{\mathcal{A}\left(B^{0} \rightarrow D^{*-} \pi^{+}\right)}{\mathcal{A}\left(\bar{B}^{0} \rightarrow D^{*-} \pi^{+}\right)}\right| \approx 0,02 .
$$

Les asymétries provenant de l'interférence entre ces deux diagrammes sont proportionnelles à $2 r^{*}$ et on s'attend donc à ce qu'elles soient petites. Cependant le rapport d'embran- 
chement de $B^{0} \rightarrow D^{*-} \pi^{+}$est relativement élevé $: \mathcal{B}\left(B^{0} \rightarrow D^{*-} \pi^{+}\right)=(2.76 \pm 0.21) \times 10^{-3}$ [29].

Notons qu'il y a deux désintégrations possibles : $B^{0} \rightarrow D^{*+} \pi^{-}$et $B^{0} \rightarrow D^{*-} \pi^{+}$. A priori, chaque mode de désintégration devrait donner une mesure indépendante de $\sin (2 \beta+\gamma)$, mais dans l'analyse, on utilise les deux simultanément.

\subsection{2 Évolution temporelle de la désintégration $B \rightarrow D^{* \mp} \pi^{ \pm}$}

Ainsi que cela a été expliqué dans les chapitres précédents, l'évolution temporelle de la désintégration $B \rightarrow D^{* \mp} \pi^{ \pm}$dépend de la différence de temps $\Delta t$ entre les désintégrations des deux $B$ produits par la désintégration de la résonance $\Upsilon(4 S)$. On reprend les notations

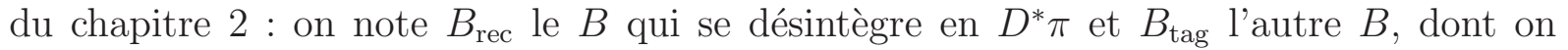
étiquette la saveur grâce à la reconstruction exclusive de ses produits de désintégration. Cette partie décrit l'évolution temporelle du côté de $B_{\text {rec }}$ uniquement.

La désintégration $B \rightarrow D^{* \mp} \pi^{ \pm}$est décrite par quatre équations d'évolution temporelle, selon l'état initial $B^{0}$ ou $\bar{B}^{0}$ et l'état final $D^{*-} \pi^{+}$ou $D^{*+} \pi^{-}$.

Les amplitudes des désintégrations avec la transition de quark $b \rightarrow c$ peuvent s'écrire [66] :

$$
\begin{aligned}
& \left\langle D^{*-} \pi^{+}|\mathcal{T}| B^{0}\right\rangle=A e^{i \delta_{c}}, \\
& \left\langle D^{*+} \pi^{-}|\mathcal{T}| \bar{B}^{0}\right\rangle=A e^{i \delta_{c}},
\end{aligned}
$$

où $A$ et $\delta_{c}$ représentent l'amplitude et la phase forte. Dans la convention de Wolfenstein, la phase faible est nulle.

Les amplitudes des désintégrations avec la transition de quark $b \rightarrow u$ peuvent s'écrire [66] :

$$
\begin{aligned}
\left\langle D^{*-} \pi^{+}|\mathcal{T}| \bar{B}^{0}\right\rangle & =a e^{i \delta_{u}} e^{-i \gamma} \\
\left\langle D^{*+} \pi^{-}|\mathcal{T}| B^{0}\right\rangle & =a e^{i \delta_{u}} e^{+i \gamma}
\end{aligned}
$$

où $a$ est l'amplitude, $\delta_{u}$ la phase forte et $\gamma$ la phase faible. On remarque que le signe de la phase faible change sous l'action de $C P$, alors que le signe de la phase forte est conservé.

D'autre part, avec l'approximation $|q / p|=1$, on a $q / p=e^{-2 i \beta}$ dans le cadre de la convention de phase de Wolfenstein.

Avec les notations de l'équation 1.23, on obtient [65] :

$$
\lambda_{D^{*-} \pi^{+}}=r^{*} e^{-i(2 \beta+\gamma)} e^{i \delta}, \quad \lambda_{D^{*+} \pi^{-}}=r^{*} e^{i(2 \beta+\gamma)} e^{i \delta},
$$

où $\delta$ représente la différence de phase forte entre les deux amplitudes $(b \rightarrow c$ et $b \rightarrow u$ ) et $r^{*}=a / A$.

On déduit les évolutions temporelles, des équations 1.24 et 1.25 : 


$$
\begin{aligned}
\left|\left\langle D^{*-} \pi^{+}|\mathcal{T}| B^{0}\right\rangle\right|^{2}= & \frac{e^{-\Gamma|\Delta t|}}{2} A^{2}[(1+\cos (\Delta m \Delta t)) \\
& +(1-\cos (\Delta m \Delta t)) r^{* 2} \\
& \left.+2 r^{*} \sin (2 \beta+\gamma-\delta) \sin (\Delta m \Delta t)\right] \\
\left|\left\langle D^{*-} \pi^{+}|\mathcal{T}| \bar{B}^{0}\right\rangle\right|^{2}= & \frac{e^{-\Gamma|\Delta t|}}{2} A^{2}[(1-\cos (\Delta m \Delta t)) \\
& +(1+\cos (\Delta m \Delta t)) r^{* 2} \\
& \left.-2 r^{*} \sin (2 \beta+\gamma-\delta) \sin (\Delta m \Delta t)\right] \\
\left|\left\langle D^{*+} \pi^{-}|\mathcal{T}| B^{0}\right\rangle\right|^{2}= & \frac{e^{-\Gamma|\Delta t|}}{2} A^{2}[(1-\cos (\Delta m \Delta t)) \\
& +(1+\cos (\Delta m \Delta t)) r^{* 2} \\
& \left.+2 r^{*} \sin (2 \beta+\gamma+\delta) \sin (\Delta m \Delta t)\right] \\
\left|\left\langle D^{*+} \pi^{-}|\mathcal{T}| \bar{B}^{0}\right\rangle\right|^{2}= & \frac{e^{-\Gamma|\Delta t|}}{2} A^{2}[(1+\cos (\Delta m \Delta t)) \\
& +(1-\cos (\Delta m \Delta t)) r^{* 2} \\
& \left.-2 r^{*} \sin (2 \beta+\gamma+\delta) \sin (\Delta m \Delta t)\right]
\end{aligned}
$$

Ces équations peuvent être réécrites de la façon suivante :

$$
\begin{aligned}
2 \frac{\left|\left\langle D^{*-} \pi^{+}|\mathcal{T}| B^{0}\right\rangle\right|^{2}}{A^{2}+a^{2}}= & e^{-\Gamma|\Delta t|}[1+C \cos (\Delta m \Delta t) \\
& +S \sin (2 \beta+\gamma-\delta) \sin (\Delta m \Delta t)], \\
2 \frac{\left|\left\langle D^{*-} \pi^{+}|\mathcal{T}| \bar{B}^{0}\right\rangle\right|^{2}}{A^{2}+a^{2}}= & e^{-\Gamma|\Delta t|}[1-C \cos (\Delta m \Delta t) \\
& -S \sin (2 \beta+\gamma-\delta) \sin (\Delta m \Delta t)], \\
2 \frac{\left|\left\langle D^{*+} \pi^{-}|\mathcal{T}| B^{0}\right\rangle\right|^{2}}{A^{2}+a^{2}}= & e^{-\Gamma|\Delta t|}[1-C \cos (\Delta m \Delta t) \\
& +S \sin (2 \beta+\gamma+\delta) \sin (\Delta m \Delta t)], \\
2 \frac{\left|\left\langle D^{*+} \pi^{-}|\mathcal{T}| \bar{B}^{0}\right\rangle\right|^{2}}{A^{2}+a^{2}}= & e^{-\Gamma|\Delta t|}[(1+C \cos (\Delta m \Delta t) \\
& -S \sin (2 \beta+\gamma+\delta) \sin (\Delta m \Delta t)],
\end{aligned}
$$

avec $C=\left(1-r^{* 2}\right) /\left(1+r^{* 2}\right)$ et $S=2 r^{*} /\left(1+r^{* 2}\right)$.

Une analyse dépendant du temps de $B^{0} \rightarrow D^{*-} \pi^{+}$permet en principe d'extraire les paramètres $r^{*}, \sin (2 \beta+\gamma+\delta)$ et $\sin (2 \beta+\gamma-\delta)$. Cependant, en pratique on n'a pas assez de statistique pour mesurer $r^{*}$. En négligeant les termes en $r^{* 2}$, on a $C=1$ et $S=2 r^{*}$ et les paramètres auxquels on a accès expérimentalement sont $2 r^{*} \times \sin (2 \beta+\gamma+\delta)$ et $2 r^{*} \times \sin (2 \beta+\gamma-\delta)$. 


\section{Impact des interférences du côté du $B_{\text {tag }}$ sur l'évolution temporelle}

La saveur de $B_{\text {tag }}$ peut-être étiquetée par un kaon issu de la désintégration du $B_{\text {tag. }}$ La transition du quark $b$ favorisée est la transition $b \rightarrow c \bar{u} d$. Le quark $s$ du $K$ est alors produit par la transition favorisée $c \rightarrow s$. Il est possible cependant qu'il y ait des interférences entre des transitions favorisées de Cabibbo du type $b \rightarrow c \bar{u} d$ et des transitions supprimées de Cabibbo du type $\bar{b} \rightarrow \bar{u} c \bar{d}$ dans les états finals du $B_{\text {tag. }}$. Ces interférences sont de l'ordre de $2 \%$ [67]. Cet effet d'interférence du côté du $B_{\text {tag }}$, qui est présent dans toutes les analyses de violation de $C P$ dépendant du temps, est généralement négligé car il est très faible par rapport aux asymétries considérées. Cependant, dans le cas de la désintégration $B^{0} \rightarrow D^{*-} \pi^{+}$, les asymétries de $C P$ attendues sont du même ordre de grandeur que les interférences possibles du côté de $B_{\text {tag. }}$. Il est donc nécessaire d'en tenir compte dans la paramétrisation de l'évolution temporelle en $\Delta t$. On introduit des paramètres effectifs $r^{\prime}$ et $\delta^{\prime}$, qui ont la même signification physique que les paramètres $r^{*}$ et $\delta$ utilisés pour décrire la violation de $C P$ du côté de $B_{\text {rec }}$, pour décrire les interférences du côté du $B_{\text {tag }}$. Ces paramètres sont effectifs car plusieurs états finals du $B_{\operatorname{tag}}$ sont possibles. $r^{\prime}\left(\delta^{\prime}\right)$ est le rapport effectif des amplitudes (la différence de phase forte) entre $b \rightarrow c \bar{u} d$ et $\bar{b} \rightarrow \bar{u} c \bar{d}$ pour le $B_{\text {tag. }}$ En tenant compte de cet effet, les équations s'écrivent :

$$
\begin{aligned}
2 \frac{\left|\left\langle D^{*-} \pi^{+}|\mathcal{T}| B^{0}\right\rangle\right|^{2}}{A^{2}+a^{2}}= & e^{-\Gamma|\Delta t|}[1+\cos (\Delta m \Delta t) \\
& +(a-b-c) \sin (\Delta m \Delta t)], \\
2 \frac{\left|\left\langle D^{*-} \pi^{+}|\mathcal{T}| \bar{B}^{0}\right\rangle\right|^{2}}{A^{2}+a^{2}}= & e^{-\Gamma|\Delta t|}[1-\cos (\Delta m \Delta t) \\
& +(-a+b+c) \sin (\Delta m \Delta t)], \\
2 \frac{\left|\left\langle D^{*+} \pi^{-}|\mathcal{T}| B^{0}\right\rangle\right|^{2}}{A^{2}+a^{2}}= & e^{-\Gamma|\Delta t|}[1-\cos (\Delta m \Delta t) \\
& +(a-b+c) \sin (\Delta m \Delta t)], \\
2 \frac{\left|\left\langle D^{*+} \pi^{-}|\mathcal{T}| \bar{B}^{0}\right\rangle\right|^{2}}{A^{2}+a^{2}}= & e^{-\Gamma|\Delta t|}[(1+\cos (\Delta m \Delta t) \\
& +(-a+b-c) \sin (\Delta m \Delta t)],
\end{aligned}
$$

où

$$
\begin{aligned}
& a=2 r^{*} \sin (2 \beta+\gamma) \cos \delta \\
& b=2 r^{\prime} \sin (2 \beta+\gamma) \cos \delta^{\prime}, \\
& c=2 \cos (2 \beta+\gamma)\left(r^{*} \sin \delta-r^{\prime} \sin \delta^{\prime}\right) .
\end{aligned}
$$

La figure 4.4 illustre l'impact que peut avoir un $r^{\prime}$ différent de 0 sur la distribution temporelle d'événements mélangés. 


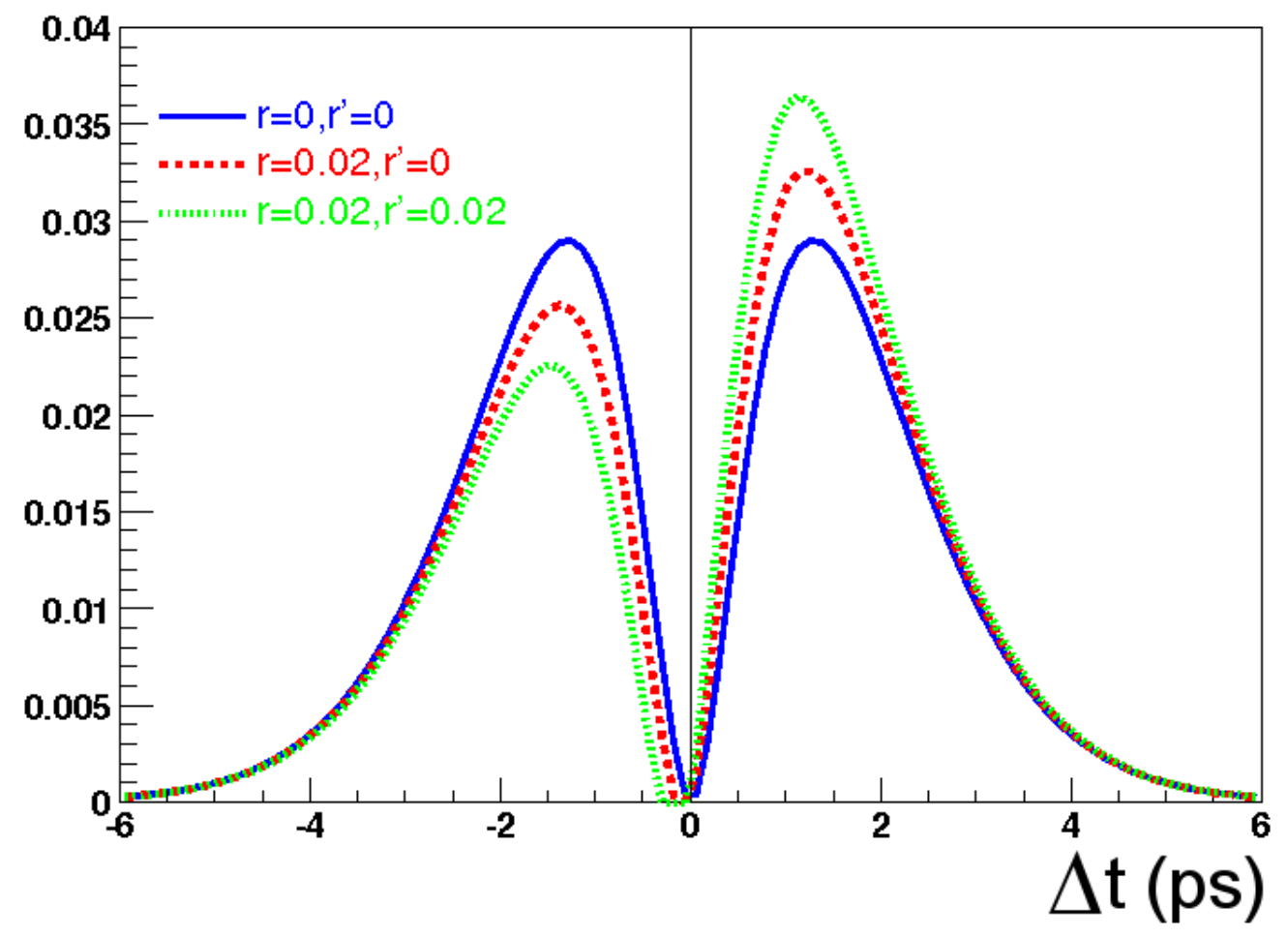

FIG. 4.4: Distribution dépendant du temps pour des événements mélangés avec un $B_{\text {rec }}$ qui se désintègre en $D^{*} \pi$ et un $B_{\text {tag }}$ qui est un $B^{0}$, étiqueté par un kaon. Ici, on considère le cas idéal où l'étiquetage est parfait et où il n'y a pas d'effet de résolution. La courbe en trait plein correspond au cas $r^{*}=r^{\prime}=0$, la courbe en tiretés au cas $r^{*}=0.02$ et $r^{\prime}=0$ et la courbe en pointillés au cas $r^{*}=r^{\prime}=0$. 
Cette paramétrisation est utilisée dans la suite. Notons que dans le cas des événements étiquetés par un lepton, on considère qu'il n'y a pas de violation de $C P$ possible du côté de $B_{\text {tag }}$ car le rapport $b \rightarrow l^{+} / b \rightarrow l^{-}$est négligeable. On a donc $r^{\prime}=0$. Les paramètres de $C P$ sont alors $a=2 r^{*} \sin (2 \beta+\gamma) \cos \delta, b=0$ et $c=2 r^{*} \cos (2 \beta+\gamma) \sin \delta$.

\subsubsection{Détermination du rapport $r^{*}$}

Il n'est pas possible de mesurer $r^{*}$ directement dans les données car on n'a pas assez de statistique à l'heure actuelle pour mesurer $\mathcal{B}\left(B^{0} \rightarrow D^{*+} \pi^{-}\right)$ou $\mathcal{B}\left(\bar{B}^{0} \rightarrow D^{*-} \pi^{+}\right)$. Afin de déterminer $r^{*}$, on utilise la mesure $\mathcal{B}\left(B^{0} \rightarrow D_{s}^{*+} \pi^{-}\right)$. En suppose la symétrie $S U(3)$ de saveur, on a la relation $[64,65]$ :

$$
r^{*}=\tan \left(\theta_{C}\right) \sqrt{\frac{\mathcal{B}\left(B^{0} \rightarrow D_{s}^{*+} \pi^{-}\right) \mathcal{B}\left(D_{s}^{+} \rightarrow \phi \pi^{+}\right)}{\mathcal{B}\left(B^{0} \rightarrow D^{*-} \pi^{+}\right) \mathcal{B}\left(D_{s}^{+} \rightarrow \phi \pi^{+}\right)}} \frac{f_{D^{*}}}{f_{D_{s}^{*}}} .
$$

où $\theta_{C}$ est l'angle de Cabibbo et où $f_{D^{*}}$ et $f_{D_{s}^{*}}$ sont les facteurs de forme du $D^{*}$ et du $D_{s}^{*}$. On utilise les mesures suivantes :

$-\tan ^{2}\left(\theta_{C}\right)=0,2250 \pm 0,0027[68]$

$-\mathcal{B}\left(B^{0} \rightarrow D^{*-} \pi^{+}\right)=(0,276 \pm 0,021) \%[68]$

- $\left.\mathcal{B}\left(B^{0} \rightarrow D_{s}^{*+} \pi^{-}\right)=1,9_{-1,3}^{+1,2} \pm 0,5\right) \times 10^{-5}[69,70]$

$-\frac{f_{D^{*}}}{f_{D_{s}^{*}}}=1,10 \pm 0,02[71]$

- $\mathcal{B}\left(D_{s}^{+} \rightarrow \phi \pi^{+}\right)=(4,71 \pm 0,47 \pm 0,35) \%[72]$.

Et on obtient $r^{*}=0,015_{-0,006}^{+0,004}$. L'équation 4.28 est obtenue en faisant deux approximations :

- L'amplitude $E$ du diagramme d'échange contribuant à la désintégration $B^{0} \rightarrow$ $D^{*+} \pi^{-}$a été négligée et on n'a considéré que l'amplitude $T$ du diagramme en arbre. En effet, on ne dispose pas d'une estimation fiable du terme d'échange pour ces désintégrations. On peut en revanche mesurer un rapport d'embranchement dû à un terme d'échange pour les désintégrations favorisées de Cabibbo: $\mathcal{B}\left(B^{0} \rightarrow D_{s}^{-} K^{+}\right)=$ $(3,5 \pm 0,9 \pm 1,0) 10^{-5}[73,74]$. On en déduit le rapport $\mathcal{B}\left(B^{0} \rightarrow D_{s}^{-} K^{+}\right) / \mathcal{B}\left(B^{0} \rightarrow\right.$ $\left.D_{-} \pi+\right)=10^{-2}$, ce qui confirme que les diagrammes d'échange sont fortement défavorisés par rapport aux diagrammes en arbre.

- On a également fait l'approximation que les effets de brisure de la symétrie $S U(3)$ étaient pris en compte au premier ordre par le rapport des constantes de désintégration $\frac{f_{D^{*}}}{f_{D_{s}^{*}}}$. On a estimé que les termes d'ordres supérieurs que l'on avait négligés étaient de l'ordre de quelques \% [75].

On assigne une erreur théorique relative plate, due à ces approximations, de $30 \%$. Cependant, les paramètres de violation de $C P$ que nous mesurons, comme par exemple $2 r^{*} \sin (2 \beta+\gamma) \cos (\delta)$, sont indépendants de toute incertitude théorique. C'est uniquement au niveau de l'interprétation des résultats que l'on peut être amené à utiliser cette estimation de $r^{*}$, comme on le verra au chapitre 6 . 


\subsubsection{Valeurs attendues pour les paramètres de $C P$}

Cette partie donne une estimation des valeurs attendues pour les paramètres de violation de $C P$ que l'on mesure dans l'analyse. Ces paramètres dépendent de $r^{*}, \beta, \gamma$ et $\delta$. La valeur de $r^{*}$ est évaluée dans la partie 4.2.3. La moyenne mondiale de $\sin (2 \beta)=$ $0,739 \pm 0,048$ [76]. La valeur pour $\gamma$, renvoyée par l'ajustement de CKMFitter [30], est $\gamma=62_{-12}^{+10}$ degrés. La factorisation QCD favorise une valeur de $\delta$ proche de 0 ou $\pi$. Avec ces valeurs, on s'attend à $\sin (2 \beta+\gamma) \sim 0,90, a=2 r^{*} \sin (2 \beta+\gamma) \cos (\delta) \sim \pm 0,027$ et $c=2 r^{*} \cos (2 \beta+\gamma) \sin (\delta) \sim 0$. Pour l'instant, on ne dispose pas d'estimation des paramètres $r^{\prime}$ et $\delta^{\prime}$. 


\section{5}

\section{Analyse}

\section{Introduction}

La désintégration $B^{0} \rightarrow D^{*-} \pi^{+}$permet l'étude de la violation de $C P$ dépendante du temps par la mesure du paramètre $\sin (2 \beta+\gamma)$. On s'attend à des asymétries très petites, de l'ordre de $2 \%$. On a donc besoin d'un maximum de statistique. Nous étudions la désintégration $B^{0} \rightarrow D^{*-} \pi^{+}$, suivie de la désintégration $D^{*-} \rightarrow D^{0} \pi^{-}$. Afin de sélectionner le maximum d'événements possible, on utilise une technique puissante de reconstruction partielle du $B$ qui se désintègre en $D^{*} \pi$ (que l'on appelle $B_{\text {rec }}$ ). Cela consiste à ne reconstruire que les deux pions issus des désintégrations du $B$ et du $D^{*}$, sans chercher à reconstruire le $D^{0}$. On calcule alors la masse de l'objet restant (voir la partie 5.1). Pour les événements de signal (correspondant à la désintégration $B^{0} \rightarrow D^{*-} \pi^{+}$), cette masse manquante, que l'on appelle $m_{\mathrm{rec}}$, pique à la masse du $D^{0}$. La méthode de reconstruction partielle permet d'augmenter la statistique d'un ordre de grandeur par rapport à une méthode de reconstruction exclusive, dans laquelle on reconstruit tous les produits de désintégration du $B$.

Toutefois, on a beaucoup plus de bruit de fond en utilisant la méthode de reconstruction partielle. Il convient donc de bien traiter les différents bruits de fond. On a en particulier beaucoup de bruit de fond de continuum, ce qui correspond à des événements $e^{+} e^{-} \rightarrow q \bar{q}$, où $q$ peut être un quark $u, d, s$ ou $c$. Il est possible de discriminer le continuum des événements $B \bar{B}$ (dans lesquels on a produit une paire $B \bar{B}$ ) en utilisant le fait que ces deux types d'événements ont des topologies très différentes. En effet, les événements $B \bar{B}$ ont une topologie plutôt sphérique, alors que les événements de continuum ont une topologie en deux jets. On combine alors plusieurs variables de forme d'événement dans un discriminant de Fisher $F$, lequel permet de discriminer ces deux types d'événements.

Pour résumer, on a donc trois distributions fondamentales à considérer dans cette analyse :

1. la distribution en masse manquante $m_{\mathrm{rec}}$, dans laquelle le signal pique à la masse du $D^{0}$,

2. la distribution de Fisher $F$ qui permet de discriminer les événements $B \bar{B}$ du continuum, 
3. la distribution en $\Delta t$ (où $\Delta t$ représente la différence de temps entre les désintégrations des deux $B$ ), car on étudie une asymétrie de $C P$ dépendante du temps.

D'autre part, on distingue deux catégories d'événements : les événements dont la saveur de $B_{\text {tag }}$ est étiquetée par un lepton et les événements dont la saveur de $B_{\text {tag }}$ est étiquetée par un kaon. Ces deux types d'événements ne sont comportent pas de la même façon, en particulier en ce qui concerne le vertex de $B_{\text {tag }}$, ce qui nous a amenés à faire deux analyses indépendantes pour chacune de ces deux catégories.

Notons que la méthode de reconstruction partielle a déjà été utilisée avec succès dans les expériences CLEO et ARGUS [77,78], ainsi que dans BABAR avec les désintégrations $B^{0} \rightarrow D^{*-} \pi^{+}$et $B^{0} \rightarrow D^{*-} \rho^{+}$pour mesurer le temps de vie des $B^{0}$ [79]. Cette analyse a conduit à la mesure $\tau_{B}^{0}=1.533 \pm 0.034$ (stat.) \pm 0.038 (syst.)ps et a permis de valider la procédure de reconstruction partielle.

Cependant, cette méthode apporte des difficultés supplémentaires dont il faut tenir compte dans l'analyse. En particulier, les traces provenant du $D^{0}$ non reconstruit peuvent être utilisées à tort pour étiqueter le deuxième $B$ de l'événement (que l'on appelle $B_{\text {tag }}$ ). On ne dispose donc d'aucune information sur la saveur de $B_{\text {tag }}$ pour ce genre d'événements. Ces traces, si elles sont utilisées pour déterminer le vertex de $B_{\text {tag }}$, biaisent la position de celui-ci et par conséquent $\Delta t$. On essaie donc de rejeter le maximum de traces provenant du $D^{0}$ en coupant sur un cône autour de la direction moyenne du $D^{0}$. Il subsiste cependant des traces du $D^{0}$ non reconstruit que l'on utilise à tort dans l'analyse. Il convient donc de prendre en compte cet effet.

\subsection{La méthode de reconstruction partielle}

\subsubsection{Principe de la méthode de reconstruction partielle}

Considérons la chaîne de désintégration $B \rightarrow D^{*} \pi_{f}$ suivie de $D^{*} \rightarrow D^{0} \pi_{s}$. Le pion issu de la première désintégration possède une grande impulsion et est donc indicé $f$ pour fast, alors que le pion issu de la désintégration du $D^{*}$ est beaucoup moins énergétique et est indicé ${ }_{s}$ pour slow. Le principe de la méthode de reconstruction partielle consiste à ne reconstruire que les deux pions, sans chercher à reconstruire la désintégration du $D^{0}$. Cette méthode permet d'augmenter la statistique d'un ordre de grandeur par rapport à une méthode de reconstruction exclusive du même mode de désintégration. Il est alors possible de calculer la masse manquante en partant du fait que les deux pions proviennent de la désintégration d'un $B$. La distribution en masse manquante du signal pique alors à la masse du $D^{0}$.

Cinq particules interviennent dans cette chaîne de désintégrations : le $B$, le $D^{*}$, le $\pi_{f}$, le $D^{0}$ et le $\pi_{s}$. On a donc besoin de 20 paramètres (4 pour chaque 4 -vecteur) pour décrire entièrement la cinématique. Cependant, on peut réduire ce nombre de paramètres à 6 en tenant compte de la conservation de l'énergie-impulsion des deux désintégrations, de la masse de chaque particule et du fait que l'on connaisse l'énergie de la collision $e^{+} / e^{-}$. Ainsi, si l'on mesure les trois composantes de l'impulsion de chacun des deux pions, la cinématique est entièrement déterminée. Le $D^{*}$ étant une particule de spin 1 , il est possible d'utiliser sa distribution d'hélicité pour discriminer le signal des bruits de fond. 
Toutefois, nous avons choisi une procédure légèrement différente dans laquelle on ne fait pas d'hypothèse sur la masse de la particule produite avec les deux pions dans la désintégration du $B$. Cela permet d'utiliser la distribution de la masse de la particule non reconstruite, $m_{\text {rec }}$, pour discriminer le signal des bruits de fond, le signal piquant à la masse du $D^{0}, m_{D}^{0}$. D'autre part, le fait d'imposer que la masse de la particule non reconstruite soit celle du $D^{0}$ n'a pas le même effet pour un événement tel que $m_{\text {rec }}$ est proche de $m_{D}^{0}$ ou pour un événement tel que $m_{\text {rec }}$ est très différente de $m_{D}^{0}$. C'est pourquoi nous avons décidé de ne pas faire d'hypothèse sur la masse de la particule non reconstruite et d'utiliser $m_{\text {rec }}$ comme une variable dans notre analyse.

Plaçons nous dans le référentiel du centre de masse de l' $\Upsilon(4 S)$. Comme la somme de la masse des deux $B$ produits par cette résonance est très proche de la masse de l' $\Upsilon(4 S)$, chaque $B$ est quasiment au repos dans ce référentiel. L'impulsion du $\pi_{f}$ est donc comprise dans un très petit intervalle autour de $2,25 \mathrm{GeV} / \mathrm{c}$. On commence par reconstruire le $\pi_{f}$, puis on l'associe à un autre pion de l'événement, de charge opposée, sans faire de coupure cinématique supplémentaire. On calcule ensuite la masse manquante dans $B_{\text {rec }}$ en considérant que les deux pions sont issus de la chaîne de désintégration mentionnée ici.

La conservation de l'énergie-impulsion pour chaque désintégration $\left(B^{0} \rightarrow D^{*-} \pi^{+}\right.$, suivie de $D^{*} \rightarrow X \pi$, où $X$ représente la particule non reconstruite) nous donne les relations suivantes sur les quadri-vecteurs :

$$
p_{B}=p_{\pi_{f}}+p_{D^{*}}, \quad p_{D^{*}}=p_{X}+p_{\pi_{s}}
$$

où $p_{i}$ représente le quadri-vecteur de la particule $i$. On peut en déduire la masse de la particule non reconstruite :

$$
m_{r e c}^{2}=\left(p_{B}-p_{\pi_{f}}-p_{\pi_{s}}\right)^{2}=m_{B}^{2}+\left(p_{\pi_{f}}+p_{\pi_{s}}\right)^{2}-2\left(E_{B}\left(E_{\pi_{f}}+E_{\pi_{s}}\right)-\overrightarrow{p_{B}} \cdot\left(\overrightarrow{p_{\pi_{f}}}+\overrightarrow{p_{\pi_{s}}}\right)\right) .
$$

Cette quantité est particulièrement bien adaptée pour sélectionner le signal. Comme cela a été dit précédemment, on ne mesure que les impulsions des deux pions. Il faut donc calculer le 4 -vecteur du $B$. Pour cela, on se place dans un repère orthonormé $(\vec{u}, \vec{v}, \vec{z})$ tel que l'axe $\vec{z}$ soit parallèle à la direction du $\pi_{f}$ et que l'axe $\vec{u}$ soit dans le plan défini par les directions des pions $\pi_{f}$ et $\pi_{s}$. Ce repère est représenté figure 5.1. On peut voir sur la figure les directions des particules $B, \pi_{f}$ et $\pi_{s}$ données par leurs vecteurs impulsion. On appelle $\theta_{B}$ l'angle entre les directions du $B$ et du $\pi_{f}$ et $\phi$ l'angle azimuthal. On note également $\alpha$ l'angle entre les directions de $\pi_{f}$ et de $\pi_{s}$.

Dans ce repère, on a :

$$
\overrightarrow{p_{B}}=\left|\overrightarrow{p_{B}}\right|\left(\cos \left(\theta_{B}\right) \vec{z}+\sin \left(\theta_{B}\right) \cos (\phi) \vec{u}+\sin \left(\theta_{B}\right) \sin (\phi) \vec{v}\right) .
$$

On peut déterminer la valeur de $\cos \left(\theta_{B}\right)$ en élevant la relation $p_{B}=p_{\pi_{f}}+p_{D^{*}}$ au carré. On obtient :

$$
\cos \left(\theta_{B}\right)=\frac{m_{B}^{2}+m_{\pi}^{2}-m_{D^{*}}^{2}-2 E_{B} E_{\pi_{f}}}{2\left|\overrightarrow{p_{B}}\right|\left|\overrightarrow{p_{\pi_{f}}}\right|} .
$$

D'autre part, si on néglige la largeur de la résonance $\Upsilon(4 S)$, on a $E_{B}=\sqrt{s} / 2$, où $\sqrt{s}$ représente l'énergie de la collision dans le centre de masse des faisceaux. On peut ainsi 


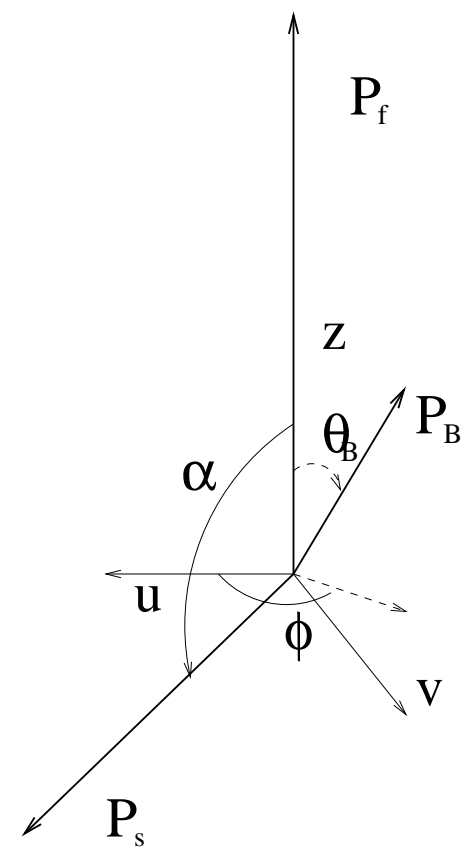

FiG. 5.1: Définition du repère $(\vec{u}, \vec{v}, \vec{z})$

exprimer la masse manquante $m_{\text {rec }}$ sans avoir à reconstruire exclusivement le méson $B$, mais uniquement en connaissant les 4 -vecteurs des deux pions. Notons cependant que l'on ne connaît pas l'angle azimuthal $\phi$. La quantité que l'on considère n'est donc pas exactement la masse manquante mais plutôt une pseudo-masse manquante, qui est la moyenne entre la valeur maximale et la valeur minimale de $m_{\text {rec }}$ que l'on peut obtenir, sachant que la valeur maximale de $m_{\text {rec }}$ correspond à $\cos (\phi)=1$ et sa valeur minimale à $\cos (\phi)=-1$. L'angle $\phi$ apparaît uniquement dans le terme de l'équation 5.2 suivant :

$$
\begin{aligned}
& \overrightarrow{p_{B}} \cdot\left(\overrightarrow{p_{\pi_{f}}}+\overrightarrow{p_{\pi_{s}}}\right)= \\
& \left|\overrightarrow{p_{B}}\right|\left|\overrightarrow{p_{\pi_{f}}}\right| \cos \left(\theta_{B}\right)+\left|\overrightarrow{p_{B}}\right|\left|\overrightarrow{p_{\pi_{s}}}\right| \cos \left(\theta_{B}\right) \cos (\alpha)+\left|\overrightarrow{p_{B}}\right|\left|\overrightarrow{p_{\pi_{s}}}\right| \sin \left(\theta_{B}\right) \sin (\alpha) \cos (\phi) .
\end{aligned}
$$

La cinématique particulière de la chaîne de désintégration fait que le $B$ est quasiment au repos dans le référentiel du centre de masse de l' $(4 S)\left(p_{B} \sim 300 \mathrm{MeV} / c\right)$ et que le $\pi_{s}$ est quasiment au repos dans le référentiel du centre de masse du $D^{*}\left(p_{\pi_{s}}<250 \mathrm{MeV} / c\right)$. Les impulsions $p_{B}$ et $p_{\pi_{s}}$ sont donc petites et l'erreur induite par cette approximation est faible car le terme dans lesquel $\phi$ apparait est petit par rapport aux autres termes de l'équation 5.2. Cependant, cette erreur est importante par rapport aux erreurs sur la reconstruction des traces des pions. La résolution moyenne sur la masse manquante est de l'ordre de $3 \mathrm{MeV} / c^{2}$, comme le montre la figure 5.2, qui représente la distribution de masse manquante pour des événements de signal Monte-Carlo.

Pour l'analyse, on a besoin de définir une direction de référence pour la particule non reconstruite. On détermine cette direction en choisissant $\phi=0$. 


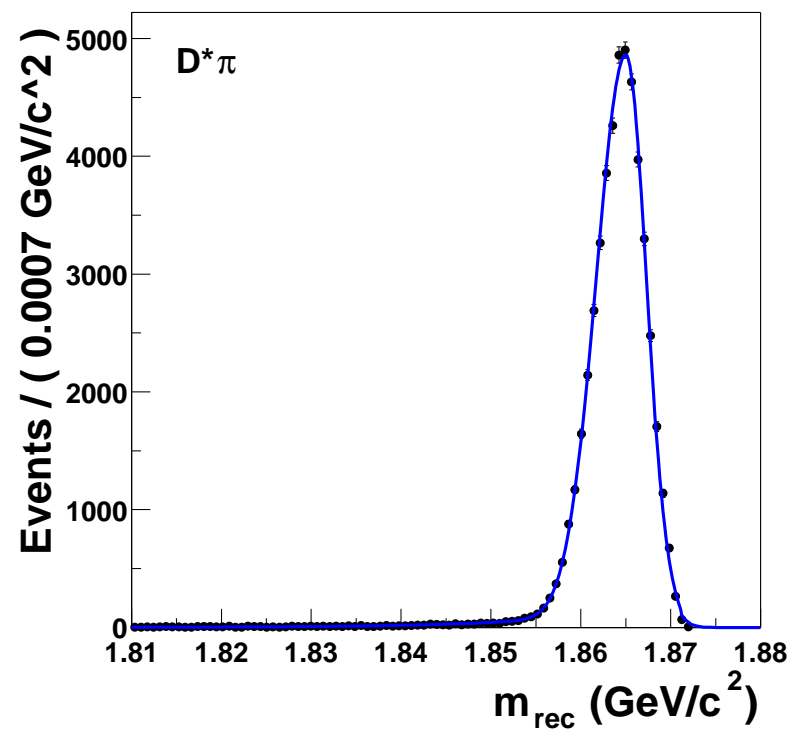

FIG. 5.2: Distribution de masse manquante pour des événements simulés de signal

\subsubsection{La masse manquante $: m_{\text {rec }}$}

Une variable très importante de l'analyse et qui est propre à la méthode de reconstruction partielle est la masse manquante $m_{\mathrm{rec}}$. Elle correspond à la masse de l'objet non-reconstruit et son expression est donnée par l'équation 5.2. Cette variable est particulièrement utile pour discriminer le signal du bruit de fond $B \bar{B}$. On distingue quatre grandes catégories de bruit de fond :

- le continuum;

- les désintégrations $B^{0} \rightarrow D^{*-} \rho^{+}$;

- le bruit de fond qui pique en masse manquante : ces événements sont composés d'une paire de traces provenant du même méson $B$ et dont l'une d'elles est un pion provenant de la désintégration d'un $D^{*}\left(\mathrm{ex}: B \rightarrow D^{* *+} \pi^{-}\right)$;

- le bruit de fond combinatoire $B \bar{B}$, dont la distribution est plate en masse manquante et qui regroupe toutes les autres catégories de bruit de fond possibles.

Il est crucial de bien séparer le bruit de fond du signal dans cette analyse, car il y a beaucoup de bruit de fond. En effet, parmi les événements étiquetés par un lepton (kaon), $12 \%$ (50\%) sont des événements de continuum et $43 \%(27 \%)$ du bruit de fond $B \bar{B}$.

Les différents types d'événements ont des distributions différentes en masse manquante : le signal $D^{*} \pi$ pique bien évidemment à la masse du $D^{0}\left(m_{D}^{0}=1864,6 \pm 0,5 \mathrm{MeV}\right.$ [29]), mais le bruit de fond $D^{*} \rho$, ainsi que le bruit de fond qui pique, également. En revanche, le combinatoire et le continuum ont une distribution beaucoup plus plate.

La distribution en masse manquante des différents types d'événements $B \bar{B}$ est représentée figure 5.3. On peut distinguer deux parties :

- la région du signal : 1,845< $m_{\mathrm{rec}}<1,880 \mathrm{GeV} / c^{2}$, dans laquelle se trouve la majorité du signal, ainsi que du bruit de fond. 
- la région latérale : $1,810<m_{\text {rec }}<1,840 \mathrm{GeV} / c^{2}$, qui ne contient que du bruit de fond. Cette région est utilisée pour paramétrer le bruit de fond.
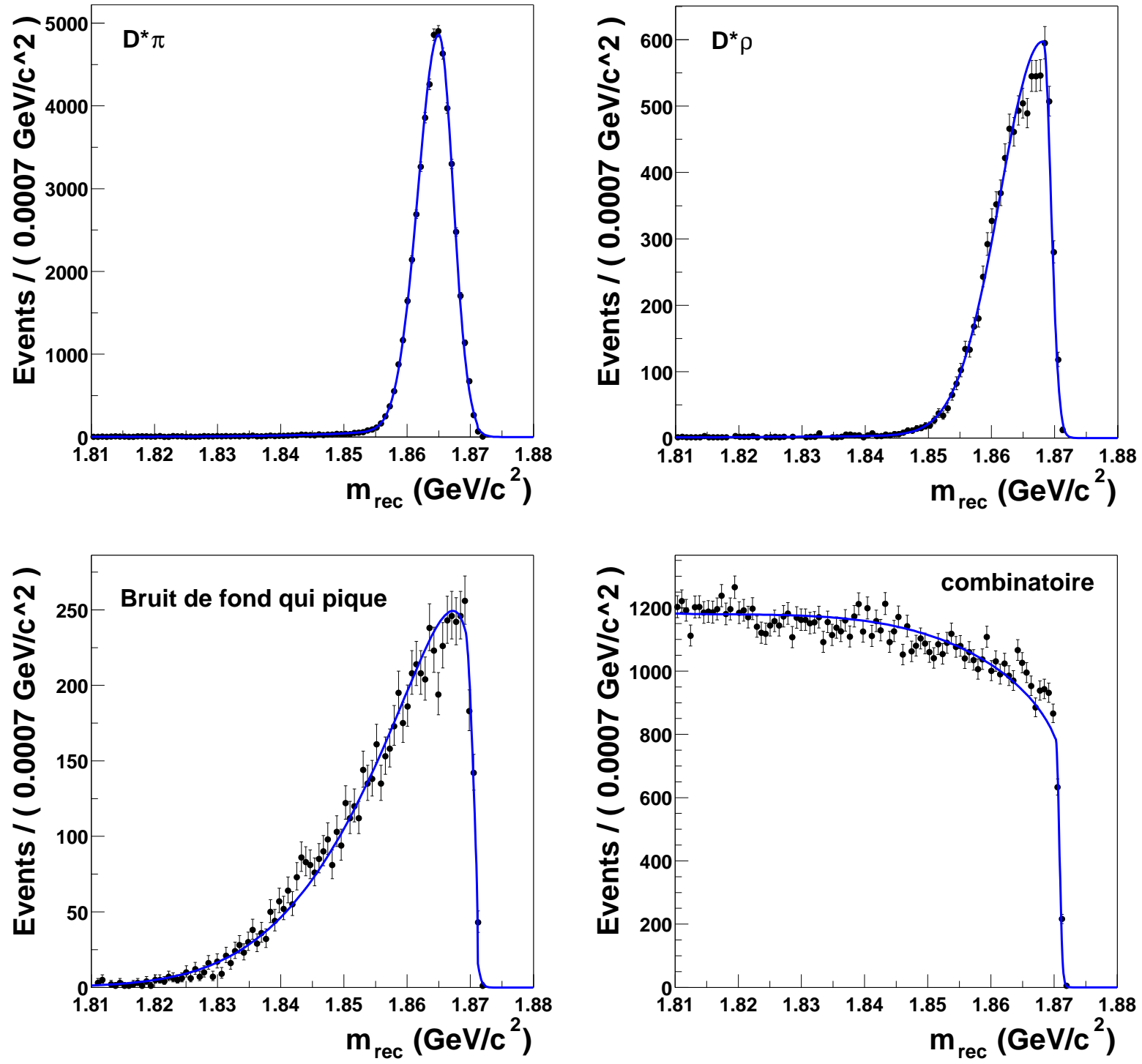

Fig. 5.3: Distribution de $m_{\text {rec }}$ pour les différents types d'événements $B \bar{B}$ de Monte-Carlo. Les quatre distributions représentent, de gauche à droite et de haut en bas, les distributions en $m_{\mathrm{rec}}$ des événements $D^{*} \pi, D^{*} \rho$, du bruit de fond qui pique et du bruit de fond combinatoire.

\subsubsection{Le discriminant de Fisher $F$}

Le discriminant de Fisher est une variable qui permet principalement de discriminer les événements $B \bar{B}$ du continuum. Cela est particulièrement important pour l'analyse des 
événements étiquetés par un kaon, car parmi les événements sélectionner, 50 \% sont des événements de continuum.

Les mésons $B$ sont produits quasiment au repos dans le référentiel du centre de masse des faisceaux : leur désintégration est donc pratiquement isotrope. En revanche, les événements de continuum $q \bar{q}$ ont une structure en deux jets énergétiques très prononcée, en particulier lorsqu'il s'agit de quarks légers $(u, d, s)$. Cette différence de configuration permet de discriminer ces deux types d'événements.

Le discriminant utilisé combine 15 variables de forme d'événement avec la méthode de Fisher [80,81]. Soit $\left\{X_{i}\right\}_{i=1 \ldots 15}$ l'ensemble des variables que l'on mesure pour un événement donné. Le discriminant de Fisher est une combinaison linéaire de ces variables :

$$
F=\sum_{i=1}^{15} \alpha_{i} X_{i}, \quad \alpha_{i}=\sum_{j=1}^{15}\left(\mu_{i}^{s i g}-\mu_{i}^{\text {cont }}\right)_{j}\left(\operatorname{cov}^{\text {sig }}+\operatorname{cov}^{\text {cont }}\right)_{j i}^{-1}
$$

Les coefficients $\alpha_{i}$ sont déterminés avec du Monte-Carlo de la façon suivante:

- on considère deux populations d'événements : signal et continuum et on calcule les $\left\{X_{i}\right\}$ pour chaque événement,

- on calcule le barycentre pour chaque population dans l'espace à 15 dimensions : on obtient $\left\{\mu_{i}^{\text {sig }}\right\}$ et $\left\{\mu_{i}^{\text {cont }}\right\}$,

- les termes $\operatorname{cov}_{i j}$ sont les éléments de la matrice de covariance de chaque population.

Les 15 variables utilisent deux lots de particules par événement :

- le lot 1 qui comporte toutes les traces et tous les dépôts dans le calorimètre à part ceux attribués aux deux pions,

- le lot 2 qui contient les particules du lot 1, sauf celles comprises dans un cône d'angle d'ouverture de 1,25 rad autour de la direction de référence du $D^{0}$.

L'idée est de sélectionner les particules produites par le $B_{\text {tag }}$. On utilise ces deux lots de particules pour construire les 15 variables discriminantes suivantes :

- la somme scalaire des impulsions des traces et dépôts dans le référentiel du centre de masse pour 9 intervalles de $20^{\circ}$ autour de la direction du pion dur avec le lot 1 ;

- la valeur de la sphéricité [20] calculée avec le lot 1. La sphéricité est la somme normalisée du carré des impulsions transverses de chaque trace par rapport à l'axe de l'événement. Sa valeur est comprise entre 0 et 1 et est proche de 1 pour des événements isotropes ;

- l'angle entre le pion dur et l'axe de sphéricité calculé avec les particules du lot 2;

- l'impulsion de toutes les particules du lot 2 ;

- l'impulsion du pion dur et son angle polaire calculés dans le référentiel du centre de masse.

Chacune de ces variables n'est pas très discriminante en tant que telle, mais la combinaison de toutes ces variables dans un discriminant de Fisher permet de différencier efficacement le $B \bar{B}$ du continuum. La distribution de $F$ pour les événements étiquetés par un kaon est représentée figure 5.4. 


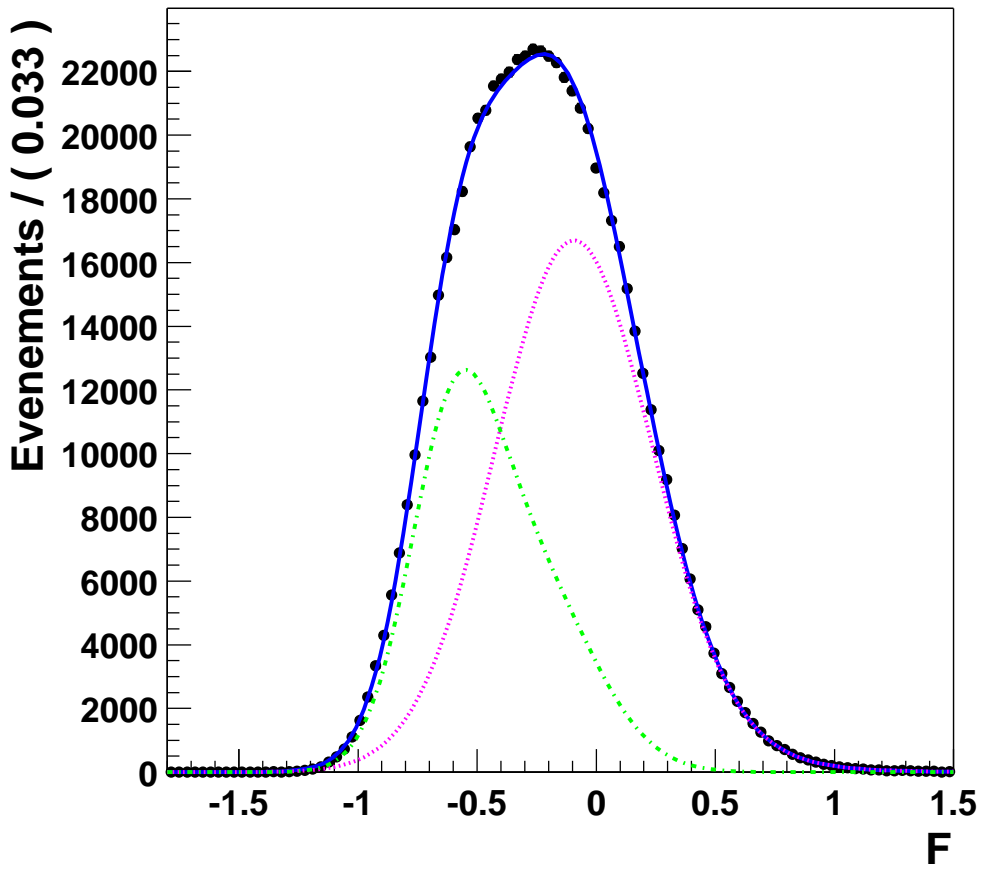

FIG. 5.4: Distribution de $F$ pour les événements étiquetés par des kaons, enregistrés sous la résonance. La courbe centrée autour de 0 représente la contribution du continuum et la courbe centrée autour de $-0,6$ celle du $B \bar{B}$. 

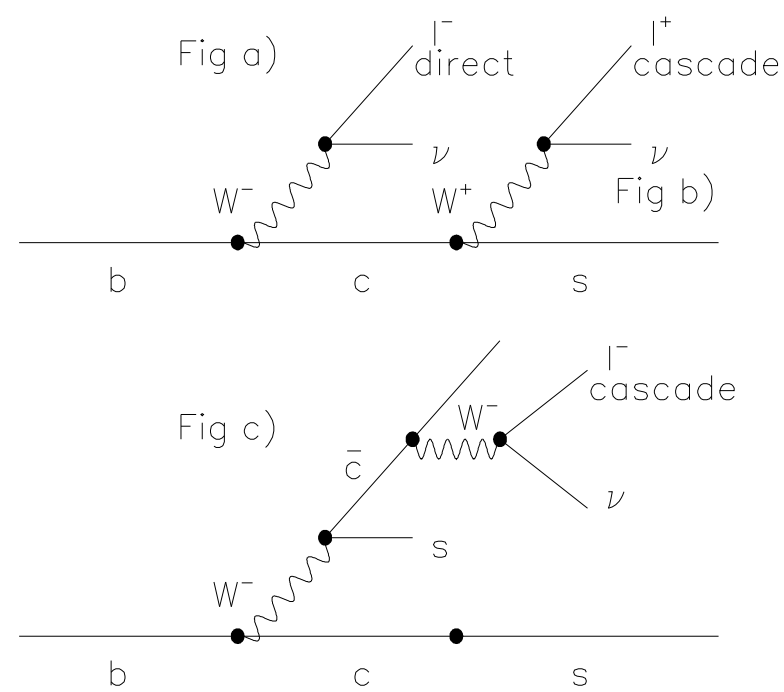

FIG. 5.5: Production de lepton direct (a), de cascade avec une charge de signe opposé à la charge du $b$ (b) et de cascade avec une charge de signe identique à la charge du $b$ (c).

\subsection{4 Étiquetage de la saveur du $B$}

Ainsi que cela a été expliqué dans la partie 1.2.3, on étiquette la saveur de $B_{\text {tag }}$ par la reconstruction inclusive de ses produits de désintégration. Il existe en effet des corrélations entre la saveur du quark $b$ et le signe des charges des produits de désintégrations du $B$. La plupart des événements sont étiquetés par un lepton ( $e$ ou $\mu$ ) ou par un kaon.

Considérons le cas où $B_{\text {tag }}$ est étiqueté par un lepton. Si le lepton est directement produit par la désintégration semi-leptonique du méson $B$, alors la charge du quark b est de même signe que la charge du lepton. On les appelle leptons directs. Par opposition, les leptons non directs sont appelés leptons de cascade. Leur charge peut alors être de signe opposé ou identique à la charge du quark $b$. La figure 5.5 représente les différentes corrélations possibles entre la saveur du $B$ et la charge du lepton étiquetant.

Il n'est pas possible de distinguer dans l'analyse le type de lepton étiquetant. On peut cependant réduire le nombre de leptons de cascade en coupant à $1,1 \mathrm{GeV} / \mathrm{c}$ sur l'impulsion du lepton dans le référentiel du centre de masse. En effet, les leptons directs ont une impulsion plus grande que les leptons de cascade. Dans l'analyse, environ $12 \%$ des événements étiquetés par un lepton le sont par un lepton de cascade.

Les kaons utilisés pour étiqueter la saveur de $B_{\text {tag }}$ sont majoritairement produits par la cascade $b \rightarrow c \rightarrow s$ et leur charge est principalement de signe opposé à la charge du $b$. La fraction de mauvais étiquetage pour les événements étiquetés par un kaon $(\sim 16 \%)$ est bien plus importante que pour les événements étiquetés par un lepton $(\sim 7 \%)$, mais il y a beaucoup plus d'événements étiquetés par un kaon (environ trois fois plus dans cette analyse).

La méthode de reconstruction partielle nous a conduit à utiliser un algorithme d'éti- 
quetage standard, mais légèrement modifié de BABAR. En effet, le $D^{0}$ non reconstruit introduit des traces supplémentaires qui pourraient être utilisées à tort pour étiqueter l'événement. L'information n'est alors pas exploitable car la trace provient de $B_{\text {rec }}$ et non de $B_{\text {tag. }}$. Cet effet est pris en compte dans la paramétrisation des fonctions probabilité de densité (PDF), comme on le verra dans la suite. Afin de ne pas compliquer inutilement l'analyse, il est préférable de n'utiliser qu'une seule trace pour l'étiquetage. Un des algorithmes standard de BABAR, appelé Elba tagger [82], est particulièrement adapté à ce cas de figure.

Cet algorithme exploite diverses méthodes. Dans le cas idéal où $B_{\text {tag }}$ se désintègre en un lepton ou un kaon clairement identifié, de simples coupures suffisent. Environ $45 \%$ des événements rentrent dans ce cas de figure. Dans cette analyse, nous n'utilisons que ces événements que nous séparons en deux catégories selon que la particule qui étiquette est un lepton ou un kaon : cela conduit à deux analyses séparées.

Si plusieurs traces étiquetantes sont présentes dans l'événement, on choisit celle dont l'angle $\theta_{t a g}$ avec la direction de référence du $D^{0}$ non-reconstruit, dans le référentiel du centre de masse, est le plus grand. Cela permet de minimiser le risque d'utilisation d'une trace de l'objet non reconstruit pour l'étiquetage. On ne conserve que les traces telles que $\cos \left(\theta_{\text {tag }}\right)<0,75$ pour les leptons et telles que $\cos \left(\theta_{\text {tag }}\right)<0,50$ pour les kaons, afin de limiter le nombre d'événements étiquetés par une trace du $D^{0}$ non reconstruit. Ces coupures ont été déterminées à l'aide des distributions de $\cos \left(\theta_{\text {tag }}\right)$ montrées figures 5.6 et 5.7. L'efficacité de cette coupure est de $94 \%$ (86\%) pour des événements de signal étiquetés par un lepton (kaon) provenant de $B_{\text {tag }}$ et est de $5 \%(21 \%)$ pour des événements de signal étiquetés par un lepton (kaon) provenant du $D^{0}$ non reconstruit. Cette coupure permet donc de sélectionner la plupart du signal étiqueté par le $B_{\text {tag }}$ et d'éliminer une grande partie du signal étiqueté par une trace du $D^{0}$. Elle est particulièrement performante pour les événements étiquetés par un lepton.

Si un événement peut être étiqueté à la fois par un lepton et par un kaon, on n'utilise que l'information du lepton, qui est beaucoup plus propre.

Notons que l'algorithme distingue deux autres catégories d'événements qui ne sont pas étiquetés de façon aussi claire. Dans ce cas-là, un réseau de neurones est utilisé et la valeur renvoyée informe sur la saveur de $B_{\mathrm{tag}}$. On utilise plusieurs traces pour étiqueter la saveur du $B_{\text {tag }}$ de ce type d'événements. Cette technique n'est pas facilement exploitable avec une méthode de reconstruction partielle, dans laquelle on évite au maximum d'utiliser à tort les traces issues du $D^{0}$ non reconstruit. Utiliser ces catégories reviendrait donc à compliquer énormément l'analyse, alors que l'amélioration apportée par ces catégories supplémentaires est très faible. On considère donc que ces événements ne sont pas utilisables pour une analyse utilisant une méthode de reconstruction partielle.

\subsubsection{Mesure de la distance entre les vertex des deux $B$}

L'analyse de la violation de $C P$ dans la désintégration $B^{0} \rightarrow D^{*-} \pi^{+}$est dépendante du temps. Il est nécessaire de connaître la différence de temps $\Delta t$ entre les désintégrations des deux $B$. Pour cela, on doit reconstruire les deux vertex. 


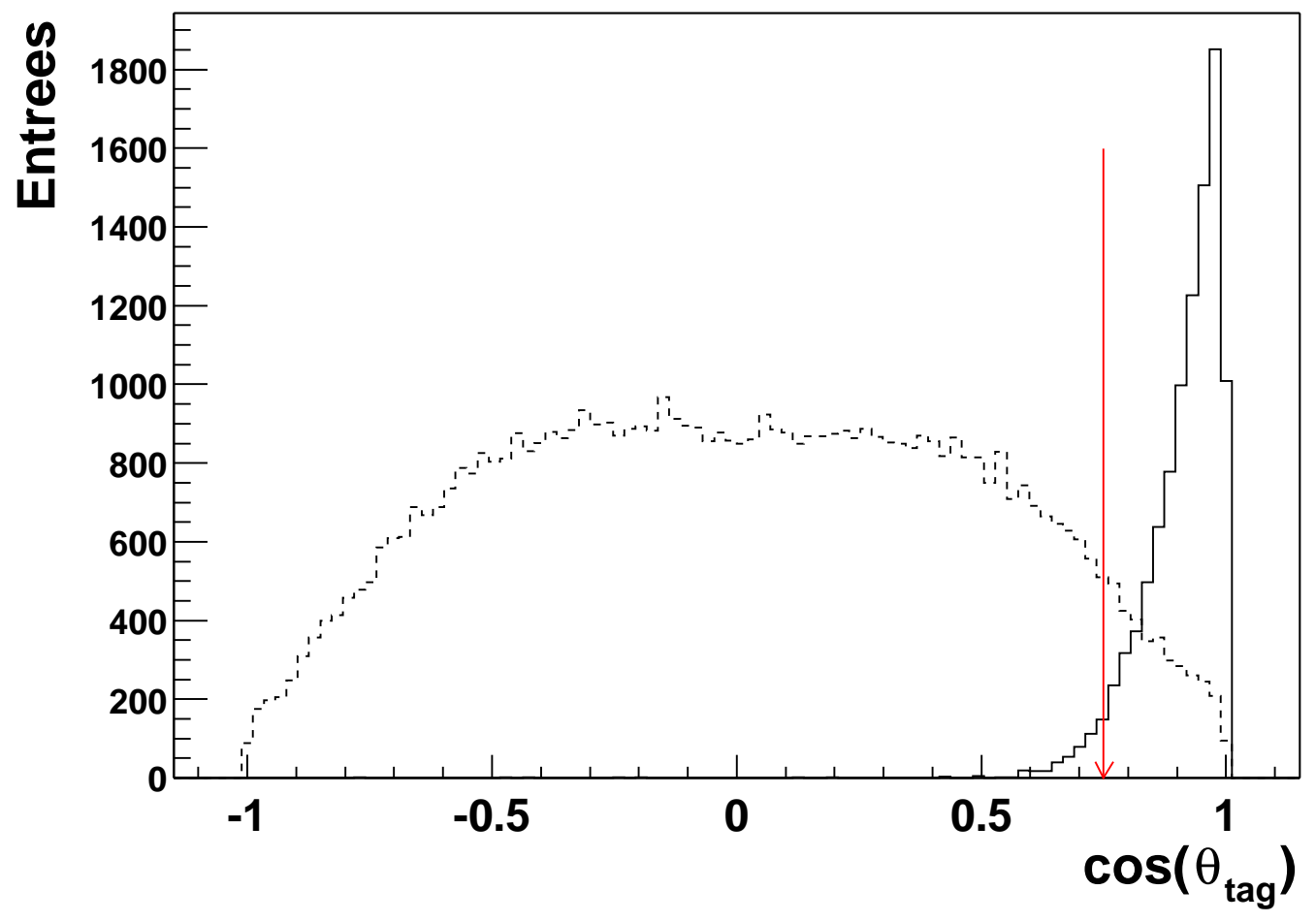

FIG. 5.6: Distribution de $\cos \theta_{\text {tag }}$ pour des événements simulés de signal, étiquetés par un lepton. La ligne pleine (en pointillés) correspond aux traces étiquetantes provenant du $D^{0}$ $\left(B_{\mathrm{tag}}\right)$. La flèche indique la coupure appliquée $\left(\cos \theta_{\operatorname{tag}}<0,75\right)$.

\section{Ajustement du vertex de $B_{\text {rec }}$}

On cherche à déterminer la position de la désintégration de $B_{\text {rec }}$ en $D^{*} \pi$. Pour cela, on a d'abord besoin de connaître la position du point d'interaction des faisceaux, qui correspond au point de production de la résonance $\Upsilon(4 S)$. Cette position se trouve dans un ellipsoïde dont la dimension longitudinale est de l'ordre de $8 \mathrm{~mm}$ et la position transverse est de l'ordre de $5 \mu \mathrm{m}$ selon l'axe $y$ et de $200 \mu \mathrm{m}$ selon l'axe $x$. Pour déterminer la position du centre de l'ellipsoïde, on utilise des événements de type di-muon ou Bhabhas $\left(e^{+} e^{-} \rightarrow\right.$ $\left.l^{+} l^{-}\right)$qui ont deux traces dures chargées dans l'état final. On peut alors déterminer la distance de plus proche approche à l'origine de ces deux traces dans le plan transverse, ce qui nous donne le point d'interaction des faisceaux dans le plan transverse et on en déduit la position longitudinale par de simple considérations géométriques. Les paramètres de la position du point d'interaction sont mesurés à chaque run.

Pour les événements $B \bar{B}$, il y a production de la résonance $\Upsilon(4 S)$ au point d'interaction. Il faut alors tenir compte du vol du $B$ afin de déterminer la position de sa désintégration. Le facteur de Lorentz important de la machine (voir section 2.1) implique que les $B$ volent principalement dans la direction longitudinale. Cependant, ils volent également dans la direction transverse. Plaçons nous dans le référentiel de l' $(4 S)$. Par conservation de l'énergie-impulsion, l'énergie du $B$ est $E_{B}=M_{\Upsilon(4 S)} / 2=5290 \mathrm{MeV}$ et 


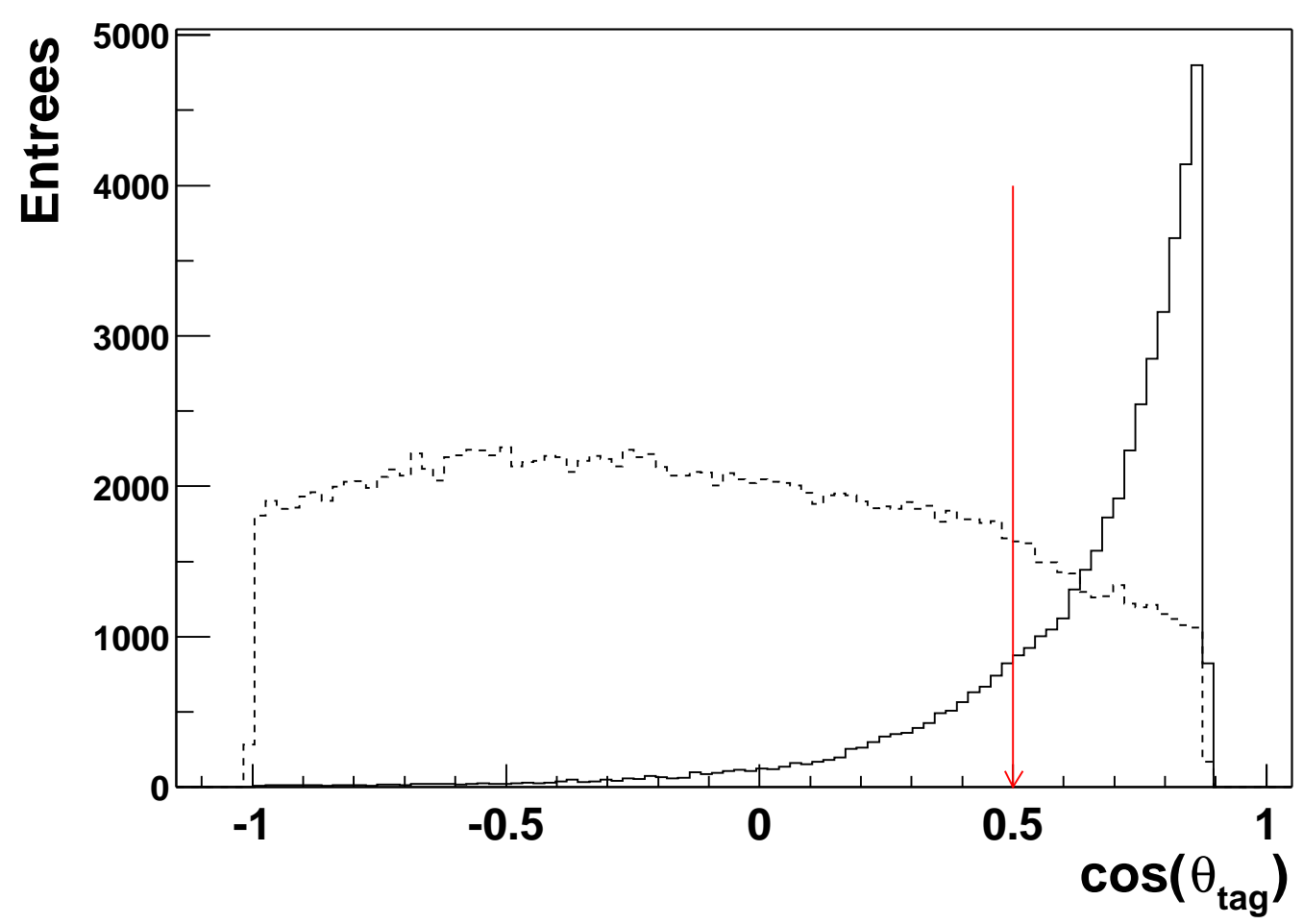

Fig. 5.7: Distribution de $\cos \theta_{\text {tag }}$ pour des événements simulés de signal, étiquetés par un kaon. La ligne pleine (en pointillés) correspond aux traces étiquetantes provenant du $D^{0}$ $\left(B_{\text {tag }}\right)$. La flèche indique la coupure appliquée $\left(\cos \theta_{\operatorname{tag}}<0,50\right)$.

son impulsion est $p_{B}=\sqrt{E_{B}^{2}-M_{B}^{2}} \sim 335 \mathrm{MeV}$. On a donc $\gamma=E_{B} / M_{B}=1,002$ et $\beta=p_{B} / M_{B}=0,063$. Le $B$ vole pendant un temps caractéristique égale à sa durée de vie $\tau_{B} \sim 1,536 \mathrm{ps}$. Le distance parcourue par le $B$ dans le référentiel de l' $\Upsilon(4 S)$ est donc de l'ordre de $d=\beta \gamma c \tau_{B}=29 \mu \mathrm{m}$. On considère que l'ordre de grandeur du vol des $B$ dans le plan transverse est de $25 \mu \mathrm{m}$. C'est pourquoi on élargit la taille de l'ellipsoïde dans le plan transverse. On peut alors supposer légitimement que $B_{\text {rec }}$ se trouve dans cet ellipsoïde au moment de sa désintégration.

Afin de déterminer la position de la désintégration de $B_{\text {rec}}$, on utilise la direction de ses produits de désintégration. Dans le cas de la méthode de reconstruction partielle, on ne peut utiliser que les traces des deux pions. Cependant, le pion mou issu de la désintégration du $D^{*}$ a une impulsion très faible et subit de nombreuses diffusions dans la paroi du tube à vide et dans la matière constituant le détecteur. La résolution sur la position de la trace de ce pion est trop mauvaise pour apporter de l'information sur la position du vertex. On utilise donc uniquement la direction du pion dur, issu directement du $B$.

Pour reconstruire le vertex de $B_{\text {rec}}$, on ajuste la trace du $\pi_{f}$ avec la contrainte, décrite précédemment, sur la position du faisceau. Ensuite on déduit la position longitudinale $z_{\text {rec }}$ grâce à la direction du pion. On demande que la probabilité de l'ajustement du vertex de $B_{\text {rec }}$ soit supérieure à 0,001 . 


\section{Ajustement du vertex de $B_{\text {tag }}$}

Pour reconstruire le vertex du $B_{\text {tag }}$ d'un événement étiqueté par un kaon, on ajuste toutes les traces de l'événement, autres que les traces des deux pions, avec une contrainte sur la position du faisceau. Afin de ne pas attribuer au $B_{\text {tag }}$ des traces venant du $D^{0}$ non reconstruit qui biaiseraient la position du vertex, on n'utilise pas les traces comprises dans un cône d'angle d'ouverture égal à 1 rad autour de la position de référence de la particule non reconstruite. Si la contribution d'une trace au $\chi^{2}$ est supérieure à 6 , on exclut cette trace et on réajuste le vertex, et ce jusqu'à ce qu'aucune trace n'ait une telle contribution. Cela permet d'éviter d'avoir une trace trop mauvaise, qui risquerait de biaiser le vertex.

Pour les événements étiquetés par un lepton, on utilise a même procédure que celle utilisée pour ajuster le vertex de $B_{\text {rec }}$, mais on remplace le pion dur par le lepton. Cette procédure permet de déterminer la position du vertex non biaisée par les traces du $D^{0}$ non reconstruit.

L'algorithme de reconstruction du vertex du $B_{\text {tag }}$, renvoie sa position, qui est appelée $z_{\text {tag }}$.

\section{Calcul de $\Delta t$}

La variable $\Delta t$ est obtenue à partir des mesures des positions longitudinales des deux vertex $z_{\text {rec }}$ et $z_{\text {tag. }}$. On calcule alors $\Delta z=z_{\mathrm{rec}^{-}} z_{\text {tag }}$. Les deux $B$ sont produits avec une très grande vitesse le long de l'axe $z$ dans le référentiel du laboratoire, avec un facteur de Lorentz $\beta \gamma=0,56$. Ils peuvent cependant avoir une faible impulsion transverse. On appelle $d$ la distance entre les deux vertex et $\epsilon$ la distance dans le plan transverse (voir figure 5.8).

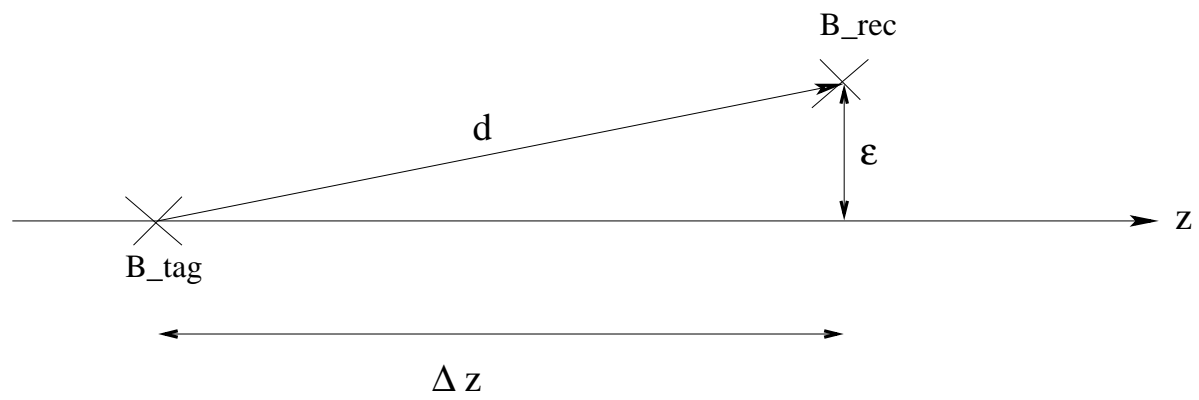

Fig. 5.8: Schéma des vertex des deux $B$

$\epsilon$ est de l'ordre de $25 \mu \mathrm{m}$ et $\Delta z$ est de l'ordre de $260 \mu \mathrm{m}$. On peut donc négliger la composante transverse avec une très bonne approximation et calculer $\Delta t$ en utilisant la formule $\Delta t=\Delta z / \beta \gamma c$. Les paramètres $\beta$ et $\gamma$ sont déterminés run par run (un run correspond à environ 45 minutes de prises de données) en utilisant les énergies mesurées des faisceaux. On néglige donc la variation de ces paramètres événement par événement, dont l'impact est très faible sur l'erreur sur $\Delta t$ (notée $\sigma_{\Delta t}$ ). Cette erreur provient de l'incertitude sur les contraintes utilisées dans l'ajustement des deux vertex (positions des traces et du point d'interaction). 
Des études sur des événements simulés de signal ont conduit à couper sur l'erreur de $\Delta t: \sigma_{\Delta t}<2 \mathrm{ps}$ (voir figure 5.9). On applique également la coupure suivante : $|\Delta t|<15 \mathrm{ps}$, afin de supprimer les événements aberrants.

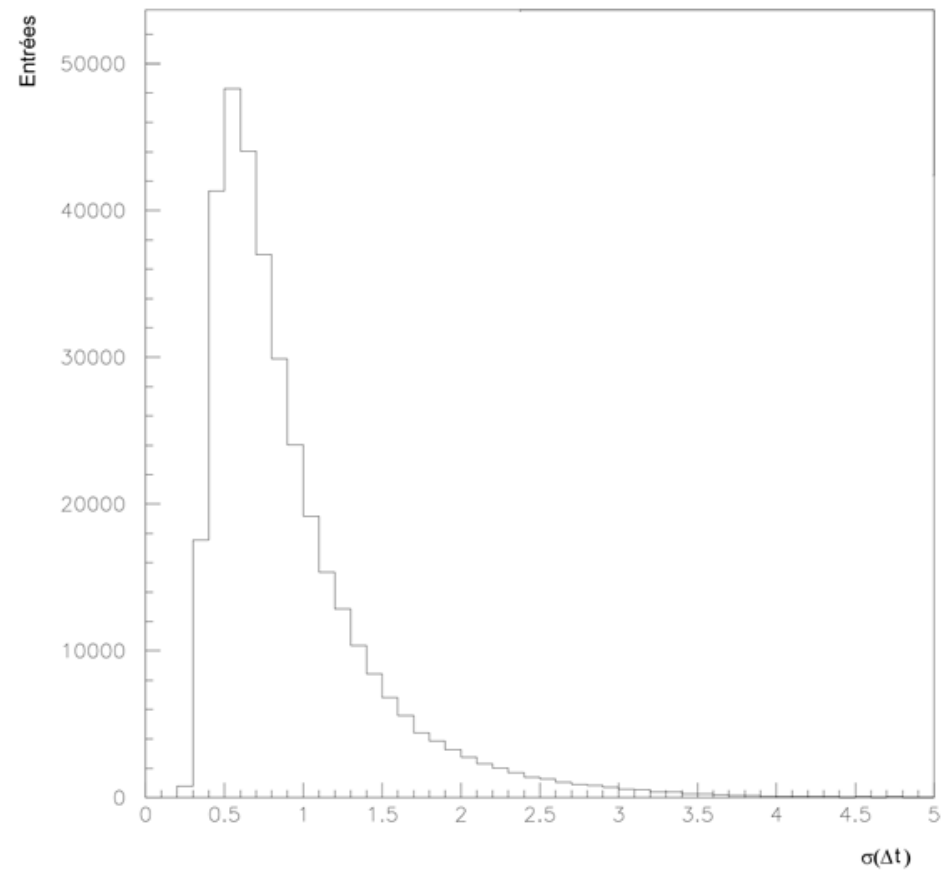

Fig. 5.9: Distribution de l'erreur sur $\Delta t$ (en $p s$ ) pour un échantillon d'événements simulés de signal

\section{2 Événements utilisés}

Dans le cadre de la méthode de reconstruction partielle, on cherche à sélectionner des événements avec deux pions chargés de charges opposées provenant de la désintégration d'un $B^{0}$ et avec une masse manquante dans l'événement correspondant à un $D^{0}$.

\subsubsection{Présélection au niveau de la production}

Dans BABAR les événements ayant passé le système de déclenchement sont enregistrés et forment un échantillon de données appelé AllEvents qui sert alors à la production de données sous un format exploitable pour l'analyse. Afin de ne pas avoir à reconstruire tous les événements pour ensuite les sélectionner, on fait d'abord une présélection rapide au niveau de la production afin de ne reconstruire qu'un sous-échantillon, appelé skim. À ce niveau-là, on sélectionne les événements ayant au moins deux traces chargées. 
On requiert qu'au moins une des deux traces ait une impulsion dans le centre de masse comprise entre 2,05 et 2,45 GeV/c. Cela permet de sélectionner une trace pouvant être le pion de haute impulsion. Cette coupure correspond à une valeur de $\left|\cos \left(\theta_{B}\right)\right|<1,3$. On permet à $\left|\cos \left(\theta_{B}\right)\right|$ d'être plus grand que 1 afin de tenir compte des effets de détecteur. Le pion dur doit satisfaire les conditions d'une liste appelée GoodTracksLoose :

1. la trace doit avoir au moins 12 points de mesure dans la chambre à fils,

2. le point de plus proche approche de la trace par rapport au point d'interaction des faisceaux doit être à moins de $1,5 \mathrm{~cm}$ dans le plan transverse et à moins de $10 \mathrm{~cm}$ selon l'axe longitudinal $z$,

3. l'impulsion transverse doit être au minimum de $0,1 \mathrm{GeV} / \mathrm{c}$.

On impose également des coupures supplémentaires relatives à l'identification des particules afin de ne pas sélectionner d'électron, de muon ou de kaon.

On sélectionne alors la trace du pion mou, provenant de la désintégration du $D^{*}$, qui doit satisfaire aux conditions dites GoodTracksVeryLoose. Ces conditions sont les mêmes que celles du pion dur, mais on ne demande pas de points de mesure dans la chambre à fils et il n'y a pas d'impulsion transverse minimum.

On fait de plus une coupure sur le rapport $R 2$ qui est le moment de Fox-Wolfram d'ordre 2, normalisé par le moment de Fox-Wolfram d'ordre 0. Le moment de Fox-Wolfram [83] d'ordre $l H_{l}$ est une observable qui caractérise la forme d'un état final provenant de l'annihilation $e^{+} e^{-}$et qui est indépendante du système de coordonnées utilisé. $H_{l}$ dépend de l'impulsion des particules produites ainsi que des angles entre ces particules:

$$
H_{l} \equiv \frac{4 \pi}{2 l+1} \sum_{m=-l}^{l}\left|\sum_{i} Y_{l}^{m}\left(\Omega_{i}\right) \frac{\left|p_{i}\right|}{E_{t o t}}\right|^{2}=\sum_{i, j} \frac{\left|p_{i}\right|\left|p_{j}\right|}{E_{t o t}^{2}} P_{l}\left(\cos \left(\phi_{i j}\right)\right),
$$

où les $Y_{l}^{m}(\Omega)$ sont les harmoniques sphériques et les $P_{l}(\cos (\phi))$ les polynômes de Legendre. $\phi_{i j}$ représente l'angle entre la particule $i$ et la particule $j$. Ces moments forment un échantillon complet de paramètres de forme. La conservation de l'énergie impulsion implique que $H_{0} \sim 1$ et $H_{1}=0$. Les événements de continuum $e^{+} e^{-} \rightarrow q \bar{q}$ ont une topologie en deux jets de directions opposées dans le référentiel du centre de masse des faisceaux, alors que les événements $B \bar{B}$ ont une topologie sphérique. En théorie, l'angle $\phi_{12}$ entre les deux jets (1 et 2 ) est donc égale à $\pi$. Or les polynômes de Legendre pris au point $\cos \left(\phi_{12}\right)$ avec $\phi_{12}=\pi$ sont égaux à 1 pour les $l$ pairs et à 0 pour les $l$ impaires. On a donc $H_{l}=1$ pour les $l$ pairs et $H_{l}=0$ pour les $l$ impairs et par conséquent, $R 2=1$.

On en déduit que la distribution de $R 2$ est plus proche de 1 pour les événements de continuum que pour les événements $B \bar{B}$. Cette variable permet ainsi de discriminer le continuum des événements $B \bar{B}$. Nous faisons la coupure $R 2<0,4$, dont l'efficacité est de $90 \%$ pour le signal et de $50 \%$ pour le continuum. La figure 5.10 montre que la coupure supprime une grande partie du continuum et peu de signal.

Une autre variable discriminante intéressante est l'angle $\psi$ entre le pion mou et la direction de vol du $D^{*}$ dans le référentiel du centre de masse du $D^{*}$. En pratique, pour déterminer cet angle, on suppose que $\phi=0$. Comme le $D^{*}$ a un spin 1 et une hélicité 0 , la distribution de $\psi$ du signal est proportionnelle à $\cos (\psi)^{2}$. Le signal pique donc à 


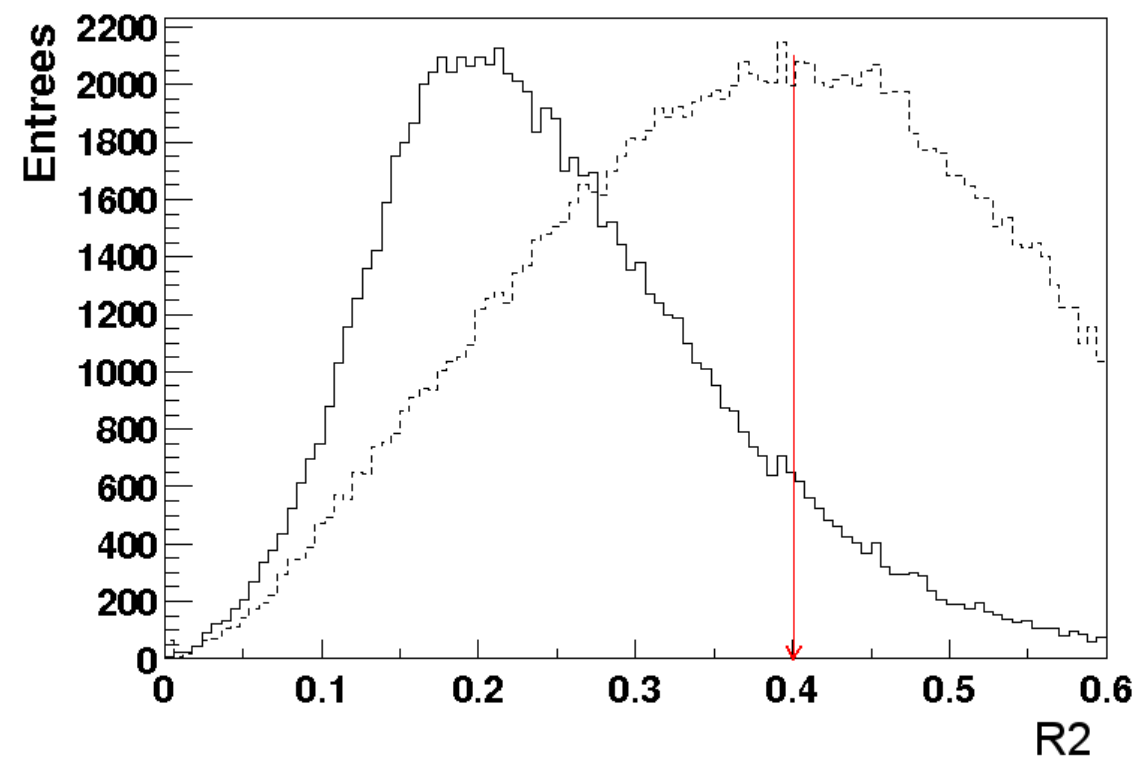

FIG. 5.10: Distribution de la variable R2 pour des événements de signal Monte-Carlo (ligne pleine) et de continuum provenant des données prises hors résonance (pointillés). On ne conserve que les événements à gauche de la flèche.

$|\cos (\psi)|=1$. On réalise la coupure $|\cos (\psi)|>0,4$, dont l'efficacité est de $81 \%$ pour le signal et de $66 \%$ pour le continuum. La distribution de $\cos (\psi)$ pour le signal et le continuum est représentée figure 5.11.

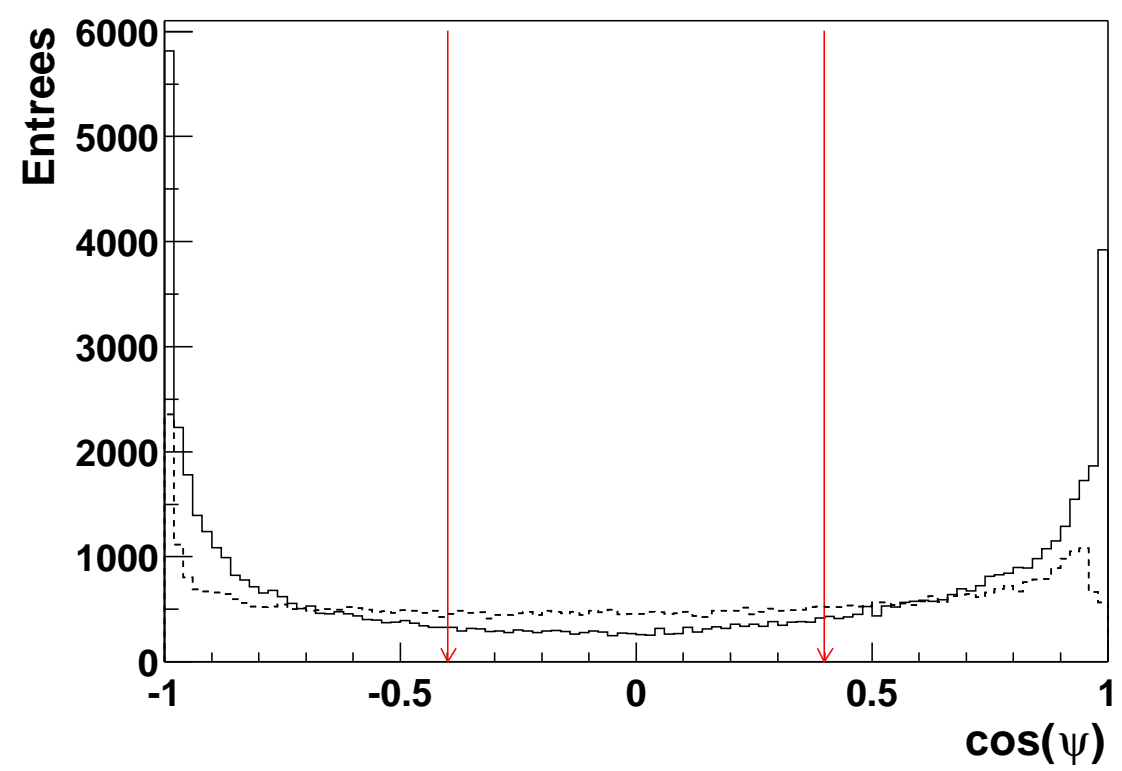

FIG. 5.11: Distribution de la variable $\cos (\psi)$ pour des événements de signal Monte-Carlo (ligne pleine) et de continuum provenant des données prises hors résonance (pointillés). On rejette les événements situés entre les deux flèches. 


\subsubsection{Sélection du meilleur candidat}

Après toutes ces coupures, on fait une sélection supplémentaire afin de trouver le meilleur candidat dans l'événement. On sélectionne ceux dont les paires de traces ont des charges opposées et on calcule la masse manquante définie par l'équation 5.2. S'il y a plusieurs candidats possibles, on choisit celui dont la masse de l'objet non reconstruit est la plus proche de la masse du $D^{0}$. On ne conserve que les événements tels que $1,81 \mathrm{GeV} / c<$ $m_{\text {rec }}<1,88 \mathrm{GeV} / c$.

Cette méthode permet de sélectionner un grand nombre d'événements de signal, mais également du bruit de fond.

\subsection{3 Échantillons de données utilisées}

\section{Données réelles}

L'analyse présentée ici utilise toutes les données enregistrées par BABAR jusqu'à l'été 2004, ce qui correspond à une luminosité intégrée de $211 \mathrm{fb}^{-1}$. Une partie des données a été enregistrée à $40 \mathrm{MeV}$ sous la résonance $\Upsilon(4 S)$ et est utilisée pour étudier le continuum. La luminosité de cet échantillon est de $21,7 \mathrm{fb}^{-1}$.

\section{Monte-Carlo}

Les événements de Monte-Carlo utilisés ont été générés avec GEANT 4. On utilise un échantillon d'événements de $B$ génériques correspondant à une luminosité de $666 \mathrm{fb}^{-1}$, soit environ 700 millions de paires $B \bar{B}$. Cette luminosité est environ trois fois plus importante que dans les données.

Trois échantillons d'événements simulés de signal (désintégration $\Upsilon(4 S) \rightarrow B \bar{B}$, suivie de $B \rightarrow D^{* \mp} \pi^{ \pm}$, suivie de $\left.D^{* \mp} \rightarrow D^{0} \pi^{\mp}\right)$ sont également utilisés avec différentes configurations :

1. Le signal $D^{*} \pi$ généré sans violation de $C P$, dont la luminosité intégrée correspondante est $687 \mathrm{fb}^{-1}$.

2. Le signal $D^{*} \pi$ généré avec violation de $C P$. Les paramètres utilisés pour la génération sont $2 \beta+\gamma=2,2, \delta=0,3$ et $r^{*}=0,025$. La valeur choisie pour générer $2 \beta+\gamma$ est proche de la valeur estimée par les contraintes que l'on a sur le triangle d'unitarité. La valeur générée pour $\delta$ est proche de la prédiction donnée par la factorisation naïve $(\delta=0$ ou $\pi)$. La luminosité de cet échantillon est $697 \mathrm{fb}^{-1}$.

3. Le signal $D^{*} \pi$ généré avec des asymétries de $C P$ amplifiées. La valeur utilisée pour $r^{*}$ est alors $r^{*}=0,10$. La luminosité de cet échantillon est $308 \mathrm{fb}^{-1}$.

Tous ces échantillons ont été générés avec un temps de vie du $B^{0} \tau=1,548$ ps, une fréquence de mélange $B^{0} \bar{B}^{0} \Delta m=0,489 \mathrm{ps}^{-1}$ et $\mathcal{B}\left(B^{0} \rightarrow D^{*-} \pi^{+}\right)=2,7.10^{-3}$.

\subsection{Procédure de l'analyse}

L'analyse a pour but de mesurer une asymétrie de $C P$ dépendante du temps dans les désintégrations $B \rightarrow D^{* \mp} \pi^{ \pm}$. 
L'analyse est basée sur une série d'ajustements par une méthode de maximum de vraisemblance. On ajuste simultanément les données prises à la résonance et hors résonance. Les données prises hors résonance ne contiennent que du continuum : l'ajustement simultané sur les données à la résonance et hors résonance permet de mieux contraindre les paramètres du continuum. On définit une fonction densité de probabilité (notée PDF dans la suite) qui dépend de plusieurs variables : la masse manquante $m_{\text {rec }}, \Delta t$, l'erreur sur $\Delta t$ notée $\sigma_{\Delta t}$, un discriminant de Fisher $F$, ainsi que de deux variables $S_{\text {tag }}$ et $S_{\text {mix }}$ :

- $S_{\text {tag }}$ dépend de la saveur de $B_{\text {tag. }}$. Par convention $S_{\text {tag }}=1$ si $B_{\text {tag }}$ est identifié comme un $B^{0}$ et $S_{\text {tag }}=-1$ si $B_{\text {tag }}$ est identifié comme un $\bar{B}^{0}$.

- $S_{\text {mix }}$ vaut 1 pour un événement qui s'est "mélangé" et -1 pour un événement qui ne s'est pas "mélangé".

Ici, le terme "mélangé" ne correspond pas exactement à la définition physique, mais plutôt à une définition expérimentale. En négligeant les désintégrations défavorisées $B^{0} \rightarrow$ $D^{*+} \pi^{-}$et $\bar{B}^{0} \rightarrow D^{*-} \pi^{+}$, on considère que si on reconstruit un pion dur de charge positive (négative), celui-ci est issu d'un $B^{0}\left(\bar{B}^{0}\right)$ par la désintégration $B^{0} \rightarrow D^{*-} \pi^{+}\left(\bar{B}^{0} \rightarrow\right.$ $\left.D^{*+} \pi^{-}\right)$, et par conséquent, $B_{\text {rec }}$ est un $B^{0}\left(\bar{B}^{0}\right)$. D'autre part, on connaît la saveur de $B_{\text {tag }}$ grâce à l'étiquetage. On appelle "mélangé", dans cette analyse, un événement avec $\operatorname{deux} B$ de la même saveur, c'est-à-dire un événement dans lequel $B_{\text {tag }}$ est un $B^{0}$ et le pion dur a une charge positive ou dans lequel $B_{\text {tag }}$ est un $\bar{B}^{0}$ et le pion dur a une charge négative.

La PDF utilisée pour les données sous la résonance est la somme pondérée des PDFs de chaque catégorie d'événement : le signal $D^{*} \pi$, le bruit de fond $D^{*} \rho$, le bruit de fond qui pique, le combinatoire et le continuum :

$$
P=\sum_{i} f_{i} P_{i}
$$

où l'indice $i$ désigne les différentes catégories d'événements que l'on notera dans la suite $D^{*} \pi, D^{*} \rho$, peak, comb et $q \bar{q} . f_{i}$ représente la fraction de chaque catégorie d'événement, et $P_{i}$ est la PDF correspondante. Chacune de ces PDFs est le produit de trois PDFs. :

$$
\mathcal{P}_{i}\left(m_{\text {rec }}, F, \Delta t, \sigma_{\Delta t}, S_{\text {tag }}, S_{\text {mix }}\right)=\mathcal{M}_{i}(\mathrm{~m}) \mathcal{F}_{\mathrm{i}}(\mathrm{F}) \mathcal{T}_{\mathrm{i}}^{\prime}\left(\Delta \mathrm{t}, \sigma_{\Delta \mathrm{t}}, \mathrm{S}_{\mathrm{tag}}, \mathrm{S}_{\text {mix }}\right), .
$$

$\mathcal{M}_{i}$ est la PDF de la masse manquante $m_{\mathrm{rec}}, \mathcal{F}_{i}$ la PDF du discriminant de Fisher $F$ et $\mathcal{T}^{\prime} i$ la PDF de $\Delta t$. Ces différentes PDFs sont détaillées dans la suite.

On cherche à ajuster les paramètres de la PDF totale de façon à ce que la PDF reproduise au mieux la distribution des événements des données pour les différentes variables. Pour cela, on dispose d'un échantillon de $n$ événements $\left\{\vec{x}_{k}\right\}_{k=1}^{n} \cdot \vec{x}_{k}$ représente les variables de l'événement $k$. Les variables sont celles de la PDF : $\Delta t, m_{\text {rec }}, F, S_{\text {tag }}$ et $S_{\text {mix }}$. La PDF est paramétrée par un vecteur de paramètres $\vec{p}$, que l'on veut ajuster. Pour cela, on construit une quantité $\mathcal{L}$, appelée vraisemblance :

$$
\mathcal{L}(\vec{p})=\sum_{k=1}^{n} \operatorname{PDF}\left(\vec{x}_{k}, \vec{p}\right) .
$$

La PDF doit être positive et normalisée. La normalisation de la PDF se traduit de la façon suivante : 


$$
\frac{\partial}{\partial p_{k}} \int d x_{1} \ldots \int d x_{n} P D F\left(\vec{x}_{k}, \vec{p}\right) \theta(\vec{x})=0
$$

Dans cette équation, $\theta(\vec{x})$ représente l'ensemble des coupures appliquées aux variables avant l'ajustement. Il en résulte des facteurs de normalisation dans la PDF.

$\mathcal{L}$ quantifie la vraisemblance de l'ajustement. On cherche donc à maximiser cette quantité en ajustant les différents paramètres de la PDF entre leurs limites. On réalise ainsi un ajustement par maximum de vraisemblance, en utilisant le logiciel MINUIT [84].

L'analyse comporte quatre étapes principales:

1. L'ajustement des variables cinématiques, décrit dans la partie 5.4.

2. L'ajustement des paramètres $\alpha$ et $\rho$ du signal, décrit dans la partie 5.4.5.

3. L'ajustement des paramètres de $\mathcal{T}_{\text {comb }}$ et de la fonction de résolution du combinatoire : on utilise alors les données prises dans une bande latérale en $m_{\text {rec }}$ par rapport à la région du signal. Cela correspond à la région $1,81 \mathrm{GeV} / c^{2}<m_{\mathrm{rec}}<1,84 \mathrm{GeV} / c^{2}$, dans laquelle il n'y a que du bruit de fond combinatoire et continuum. On réalise un ajustement simultané sur les données prises à la résonance $\Upsilon(4 S)$ et hors résonance. Cela permet d'ajuster à la fois les paramètres du combinatoire et du continuum.

4. Ajustement final dans la région du signal $\left(1,845 \mathrm{GeV} / c^{2}<m_{\text {rec }}<1,88 \mathrm{GeV} / c^{2}\right)$. On ajuste les paramètres du signal et du continuum sur les données à la résonance et hors résonance simultanément, en fixant les paramètres ajustés dans les étapes précédentes. Les paramètres du bruit de fond qui pique sont déterminés sur Monte Carlo.

La partie 5.5 décrit la procédure de l'analyse en $\Delta t$ des événements de signal. La partie 5.6 décrit le traitement des différents bruits de fond et leur impact sur l'ajustement dans la région du signal. Les résultats de l'ajustement sur les données sont présentés dans la partie 5.7.

\subsection{Ajustement des variables cinématiques}

Cette première étape de l'analyse est un ajustement des distributions de $F$ et $m_{\text {rec }}$ réalisé sur l'ensemble des données telles que $1.81<m_{\text {rec }}<1.88 \mathrm{GeV}$. L'ajustement se fait simultanément sur les données sous et hors résonance, et ce afin de bien décrire les distributions de continuum. Il n'est cependant pas possible d'ajuster les paramètres de tous les types de bruits de fond considérés dans cette analyse.

La corrélation entre les deux variables $F$ et $m_{\text {rec }}$ a été calculée sur Monte Carlo pour différents types d'événements : signal, bruit de fond provenant de la désintégration de $\stackrel{(}{B}$ (noté bdf $(\bar{B})$ ou de $B^{ \pm}$(bdf $B^{ \pm}$) et continuum. Les coefficients de correlation sont montrés dans le tableau 5.1.

Les corrélations entre $F$ et $m_{\text {rec }}$ sont de l'ordre du $\%$ : on peut donc les négliger et utiliser une PDF qui est le produit de deux PDFs à une dimension $(\mathcal{M}$ et $\mathcal{F})$. 


\begin{tabular}{|c|c|}
\hline signal & $-0,028$ \\
bdf $\stackrel{(\vec{B}}{\text { bdf } B^{ \pm}}$ & $-0,037$ \\
$-0,023$ \\
continuum & 0,005 \\
\hline
\end{tabular}

TAB. 5.1: Corrélation entre $F$ et $m_{\text {rec }}$ pour différents types d'événements (Monte Carlo)

\subsubsection{PDF $\mathcal{M}$, décrivant la distribution de $m_{\text {rec }}$}

Pour chaque type d'événement, la PDF en masse manquante est la somme d'une gaussienne "bifurquée" $\mathcal{B G}$ et d'une fonction ARGUS $\mathcal{A}$ :

$$
\mathcal{M}_{i}\left(m_{\mathrm{rec}}\right)=f_{b} \mathcal{B \mathcal { G }}_{i}\left(m_{\mathrm{rec}}\right)+\left(1-f_{b}\right) \mathcal{A}_{i}\left(m_{\mathrm{rec}}\right),
$$

$f_{b}$ représente la fraction de gaussienne "bifurquée".

Une gaussienne "bifurquée" est une gaussienne qui n'a pas la même largeur à droite et à gauche de sa valeur centrale. Cette fonction permet de bien modéliser les événements qui piquent dans la région du signal. Son expression est :

$$
\mathcal{B G}_{i}\left(m_{\text {rec }}\right) \propto \begin{cases}\exp \left[-\left(m_{\text {rec }}-\mu_{i}\right)^{2} / 2\left(\sigma_{i}^{L}\right)^{2}\right], & m_{\text {rec }}<\mu_{i} \\ \exp \left[-\left(m_{\text {rec }}-\mu_{i}\right)^{2} / 2\left(\sigma_{i}^{R}\right)^{2}\right], & m_{\text {rec }} \geq \mu_{i}\end{cases}
$$

$\mu_{i}$ est la valeur centrale où se situe le pic de la fonction et $\sigma_{i}^{R}$ et $\sigma_{i}^{L}$ représentent les largeurs droite et gauche respectivement.

La fonction ARGUS est une fonction développée par la collaboration ARGUS [85] : elle permet de bien modéliser la partie plate de la distribution et possède l'avantage d'avoir un point d'arrêt. Son expression est la suivante :

$$
\mathcal{A}\left(m_{\mathrm{rec}}\right) \propto \begin{cases}m_{\mathrm{rec}} \sqrt{1-\left(m_{\mathrm{rec}} / M_{i}\right)^{2}} \exp \left[\epsilon_{i}\left(1-\left(m_{\mathrm{rec}} / M_{i}\right)^{2}\right)\right], & m_{\mathrm{rec}}<M_{i} \\ 0, & m_{\mathrm{rec}} \geq M_{i}\end{cases}
$$

où $M_{i}$ représente le point d'arrêt.

Les distributions de $m_{\text {rec }}$ sont ajustées par la somme d'une fonction ARGUS et d'une gaussienne bifurquée. Les différentes catégories d'événements ont des distributions différentes en $m_{\text {rec }}$. On utilise une PDF pour tout le continuum, une PDF pour le bruit de fond combinatoire, une PDF pour le bruit de fond qui pique en $m_{\text {rec }}$, sans distinguer les désintégrations des $B^{0}$ des désintégrations des $B^{+}$, une PDF pour le bruit de fond $B^{0} \rightarrow D^{*-} \rho^{+}$et une PDF pour le signal. Les formes de ces différentes PDFs ont été ajustées sur Monte-Carlo.

\subsubsection{PDF $\mathcal{F}$, décrivant la distribution de $F$}

La PDF du discriminant de Fisher de chaque catégorie d'événement est la somme de deux gaussiennes.

Cependant, ainsi que cela a été dit dans la partie 5.1.3, le Fisher permet principalement de discriminer les événements $B \bar{B}$ du continuum. Des tests de Kolmogorov-Smirnov 
réalisés sur Monte-Carlo ont montré que les distributions de $F$ pour différents types d'événements $B \bar{B}$ étaient en raisonnable accord. En particulier, on a testé la compatibilité entre les distributions de $F$ du signal par rapport au bruit de fond $B^{0}$, du signal par rapport au bruit de fond $B^{+}$et du bruit de fond $B^{0}$ par rapport au bruit de fond $B^{+}$. Les résultats des tests de Kolmogorov-Smirnov correspondant sont résumés dans le tableau 5.2 pour les deux catégories d'étiquetage.

\begin{tabular}{|l|c|c|}
\hline type d'evt & étiquetage lepton & étiquetage kaon \\
\hline signal vs $B^{0}$ & 59.3 & 31.9 \\
signal vs $B^{+}$ & 2.9 & 27.2 \\
$B^{0}$ vs $B^{+}$ & 6.6 & 44.9 \\
\hline
\end{tabular}

TAB. 5.2: Résultats des tests de Kolmogorov-Smirnov (en \%) pour les distributions de $F$ de différents types d'événements $B \bar{B}$.

La bonne compatibilité entre ces différentes distributions nous conduit à utiliser une PDF commune à tous les événements $B \bar{B}$ pour $\mathcal{F}$. En revanche, la compatibilité entre les distributions de $F$ pour des événements étiquetés par des kaons ou des leptons est inférieure à $10^{-3}$. On ajuste donc les distributions de $F$ séparément pour les deux types d'étiquetage.

\section{Traitement du continuum}

On distingue deux catégories de continuum : les événements avec des quarks $u, d$, ou $s$ (notés $u d s$ dans la suite) et les événements avec des quarks $c$ (notés $c \bar{c}$ ). Ces deux catégories ont des distributions en $\Delta t, m_{\text {rec }}$ et $F$ différentes. Cependant, elles ne sont pas différenciées dans l'analyse. Cette approximation est motivée par un souci de simplicité. En effet, différencier ces deux catégories d'événements de continuum augmenterait le nombre de paramètres à ajuster, lesquels seraient très corrélés, car il n'y a pas de variable réellement discriminante pour séparer ces types d'événements.

L'effet de cette approximation sur la mesure des paramètres de $C P$ a été estimé à l'aide de simulations rapides. On a alors généré rapidement des événements de signal et de bruit de fond $B \bar{B}$, ainsi que des événements de continuum en distinguant les événements uds des événements $c \bar{c}$. On a ainsi simulé des événements à la résonance et hors résonance. Enfin, on a ajusté les distributions ainsi simulées avec deux PDFs pour modéliser le continuum, puis avec une seule PDF et on a étudié l'effet sur l'ajustement des paramètres de $C P$. Dans l'étude réalisée, on a supposé que la phase forte relative $\delta_{D^{*} \pi}$ était nulle et qu'il n'y avait pas de violation de $C P$ du côté du $B_{\text {tag }}\left(r^{\prime}=0\right)$. Cette hypothèse n'a pas d'impact sur l'étude. Le paramètre de $C P$ que l'on a mesuré pour cette étude est $\sin (2 \beta+\gamma)$. Pour chaque simulation rapide, on ajuste $\sin (2 \beta+\gamma)$ en modélisant le continuum avec deux PDF, puis on ajuste $\sin (2 \beta+\gamma)$ en modélisant le continuum avec une seule PDF. Afin d'estimer l'impact de cette approximation sur la mesure de $\sin (2 \beta+\gamma)$, il faut regarder la différence $\Delta \sin (2 \beta+\gamma)$ des valeurs ajustées de $\sin (2 \beta+\gamma)$ entre les deux ajustements. Les résultats sont représentés figure 5.12 .

L'effet de cette approximation est de l'ordre de 0,003 , ce qui est très faible par rapport à $\sin (2 \beta+\gamma)$, dont l'erreur est de l'ordre de 1 . 


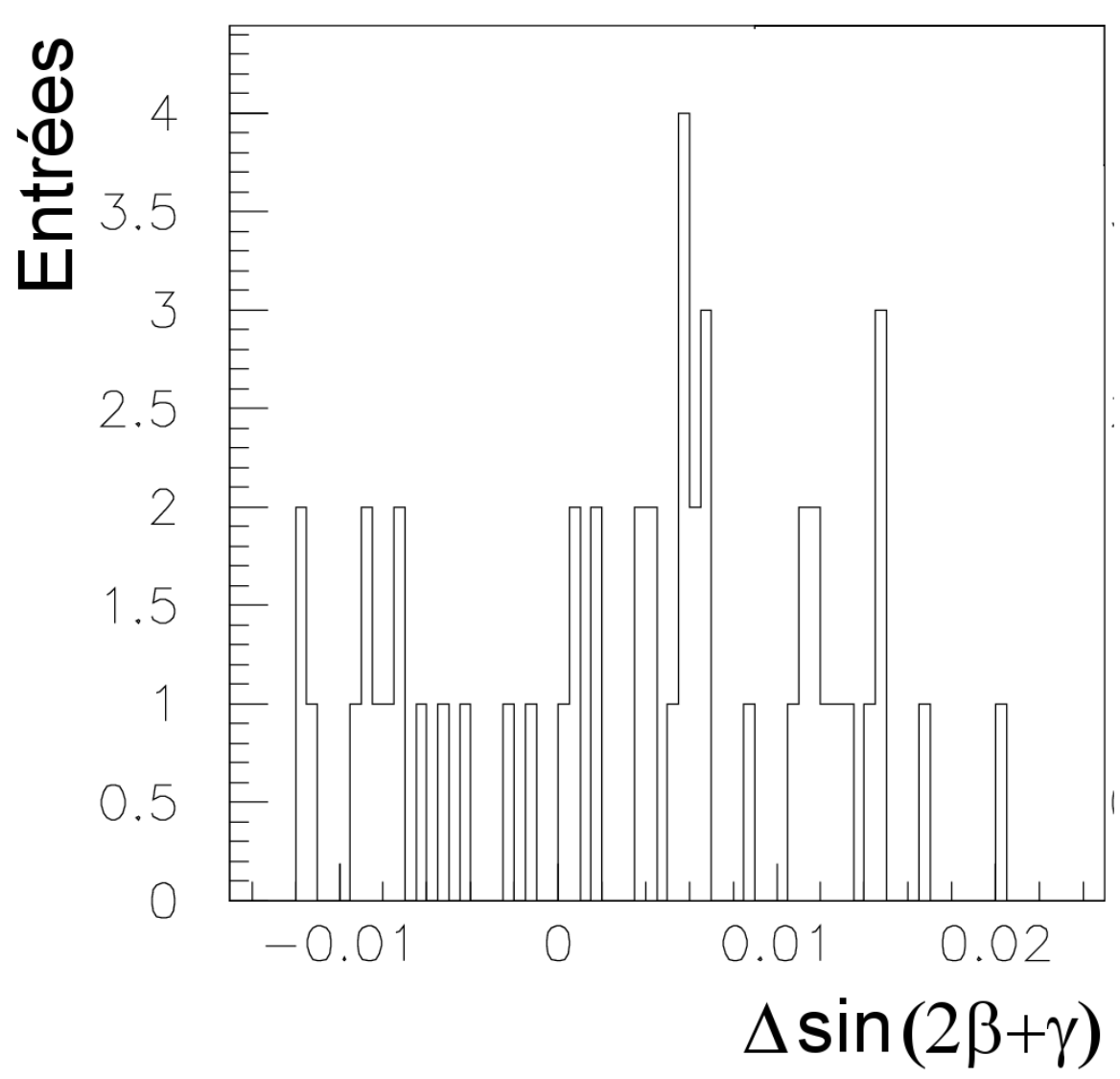

FIG. 5.12: Différence entre les valeurs de $\sin (2 \beta+\gamma)$ mesurées avec une et deux PDFs pour le continuum.

\subsubsection{Ajustement total des variables cinématiques}

On ajuste simultanément sur les données les distributions de $F$ et de $m_{\text {rec }}$, pour des valeurs de $m_{\text {rec }}$ comprises entre $1.81 \mathrm{GeV} / c^{2}$ et $1.88 \mathrm{GeV} / c^{2}$. Cet intervalle englobe la région latérale et la région du signal. Tous les paramètres des PDFs sont déterminés sur Monte-Carlo, sauf les paramètres suivants, ajustés sur les données :

- tous les paramètres de $\mathcal{F}$, pour le $B \bar{B}$ et le continuum

- les paramètres relatifs à la fonction ARGUS de $\mathcal{M}$ pour le continuum

- les largeurs de la gaussienne bifurquée de $\mathcal{M}$ du signal.

Cet ajustement permet également de déterminer les fractions des différents types d'événements. On détermine la fraction d'événements $B \bar{B}$ en utilisant les nombres d'événements hors-résonance, qui ne sont a fortiori que du continuum, et le rapport entre la luminosité intégrée à la résonance et hors-résonance. La fraction de $B \bar{B}$ est ainsi donnée par :

$$
f_{B \bar{B}}=1-\frac{N_{o f f}}{N_{\text {on }}} \frac{\mathcal{L}_{\text {on }}}{\mathcal{L}_{\text {off }}}
$$

où $N_{\text {on }}$ et $N_{\text {off }}$ représentent respectivement le nombre d'événements pris à la résonance et hors résonance et $\mathcal{L}_{o n}$ et $\mathcal{L}_{\text {off }}$ les luminosités intégrées à la résonance et hors résonance. 
Il n'est pas possible de déterminer de façon fiable la fraction d'événements $D^{*} \pi$ par rapport à l'ensemble des événements $D^{*} \pi$ et $D^{*} \rho$. On fixe donc cette fraction à une valeur déterminée grâce aux rapports d'embranchement du PDG [29] et qui tient compte de l'efficacité de sélection sur Monte-Carlo des deux types d'événements. On a ainsi $f_{D^{*} \pi}=$ $0,87 \pm 0,03$.

La fraction de $\left(D^{*} \pi+D^{*} \rho\right)$ par rapport à l'ensemble des $B \bar{B}$, ainsi que la fraction de bruit de fond combinatoire par rapport à l'ensemble du bruit de fond combinatoire et qui pique sont ajustées sur les données.

\subsubsection{Résultats de l'ajustement des variables cinématiques}

L'ajustement correspondant à l'étape 1 de l'analyse a été réalisé sur les données. Les valeurs des paramètres ajustés sont résumées dans le tableau 5.3.

La PDF du Fisher est la somme de deux gaussiennes : $b_{I}^{1}$ et $b_{I}^{2}$ sont les biais, $s_{I}^{1}$ et $s_{I}^{2}$ sont les largeurs et $f_{I}$ est la fraction de la première gaussienne. Ici, $I$ fait référence au type d'événement $I . \epsilon^{m_{\mathrm{rec}}, q \bar{q}}$ et $f_{b}^{m_{\mathrm{rec}}, q \bar{q}}$ sont le coefficient de l'exponentielle de la fonction ARGUS et la fraction de gaussienne bifurquée pour la PDF $\mathcal{M}$ du continuum. $f_{\rho+\pi}$ est la fraction d'événements $D^{*} \pi$ ou $D^{*} \rho$ parmi les événements $B \bar{B}$ et $f_{\text {comb }}$ est la fraction de combinatoire parmi les événements $B \bar{B}$ autres que $D^{*} \pi$ et $D^{*} \rho$.

\begin{tabular}{|l|c|c|}
\hline Variable & étiquetage lepton & étiquetage kaon \\
\hline$b_{B \bar{B}}^{1}$ & $-0,169 \pm 0,022$ & $-0,281 \pm 0,009$ \\
$b_{B \bar{B}}^{2}$ & $-0,502 \pm 0,004$ & $-0,603 \pm 0,003$ \\
$f_{B \bar{B}}$ & $0,289 \pm 0,026$ & $0,474 \pm 0,016$ \\
$s_{B \bar{B}}^{1}$ & $0,287 \pm 0,007$ & $0,263 \pm 0,003$ \\
$s_{B \bar{B}}^{2}$ & $0,203 \pm 0,002$ & $0,187 \pm 0,001$ \\
$b_{q \bar{q}}^{1}$ & $-0,018 \pm 0,017$ & $0,201 \pm 0,051$ \\
$b_{q \bar{q}}^{2}$ & $-0,187 \pm 0,021$ & $-0,098 \pm 0,003$ \\
$f_{q \bar{q}}$ & $0,656 \pm 0,084$ & $0,050 \pm 0,014$ \\
$s_{q \bar{q}}^{1}$ & $0,382 \pm 0,007$ & $0,460 \pm 0,012$ \\
$s_{q \bar{q}}^{2}$ & $0,237 \pm 0,027$ & $0,327 \pm 0,002$ \\
\hline$f_{\rho+\pi}$ & $0,381 \pm 0,007$ & $0,322 \pm 0,003$ \\
$f_{\text {comb }}$ & $0,915 \pm 0,018$ & $0,924 \pm 0,007$ \\
$\epsilon_{\text {rec }}^{m_{\bar{q}}}, q \bar{q}$ & $-8,03 \pm 0,83$ & $-10,03 \pm 0,28$ \\
$f_{b}^{m_{\text {rec }}, q \bar{q}}$ & $9.10^{-8} \pm 4.10^{-3}$ & $0,00052 \pm 0,00001$ \\
$f_{B \bar{B}}$ & 0,818 & 0,392 \\
\hline
\end{tabular}

TAB. 5.3: Résultats de l'ajustement cinématique sur les données

Il y a $18705 \pm 273$ événements de signal $D^{*} \pi$ étiquetés par un lepton et $70534 \pm$ 661 événements de signal $D^{*} \pi$ étiquetés par un kaon. Les fractions des différents types d'événements dans la région du signal sont récapitulées dans le tableau 5.4. Ces fractions sont calculées à partir des fractions déterminées sur l'ensemble des événements, en utilisant les intégrales des PDFs $\mathcal{M}$ des différents types de bruits de fond dans la région du signal. 


\begin{tabular}{|l|c|c|}
\hline fraction & étiquetage lepton & étiquetage kaon \\
\hline$f_{D^{*} \pi}$ & 0,45 & 0,23 \\
$f_{D^{*} \rho}$ & 0,07 & 0,04 \\
$f_{\text {comb }}$ & 0,30 & 0,20 \\
$f_{\text {peak }}$ & 0,06 & 0,03 \\
$f_{q \bar{q}}$ & 0,12 & 0,50 \\
\hline
\end{tabular}

TAB. 5.4: Fractions des différents types d'événements dans la région du signal $\left(1,845 \leq m_{\mathrm{rec}} \leq\right.$ $\left.1,88 \mathrm{GeV} / c^{2}\right)$.

Les figures des distributions en $m_{\text {rec }}$ et en $F$ pour les deux catégories d'événements sont montrées figures 5.13 et 5.14 .

\subsubsection{Détermination des paramètres $\alpha$ et $\rho$}

L'ajustement des variables cinématiques permet également de déterminer la fraction $\alpha$ d'événements étiquetés par une trace du $D^{0}$ non reconstruit, ainsi que la fraction $\rho$ de ces événements qui correspond à des événements mélangés.

On a vu dans la partie 5.1.4 que l'on ne sélectionnait que les événements tels que $\cos \left(\theta_{\text {tag }}\right)<0,75(0,50)$ pour les événements étiquetés par un lepton (kaon), afin de limiter la fraction d'événements étiquetés par une trace du $D^{0}$ non reconstruit. On définit les quantités $N_{p}^{q}$, où $p=A L L, M I X E D$ ou $U N M I X E D$ et $q=l$ ou $g$. Ces quantités représentent les nombres d'événements de signal déterminés par l'ajustement des variables cinématiques décrit plus haut pour différents échantillons d'événements. L'indice $q=l$ $(q=g)$ signifie que l'on considère des événements tels que $\cos \left(\theta_{\text {tag }}\right)<0,75(>0,50)$. L'indice $M I X E D$ signifie que l'on ne considère que les événements mélangés, l'indice UNMIXED signifie que l'on ne considère que les événements non mélangés et l'indice $A L L$ signifie qu'on considère tous les événements, mélangés ou non. On a également besoin de connaître la fraction $\epsilon^{B}\left(\epsilon^{D}\right)$ de traces étiquetées par un $B\left(D^{0}\right)$ qui satisfont le critère $\cos \left(\theta_{\text {tag }}\right)<0,75$ pour les événements étiquetés par un lepton ou $\cos \left(\theta_{\text {tag }}\right)<0,50$ pour les événements étiquetés par un kaon. Ces fractions sont déterminées grâce aux distributions de signal Monte-Carlo représentées figures 5.6 et 5.7.

On appelle $\alpha^{\prime}$ la fraction totale d'événements étiquetés par une trace du $D^{0}$, sans coupure sur $\cos \theta_{\text {tag. }}$. Par définition, on a :

$$
N^{l}=\left(N^{l}+N^{g}\right)\left(\left(1-\alpha^{\prime}\right) \epsilon^{B}+\alpha^{\prime} \epsilon^{D}\right) .
$$

Avec $f^{l}=\frac{N^{l}}{N^{l}+N^{g}}$, on obtient :

$$
\alpha^{\prime}=\frac{\epsilon^{B}-f^{l}}{\epsilon^{B}-\epsilon^{D}}
$$

On en déduit alors $\alpha$ en utilisant la relation :

$$
\alpha=\frac{\epsilon^{D} \alpha^{\prime}}{\left(1-\alpha^{\prime}\right) \epsilon^{B}+\alpha^{\prime} \epsilon^{D}} .
$$

De la même façon, on peut écrire : 

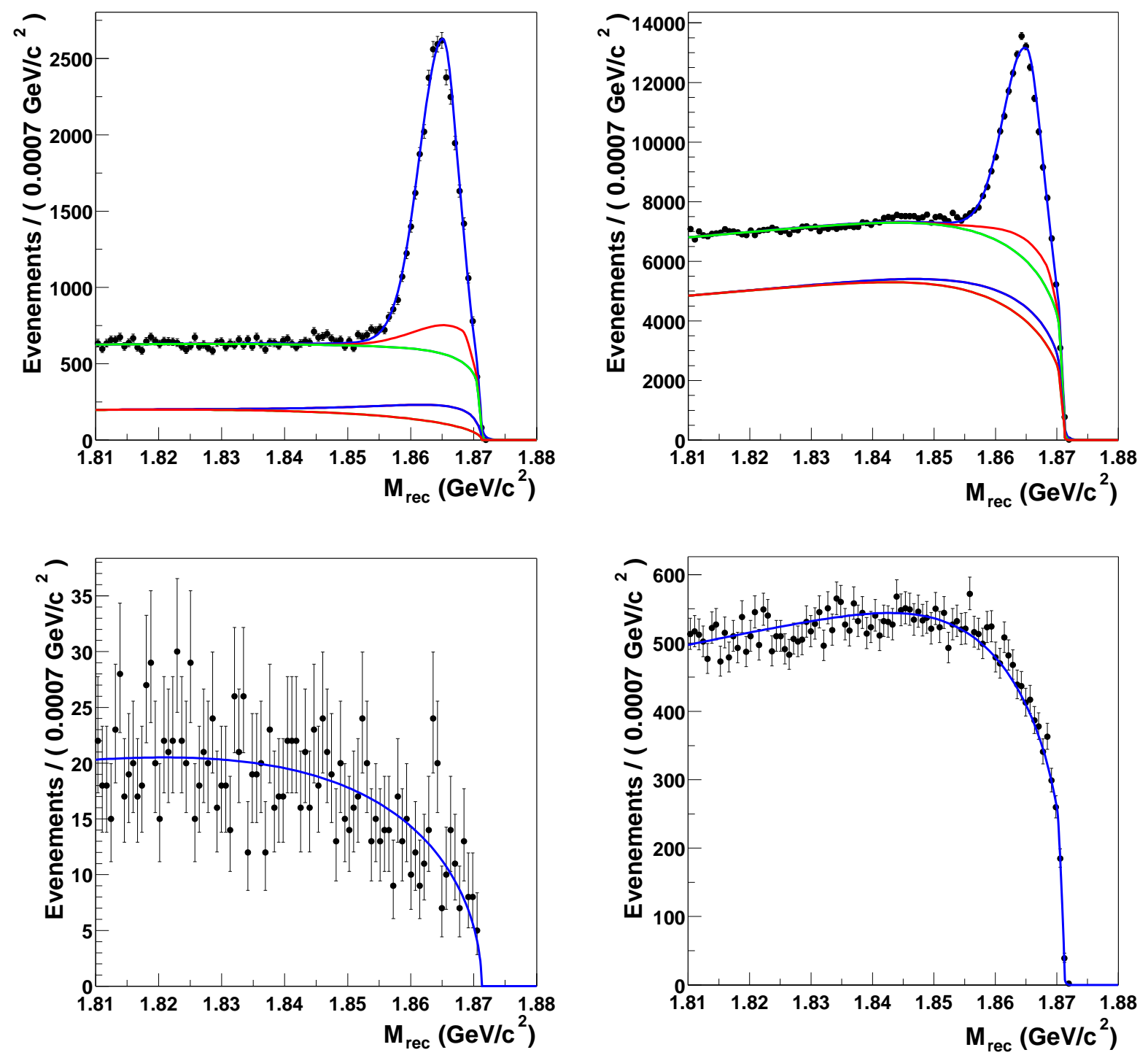

FIG. 5.13: Distribution en $m_{\text {rec }}$ pour les données prises à la résonance (haut) et hors résonance (bas) pour des événements étiquetés par un lepton (gauche) et pour des événements étiquetés par un kaon (droite). Les courbes montrent, de bas en haut, les contributions cumulées du continuum, du bruit de fond qui pique, du combinatoire, du bruit de fond $D^{*} \rho$ et du signal $D^{*} \pi$. 

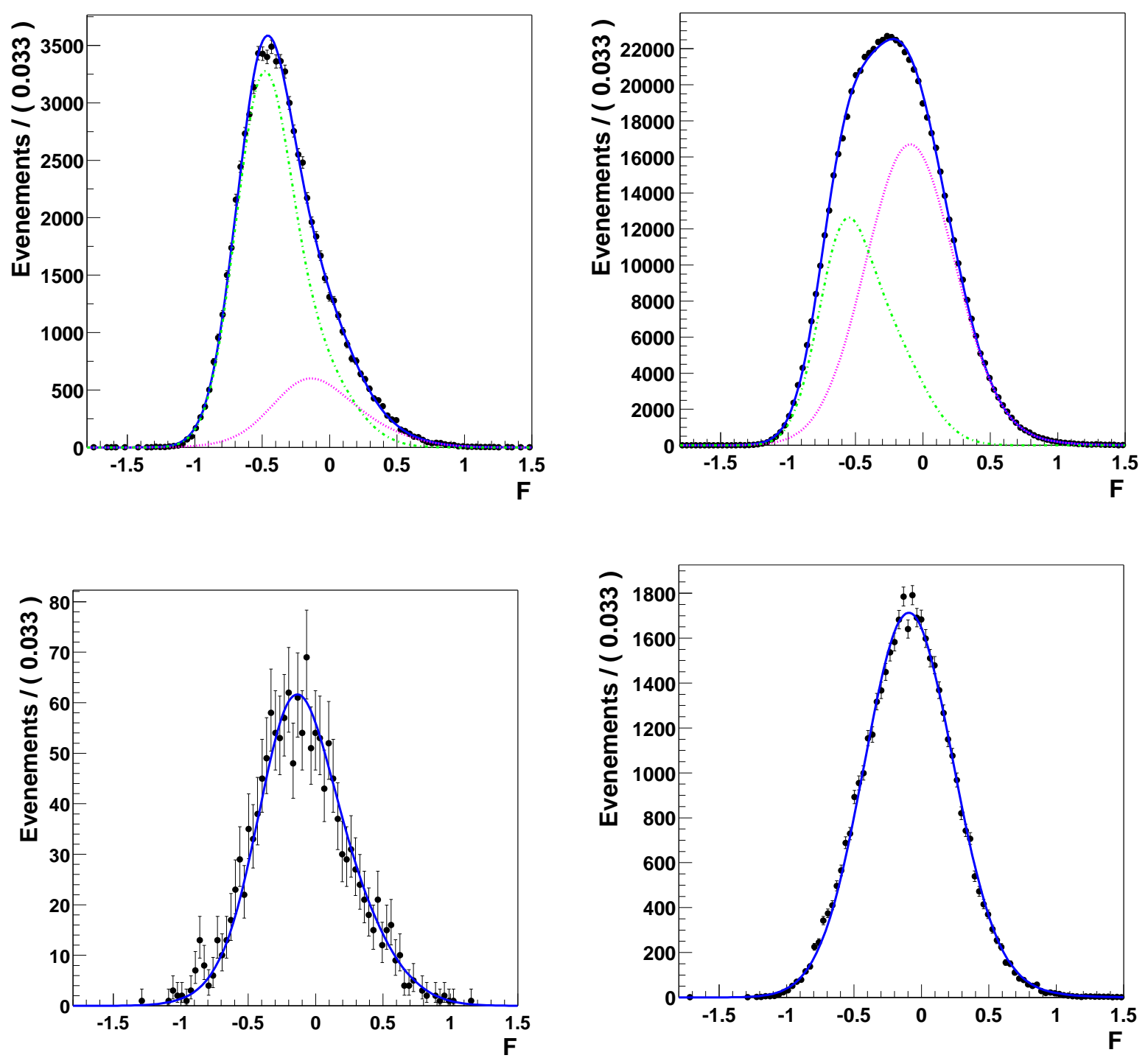

FIG. 5.14: Distribution en $F$ pour les données prises à la résonance (haut) et hors résonance (bas) pour des événements étiquetés par un lepton (gauche) et pour des événements étiquetés par un kaon (droite). Les contributions des événements $B \bar{B}$ (ligne en tiretés, piquant à environ -0,6) et du continuum (ligne en pointillés, piquant à environ -0,1) sont superposées. La PDF totale (en trait plein) est également présentée. 


$$
\begin{aligned}
& N_{M I X}^{g}=N^{B}\left(1-\epsilon^{B}\right)+N^{D} \rho\left(1-\epsilon^{D}\right), \\
& N_{M I X}^{l}=N^{B} \epsilon^{B}+N^{D} \rho \epsilon^{D}
\end{aligned}
$$

où $N^{D}=\left(N^{l}+N^{g}\right) \alpha$ et $N^{B}$ est le nombre total d'événements mélangés avec une trace étiquetante provenant du $B_{\mathrm{tag}}$. On détermine également $\rho$ de la façon suivante :

$$
\rho=\frac{\epsilon^{B} N_{M I X}^{g}-\left(1-\epsilon^{B}\right) N_{M I X}^{l}}{N^{D}} \frac{1}{\epsilon^{B}\left(1-\epsilon^{D}\right)-\left(1-\epsilon^{B}\right) \epsilon^{D}} .
$$

De la même façon, on peut déduire $1-\rho$ en utilisant les événements non mélangés. 


\subsubsection{Résultats de l'ajustement de $\alpha$ et $\rho$}

\section{Événements étiquetés par un lepton}

Les valeurs des nombres d'événements $N_{p}^{q}$ sont résumées dans le tableau 5.5.

\begin{tabular}{|c|c|c|}
\hline & $\cos \left(\theta_{\mathrm{tag}}\right)<0,75$ & $\cos \left(\theta_{\mathrm{tag}}\right)>0,75$ \\
\hline ALL & $19144 \pm 242$ & $5041 \pm 186$ \\
UNMIXED & $14452 \pm 212$ & $4273 \pm 159$ \\
MIXED & $4664 \pm 116$ & $586 \pm 90$ \\
\hline
\end{tabular}

TAB. 5.5: Valeurs des nombres d'événements $N_{p}^{q}$ pour les événements étiquetés par un lepton

On déduit de ces valeurs que $\alpha=0,0105 \pm 0,0005$ et $\rho=0,09 \pm 0,02$.

\section{Événements étiquetés par un kaon}

Les valeurs des nombres d'événements $N_{p}^{q}$ sont résumées dans le tableau 5.6.

\begin{tabular}{|c|c|c|}
\hline & $\cos \left(\theta_{\mathrm{tag}}\right)<0,50$ & $\cos \left(\theta_{\mathrm{tag}}\right)>0,50$ \\
\hline$A L L$ & $70584 \pm 661$ & $8392 \pm 201$ \\
$U N M I X E D$ & $45769 \pm 343$ & $6826 \pm 186$ \\
$M I X E D$ & $23587 \pm 358$ & $1265 \pm 70$ \\
\hline
\end{tabular}

TAB. 5.6: Valeurs des nombres d'événements $N_{p}^{q}$ pour les événements étiquetés par un kaon

On déduit de ces valeurs que $\alpha=0,055 \pm 0,001$ et $\rho=0,92 \pm 0,03$.

\section{Conclusion}

On remarque que parmi les événements étiquetés par un lepton, très peu (environ $1 \%$ ) sont étiquetés par une trace du $D^{0}$ non reconstruit, alors que pour les événements étiquetés par un kaon, la fraction d'événements étiquetés par une trace du $D^{0}$ est plus importante (environ 5,5\%). Cela s'explique par le fait que l'étiquetage par un lepton est plus propre et il est plus facile dans ce cas-là d'éviter de sélectionner une trace provenant du $D^{0}$ non reconstruit en utilisant une simple coupure sur $\cos \left(\theta_{\mathrm{tag}}\right)$. 


\subsection{Analyse en $\Delta t$ pour le signal $D^{*} \pi$}

\subsubsection{Introduction sur l'analyse en $\Delta t$}

La PDF dépendant de $\Delta t, \mathcal{T}^{\prime}$, que nous utilisons est la convolution de la PDF qui dépend de $\Delta t_{\text {vrai }}, \mathcal{T}$ et de la fonction de résolution $\mathcal{R}$ :

$$
\mathcal{T}_{i}^{\prime}\left(\Delta t, \sigma_{\Delta t}, S_{\text {tag }}, S_{\text {mix }}\right)=\int d \Delta t_{\text {vrai }} \mathcal{T}_{i}\left(\Delta t_{\text {vrai }}, S_{\text {tag }}, S_{\text {mix }}\right) \mathcal{R}_{i}\left(\Delta t-\Delta t_{\text {vrai }}, \sigma_{\Delta t}\right)
$$

On appelle $\Delta t_{\text {vrai }}$ la mesure que l'on aurait faite avec un détecteur parfait. Il faut cependant tenir compte de la connaissance imparfaite que l'on a du détecteur, ainsi que de la résolution intrinsèque de celui-ci. Pour cela, on convolue la PDF avec une fonction de résolution qui est décrite plus loin.

On distingue deux catégories d'événements de signal :

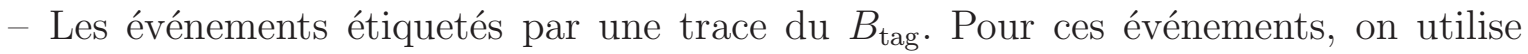
réellement la distance entre les vertex des deux $B$ pour déterminer $\Delta t$ et on peut mesurer la violation de $C P$.

- Les événements étiquetés par une trace du $D^{0}$ non reconstruit. Du fait de la méthode de reconstruction partielle utilisée, il est possible que l'on utilise une trace du $D^{0}$ non reconstruit, et provenant donc de $B_{\text {rec }}$, pour déterminer la saveur de $B_{\text {tag }}$. Cette trace est également utilisée pour ajuster la position du vertex de $B_{\text {tag }}$. Ainsi, le $\Delta z$ mesuré ne correspond pas à la vraie distance entre les vertex des deux $B$, et on ne peut pas mesurer la violation de $C P$ dépendante du temps avec ce type d'événements.

On tient compte de ces deux types d'événements dans la paramétrisation de la PDF en $\Delta t$. Dans la partie 5.5.2 sera exposé le traitement des événements étiquetés par une trace du $B_{\text {tag }}$, puis on expliquera dans la partie 5.5.4 le cas des événements étiquetés par un trace du $D^{0}$ non reconstruit.

\subsubsection{PDF en $\Delta t$ du signal $D^{*} \pi$ pour les événements étiquetés par une trace issue de $B_{\text {tag }}$}

\section{$\mathrm{PDF}$ en $\Delta t$}

En l'absence de tout effet expérimental, la PDF $\mathcal{T}_{\text {ideal }}$ du signal est donnée par les équations de la partie 4.2.2. Ces équations nous donnent les amplitudes de transition $\left\langle D^{*-} \pi^{+}|\mathcal{T}| B^{0}\right\rangle,\left\langle D^{*-} \pi^{+}|\mathcal{T}| \bar{B}^{0}\right\rangle,\left\langle D^{*+} \pi^{-}|\mathcal{T}| B^{0}\right\rangle$ et $\left\langle D^{*+} \pi^{-}|\mathcal{T}| \bar{B}^{0}\right\rangle$. Il est possible de réécrire ces quatre équations en une seule qui dépend des variables $S_{\text {tag }}$ et $S_{\text {mix }}$, définies dans la partie 5.3. Il s'agit de déterminer quelles sont les valeurs de $S_{\text {tag }}$ et de $S_{\text {mix }}$ pour chacune de ces désintégrations. Considérons l'amplitude $\left\langle D^{*-} \pi^{+}|\mathcal{T}| B^{0}\right\rangle$ : on a un pion dur de charge positive qui provient d'un $B^{0}$. Cela correspond à la transition favorisée du $B^{0}$ en $D^{*} \pi$ et on considère que cet événement ne s'est pas "mélangé" $\left(S_{\text {mix }}=1\right)$. On considère donc que les deux $B$ ont la même saveur au moment de leur désintégration. Ici, $B_{\text {rec }}$ est un $B^{0}$ et par conséquent $B_{\text {tag }}$ est un $\bar{B}^{0}\left(S_{\text {tag }}=-1\right)$. Par des raisonnements analogues, on peut déduire les valeurs de $S_{\text {tag }}$ et de $S_{\text {mix }}$ pour toutes les amplitudes. Ces valeurs sont résumées dans le tableau 5.7. 


\begin{tabular}{|c|r|r|}
\cline { 2 - 3 } \multicolumn{1}{c|}{} & $S_{\text {tag }}$ & $S_{\text {mix }}$ \\
\hline$\left\langle D^{*-} \pi^{+}|\mathcal{T}| B^{0}\right\rangle$ & -1 & 1 \\
$\left\langle D^{*-} \pi^{+}|\mathcal{T}| \bar{B}^{0}\right\rangle$ & 1 & -1 \\
$\left\langle D^{*+} \pi^{-}|\mathcal{T}| B^{0}\right\rangle$ & -1 & -1 \\
$\left\langle D^{*+} \pi^{-}|\mathcal{T}| \bar{B}^{0}\right\rangle$ & 1 & 1 \\
\hline
\end{tabular}

TAB. 5.7: Valeurs de $S_{\text {tag }}$ et $S_{\text {mix }}$ pour les différents types d'amplitudes

En fonction des variables $S_{\text {mix }}$ et $S_{\text {tag }}$, la distribution de $\Delta t$ vrai (par opposition au $\Delta t$ mesuré) est donné par :

$$
\begin{aligned}
\mathcal{T}_{\text {ideal }}\left(\Delta t_{\text {vrai }}, S_{\text {mix }}, S_{\text {tag }}\right)= & \frac{1}{8 \tau} \exp \left(-\frac{\left|\Delta t_{\text {vrai }}\right|}{\tau}\right) \\
& {\left[1+S_{\text {mix }} A \cos \left(\Delta m \Delta t_{\text {vrai }}\right)\right.} \\
& \left.-\left(S_{\text {tag }} a+S_{\text {tag }} S_{\text {mix }} b-S_{\text {mix }} c\right) \sin \left(\Delta m \Delta t_{\text {vrai }}\right)\right]
\end{aligned}
$$

où $\Delta m$ représente la fréquence d'oscillation $B^{0} / \bar{B}^{0}$ et $\tau$ le temps de vie du $B^{0}$.

Cette PDF est représentée figure 5.15 sans violation de $C P(a=b=c=0)$ et figure 5.16 avec violation de $C P(a=0,08$ et $b=c=0)$. La violation de $C P$ a été doublée par rapport à la valeur attendue, afin de mieux mettre l'effet en évidence. On remarque que l'effet de la violation de $C P$ affecte principalement la PDF des événements "mélangés". Notons que ces PDF ne tiennent pas compte de la résolution.

La PDF "idéale" exposée ci-dessus ne rend pas réellement compte de la réalité. En effet, il faut tenir compte des effets dûs au mauvais étiquetage. Ces effets modifient l'expression de la PDF.

Étudions les effets du mauvais étiquetage sur la PDF. Soit $\omega_{1}\left(\omega_{2}\right)$ la fraction d'événements dont le $B_{\text {tag }}$ est identifié comme étant un $B^{0}\left(\bar{B}^{0}\right)$. Considérons les événements reconstruits étiquetés par un $B^{0}\left(S_{\mathrm{tag}}=-1\right)$ et avec $S_{\text {mix }}=1$. Parmi les événements tels que $S_{\text {mix }}=1$ et $S_{\text {tag }}=-1$, une fraction $\left(1-\omega_{1}\right)$ a été bien étiquetée et contribue aux événements considérés. D'autre part, parmi les événements tels que $S_{\mathrm{mix}}=-1$ et $S_{\mathrm{tag}}=1$, une fraction $\omega_{2}$ a été mal étiquetée et correspond également à ces événements. Si on tient compte du mauvais étiquetage, la PDF, que l'on note alors $\mathcal{T}_{\omega}$, s'écrit alors :

$$
\begin{aligned}
\mathcal{T}_{\omega}\left(\Delta t_{\text {vrai }}, S_{\text {mix }}=1, S_{\text {tag }}=-1\right)= & \left(1-\omega_{1}\right) \mathcal{T}_{\text {ideal }}\left(\Delta t_{\text {vrai }}, S_{\text {mix }}=1, S_{\text {tag }}=-1\right) \\
& +\omega_{2} \mathcal{T}_{\text {ideal }}\left(\Delta t_{\text {vrai }}, S_{\text {mix }}=-1, S_{\text {tag }}=1\right) \\
= & \frac{1}{8 \tau} \exp \left(-\frac{\left|\Delta t_{\text {vrai }}\right|}{\tau}\right) \\
& {\left[(1-\Delta \omega)+(1-2 w) A \cos \left(\Delta m \Delta t_{\text {vrai }}\right)\right.} \\
& \left.+((1-2 \omega)(a-c)+b(1+\Delta \omega)) \sin \left(\Delta m \Delta t_{\text {vrai }}\right)\right], \\
&
\end{aligned}
$$

où $\Delta \omega=\omega_{1}-\omega_{2}$ et $\omega=\frac{\omega_{1}+\omega_{2}}{2}$. 

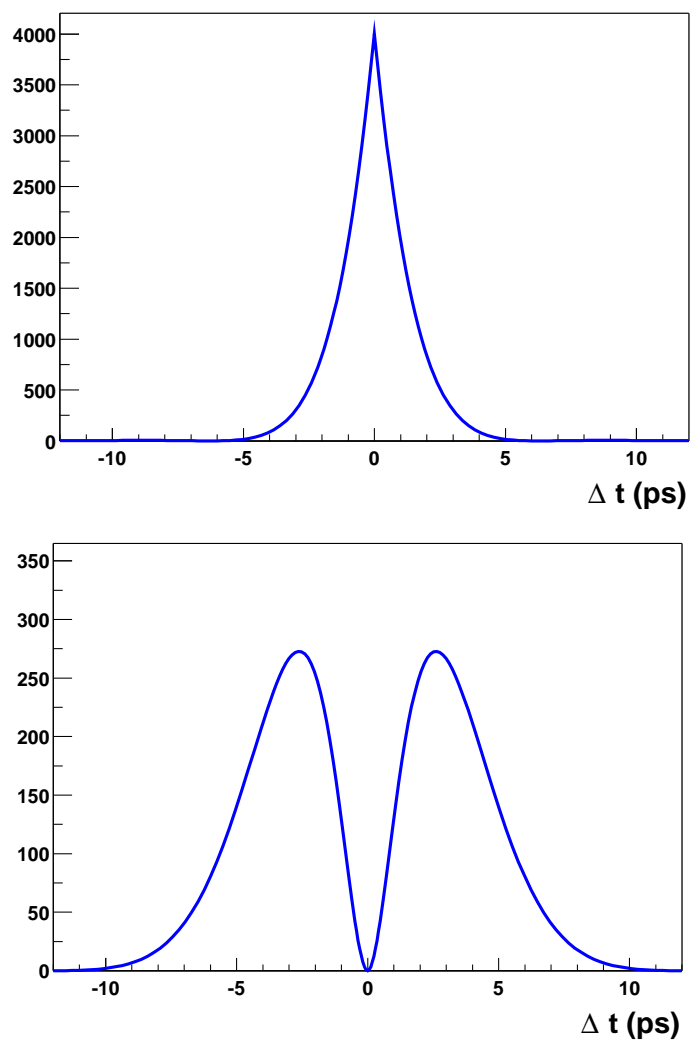

FIG. 5.15: PDF $\mathcal{T}_{\text {ideal }}$ pour les événements "non mélangés" (haut) et "mélangés" (bas), sans violation de $C P$.

La différence d'efficacité de détection entre des particules identiques mais de charge opposée, qui est due au fait que le détecteur est constitué de matière, a pour conséquence que l'on n'a pas forcément le même nombre d'événements étiquetés par un $B^{0}$ ou un $\bar{B}^{0}$. Afin de tenir compte de cela, on rajoute une variable $\epsilon$, telle que le nombre d'événements étiquetés par un $B^{0}$ soit $N(1+\epsilon)$ et le nombre d'événements étiquetés par un $\bar{B}^{0}$ soit $N(1-\epsilon)$.

De manière générale, la PDF s'écrit en tenant compte du mauvais étiquetage :

$$
\begin{aligned}
\mathcal{T}_{\omega}\left(\Delta t_{\text {vrai }}, S_{\mathrm{mix}}, S_{\mathrm{tag}}\right)= & \left(1-S_{\mathrm{tag}} \epsilon\right) \frac{1}{8 \tau} \exp \left(-\frac{\left|\Delta t_{\text {vrai }}\right|}{\tau}\right) \\
& {\left[\left(1-S_{\mathrm{tag}} \Delta \omega\right)+S_{\mathrm{mix}}(1-2 \omega) A \cos \left(\Delta m \Delta t_{\text {vrai }}\right)\right.} \\
& \left.-\left((1-2 \omega)\left(S_{\mathrm{tag}} a+S_{\mathrm{mix}} c\right)+S_{\mathrm{tag}} S_{\mathrm{mix}} b\left(1-S_{\mathrm{tag}} \Delta \omega\right)\right) \sin \left(\Delta m \Delta t_{\text {vrai }}\right)\right] .
\end{aligned}
$$

Cette PDF est ensuite convoluée avec une fonction de résolution qui est décrite au paragraphe suivant. 

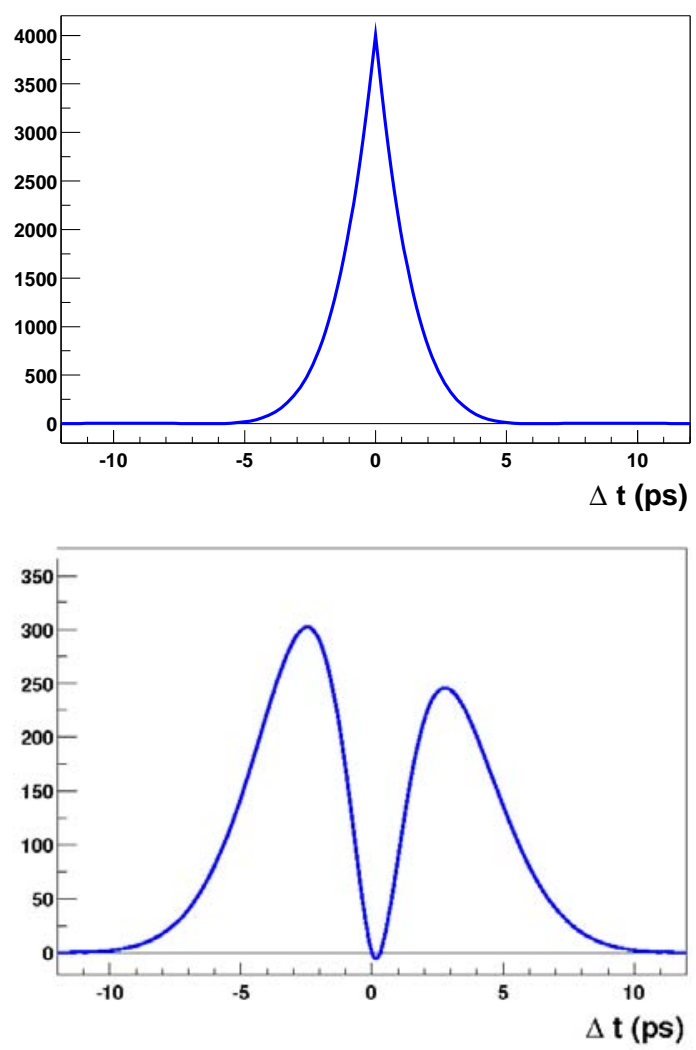

FIG. 5.16: PDF $\mathcal{T}_{\text {ideal }}$ pour les événements "non mélangés" (haut) et "mélangés" (bas), avec violation de $C P$.

\section{La fonction de résolution temporelle}

On calcule $\Delta t$ de la façon exposée dans la partie 5.1.5. Si l'erreur sur $\Delta t$ était parfaitement connue, la fonction de résolution serait une gaussienne de largeur 1, normalisée par l'erreur estimée pour chaque événement. Cependant, il faut tenir compte de certains effets systématiques qui compliquent cette modélisation :

1. $\sigma_{\Delta t}$ est de manière générale sous estimée en raison du mauvais alignement éventuel du détecteur de vertex. Pour modéliser la résolution réelle en $\Delta t$, il faut donc élargir la résolution obtenue à partir de l'erreur calculée événement par événement.

2. Il faut également tenir compte du vol du charme produit par la désintégration du $B_{\text {tag }}$ dans la direction du boost. Les particules secondaires issues des mésons charmés et utilisées dans l'ajustement du vertex ont tendance à biaiser la position du vertex dans le sens des z positifs et à dégrader la résolution du vertex. Afin de modéliser cet effet, on ajoute une deuxième gaussienne large (par opposition à la première que l'on qualifie d'étroite). Cette gaussienne possède un biais négatif.

3. On utilise également une troisième gaussienne, appelée "outlier", centrée en 0 et très large. Cette gaussienne permet de tenir compte de toutes les autres traces mal reconstruites ainsi que les traces provenant de la désintégration du $D^{0}$ non reconstruit, qui pourraient perturber la mesure du vertex. La contribution de cette 
gaussienne est généralement très faible.

On utilise donc une somme de trois gaussiennes pour modéliser la fonction de résolution :

$$
\mathcal{R}\left(t_{r}, \sigma_{\Delta t}\right)=f_{e} \mathcal{G}_{e}\left(t_{r}, \sigma_{\Delta t}\right)+\left(1-f_{e}-f_{o}\right) \mathcal{G}_{l}\left(t_{r}, \sigma_{\Delta t}\right)+f_{o} \mathcal{G}_{o}\left(t_{r}, \sigma_{\Delta t}\right)
$$

où $\mathcal{G}_{n}, \mathcal{G}_{w}$ et $\mathcal{G}_{o}$ sont les gaussiennes étroite, large et outlier. $f^{n}$ représente la fraction de $\mathcal{G}_{e}$ et $f^{o}$ la fraction de $\mathcal{G}_{o}$. On note $t_{r}$ le résidu entre $\Delta t$ mesuré et $\Delta t_{\text {vrai }}: t_{r}=\Delta t-\Delta t_{\text {vrai }}$.

Les gaussiennes étroite et large sont normalisées par rapport à $\sigma_{\Delta t}$ :

$$
\mathcal{G}_{n, w}\left(t_{r}, \sigma_{\Delta t}\right)=\frac{1}{\sqrt{2 \pi} s^{n, w} \sigma_{\Delta t}} \exp \left(-\frac{\left(t_{r}-b^{n, w} \sigma_{\Delta t}\right)^{2}}{2\left(s^{n, w} \sigma_{\Delta t}\right)^{2}}\right)
$$

Les paramètres $b^{n, w}$ et $s^{n, w}$ représentent les biais et largeurs des deux gaussiennes.

L'outlier ne dépend pas de $\sigma_{\Delta t}$ et possède la forme suivante :

$$
\mathcal{G}_{o}\left(t_{r}\right)=\frac{1}{\sqrt{2 \pi} s^{o}} \exp \left(-\frac{\left(t_{r}-b^{o}\right)^{2}}{2\left(s^{o}\right)^{2}}\right) .
$$

Le biais $b^{o}$ est fixé à 0 et la largeur $s^{o}$ est fixée à 8 ps.

\subsubsection{Effet des leptons de cascade sur la paramétrisation de la PDF}

On distingue deux types de leptons provenant de la désintégration du $B_{\text {tag }}$ et servant à étiqueter la saveur de celui-ci :

1. Les événements étiquetés par un lepton direct. On appelle lepton direct un lepton qui provient directement de la désintégration de $B_{\text {tag }}$, et dont la charge est entière-

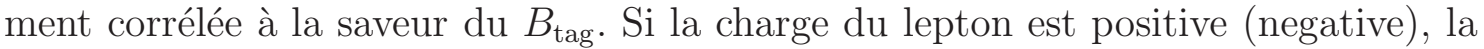
saveur du $B_{\text {tag }}$ est $B^{0}\left(\bar{B}^{0}\right)$. La fraction de mauvais étiquetage pour ce type d'événements est donc quasiment nulle. De plus, le vertex n'est pas biaisé et on s'attend à ce que la fonction de résolution temporelle soit étroite et non biaisée pour ce type d'événements.

2. Les événements étiquetés par un lepton de cascade. On appelle lepton de cascade un lepton issu du $B_{\text {tag }}$, mais pas de façon directe. Environ $12 \%$ des événements sont étiquetés par un lepton de cascade. Ce type de lepton provient principalement de la désintégration du charme produit par le $B_{\text {tag. }}$. Dans ce cas-là, un $B^{0}\left(\bar{B}^{0}\right)$ peut produire un lepton de charge négative (positive), qui devient anti-corrélée à la saveur de $B_{\text {tag. }}$ La fraction de mauvais étiquetage $\omega$ est donc très grande (de l'ordre de $65 \%$ sur Monte-Carlo). Notons que cette fraction élevée de mauvais étiquetage ne signifie pas que l'on n'a aucune information sur l'étiquetage : elle signifie uniquement que

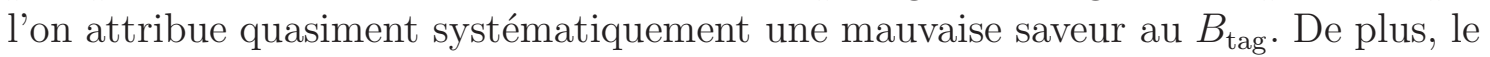
vol du charme a tendance à élargir la résolution sur la position du vertex du $B_{\text {tag }}$, avec un biais systématique dans le sens $z>0$, quel que soit le signe de $\Delta t$, comme l'illustre la figure 5.17. 


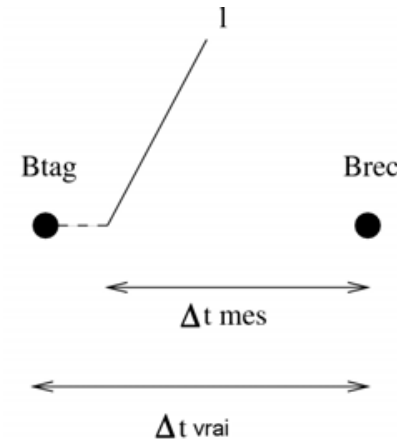

$\Delta \mathrm{t}>0: \Delta \mathrm{t}$ mes $<\Delta \mathrm{t}$ vrai

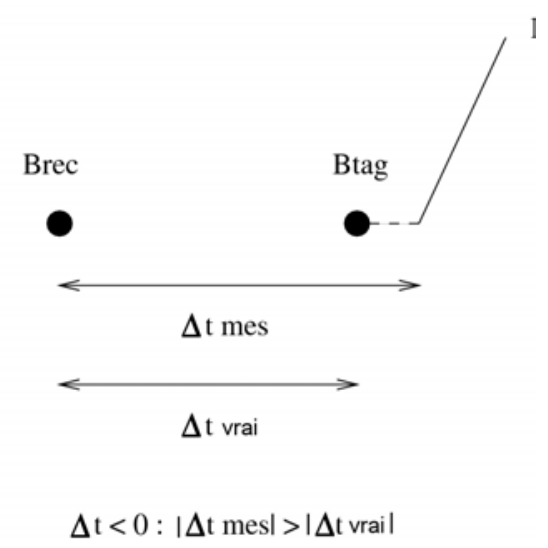

\section{$\Delta \mathrm{t}$ mes $<\Delta \mathrm{t}$ vrai}

Fig. 5.17: Effet des leptons de cascade sur la mesure de $\Delta t$ pour $\Delta t>0$ (gauche) et $\Delta t<0$ (droite). On peut remarquer que dans les deux cas, on mesure un $\Delta t$ plus petit que la réalité.

La fonction de résolution correspondant à ce type d'événements est donc plus large que pour les événements étiquetés par des leptons directs, et a un biais négatif.

Il existe un effet de corrélation entre les fractions de mauvais étiquetage et les fonctions de résolution de ces deux types d'événements, ce qui a pour conséquence de biaiser les paramètres de $C P$ mesurés.

La PDF en $\Delta t \mathcal{T}_{\text {dir }}^{\prime}$ pour les événements étiquetés par un lepton direct est :

$$
\mathcal{T}_{\text {dir }}^{\prime}=\mathcal{T}_{\text {dir }}\left(\Delta t_{\text {vrai }}, S_{\text {mix }}, S_{\text {tag }}\right) \otimes \mathcal{R}_{\text {dir }}\left(\Delta t_{\text {vrai }}, \sigma_{\Delta t}\right),
$$

et la PDF en $\Delta t \mathcal{T}_{\text {cas }}^{\prime}$ pour les événements étiquetés par un lepton de cascade est :

$$
\mathcal{T}_{\text {cas }}^{\prime}=\mathcal{T}_{\text {cas }}\left(\Delta t_{\text {vrai }}, S_{\text {mix }}, S_{\text {tag }}\right) \otimes \mathcal{R}_{\text {cas }}\left(\Delta t_{\text {vrai }}, \sigma_{\Delta t}\right) .
$$

La PDF totale qui décrit ces deux types d'événements est donc la somme de ces deux PDFs pondérée par la fraction d'événements étiquetés par un lepton de cascade (notée $\left.f_{\text {cas }}\right)$ :

$$
\begin{aligned}
\mathcal{T}_{\text {dir }+ \text { cas }}^{\prime}= & \left(1-f_{\text {cas }}\right) \mathcal{T}_{\text {dir }}\left(\Delta t_{\text {vrai }}, S_{\text {mix }}, S_{\text {tag }}\right) \otimes \mathcal{R}_{\text {dir }}\left(\Delta t_{\text {vrai }}, \sigma_{\Delta t}\right) \\
& +f_{\text {cas }} \mathcal{T}_{\text {cas }}\left(\Delta t_{\text {vrai }}, S_{\text {mix }}, S_{\text {tag }}\right) \otimes \mathcal{R}_{\text {cas }}\left(\Delta t_{\text {vrai }}, \sigma_{\Delta t}\right)
\end{aligned}
$$

Les PDFs $\mathcal{T}_{\text {dir }}$ et $\mathcal{T}_{\text {cas }}$ dépendent des paramètres $\tau, \Delta m$, $\omega$ et des paramètres de $C P a$, $b$ et $c . \tau, \Delta m$ et les paramètres de $C P$ ont la même signification physique pour les deux types d'événements. Le seul paramètre qui diffère est la fraction de mauvais étiquetage 
$\omega$, pour les raisons qui sont évoquées plus haut. Les fonctions de résolution $\mathcal{R}_{\text {dir }}$ et $\mathcal{R}_{\text {cas }}$ sont également différentes.

Supposons que les événements étiquetés par un lepton direct ou de cascade aient la même fraction de mauvais étiquetage. Dans ce cas-là, les PDFs $\mathcal{T}_{\text {dir }}$ et $\mathcal{T}_{\text {cas }}$ sont les mêmes. Par linéarité de la convolution, on peut donc factoriser l'expression 5.29 de la façon suivante :

$$
\begin{aligned}
\mathcal{T}_{\text {dir }+ \text { cas }}^{\prime}= & \mathcal{T}\left(\Delta t_{\text {vrai }}, S_{\text {mix }}, S_{\text {tag }}\right) \otimes \\
& {\left[\left(1-f_{\text {cas }}\right) \mathcal{R}_{\text {dir }}\left(\Delta t_{\text {vrai }}, \sigma_{\Delta t}\right)+f_{\text {cas }} \mathcal{R}_{\text {cas }}\left(\Delta t_{\text {vrai }}, \sigma_{\Delta t}\right)\right] . }
\end{aligned}
$$

On peut alors décrire ces deux types d'événements par une seule PDF $\mathcal{T}$ convoluée avec une fonction de résolution moyennée sur les deux types d'événements.

De la même façon, si ces deux types d'événements ont la même fonction de résolution, on peut factoriser l'expression 5.29 de la façon suivante :

$$
\mathcal{T}_{\text {dir }+ \text { cas }}^{\prime}=\mathcal{T}\left(\Delta t_{\text {vrai }}, S_{\text {mix }}, S_{\mathrm{tag}}\right) \otimes \mathcal{R}\left(\Delta t_{\text {vrai }}, \sigma_{\Delta t}\right)
$$

avec :

$$
\begin{aligned}
\mathcal{T}= & \left(1-f_{\text {cas }}\right) \mathcal{T}_{\text {dir }}\left(\Delta t_{\text {vrai }}, S_{\text {mix }}, S_{\mathrm{tag}}\right)+f_{\text {cas }} \mathcal{T}_{\text {cas }}\left(\Delta t_{\text {vrai }}, S_{\text {mix }}, S_{\mathrm{tag}}\right) \\
= & \frac{1}{8 \tau} \exp \left(-\frac{\left|\Delta t_{\text {vrai }}\right|}{\tau}\right)\left[\left(1-S_{\mathrm{tag}} \Delta \omega\right)+S_{\mathrm{mix}}\left(1-2 \omega_{\text {eff }}\right) A \cos \left(\Delta m \Delta t_{\text {vrai }}\right)\right. \\
& \left.-\left(\left(1-2 \omega_{\text {eff }}\right)\left(S_{\mathrm{tag}} a+S_{\mathrm{mix}} c\right)+S_{\mathrm{tag}} S_{\mathrm{mix}} b\left(1-S_{\mathrm{tag}} \Delta \omega\right)\right) \sin \left(\Delta m \Delta t_{\text {vrai }}\right)\right]
\end{aligned}
$$

où $\omega_{\text {eff }}$ est la fraction de mauvaise étiquetage moyenne $: \omega_{\text {eff }}=\left(1-f_{\text {cas }}\right) \omega_{\text {dir }}+f_{\text {cas }} \omega_{\text {cas }}$. On peut là encore n'utiliser qu'une seule PDF pour les événements étiquetés par un lepton direct ou de cascade. Cependant, dans le cas où à la fois la fraction de mauvais étiquetage et la fonction de résolution sont différentes, il n'est pas possible de factoriser l'expression 5.29. Le fait de n'utiliser qu'une seule PDF pour ces deux types d'événements n'est donc pas équivalent à utiliser une PDF pour chacun de ces types d'événements.

La distribution de la résolution en $\Delta t$ peut être obtenue avec des événements simulés de signal par :

$$
\frac{\Delta t_{m e s}-\Delta t_{v r a i}}{\sigma_{\Delta t}}
$$

Les distributions correspondantes pour les événements simulés de signal étiquetés par un lepton direct ou de cascade sont représentées figure 5.18.

Les fonctions de résolution de ces deux types d'événements sont différentes. D'autre part, on a vu plus tôt que les fractions de mauvais étiquetage étaient également différentes. Pour les raisons exposées plus haut, il est donc nécessaire d'utiliser une PDF pour chacun de ces types d'événements de signal étiqueté par un lepton, afin d'éviter de biaiser la mesure des paramètres de $C P$. Une première version de cette analyse n'utilisait qu'une seule fonction de résolution pour ces deux types d'événements. Cela se traduisait par un 

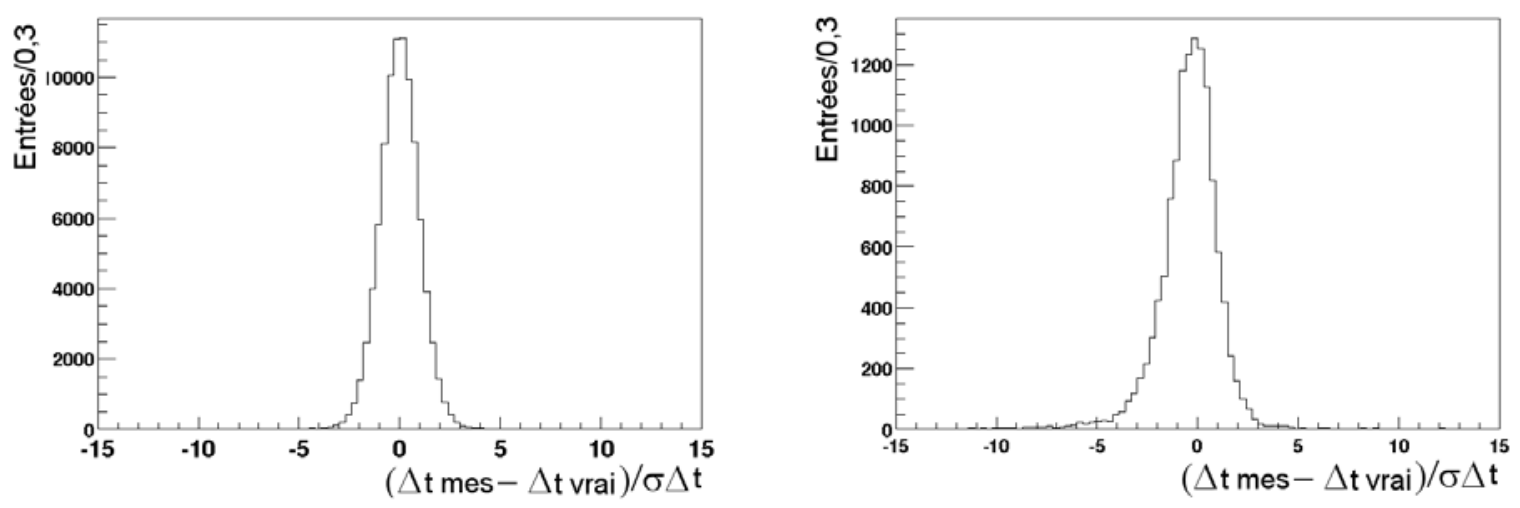

FIG. 5.18: Distribution de la fonction de résolution des événements simulés de signal étiquetés par un lepton direct (gauche) et de cascade (droite).

biais sur la mesure des paramètres de violation de $C P$ que nous avons d'abord évalué. Ensuite, nous nous sommes attachés à améliorer l'analyse afin d'éliminer ce biais.

On peut comprendre intuitivement le signe des biais observés par une simple analyse de l'effet. L'effet est particulièrement important pour les événements "mélangés". En effet, si on suppose que l'étiquetage est parfait, la plupart des événements sont en réalité "non mélangés". La fraction de mauvais étiquetage des événements étiquetés par un lepton direct étant très faible, la plupart de ces événements sont effectivement considérés comme étant "non mélangés". En revanche, la fraction de mauvais étiquetage des événements étiquetés par un lepton de cascade est très grande (de l'ordre de $65 \%$ ). Par conséquent, une grande partie de ces événements sont considérés à tort comme étant "mélangés" dans l'analyse. Ainsi, la fraction d'événements étiquetés par un lepton de cascade est plus importante pour les événements "mélangés" que pour les événements "non mélangés".

Soit $N$ le nombre d'événements total, $f_{\text {cas }}$ la fraction d'événements étiquetés par un lepton de cascade, $\chi$ la fraction réelle d'événements "mélangés" et $\omega_{\text {dir }}\left(\omega_{\text {cas }}\right)$ la fraction de mauvais étiquetage. Le nombre d'événements "mélangés" pour un lepton direct (de cascade $)$ est $\left(1-f_{\text {cas }}\right)\left(\chi+\omega_{\text {dir }}\right) N\left(f_{\text {cas }}\left(\chi+\omega_{\text {cas }}\right) N\right)$. Avec $f_{\text {cas }}=0,12, \chi=0,18, \omega_{\text {dir }} \sim 0$ et $\omega_{\text {cas }}=0,65$, on a donc $0,16 N(0,10 N)$ événements "mélangés" pour les événements étiquetés par un lepton direct (de cascade). Parmi les événements "mélangés", 38 \% sont donc étiquetés par un lepton de cascade. Pour mieux comprendre l'impact que cela peut avoir sur la mesure des paramètres de $C P$, on a généré des événements "mélangés", en respectant les proportions, avec les caractéristiques suivantes :

- $62 \%$ d'événements avec une fraction de mauvais étiquetage nulle et une fonction de résolution centrée en 0 , pour simuler les événements étiquetés par un lepton direct,

- $38 \%$ d'événements avec une fraction de mauvais étiquetage égale à $65 \%$ et une fonction de résolution centrée en -2 ps, pour simuler les événements étiquetés par un lepton de cascade.

La figure 5.19 montre les distributions en $\Delta t$ de ces deux catégories, ainsi que la somme de ces deux distributions. 

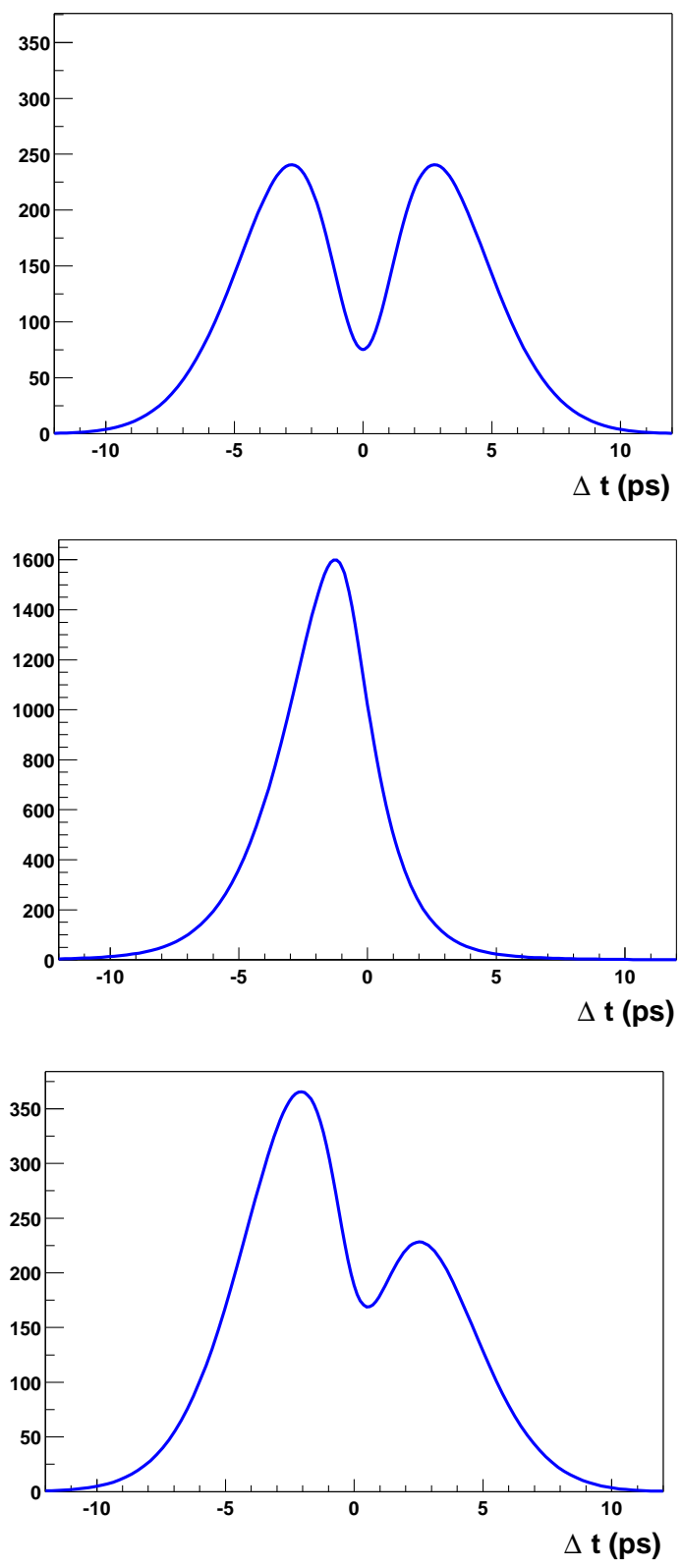

FIG. 5.19: PDF pour des événements "mélangés" étiquetés par un lepton direct avec $\omega_{D^{*} \pi}=0$ et une fonction de résolution centrée en 0 (haut) ; PDF pour des événements "mélangés" étiquetés par un lepton de cascade avec $\omega_{D^{*} \pi}=0,65$ et une fonction de résolution centrée en -2 ps (milieu); Somme de ces deux PDF, avec les mêmes fractions que dans le Monte-Carlo (bas).

On voit clairement sur cette figure que la distribution de la somme des deux types d'événements présente une asymétrie entre $\Delta t$ et $-\Delta t$, qui "simule" un effet de violation de $C P$ similaire à celui présenté par la figure 5.16. Si cet effet n'est pas bien pris en compte dans la paramétrisation de la $\mathrm{PDF}$ en $\Delta t$, il en résulte un biais sur les paramètres de $C P$.

Nous avons utilisé la paramétrisation initiale de l'évolution temporelle, qui dépend de $\sin (2 \beta+\gamma+\delta)$ et de $\sin (2 \beta+\gamma-\delta)$ (voir équations 4.19 à 4.21), afin de quantifier le biais. 
Dans le cas des événements étiquetés par un lepton, cette paramétrisation est totalement analogue à la paramétrisation $a b c$ (voir équations 4.23 à 4.25) utilisée dans l'analyse. Un échantillon d'événements simulés de signal a été utilisé pour ajuster les PDFs des événements étiquetés par un lepton direct ou de cascade séparément. Puis ces PDFs ont été utilisées pour simuler rapidement de nombreuses pseudo-expériences, sans violation de $C P$. On génère séparément les événements étiquetés par un lepton direct ou de cascade. Ensuite, on ajuste l'ensemble des événements ainsi générés par une seule PDF en $\Delta t$ en faisant varier les paramètres $\sin (2 \beta+\gamma+\delta)_{D^{*} \pi}, \sin (2 \beta+\gamma+\delta)_{D^{*} \pi}, \tau_{D^{*} \pi}, \Delta m_{D^{*} \pi}$ et $\omega_{D^{*} \pi}$, ainsi que les paramètres de la fonction de résolution. On recommence cette procédure 1000 fois. La figure 5.20 représente la distribution des valeurs ajustées de $\sin (2 \beta+\gamma+\delta)_{D^{*} \pi}$ et $\sin (2 \beta+\gamma-\delta)_{D^{*} \pi}$, ainsi que leurs erreurs.
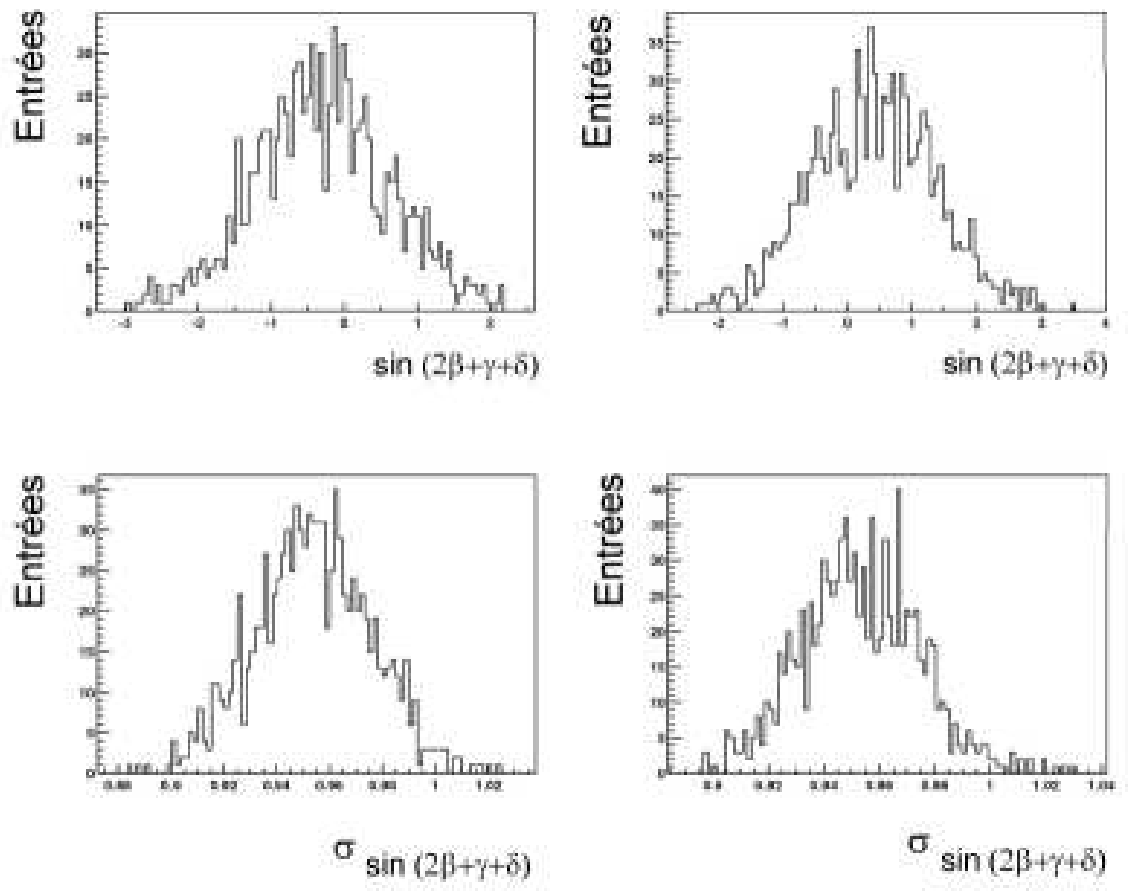

FIG. 5.20: Distribution des valeurs ajustées de $\sin (2 \beta+\gamma+\delta)_{D^{*} \pi}$ (en haut à gauche) et $\sin (2 \beta+$ $\gamma-\delta)_{D^{*} \pi}$ (en haut à droite), ainsi que leurs erreurs (en bas).

S'il n'y avait pas de biais, ces distributions devraient être centrées en 0, car on a généré des événements sans violation de $C P$. Or, on remarque que la distribution de $\sin (2 \beta+\gamma+\delta)_{D^{*} \pi}$ est centrée en $-0,3$ et que la distribution en $\sin (2 \beta+\gamma-\delta)_{D^{*} \pi}$ est centrée en 0,3 . Il y a donc un biais très clair sur la mesure des paramètres de $C P$ lorsque l'on n'utilise qu'une seule PDF pour décrire ces deux types d'événements de signal. Les caractéristiques du biais sur la mesure de $\sin (2 \beta+\gamma+\delta)_{D^{*} \pi}$ et $\sin (2 \beta+\gamma-\delta)_{D^{*} \pi}$ sont résumées dans le tableau 5.8.

Notons cependant que ce biais reste relativement faible. Lorsque l'on passe à la paramétrisation $a b c$, ce biais s'annule pour le paramètre $a_{D^{*} \pi}=r\left(\sin (2 \beta+\gamma+\delta)_{D^{*} \pi}+\right.$ 


\begin{tabular}{|l|c|}
\hline $\sin (2 \beta+\gamma+\delta)_{D^{*} \pi}$ & $-0,31 \pm 0,03$ \\
\hline $\sin (2 \beta+\gamma-\delta)_{D^{*} \pi}$ & $0,30 \pm 0,03$ \\
\hline
\end{tabular}

TAB. 5.8: Ajustement de $\sin (2 \beta+\gamma+\delta)_{D^{*} \pi}$ et $\sin (2 \beta+\gamma-\delta)_{D^{*} \pi}$ réalisé sur 1000 simulations rapides d'événements de signal, générées avec des PDFs différentes pour les événements étiquetés par un lepton direct ou de cascade. L'erreur sur le biais reflète le nombre de pseudo-expériences réalisées pour cette étude.

$\sin (2 \beta+\gamma-\delta)_{D^{*} \pi}$ mais est toujours présent dans le paramètre $c_{D^{*} \pi}=r(\sin (2 \beta+\gamma+$ $\delta)_{D^{*} \pi}-\sin (2 \beta+\gamma-\delta)_{D^{*} \pi}$.

On a ensuite testé la dépendance du biais observé en fonction de la vraie valeur des asymétries de $C P$. Pour cela, on a reproduit la même étude mais en générant cette foisci des événements avec violation de $C P$. La figure 5.21 montre l'amplitude du biais en fonction de la valeur générée pour le paramètre de $C P \sin (2 \beta+\gamma+\delta)$. Comme on s'y attendait, la dépendance de l'amplitude de l'effet en fonction de la vraie asymétrie de $C P$ est très faible.

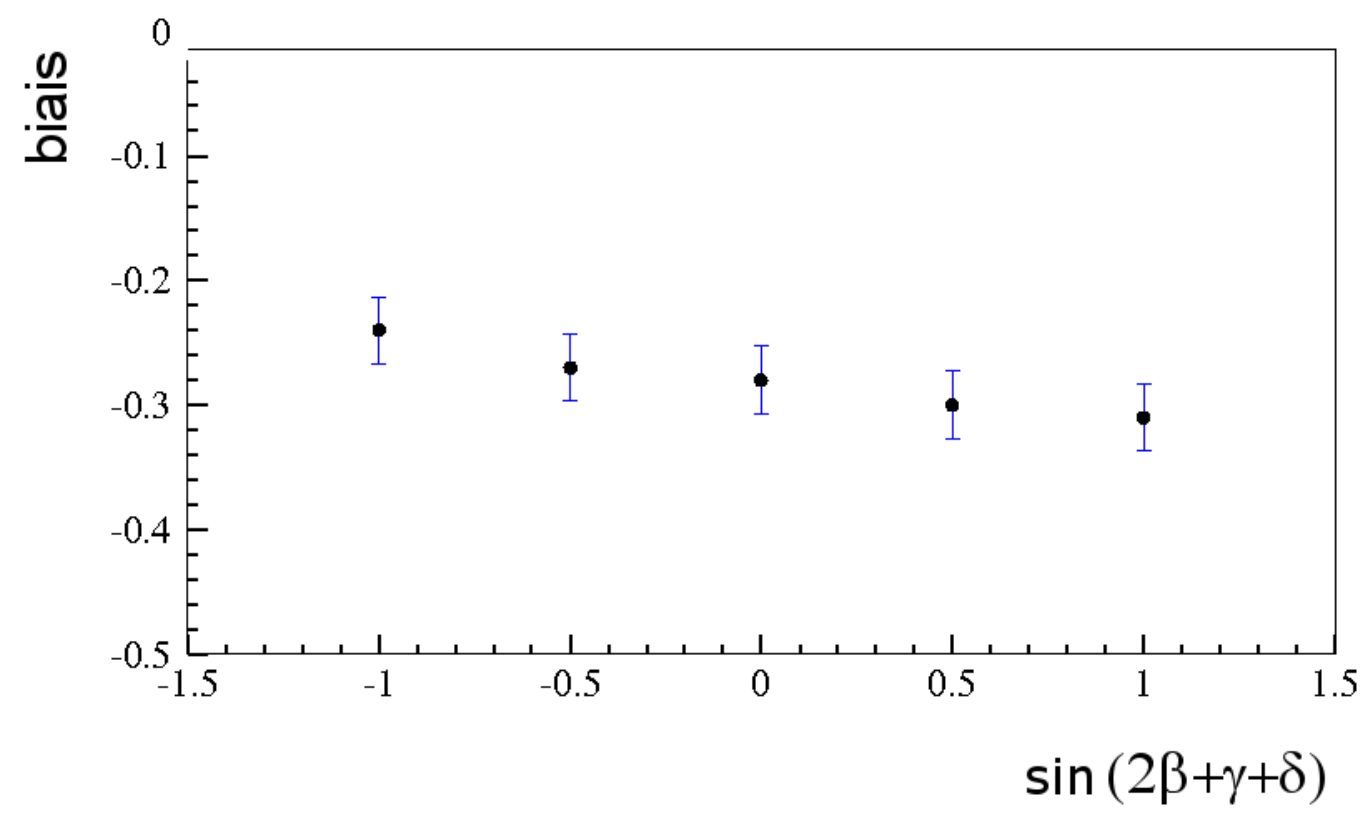

FIG. 5.21: Amplitude du biais sur l'ajustement de $\sin (2 \beta+\gamma+\delta)_{D^{*} \pi}$ en fonction de la valeur générée pour $\sin (2 \beta+\gamma+\delta)_{D^{*} \pi}$. Les erreurs sont dues au nombre fini de pseudoexpériences réalisées.

Afin de ne pas biaiser les paramètres de $C P$ mesurés, on utilise donc une PDF pour les événements étiquetés par un lepton direct et une PDF pour les événements étiquetés par un lepton de cascade. 
Pour les événements étiquetés par un kaon, on ne peut pas parler de kaons directs, car les kaons sont produits par la cascade $b \rightarrow c \rightarrow s$. On ne distingue pas de catégories de kaons provenant du $B_{\text {tag }}$ et on n'utilise donc qu'une seule PDF dans le cas des événements étiquetés par un kaon.

\subsubsection{Effet de la reconstruction partielle sur la paramétrisation de la PDF en $\Delta t$}

Il s'agit maintenant de prendre en compte l'effet de la reconstruction partielle sur la paramétrisation de la PDF. Jusqu'à présent, on n'a évoqué que le cas des événements étiquetés par une trace provenant du $B_{\mathrm{tag}}$, ce qui est la situation intéressante pour étudier la violation de $C P$ dépendante du temps. Il est toutefois possible que l'événement soit étiqueté à tort par une trace du $D^{0}$.

Pour les événements étiquetés par une trace issue du $D^{0}$ non reconstruit, la variable $\Delta z$ mesurée n'est pas la distance entre les vertex de $B_{\text {rec }}$ et de $B_{\text {tag }}$, mais la distance entre les vertex de $B_{\text {rec }}$ et du $D^{0}$. Le terme de mélange n'a pas lieu d'être pour ce type d'événements. La PDF qui décrit ces événements est donc une pure fonction exponentielle :

$$
\mathcal{T}_{\text {rp }}\left(\Delta t_{\text {vrai }}\right)=\exp \left(-\frac{\left|\Delta t_{\text {vrai }}\right|}{\tau^{\prime}}\right)
$$

Ici, $\tau^{\prime}$ représente un temps de vie effectif, qui correspond au premier ordre au temps de vie du $D^{0}\left(\tau_{D}^{0}=0,4103 \pm 0,0015\right.$ ps [29]).

Une fraction $(1-\alpha)$ des événements est décrite par la PDF $\mathcal{T}_{\omega}$. Pour les événements qualifiés de "mélangés" $\left(S_{\mathrm{mix}}=-1\right)$ une fraction $\alpha \rho$ est décrite par $\mathcal{T}_{r p}$ et pour les événements qualifiés de "non-mélangés", une fraction $\alpha(1-\rho)$ est décrite par $\mathcal{T}_{r p}$. Si on définit $\kappa=1-2 \rho$, on trouve l'expression générale de $\mathcal{T}^{\prime}$, qui prend en compte les effets expérimentaux :

$$
\mathcal{T}^{\prime}\left(\Delta t_{\text {vrai }}, S_{\text {mix }}, S_{\text {tag }}\right)=(1-\alpha) \mathcal{T}_{\omega}\left(\Delta t_{\text {vrai }}, S_{\text {mix }}, S_{\text {tag }}\right)+\alpha\left(1+S_{\text {mix }} \kappa\right) \mathcal{T}_{\text {rp }}\left(\Delta t_{\text {vrai }}\right)
$$

\subsubsection{Validation sur Monte-Carlo pour les événements étiquetés par un lepton}

Validation de la procédure avec des événements simulés de signal, générés sans violation de $C P$

La procédure de l'ajustement de la distribution en $\Delta t$ du signal a d'abord été testée sur du Monte-Carlo généré sans violation de $C P$. On utilise pour cette étude le signal provenant de l'échantillon d'événements simulés de signal et de l'échantillon de $B^{0}$ génériques. La luminosité équivalente totale est de $1353 \mathrm{fb}^{-1}$. Pour les événements étiquetés par un lepton, cela correspond à 102943 événements de signal. La répartition entre les différents types d'étiquetage est résumée dans le tableau 5.9. 


\begin{tabular}{|c|c|c|}
\hline type de lepton étiquetant & nombre de signal & fraction \\
\hline lepton direct & 89526 & $87 \%$ \\
lepton de cascade & 12496 & $12 \%$ \\
lepton provenant du $D^{0}$ & 925 & $1 \%$ \\
\hline
\end{tabular}

TAB. 5.9: Répartition du signal selon les différents types de lepton étiquetants

La distribution en $\Delta t$ des événements de signal $D^{*} \pi$ étiquetés par un lepton est modélisée par trois PDFs, selon le type de lepton étiquetant. Utiliser trois gaussiennes pour la fonction de résolution de chaque catégorie reviendrait à ajuster un très grand nombre de paramètres. Des études ont été menées sur Monte-Carlo afin de vérifier s'il était possible de simplifier la modélisation de la fonction de résolution, et ce, afin d'éviter d'avoir de trop importantes corrélations entre les paramètres à ajuster.

Pour les événements étiquetés par un lepton direct, il n'est pas forcément nécessaire d'utiliser la fonction de résolution classique à trois gaussiennes. En effet, on s'attend à ce que les contributions de la gaussienne large qui tient compte du vol du charme, principalement dans le $B_{\text {tag }}$ et de l'outlier qui modélise les événements mal reconstruits, soient négligeables pour ce type d'événements. Dans un premier temps, on ajuste directement les paramètres de la fonction de résolution sur la distribution :

$$
\frac{\Delta t_{m e s}-\Delta t_{v r a i}}{\sigma_{\Delta t}} .
$$

Différents types de fonctions de résolutions, à une, deux et trois gaussiennes, ont ainsi été testés sur Monte-Carlo. Les distributions et les résultats de l'ajustement sont montrés dans les tableaux 5.10, 5.11 et 5.12 et la figure 5.22 .

\section{1. une gaussienne :}

la distribution est ajustée par une gaussienne de biais $b_{D^{*} \pi}^{n}$ et de largeur $s_{D^{*} \pi}^{n}$.

\begin{tabular}{|l|l|}
\hline$b_{D^{*} \pi}^{n}$ & $0,002 \pm 0,003$ \\
\hline$s_{D^{*} \pi}^{n}$ & $0,973 \pm 0,003$ \\
\hline
\end{tabular}

TAB. 5.10: Résultats de l'ajustement par une gaussienne de la distribution $\left(\Delta t_{\text {mes }}-\Delta t_{\text {vrai }}\right) / \sigma_{\Delta t}$ pour des événements simulés de signal étiquetés par un lepton direct.

Bien que l'ajustement paraisse raisonnable sur la figure 5.22, le niveau de confiance de l'ajustement, obtenu connaissant le $\chi^{2}$ et le nombre de degrés de liberté, est quasiment nul. Cette paramétrisation simpliste de la fonction de résolution ne sera donc pas retenue.

\section{2. deux gaussiennes :}

la distribution est ajustée par deux gaussiennes de biais $b_{D^{*} \pi}^{n}, b_{D^{*} \pi}^{w}$ et de largeurs $s_{D^{*} \pi}^{n}, s_{D^{*} \pi}^{w}$. La fraction de première gaussienne est notée $f_{D^{*} \pi}^{n}$.

L'ajustement est de bonne qualité et a un niveau de confiance de $92 \%$. Cette paramétrisation de la fonction de résolution est donc bien adaptée. 


\begin{tabular}{|c|c|}
\hline$b_{D^{*} \pi}^{n}$ & $0,002 \pm 0,003$ \\
$b_{D^{*} \pi}^{w}$ & $-0,003 \pm 0,006$ \\
\hline$f_{D^{*} \pi}^{n}$ & $0,989 \pm 0,003$ \\
\hline$s_{D^{*} \pi}^{n}$ & $0,946 \pm 0,004$ \\
$s_{D^{* \pi}}^{w}$ & $2,42 \pm 0,18$ \\
\hline
\end{tabular}

TAB. 5.11: Résultats de l'ajustement par deux gaussiennes de la distribution $\left(\Delta t_{m e s}-\right.$ $\left.\Delta t_{\text {vrai }}\right) / \sigma_{\Delta t}$ pour des événements simulés de signal étiquetés par un lepton direct.

3. trois gaussiennes : la distribution est ajustée par deux gaussiennes de biais $b_{D^{*} \pi}^{n}$, $b_{D^{*} \pi}^{w}$ et de largeurs $s_{D^{*} \pi}^{n}, s_{D^{*} \pi}^{w}$ et par un outlier de biais 0 et de largeur 8 ps. La fraction de première gaussienne est notée $f_{D^{*} \pi}^{n}$ et la fraction d'outlier $f_{D^{*} \pi}^{o}$.

\begin{tabular}{|c|c|}
\hline$b_{D^{* \pi}}^{n}$ & $0,002 \pm 0,004$ \\
$b_{D^{*} \pi}^{w}$ & $0,003 \pm 0,003$ \\
\hline$f_{D^{*} \pi}^{n}$ & $0,93 \pm 0,02$ \\
$f_{D^{*} \pi}^{o}$ & $0,0006 \pm 0,00005$ \\
\hline$s_{D^{*} \pi}^{n}$ & $0,906 \pm 0,009$ \\
$s_{D^{*} \pi}^{w}$ & $1,48 \pm 0,07$ \\
\hline
\end{tabular}

TAB. 5.12: Résultats de l'ajustement par trois gaussiennes de la distribution $\left(\Delta t_{m e s}-\right.$ $\left.\Delta t_{\text {vrai }}\right) / \sigma_{\Delta t}$ pour des événements simulés de signal, étiquetés par un lepton direct.

L'ajustement est également de bonne qualité, et a un niveau de confiance de $90 \%$.

On remarque que les biais sont compatibles avec 0, comme on s'y attendait pour les événements étiquetés par un lepton direct. Les paramétrisations à deux et trois gaussiennes pour la fonction de résolution sont bien adaptées. On choisit la solution intermédiaire à deux gaussiennes pour l'ajustement de référence. Cela représente un compromis entre la simplicité et la possibilité de prendre en compte des événements éventuellement mal reconstruits dans les données. La contribution de la gaussienne étroite est la plus importante, mais ajouter une deuxième gaussienne permet d'apporter plus de flexibilité à l'ajustement et d'améliorer considérablement la qualité de l'ajustement.

Ces différentes fonctions de résolution ont également été testées sur l'ajustement de la distribution en $\Delta t$ des événements simulés de signal, étiquetés par un lepton direct. On ajuste alors également les paramètres $\Delta m_{D^{*} \pi}, \tau_{D^{*} \pi}$, la fraction de mauvais étiquetage $\omega_{D^{*} \pi}$ et les paramètres de $C P a_{D^{*} \pi}$ et $c_{D^{*} \pi}$.

Les résultats pour ces différents types de paramétrisation sont résumés dans le tableau 5.13 .

On peut remarquer que le choix de tel ou tel type de fonction de resolution n'influe pas l'ajustement des paramètres de $C P a_{D^{*} \pi}$ et $c_{D^{*} \pi}$. Ces derniers sont bien compatibles avec 0 . En revanche, le temps de vie $\tau_{D^{*} \pi}$, qui est très corrélé à la fonction de résolution, est légèrement biaisé lorsque l'on utilise plusieurs gaussiennes. En effet, on mesure $\tau_{D^{*} \pi}=$ $1,534 \pm 0,007 \mathrm{ps}$, alors que la valeur générée est $\tau_{D^{*} \pi}=1,548 \mathrm{ps}$. On observe donc un effet de deux déviations standard. Lorsque l'on utilise une fonction de résolution à une 

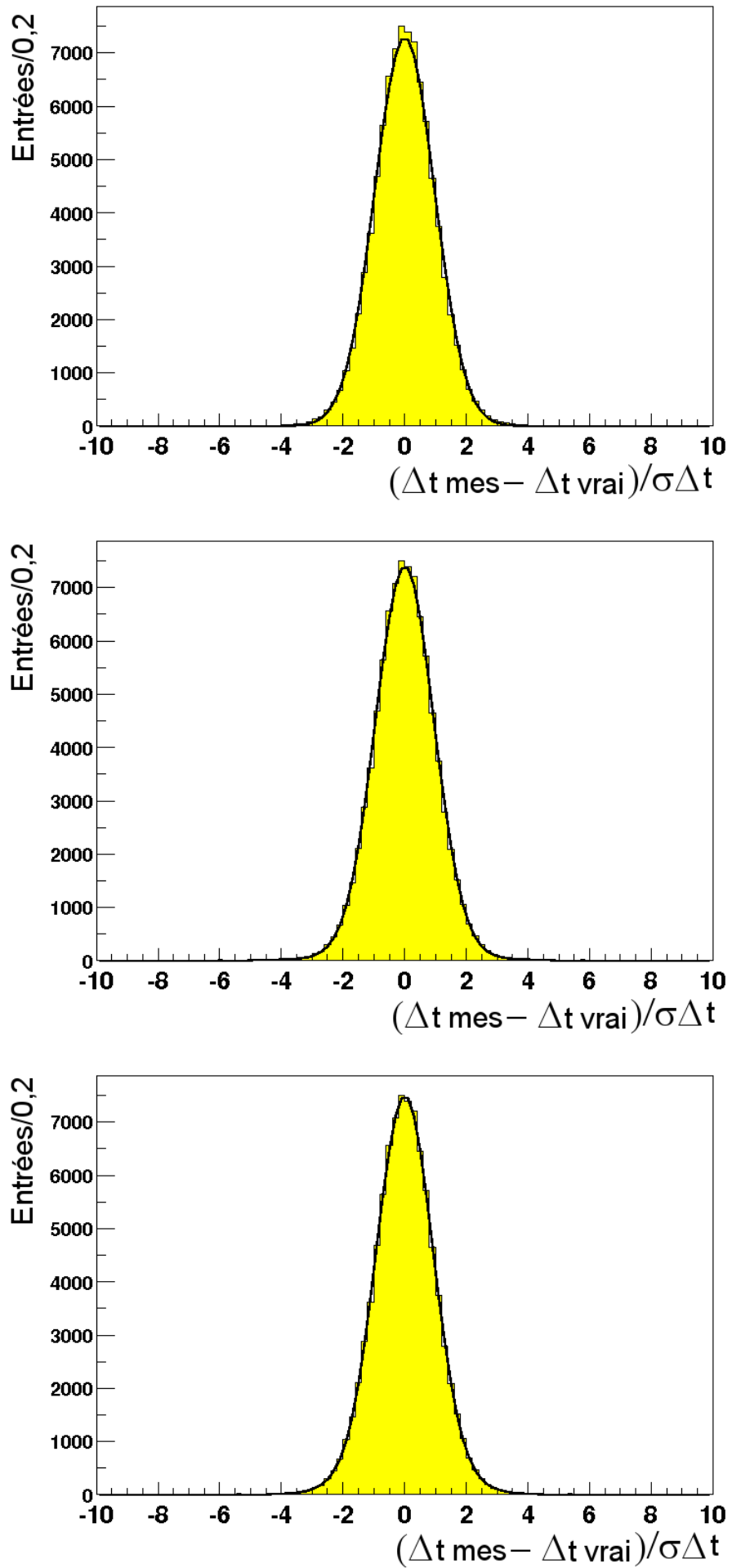

Fig. 5.22: Ajustement par une (haut), deux (milieu) ou trois (bas) gaussienne(s) de la distribution $\left(\Delta t_{\text {mes }}-\Delta t_{\text {vrai }}\right) / \sigma_{\Delta t}$ pour des événements simulés de signal, étiquetés par un lepton direct. 


\begin{tabular}{|c|c|c|c|c|}
\hline \multicolumn{5}{|c|}{ Signal, lepton direct } \\
\hline Paramètre & 3 gaussiennes & 2 gaussiennes & 1 gaussienne & valeur générée \\
\hline$a_{D^{*} \pi}$ & $0,002 \pm 0,005$ & $0,002 \pm 0,005$ & $0,001 \pm 0,005$ & 0 \\
\hline$c_{D^{*} \pi}$ & $-0,006 \pm 0,005$ & $-0,007 \pm 0,005$ & $-0,007 \pm 0,005$ & 0 \\
\hline$\Delta m_{D^{*} \pi}$ & $0,481 \pm 0,002$ & $0,482 \pm 0,002$ & $0,477 \pm 0,002$ & 0,489 \\
\hline$\tau_{D^{*} \pi}$ & $1,533 \pm 0,007$ & $1,534 \pm 0,007$ & $1,555 \pm 0,006$ & 1,548 \\
\hline$\omega_{D^{*} \pi}$ & $0,004 \pm 0,001$ & $0,004 \pm 0,001$ & $0,006 \pm 0,001$ & 0 \\
\hline$f_{D^{*} \pi}^{n}$ & $0,986 \pm 0,006$ & $0,980 \pm 0,005$ & 1 & \\
\hline$f_{D^{*} \pi}^{w}$ & $1-f_{D^{*} \pi}^{n}-f_{D^{*} \pi}^{o}$ & $1-f_{D^{*} \pi}^{n}$ & 0 & \\
\hline$f_{D^{*} \pi}^{o}$ & $0,006 \pm 0,001$ & 0 & 0 & \\
\hline$b_{D^{*} \pi}^{n}$ & $-0,024 \pm 0,007$ & $-0,010 \pm 0,008$ & $-0,007 \pm 0,008$ & \\
\hline$s_{D^{*} \pi}^{n}$ & $0,94 \pm 0,01$ & $0,931 \pm 0,013$ & $0,963 \pm 0,003$ & \\
\hline $\begin{array}{c}b_{D^{*} \pi}^{w} D^{*} \pi \\
s_{D^{*} \pi}^{w}\end{array}$ & $\begin{aligned} 3,0 & \pm 1,0 \\
1,22 & \pm 0,65\end{aligned}$ & $\begin{array}{l}0,29 \pm 0,25 \\
3,84 \pm 0,49\end{array}$ & & \\
\hline $\begin{array}{l}b_{D^{* \pi}}^{o} \\
S_{D^{*} \pi}^{o}\end{array}$ & $\begin{array}{l}0 \\
8\end{array}$ & & & \\
\hline
\end{tabular}

TAB. 5.13: Ajustement de la distribution en $\Delta t$ des événements simulés de signal, étiquetés par un lepton direct, avec différentes fonctions de résolution.

seule gaussienne, on mesure un $\tau_{D^{*} \pi}$ qui n'est qu'à une déviation standard de la valeur générée.

Dans l'analyse des données, on utilise une fonction de résolution à deux gaussiennes pour les leptons directs, ce qui biaise donc le temps de vie. On corrige alors $\tau_{D^{*} \pi}$ de la déviation observée sur Monte-Carlo lorsque l'on utilise plusieurs gaussiennes et on assigne une erreur systématique à $\tau_{D^{*} \pi}$. La correction appliquée est $\Delta \tau_{D^{*} \pi}=1,548-1,534=$ 0,014 ps, à laquelle on assigne une erreur systématique égale à la moitié de la correction, soit 0,007 ps.

La figure 5.23 montre le résultat de l'ajustement de la distribution en $\Delta t$ (avec une fonction de résolution à deux gaussiennes) pour les événements étiquetés par un lepton direct.

Des études similaires sur la fonction de résolution des événements étiquetés par un lepton de cascade ont conclu qu'il était nécessaire d'utiliser trois gaussiennes. Les résultats de l'ajustement de la distribution de l'équation 5.36 par trois gaussiennes sont montrés dans le tableau 5.14 et dans la figure 5.24.

Les résultats de l'ajustement de la distribution en $\Delta t$ pour les événements étiquetés par un lepton de cascade sont résumés dans le tableau 5.15.

Pour les événements étiquetés par un lepton du $D^{0}$ non reconstruit, on utilise trois gaussiennes pour la fonction de résolution. On ajuste la distribution en $\Delta t$ des événements simulés de signal, étiquetés par un lepton du $D^{0}$, en variant $\tau_{D^{*} \pi}^{\prime}$ et les paramètres de la fonction de résolution. 

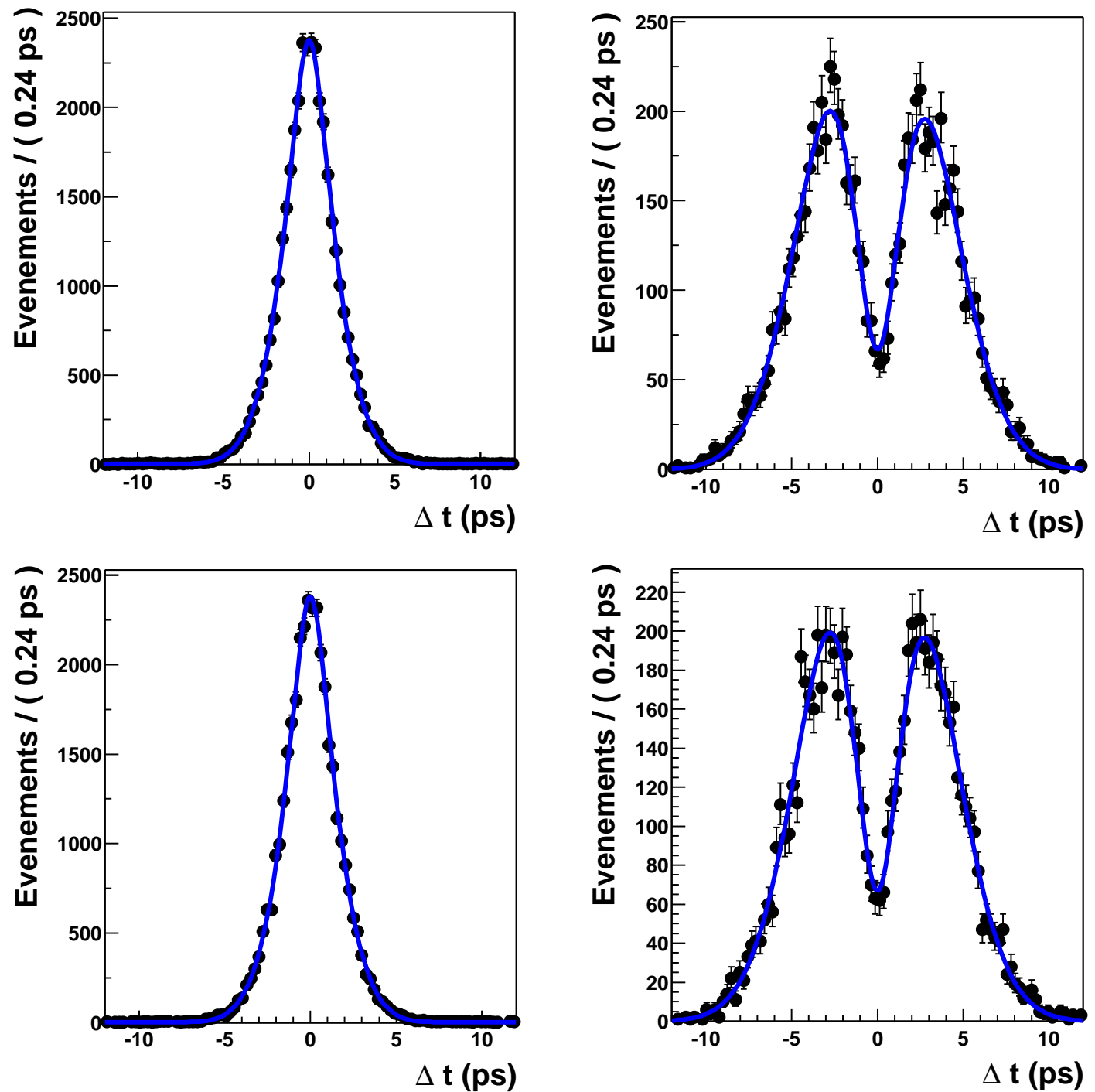

Fig. 5.23: Résultats de l'ajustement de la distribution en $\Delta t$ (avec une fonction de résolution à deux gaussiennes) pour les événements étiquetés par un lepton direct. Les quatre figures, de gauche à droite et de bas en haut correspondent aux événements nonmélangés étiquetés par un $B^{0}$, aux événements mélangés étiquetés par un $B^{0}$, aux événements non-mélangés étiquetés par un $\bar{B}^{0}$, aux événements mélangés étiquetés par un $\bar{B}^{0}$. On remarque que l'ajustement est de bonne qualité. Les niveaux de confiance de l'ajustement sont respectivement $38 \%, 28 \%, 37 \%$ et $29 \%$. 


\begin{tabular}{|c|c|}
\hline$b_{D^{*} \pi}^{n}$ (cas) & $-0,18 \pm 0,02$ \\
$b_{D^{*} \pi}^{w}(\mathrm{cas})$ & $-1,04 \pm 0,12$ \\
\hline$f_{D^{*} \pi}^{n}(\mathrm{cas})$ & $0,84 \pm 0,03$ \\
$f_{D^{*} \pi}^{o}(\mathrm{cas})$ & $0,0037 \pm 0,0004$ \\
\hline$s_{D^{*} \pi}^{n}(\mathrm{cas})$ & $0,969 \pm 0,03$ \\
$s_{D^{*} \pi}^{w}(\mathrm{cas})$ & $1,80 \pm 0,10$ \\
\hline
\end{tabular}

TAB. 5.14: Résultats de l'ajustement par trois gaussiennes de la distribution $\left(\Delta t_{m e s}-\right.$ $\left.\Delta t_{\text {vrai }}\right) / \sigma_{\Delta t}$ pour des événements simulés de signal, étiquetés par un lepton de cascade.

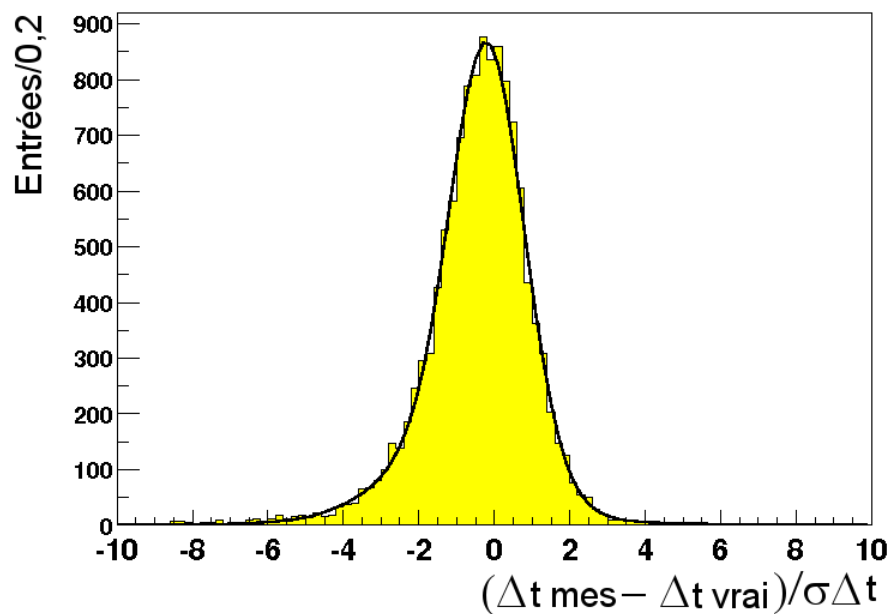

FIG. 5.24: Ajustement par trois gaussiennes de la distribution $\left(\Delta t_{m e s}-\Delta t_{\text {vrai }}\right) / \sigma_{\Delta t}$ pour des événements simulés de signal, étiquetés par un lepton de cascade.

\begin{tabular}{|c|c|}
\hline \multicolumn{2}{|c|}{ Signal, lepton de cascade } \\
\hline$\Delta m_{D^{*} \pi}$ & $0,42 \pm 0,02$ \\
$\tau_{D^{*} \pi}$ & $1,60 \pm 0,03$ \\
$\omega D^{*} \pi(\mathrm{cas})$ & $0,657 \pm 0,006$ \\
\hline$b_{D^{*} \pi}^{n}$ (cas) & $-0,22 \pm 0,061$ \\
$b_{D^{*} \pi}^{w}$ (cas) & $-2,14 \pm 1,18$ \\
$f_{D^{*} \pi}^{n}$ (cas) & $0,90 \pm 0,05$ \\
$f_{D^{*} \pi}^{o}$ (cas) & $0,021 \pm 0,005$ \\
$s_{D^{*} \pi}^{n}$ (cas) & $0,98 \pm 0,03$ \\
$s_{D^{*} \pi}^{w}$ (cas) & $1,81 \pm 0,79$ \\
\hline
\end{tabular}

TAB. 5.15: Résultats de l'ajustement de la distribution en $\Delta t$ pour les événements simulés de signal, étiquetés par un lepton de cascade. 
Ajustement des événements simulés de signal, générés sans violation de $C P$, toutes catégories d'étiquetage par un lepton confondues

Dans l'ajustement global sur l'ensemble des événements simulés de signal, il n'est pas possible d'ajuster simultanément tous les paramètres des trois catégories d'étiquetage (lepton direct, lepton de cascade et lepton provenant du $D^{0}$ non reconstruit). Les paramètres $\Delta m_{D^{*} \pi}, \tau_{D^{*} \pi}, a_{D^{*} \pi}$ et $c_{D^{*} \pi}$ ont la même signification physique pour les événements étiquetés par un lepton direct ou de cascade. On les force donc à être identiques pour ces deux types de signal. En revanche, les fonctions de résolution et les fractions de mauvais étiquetage étant fortement différentes, on les ajuste indépendamment. Cela permet de ne pas biaiser les paramètres de $C P$ mesurés. Pour éviter de trop fortes corrélations dans l'ajustement, on fixe la fraction de mauvais étiquetage des leptons de cascade à la valeur ajustée sur Monte-Carlo. D'autre part, la fraction d'événements étiquetés par un lepton du $D^{0}$ étant très faible (de l'ordre de $1 \%$ ), on peut simplement fixer les paramètres de cette catégorie de signal aux valeurs ajustées sur Monte-Carlo.

La fonction de résolution des événements étiquetés par un lepton direct ne doit pas être biaisée. On a vérifié précédemment que les biais étaient bien compatibles avec 0 . Dans l'ajustement global sur toutes les catégories d'étiquetage par un lepton, on fixe donc ces biais à 0 . On ajuste donc la fraction de gaussienne étroite, et les largeur des deux gaussiennes de la fonction de résolution des événements étiquetés par un lepton direct. En revanche, on s'attend à ce que la fonction de résolution des événements étiquetés par un lepton de cascade soit biaisée. On ajuste donc les biais de la fonction de résolution de ce type d'événements. Les autres paramètres de la fonction de résolution sont fixés aux valeurs trouvée sur Monte-Carlo, afin de limiter le nombre de paramètres corrélés à ajuster.

Le tableau 5.16 résume le traitement de tous les paramètres des PDFs décrivant la distribution de $\Delta t$ et de la fonction de résolution des différentes catégories de signal étiqueté par le $B_{\text {tag }}$.

Les résultats de l'ajustement global sur les événements simulés de signal sont résumés dans le tableau 5.17.

On remarque que les paramètres de $C P$ mesurés sont bien compatibles avec 0 . La valeur pour $\Delta m_{D^{*} \pi}$ est bien compatible avec la valeur générée. Le temps de vie est légèrement biaisé. On le corrige de sa déviation en lui assignant une erreur systématique égale à la moitié de la valeur du biais : $\Delta \tau_{D^{*} \pi}=-0,007 \pm 0,004$. Les autres paramètres ont des valeurs raisonnables. Cet ajustement permet de valider le traitement du signal pour la mesure des paramètres de $C P$, qui sont bien compatibles avec 0 , mais avec une erreur non nulle. On tiendra compte de cette erreur dans les systématiques.

Ajustement des événements simulés de signal, générés avec $r^{*}=0,025$ et $r^{*}=$ 0,1

Les valeurs générées pour les paramètres de $C P$ sont alors :

- $a_{D^{*} \pi}=0,0485$ et $c_{D^{*} \pi}=-0,011$ pour $r^{*}=0,025$. Le résultat de l'ajustement sur tout les événements simulés de signal est résumé dans le tableau 5.18. Les valeurs ajustées de $a_{D^{*} \pi}$ et de $c_{D^{*} \pi}$ sont compatibles avec les valeurs générées $(0,5 \sigma$ pour 


\begin{tabular}{|c|c|c|}
\hline paramètre & ajusté/fixe & catégorie d'étiquetage \\
\hline$a_{D^{*} \pi}$ & ajusté & commun leptons directs et de cascade \\
$c_{D^{*} \pi}$ & ajusté & commun leptons directs et de cascade \\
$\Delta m_{D^{*} \pi}$ & ajusté & commun leptons directs et de cascade \\
$\tau_{D^{*} \pi}$ & ajusté & commun leptons directs et de cascade \\
$\omega_{D^{*} \pi}$ (dir) & ajusté & leptons directs \\
$\omega_{D^{*} \pi}$ (cas) & fixe & leptons de cascade \\
\hline$b_{D^{*} \pi}^{n}$ (dir) & fixe & leptons directs \\
$b_{D^{*} \pi}^{w}($ dir $)$ & fixe & leptons directs \\
$f_{D^{*} \pi}^{n}($ dir $)$ & ajusté & leptons directs \\
$s_{D^{*} \pi}^{n}$ (dir) & ajusté & leptons directs \\
$s_{D^{*} \pi}^{w}$ (dir) & ajusté & leptons directs \\
$b_{D^{*} \pi}^{n}$ (cas) & ajusté & leptons de cascade \\
$b_{D^{*} \pi}^{w}$ (cas) & ajusté & leptons de cascade \\
$f_{D^{*} \pi}^{n}$ (cas) & fixe & leptons de cascade \\
$f_{D^{*} \pi}^{o}$ (cas) & fixe & leptons de cascade \\
$s_{D^{*} \pi}^{n}$ (cas) & fixe & leptons de cascade \\
$s_{D^{*} \pi}^{w}$ (cas) & fixe & leptons de cascade \\
\hline
\end{tabular}

TAB. 5.16: Traitement des paramètres de $\Delta t$ et de la fonction de résolution temporelle du signal étiqueté par un lepton direct ou de cascade dans l'ajustement global du signal.

\begin{tabular}{|c|c|c|}
\hline \multicolumn{3}{|c|}{ Signal (lepton) } \\
\hline Paramètre & valeur ajustée & valeur générée \\
\hline$a D^{*} \pi$ & $0,003 \pm 0,006$ & 0 \\
$c D^{*} \pi$ & $-0,013 \pm 0,007$ & 0 \\
$\Delta m_{D^{*} \pi}$ & $0,488 \pm 0,003$ & 0,489 \\
$\tau_{D^{*} \pi}$ & $1,541 \pm 0,007$ & 1,548 \\
$\omega D^{*} \pi$ (dir) & $0,002 \pm 0,002$ & 0 \\
\hline$b_{D^{*} \pi}^{n}$ (cas) & $-0,30 \pm 0,05$ & \\
$b_{D^{*} \pi}^{w}$ (cas) & $-2,12 \pm 1,36$ & \\
$f_{D^{*} \pi}^{n}$ & $0,972 \pm 0,008$ & \\
$s_{D^{*} \pi}^{n}$ & $0,923 \pm 0,012$ & \\
$s_{D^{*} \pi}^{w}$ & $3,46 \pm 0,46$ \\
\cline { 2 - 3 }
\end{tabular}

ТАВ. 5.17: Résultats de l'ajustement de la distribution en $\Delta t$ pour les événements simulés de signal, étiquetés par un lepton (toutes catégories confondues). 
$a_{D^{*} \pi}$ et $1 \sigma$ pour $\left.c_{D^{*} \pi}\right)$.

\begin{tabular}{|c|c|c|}
\hline \multicolumn{3}{|c|}{ Signal, $r^{*}=0,025$ (lepton) } \\
\hline paramètre & valeur ajustée & valeur générée \\
\hline$a_{D^{*} \pi}$ & $0,0482 \pm 0,006$ & 0,0485 \\
\hline$c_{D^{*} \pi}$ & $-0,014 \pm 0,012$ & $-0,011$ \\
\hline$\Delta m_{D^{*} \pi}$ & $0,481 \pm 0,003$ & 0,489 \\
\hline$\tau_{D^{*} \pi}$ & $1,53 \pm 0,007$ & 1,548 \\
\hline$\omega_{D^{*} \pi}$ (dir) & $0,006 \pm 0,002$ & 0 \\
\hline$b_{D^{*} \pi}^{n}(\operatorname{cas})$ & $-0,29 \pm 0,11$ & \\
\hline$b_{D^{*} \pi}^{w}(\mathrm{cas})$ & $-3,35 \pm 0,97$ & \\
\hline$f_{D^{*} \pi}^{n}$ & $0,97 \pm 0,02$ & \\
\hline$s_{D^{*} \pi}^{n}$ & $0,91 \pm 0,01$ & \\
\hline$s_{D^{*} \pi}^{w}$ & $2,07 \pm 0,22$ & \\
\hline
\end{tabular}

TAB. 5.18: Résultats de l'ajustement de la distribution en $\Delta t$ pour les événements simulés de signal $(r=0,025)$, étiquetés par un lepton (toutes catégories confondues).

- $a_{D^{*} \pi}=0,155$ et $c_{D^{*} \pi}=-0,035$ pour $r^{*}=0,1$. Le résultat de l'ajustement sur tout les événements simulés de signal est résumé dans le tableau 5.19. Les valeurs ajustées de $a_{D^{*} \pi}$ et de $c_{D^{*} \pi}$ sont compatibles avec les valeurs générées ( $0,8 \sigma$ pour $a_{D^{*} \pi}$ et $1 \sigma$ pour $c_{D^{*} \pi}$ ).

\begin{tabular}{|c|c|c|}
\hline \multicolumn{3}{|c|}{ Signal, $r^{*}=0,1$ (lepton) } \\
\hline paramètre & valeur ajustée & valeur générée \\
\hline$a_{D^{*} \pi}$ & $0,145 \pm 0,012$ & 0,155 \\
$c_{D^{*} \pi}$ & $-0,014 \pm 0,021$ & $-0,035$ \\
\hline$\Delta m_{D^{*} \pi}$ & $0,466 \pm 0,006$ & 0,474 \\
$\tau_{D^{*} \pi}$ & $1,562 \pm 0,012$ & 1,548 \\
$\omega_{D^{*} \pi}$ (dir) & $0,009 \pm 0,003$ & 0 \\
\hline$b_{D^{*} \pi}^{n}$ (cas) & $-0,33 \pm 0,10$ & \\
$b_{D^{*} \pi}^{w}$ (cas) & $-3,2 \pm 1,8$ & \\
$f_{D^{*} \pi}^{n}$ & $0,86 \pm 0,08$ & \\
$s_{D^{*} \pi}^{n}$ & $0,94 \pm 0,06$ & \\
$s_{D^{*} \pi}^{w}$ & $1,81 \pm 0,26$ & \\
\cline { 2 - 3 } & \multicolumn{1}{|c}{} \\
\cline { 2 - 3 }
\end{tabular}

ТАВ. 5.19: Résultats de l'ajustement de la distribution en $\Delta t$ pour les événements simulés de signal $(r=0,1)$, étiquetés par un lepton (toutes catégories confondues). Notons que l'échantillon de Monte-Carlo utilisé ici a été généré avec une valeur de $\Delta m$ différente des autres échantillons.

Remarquons de plus que tous les autres paramètres ont des valeurs raisonnables. 


\subsubsection{Validation sur Monte-Carlo pour les événements étiquetés par un kaon}

On utilise les mêmes échantillons de Monte-Carlo que ceux utilisés pour l'analyse des événements étiquetés par un lepton, mais on utilise cette fois-ci les événements étiquetés par un kaon. Cette analyse n'utilise aucun événement en commun avec celle utilisant les événements étiquetés par un lepton et les deux analyses sont totalement indépendantes.

\section{Validation de la procédure avec des événements simulés de signal, générés sans violation de $C P$}

Contrairement au cas des événements étiquetés par un lepton, on ne modélise qu'une catégorie pour les événements de signal. On ajuste les trois paramètres de $C P a_{D^{*} \pi}, b_{D^{*} \pi}$ et $c_{D^{*} \pi}$, ainsi que les paramètres $\Delta m_{D^{*} \pi}, \tau_{D^{*} \pi}, \omega_{D^{*} \pi}, \Delta \omega_{D^{*} \pi}$, ainsi que les paramètres de la fonction de résolution à trois gaussienne. Les résultats sont résumés dans le tableau 5.20 .

\begin{tabular}{|c|c|}
\hline \multicolumn{2}{|c|}{ Signal (kaon) } \\
\hline$a_{D^{*} \pi}$ & $0,0085 \pm 0,0068$ \\
$b_{D^{*} \pi}$ & $0,0005 \pm 0,0033$ \\
$c_{D^{*} \pi}$ & $0,0040 \pm 0,0068$ \\
\hline$\Delta m_{D^{*} \pi}$ & $0,481 \pm 0,002$ \\
$\tau_{D^{*} \pi}$ & $1,509 \pm 0,005$ \\
$\omega_{D^{*} \pi}$ & $0,239 \pm 0,001$ \\
$\Delta \omega_{D^{*} \pi}$ & $-0,011 \pm 0,002$ \\
\hline$b_{D^{*} \pi}^{n}$ & $-0,272 \pm 0,007$ \\
$b_{D^{*} \pi}^{w}$ & $-1,297 \pm 0,119$ \\
$f_{D^{*} \pi}^{n}$ & $0,943 \pm 0,007$ \\
$f_{D^{*} \pi}^{o}$ & $0,003 \pm 0,001$ \\
$s_{D^{*} \pi}^{n}$ & $0,979 \pm 0,012$ \\
$s_{D^{*} \pi}^{w}$ & $3,146 \pm 0,204$ \\
\hline
\end{tabular}

TAB. 5.20: Résultat de l'ajustement des événements simulés de signal, générés avec violation de $C P\left(r^{*}=0,1\right)$ pour les événements étiquetés par un kaon.

Les paramètres de $C P$ sont bien compatibles avec 0 , Notons que temps de vie $\tau_{D^{*} \pi}$ mesuré dans le cas des événements étiquetés par un kaon est un temps de vie effectif. Cela est dû aux événements étiquetés par une trace du $D^{0}$ non reconstruits. Dans l'analyse sur les données, on corrige donc le temps de vie mesuré de la déviation de la valeur de $\tau_{D^{*} \pi}$ mesurée sur Monte-Carlo par rapport à la valeur générée. La correction appliquée est alors $\Delta \tau_{D^{*} \pi}=0,039 \pm 0,020$. Les autres paramètres ont tous des valeurs raisonnables. Cet ajustement permet de valider le traitement du signal pour la mesure des paramètres de $C P$, qui sont bien compatibles avec 0 , mais avec une erreur non nulle. On tiendra compte de cette erreur dans les systématiques. 
Ajustement des événements simulés de signal, générés avec $r^{*}=0,1$

Le Monte-Carlo a été généré sans violation de $C P$ du côté de $B_{\text {tag }}$, c'est-à-dire avec $r^{\prime}=0$. Les valeurs générées pour les paramètres de $C P$ sont alors :

$-a_{D^{*} \pi}=0,155, b_{D^{*} \pi}=0$ et $c_{D^{*} \pi}=-0,035$, avec $r^{*}=0,1$.

Les résultats des ajustements sur tout les événements simulés de signal sont résumés dans le tableau 5.21.

\begin{tabular}{|c|c|}
\hline \multicolumn{2}{|c|}{ Signal, $r^{*}=0,1$ (kaon) } \\
\hline$a_{D^{*} \pi}$ & $0,148 \pm 0,014$ \\
$b_{D^{*} \pi}$ & $0,001 \pm 0,007$ \\
$c_{D^{*} \pi}$ & $-0,048 \pm 0,014$ \\
\hline$\Delta m_{D^{*} \pi}$ & $0,453 \pm 0,006$ \\
$\tau_{D^{*} \pi}$ & $1,526 \pm 0,009$ \\
$\omega_{D^{*} \pi}$ & $0,237 \pm 0,002$ \\
$\Delta \omega_{D^{*} \pi}$ & $-0,011 \pm 0,005$ \\
\hline$b_{D^{*} \pi}^{n}$ & $-0,279 \pm 0,011$ \\
$b_{D^{*} \pi}^{w}$ & $-0,852 \pm 0,245$ \\
$f_{D^{*} \pi}^{n}$ & $0,958 \pm 0,012$ \\
$f_{D^{*} \pi}^{o}$ & $0,003 \pm 0,001$ \\
$s_{D^{*} \pi}^{n}$ & $1,043 \pm 0,021$ \\
$s_{D^{*} \pi}^{w}$ & $3,480 \pm 0,442$ \\
\hline
\end{tabular}

TAB. 5.21: Résultat de l'ajustement des événements simulés de signal, générés avec violation de $C P\left(r^{*}=0,1\right)$ pour les événements étiquetés par un kaon.

Les paramètres de $C P$ ajustés sont bien compatibles avec les paramètres générés.

\subsubsection{Conclusions de la validation sur Monte-Carlo}

La procédure pour traiter les événements de signal est bien établie. On n'a pas observé de biais sur l'ajustement des paramètres de $C P$. On a cependant observé un biais sur l'ajustement de $\tau_{D^{*} \pi}$, dont l'origine est comprise. On est capable de corriger ce biais et on a estimé l'erreur systématique correspondante. Notons que la corrélation entre $\tau_{D^{*} \pi}$ et les paramètres de $C P$ est très faible (de l'ordre de quelques \%) et donc ce biais n'affecte en rien la mesure des paramètres de $C P$. Cela a été confirmé par des tests dans lesquels on a fixé $\tau_{D^{*} \pi}$ à la valeur générée. L'impact sur l'ajustement des paramètres de $C P$ s'est révélé être totalement négligeable.

Il s'agit maintenant de traiter les différents bruits de fond et d'évaluer leur impact sur la mesure réalisée. Dans un premier temps, on modélise les formes des PDFs en $\Delta t$ des différents types de bruit de fond $B \bar{B}$ sur Monte-Carlo, puis on les ajoute un à un aux événements de signal afin d'étudier leur impact sur l'ajustement des paramètres du signal. La procédure utilisée ainsi que sa validation sont exposées dans la partie suivante. 


\title{
5.6 Analyse en $\Delta t$ des bruits de fond
}

\subsubsection{PDFs des bruits de fond}

\author{
$\mathrm{PDF}$ en $\Delta t$ de $D^{*} \rho$
}

La PDF en $\Delta t$ des événements $B^{0} \rightarrow D^{*-} \rho^{+}$a la même forme fonctionnelle et les mêmes paramètres que la $\mathrm{PDF}$ du signal $B^{0} \rightarrow D^{*-} \pi^{+}$, à la différence près que les paramètres de $C P$ de $B^{0} \rightarrow D^{*-} \rho^{+}$sont fixés à 0 . En effet, les paramètres $\Delta m, \tau$ et $\omega$ ont la même signification physique pour $D^{*} \pi$ et $D^{*} \rho$ et la fonction de résolution est comparable. Cependant, on cherche à mesurer les asymétries de $C P$ dans la désintégration $B^{0} \rightarrow D^{*-} \pi^{+}$, et on ne peut donc pas utiliser les mêmes paramètres de $C P$ pour $D^{*} \pi$ et pour $D^{*} \rho$. En particulier, le rapport $r^{*}$ et les phases fortes peuvent être différentes pour les événements $B^{0} \rightarrow D^{*-} \rho^{+}$. Puisqu'à l'heure actuelle nous ne disposons pas de mesure des paramètres de $C P$ dans les désintégrations $D^{*} \rho$, l'analyse a été faite en fixant ces paramètres de $C P$ à 0 . Nous avons ensuite estimé la systématique associée de la façon décrite dans la partie 5.8 .

La validité de l'utilisation des mêmes paramètres pour la fonction de résolution de $D^{*} \pi$ et de $D^{*} \rho$ a été vérifiée avec des événements simulés. L'explication physique est que le spectre de l'impulsion du pion dur des événements $B^{0} \rightarrow D^{*-} \rho^{+}\left(\operatorname{avec} \rho^{+} \rightarrow \pi^{+} \pi^{0}\right)$ qui passent la sélection est quasiment identique à celui du signal $B^{0} \rightarrow D^{*-} \pi^{+}$.

\section{$\mathrm{PDF}$ en $\Delta t \mathrm{du}$ bruit de fond qui pique en masse manquante}

On distingue deux types de bruit de fond qui pique : les événements qui proviennent de la désintégration de $B$ chargés $\left(B^{+}\right)$et ceux qui proviennent de la désintégration de $B$ neutres $\left(B^{0}\right)$. La PDF de ces derniers a la même forme fonctionnelle que celle du signal.

En revanche, la PDF du bruit de fond qui pique provenant de $B$ chargés est différente. En effet, il n'y a pas lieu de parler de mélange pour ce type d'événement et il n'y a donc pas de terme en $\cos (\Delta m \Delta t)$. La PDF $\mathcal{T}_{\text {peakB }^{ \pm}}$utilisée est alors une exponentielle décroissante de la forme :

$$
\mathcal{T}_{\text {peakB }^{ \pm}}=\frac{1}{2 \tau_{\text {peakB }^{ \pm}}}\left(1-S_{\text {tag }} \Delta \omega_{\text {peakB }^{ \pm}}+S_{\text {mix }}\left(1-2 \omega_{\text {peakB }^{ \pm}}\right)\right) e^{\frac{-\left|\Delta t_{\text {vrai }}\right|}{\tau_{\text {peakB }}^{ \pm}}} .
$$

On note $f_{\text {peakB }}{ }$ la fraction de bruit de fond qui pique provenant de $B$ chargés par rapport à l'ensemble du bruit de fond qui pique. Il n'est pas possible d'ajuster les paramètres de la $\mathrm{PDF}$ en $\Delta t$ du bruit de fond qui pique sur les données. Tous les paramètres sont donc ajustés sur Monte-Carlo.

\section{$\mathrm{PDF}$ en $\Delta t \mathrm{du}$ bruit de fond combinatoire}

Les paramètres de la $\mathrm{PDF}$ en $\Delta t$ du bruit de fond combinatoire $\mathcal{T}_{\text {comb }}$ peuvent être ajustés directement sur les données, en utilisant la région latérale (voir partie 5.3). Il convient donc d'avoir une paramétrisation suffisamment simple pour qu'un minimum de paramètres utilisés provienne du Monte-Carlo. Ainsi, on ne distingue pas les désintégrations de $B^{0}$ 
des désintégrations de $B^{+}$, ce qui est une bonne approximation tant que leurs durées de vie ne sont pas trop différentes $\left(\tau_{B}^{0}=1,436 \pm 0,014 \mathrm{ps}\right.$ et $\left.\tau_{B^{ \pm}}=1,671 \pm 0,018 \mathrm{ps}\right)$.

La validité de cette approximation a été estimée grâce à des tests de KolmogorovSmirnov réalisés sur Monte-Carlo. Ces tests donnent la probabilité de la compatibilité entre les distributions en $\Delta t$ du combinatoire provenant de $B^{0}$ et du combinatoire provenant de $B^{+}$. Cette probabilité est de 40,43\% dans la région du signal et de 31,24\% dans la région latérale dans laquelle on mesure les paramètres de la PDF en $\Delta t$ du combinatoire. Il est donc totalement justifié de n'utiliser qu'une seule PDF pour décrire les deux types de combinatoire. La PDF utilisée a la même forme fonctionnelle que la PDF du signal. Notons que pour les événements étiquetés par un lepton, on ne distingue pas les différents types de leptons étiquetant.

D'autre part, contrairement au cas du signal, on ne peut pas déterminer directement les paramètres $\alpha$ et $\rho$. Il existe une paramétrisation qui consiste à réduire les quatre paramètres $\alpha_{\text {comb }}, \rho_{\text {comb }}, \omega_{\text {comb }}$ et $\Delta \omega_{\text {comb }}$, en trois notés $\mathcal{O}_{\text {comb }}, \omega_{\text {comb }}^{\prime}$ et $\Delta \omega_{\text {comb }}^{\prime}$ et qui s'écrivent ainsi en fonction des précédents :

$$
\begin{aligned}
\omega_{\mathrm{comb}}^{\prime} & =\omega_{\mathrm{comb}}\left(1-\alpha_{\mathrm{comb}}\right)+\frac{\alpha_{\mathrm{comb}}}{2}, \\
\Delta \omega_{\mathrm{comb}}^{\prime} & =\Delta \omega_{\mathrm{comb}}\left(1-\alpha_{\mathrm{comb}}\right) \\
\mathcal{O}_{\mathrm{comb}} & =\alpha_{\mathrm{comb}}\left(1-\rho_{\mathrm{comb}}\right) .
\end{aligned}
$$

La PDF $\mathcal{T}_{\text {comb }}^{\prime}$ s'écrit alors en fonction de ces paramètres de la façon suivante :

$$
\begin{aligned}
\mathcal{T}_{\text {comb }}^{\prime}\left(\Delta t_{\text {vrai }}, S_{\text {mix }}, S_{\text {tag }}\right)= & \frac{1}{8 \tau} \exp \left(-\frac{\left|\Delta t_{\text {vrai }}\right|}{\tau}\right) \\
& {\left[\left(1-S_{\text {tag }} \Delta \omega^{\prime}+S_{\text {mix }} \mathcal{O}\right)+S_{\text {mix }}\left(1-2 \omega^{\prime}\right) A \cos \left(\Delta m \Delta t_{\text {vrai }}\right)\right.} \\
& \left.-S_{\text {tag }}\left(1-2 \omega^{\prime}\right) B \sin (2 \beta+\gamma+\delta) \sin \left(\Delta m \Delta t_{\text {vrai }}\right)\right] .
\end{aligned}
$$

\section{$\mathrm{PDF}$ en $\Delta t \mathrm{du}$ continuum}

Le continuum correspond aux événements de type $e^{+} e^{-} \rightarrow q \bar{q}$, avec $q=u, d, s, c$. La PDF du continuum est la somme d'une fonction de Dirac $\delta$, qui permet de décrire les événements de continuum qui ont un $\Delta t_{\text {vrai }}=0$ et d'une exponentielle décroissante, qui permet de décrire les événements avec un $\Delta t_{\text {vrai }} \neq 0$. La forme fonctionnelle est la suivante :

$$
\mathcal{T}_{q \bar{q}}=\left[f^{\delta} \delta\left(\Delta t_{\text {vrai }}\right)+\left(1-f^{\delta}\right) \frac{1}{2 \tau_{q \bar{q}}} e^{\frac{-\left|\Delta t_{\text {va } a i}\right|}{\tau_{q \bar{q}}}}\right]\left(1-S_{\mathrm{tag}} \Delta \omega_{q \bar{q}}+S_{\text {mix }}\left(1-2 \omega_{q \bar{q}}\right)\right)
$$

où $f^{\delta}$ représente la fraction de fonction $\delta, \tau_{q \bar{q}}$ un temps de vie effectif et $\omega_{q \bar{q}}$ une fraction de mauvais étiquetage effectif. 


\section{Fonctions de résolution des $\mathrm{PDF}$ en $\Delta t$ des bruits de fond}

Chacune des PDFs des bruits de fond décrites précédemment est convoluée avec une fonction de résolution à trois gaussiennes de la même forme que celle du signal, mais avec des paramètres différents.

\subsubsection{Validation sur Monte-Carlo du traitement des bruits de fond pour les événements étiquetés par un lepton}

\section{Ajustement du bruit de fond combinatoire sur Monte-Carlo}

Pour cette étude, on utilise l'échantillon de $B^{0}$ génériques dans la région latérale en $m_{\text {rec }}\left(1,81 \mathrm{GeV}<m_{\text {rec }} 1,84 \mathrm{GeV}\right)$. En effet, dans cette région, il n'y a que du bruit de fond combinatoire et du continuum. Utiliser la région latérale nous permet donc d'ajuster les paramètres du combinatoire sur les données.

Ainsi que cela a été expliqué dans la section 5.6, on utilise une seule PDF pour modéliser la distribution en $\Delta t$ du combinatoire issu de $B^{0}$ et de $B^{+}$dans la région latérale. On ajuste les paramètres effectifs $\Delta m_{\mathrm{comb}}, \tau_{\mathrm{comb}}, \mathcal{O}_{\mathrm{comb}}$ et $\omega_{\mathrm{comb}}$ et la fonction de résolution. Les résultats sont résumés dans le tableau 5.22.

\begin{tabular}{|c|c|}
\hline \multicolumn{2}{|c|}{ Bruit de fond combinatoire } \\
\hline$\Delta m_{\text {comb }}$ & $0,51 \pm 0,02$ \\
$\tau_{\text {comb }}$ & $1,64 \pm 0,01$ \\
$\mathcal{O}_{\text {comb }}$ & $0,39 \pm 0,01$ \\
$\omega_{\text {comb }}$ & $0,38 \pm 0,01$ \\
\hline$b_{\text {comb }}^{n}$ & $0,01 \pm 0,02$ \\
$b_{\text {comb }}^{w}$ & $-0,37 \pm 0,15$ \\
$f_{\text {comb }}^{n}$ & $0,84 \pm 0,32$ \\
$f_{\text {comb }}^{o}$ & $0,01 \pm 0,002$ \\
$s_{\text {comb }}^{n}$ & $0,81 \pm 0,02$ \\
$s_{\text {comb }}^{w}$ & $1,87 \pm 0,15$ \\
\hline
\end{tabular}

TAB. 5.22: Ajustement du bruit de fond combinatoire sur Monte-Carlo dans la région latérale en $m_{\text {rec }}$.

On a vérifié la bonne compatibilité sur Monte-Carlo entre les paramètres du bruit de fond combinatoire dans la région latérale et dans la région du signal, ce qui nous permet d'utiliser les paramètres ajustés dans la région latérale pour modéliser le bruit de fond combinatoire dans la région du signal.

\section{Ajustement du bruit de fond qui pique sur Monte-Carlo}

On ajuste séparément les distributions en $\Delta t$ du bruit de fond qui pique issu de $B^{0}$ et de $B^{+}$. Pour la première catégorie, on distingue les différents types de leptons étiquetants. En effet, puisque tous les paramètres du bruit de fond qui pique sont issus du Monte-Carlo, on n'est pas limité par le nombre de paramètres que l'on ajuste. Les paramètres ajustés 
sont résumés dans le tableau 5.23 pour le bruit de fond qui pique $B^{0}$ et dans le tableau 5.24 pour le bruit de fond qui pique $B^{+}$.

\begin{tabular}{|c|c|c|c|}
\cline { 2 - 4 } \multicolumn{1}{c|}{} & \multicolumn{3}{|c|}{ Bruit de fond qui pique $B^{0}$} \\
\cline { 2 - 4 } \multicolumn{1}{c|}{} & lepton direct & lepton de cascade & lepton du $D^{0}$ \\
\hline$\Delta m_{\text {peakB }^{0}}$ & $0,476 \pm 0,006$ & $0,405 \pm 0,055$ & \\
$\tau_{\text {peakB }}$ & $1,534 \pm 0,018$ & $1,594 \pm 0,075$ & $0,568 \pm 0,135$ \\
$\omega_{\text {peakB }}$ & $0,007 \pm 0,003$ & $0,658 \pm 0,016$ & \\
$O_{\text {peakB }}$ & & & $-0,098 \pm 0,054$ \\
\hline$b_{\text {peakB }}^{n}$ & $-0,072 \pm 0,025$ & $-0,299 \pm 0,081$ & $-0,453 \pm 0,149$ \\
$b_{\text {peakB }}^{w}$ & $0,066 \pm 0,287$ & $-3.252 \pm 2.220$ & $-0,685 \pm 1.400$ \\
$f_{\text {peakB }}^{n}$ & $0,925 \pm 0,027$ & $0,928 \pm 0,054$ & $0,849 \pm 1.646$ \\
$f_{\text {peakB }}^{o}$ & $0,000 \pm 0,003$ & $0,029 \pm 0,017$ & $0,078 \pm 0,051$ \\
$s_{\text {peakB }}^{n}$ & $0,834 \pm 0,038$ & $0,899 \pm 0,073$ & $0,668 \pm 0,178$ \\
\hline
\end{tabular}

TAB. 5.23: Ajustement des distributions en $\Delta t$ du bruit de fond qui pique issu de mésons $B^{0}$ $\left(\right.$ peakB $\left.{ }^{0}\right)$ pour différentes catégories d'étiquetage par un lepton. L'échantillon de Monte-Carlo comprend 12571 leptons directs, 1725 leptons de cascade et 145 leptons issus du $D^{0}$ non reconstruit.

\begin{tabular}{|c|c|}
\cline { 2 - 3 } \multicolumn{1}{c|}{} & Bruit de fond qui pique $B^{0}$ \\
\hline$\tau_{\text {peakB }^{ \pm}}$ & $1,664 \pm 0,033$ \\
$\omega_{\text {peakB }}$ & $0,063 \pm 0,003$ \\
\hline$b_{\text {peakB }}^{n}$ & $-0,098 \pm 0,036$ \\
$b_{\text {peakB }}^{w}$ & $-3,396 \pm 0,783$ \\
$f_{\text {peakB }}^{n}$ & $0,973 \pm 0,013$ \\
$f_{\text {peakB }}^{o}$ & $0,000 \pm 0,004$ \\
$s_{\text {peakB }}^{n}$ & $0,747 \pm 0,042$ \\
$s_{\text {peakB }}^{w}$ & $4,039 \pm 0,914$ \\
\hline
\end{tabular}

ТАВ. 5.24: Ajustement de la distribution en $\Delta t$ du bruit de fond qui pique issu de mésons $B^{+}$ $\left(\right.$ peakB $\left.{ }^{ \pm}\right)$. L'échantillon de Monte-Carlo comprend 6163 événements.

\section{Effet du bruit de fond sur l'ajustement du signal Monte-Carlo}

On cherche à tester l'effet du bruit de fond sur l'ajustement des paramètres du signal Monte-Carlo. Pour ce faire, on utilise les échantillons de $B^{0}$ et de $B^{+}$génériques. On ajuste la distribution en $\Delta t$ du signal seul, puis on ajoute les différentes contributions de bruit de fond. On ajuste alors à nouveau les paramètres du signal Monte-Carlo et on regarde la déviation des paramètres et de leurs erreurs. Les fractions des différents types d'événements sont obtenues par comptage sur Monte-Carlo. L'impact du bruit de fond combinatoire et du bruit de fond qui pique a été testé séparément dans un premier temps, 
puis on a testé l'impact global du bruit de fond $B \bar{B}$. Les variations des paramètres de $C P$ sous l'ajout des bruits de fond sont résumés dans le tableau 5.25. L'erreur sur la variation est la différence quadratique entre l'erreur de l'ajustement avec bruit de fond et l'erreur de l'ajustement sans bruit de fond. En effet, l'ajout de bruit de fond augmente l'erreur sur les paramètres de $C P$. On constate que les déviations des paramètres de $C P$ sont compatibles avec 0 , ce qui valide le traitement du bruit de fond sur Monte-Carlo.

\begin{tabular}{|c|c|c|}
\cline { 2 - 3 } \multicolumn{1}{c|}{} & $\Delta\left(a_{D^{*} \pi}\right)$ & $\Delta\left(c_{D^{*} \pi}\right)$ \\
\hline$D^{*} \pi+$ peak & $0,003 \pm 0,003$ & $0,010 \pm 0,009$ \\
$D^{*} \pi+$ comb & $0,004 \pm 0,003$ & $0,004 \pm 0,011$ \\
$D^{*} \pi+$ peak + comb & $0,003 \pm 0,006$ & $0,016 \pm 0,012$ \\
\hline
\end{tabular}

TAB. 5.25: Effet des différents types de bruit de fond sur l'ajustement des paramètres de $C P$ du signal Monte-Carlo.

D'autre part, bien que l'on ne cherche pas à mesurer les paramètres $\Delta m_{D^{*} \pi}$ et $\tau_{D^{*} \pi}$, il est intéressant de regarder l'effet du bruit de fond sur l'ajustement de ces paramètres. Les valeurs ajustées de $\Delta m_{D^{*} \pi}$ et $\tau_{D^{*} \pi}$ sous l'effet du bruit de fond sont résumées dans le tableau 5.26.

\begin{tabular}{|c|c|c|}
\cline { 2 - 3 } \multicolumn{1}{c|}{} & $\Delta\left(\Delta m_{D^{*} \pi}\right)$ & $\Delta\left(\tau_{D^{*} \pi}\right)$ \\
\hline$D^{*} \pi+$ peak & 0,009 & $-0,008$ \\
$D^{*} \pi+$ comb & $-0,009$ & $-0,018$ \\
$D^{*} \pi+$ peak + comb & 0,002 & $-0,026$ \\
\hline
\end{tabular}

TAB. 5.26: Effet des différents types de bruit de fond sur l'ajustement des paramètres $\Delta m_{D^{*} \pi}$ et $\tau_{D^{*} \pi}$ sur Monte-Carlo.

Le bruit de fond augmente très peu l'erreur sur les paramètres $\Delta m_{D^{*} \pi}$ et $\tau_{D^{*} \pi}$ : on n'a donc pas attribué d'erreur sur la variation de ces paramètres sous l'ajout du bruit de fond. On remarque que le bruit de fond n'affecte pas la mesure de $\Delta m_{D^{*} \pi}$. En revanche, la valeur de $\tau_{D^{*} \pi}$ est biaisée, en particulier par le bruit de fond combinatoire. En pratique, on corrige la valeur de $\tau_{D^{*} \pi}$ mesurée sur les données de la déviation observée sur Monte-Carlo et on assigne une erreur systématique égale à la moitié de la valeur du biais. La correction appliquée est donc $\Delta \tau_{D^{*} \pi}=-0,026 \pm 0,013$.

\subsubsection{Validation sur Monte-Carlo du traitement des bruits de fond pour les événements étiquetés par un kaon}

\section{Ajustement du bruit de fond combinatoire dans la région latérale}

Effet du bruit de fond sur la mesure des paramètres de $C P$ pour les événements étiquetés par un kaon

On réalise la même procédure que pour les événements étiquetés par un lepton afin de voir l'impact des différents bruits de fond sur la mesure des paramètres de $C P$. 


\begin{tabular}{|c|c|}
\hline \multicolumn{2}{|c|}{ Combinatoire } \\
\hline$\Delta m_{\text {combB }}$ & $0,582 \pm 0,022$ \\
$\tau_{\text {combB }}$ & $1,694 \pm 0,007$ \\
$\omega_{\text {combB }}$ & $0,456 \pm 0,002$ \\
$\tau_{\text {combB }}$ & $1,817 \pm 0,032$ \\
$O_{\text {combB }}$ & $0,289 \pm 0,004$ \\
\hline$b_{\text {combB }}^{n}$ & $-0,239 \pm 0,009$ \\
$b_{\text {combB }}^{w}$ & $1,324 \pm 0,179$ \\
$f_{\text {combB }}^{n}$ & $0,941 \pm 0,010$ \\
$f_{\text {combB }}^{o}$ & $0,007 \pm 0,001$ \\
$s_{\text {combB }}^{n}$ & $0,936 \pm 0,014$ \\
$s_{\text {combB }}^{w}$ & $2,793 \pm 0,260$ \\
\hline
\end{tabular}

TAB. 5.27: Résultat de l'ajustement du bruit de fond combinatoire provenant du Monte-Carlo générique pour des événements étiquetés par un kaon dans la région latérale.

Les variations de ces paramètres sous l'effet des différents bruits de fond sont résumées dans le tableau 5.28 .

\begin{tabular}{|c|c|c|c|}
\cline { 2 - 4 } \multicolumn{1}{c|}{} & $\Delta a_{D^{*} \pi}$ & $\Delta b_{D^{*} \pi}$ & $\Delta c_{D^{*} \pi}$ \\
\hline signal + comb & $0,004 \pm 0,007$ & $0,002 \pm 0,007$ & $0,0024 \pm 0,007$ \\
signal + comb + peak & $0,002 \pm 0,010$ & $0,006 \pm 0,010$ & $0,007 \pm 0,010$ \\
\hline
\end{tabular}

TAB. 5.28: Effet des différents types de bruit de fond sur l'ajustement des paramètres de $C P$ du signal Monte-Carlo.

On remarque que la déviation des paramètres de $C P$ est compatible avec les erreurs. De même que pour l'analyse des événements étiquetés par un lepton, le bruit de fond a tendance à biaiser le temps de vie $\tau_{D^{*} \pi}$. De plus, on utilise le même temps de vie

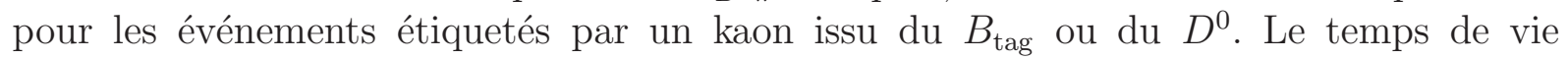
mesuré est donc un temps de vie effectif. On corrige alors le temps de vie mesuré sur les données de la déviation due au bruit de fond observée sur Monte-Carlo et on assigne une erreur systématique égale à la moitié de la correction. La correction appliquée est $\Delta \tau_{D^{*} \pi}=+0,04 \pm 0,02 p s$.

\subsubsection{Conclusion de l'étude sur Monte-Carlo}

En résumé, on peut conclure de toutes les études réalisées sur Monte-Carlo que le signal est bien modélisé et que les bruits de fond n'affectent pas la mesure des paramètres de $C P$. Notons cependant que les événements de bruit de fond ont été générés sans violation de $C P$ et que leurs paramètres de $C P$ ont été fixés à 0 dans toute l'étude. Les paramètres de $C P$ $\mathrm{du}$ bruit de fond sont également fixés à 0 dans l'ajustement sur les données. Cependant, on ne connaît pas le contenu en violation de $C P$ de ces bruits de fond et il faudra donc 
tenir compte de la présence éventuelle de violation de $C P$ dans le bruit de fond dans les erreurs systématiques (voir section 5.8.3).

On a également remarqué que le temps de vie ajusté est biaisé. Les sources de ce biais sont bien comprises. On corrigera donc le temps de vie mesuré sur les données des biais observés sur Monte-Carlo et on assignera une erreur systématique à la mesure, de la façon exposée précédemment.

En conclusion, l'étude sur Monte-Carlo a permis de valider l'analyse. Il s'agit maintenant d'appliquer la même procédure aux données.

\subsection{Résultats de l'analyse}

\subsubsection{Résultats de l'analyse en $\Delta t$ pour les événements étiquetés par un lepton}

L'analyse a été réalisée en utilisant toutes les données disponibles à l'été 2004. Cela correspond à une luminosité de $211 \mathrm{fb}^{-1}$ à la résonance $\Upsilon(4 S)$ et de $21 \mathrm{fb}^{-1}$ sous la résonance. On ajuste simultanément les distributions en $\Delta t$ de ces deux types de données.

On commence par ajuster le bruit de fond combinatoire et le continuum dans la région latérale en $m_{\text {rec }}$, puis on utilise ces paramètres pour ajuster le signal ainsi que le continuum dans la région du signal. Le bruit de fond qui pique se trouvant quasi-intégralement dans la région du signal, il n'est pas possible d'ajuster ses paramètres sur les données, car ils seraient trop corrélés à ceux du signal. On utilise donc les valeurs des paramètres ajustés sur Monte-Carlo pour les PDFs du bruit de fond qui pique.

\section{Ajustement en $\Delta t$ dans la région latérale}

La distribution en $\Delta t$ des données est ajustée dans la région latérale $(1,81 \mathrm{GeV}<$ $\left.m_{\text {rec }}<1,84 \mathrm{GeV}\right)$. Dans cette région, il n'y a, avec une très bonne approximation, que du bruit de fond combinatoire et du continuum. La fraction de continuum pour les données prises à la résonance est calculée en utilisant l'équation 5.15. Les résultats sont résumés dans le tableau 5.29 et la figure correspondante est montrée figure 5.25 en échelle linéaire et figure 5.26 en échelle logarithmique. Les probabilités de Kolmogorov-Smirnov pour les quatre figures sont respectivement, de gauche à droite et de haut en bas, $98 \%, 52 \%$, $37 \%$ et $91 \%$. L'ajustement est donc de bonne qualité. 


\begin{tabular}{|c|c|}
\hline \multicolumn{2}{|c|}{$\begin{array}{l}\text { Données, région latérale } \\
\text { Étiquetage par un lepton }\end{array}$} \\
\hline \multicolumn{2}{|c|}{ Combinatoire } \\
\hline$\Delta m_{\mathrm{comb}}\left(p s^{-1}\right)$ & $0,375 \pm 0,043$ \\
\hline$\tau_{\text {comb }}(p s)$ & $1,542 \pm 0,026$ \\
\hline$\omega_{\mathrm{comb}}$ & $0,31 \pm 0,04$ \\
\hline $\mathcal{O}_{\text {comb }}$ & $0,36 \pm 0,02$ \\
\hline$b_{\mathrm{comb}}^{n}$ & $-0,14 \pm 0,22$ \\
\hline$b_{\mathrm{comb}}^{w}$ & $0,05 \pm 0,11$ \\
\hline$f_{\mathrm{comb}}^{n}$ & $0,74 \pm 0,36$ \\
\hline$f_{\mathrm{comb}}^{o}$ & $0,012 \pm 0,005$ \\
\hline$s_{\mathrm{comb}}^{n}$ & $0,93 \pm 0,13$ \\
\hline$s_{\mathrm{comb}}^{w}$ & $1,56 \pm 0,75$ \\
\hline \multicolumn{2}{|c|}{ Continuum } \\
\hline$\tau_{q \bar{q}}(p s)$ & $1,29 \pm 0,25$ \\
\hline$\omega_{q \bar{q}}$ & $0,368 \pm 0,007$ \\
\hline$f_{q \bar{q}}^{\delta}$ & $0,84 \pm 0,04$ \\
\hline$b_{q \bar{q}}^{n}$ & $-0,035 \pm 0,025$ \\
\hline$b_{q \bar{q}}^{w}$ & $-0,15 \pm 0,21$ \\
\hline$f_{q \bar{q}}^{n}$ & $0,80 \pm 0,05$ \\
\hline$f_{q \bar{q}}^{o}$ & $0,05 \pm 0,01$ \\
\hline$s_{q \bar{q}}^{n}$ & $1,00 \pm 0,03$ \\
\hline$s_{q \bar{q}}^{w}$ & $2,28 \pm 0,35$ \\
\hline
\end{tabular}

TAB. 5.29: Ajustement de la distribution en $\Delta t$ dans la région latérale pour des événements étiquetés par un lepton 

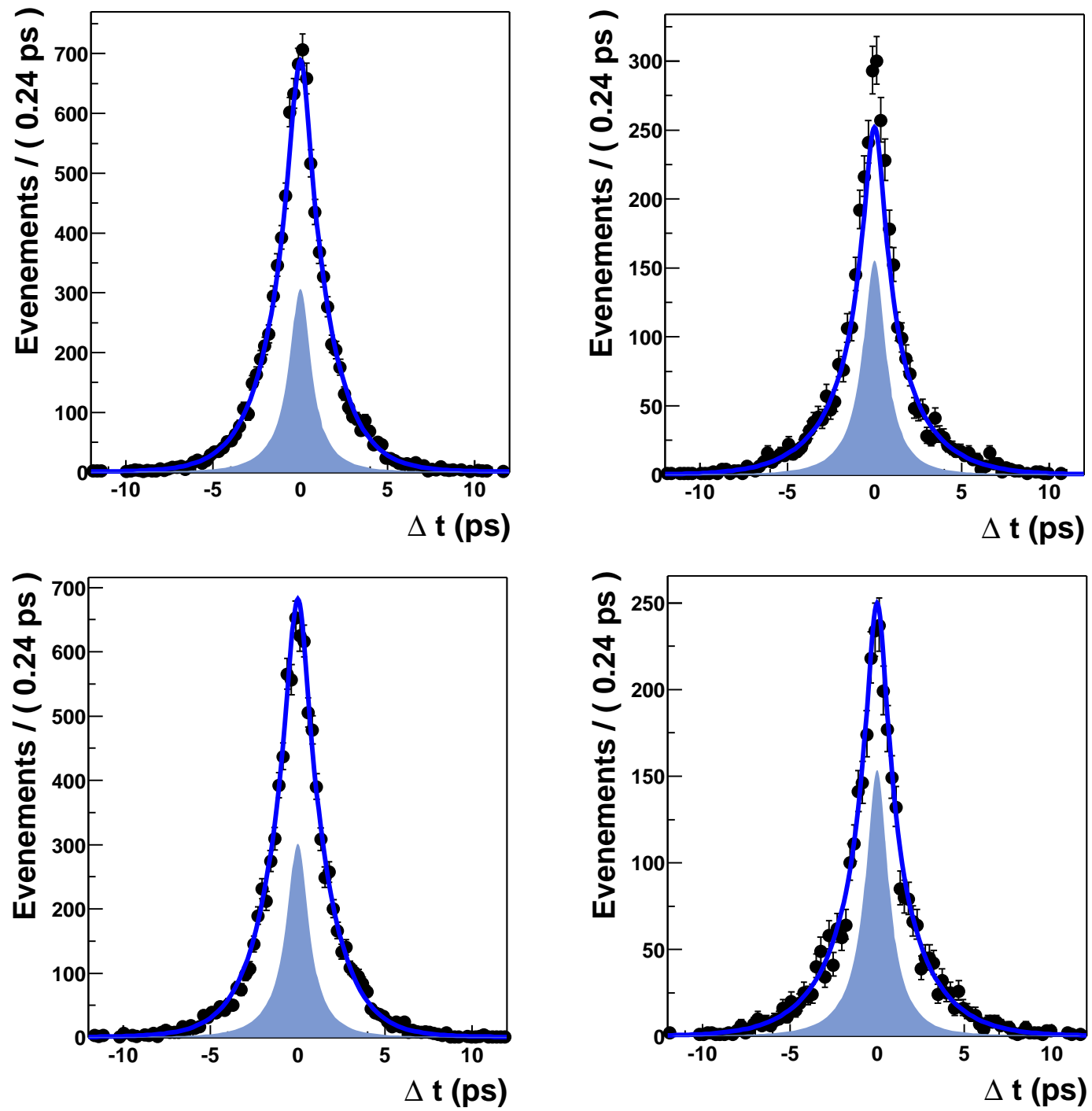

Fig. 5.25: Résultat de l'ajustement sur les données dans la région latérale pour les événements étiquetés par un lepton. Les quatre figures, de gauche à droite et de bas en haut correspondent aux événements non-mélangés étiquetés par un $B^{0}$, aux événements mélangés étiquetés par un $B^{0}$, aux événements non-mélangés étiquetés par un $\bar{B}^{0}$, aux événements mélangés étiquetés par un $\bar{B}^{0}$. Les courbes montrent de bas en haut les contributions cumulées du continuum et du combinatoire. 

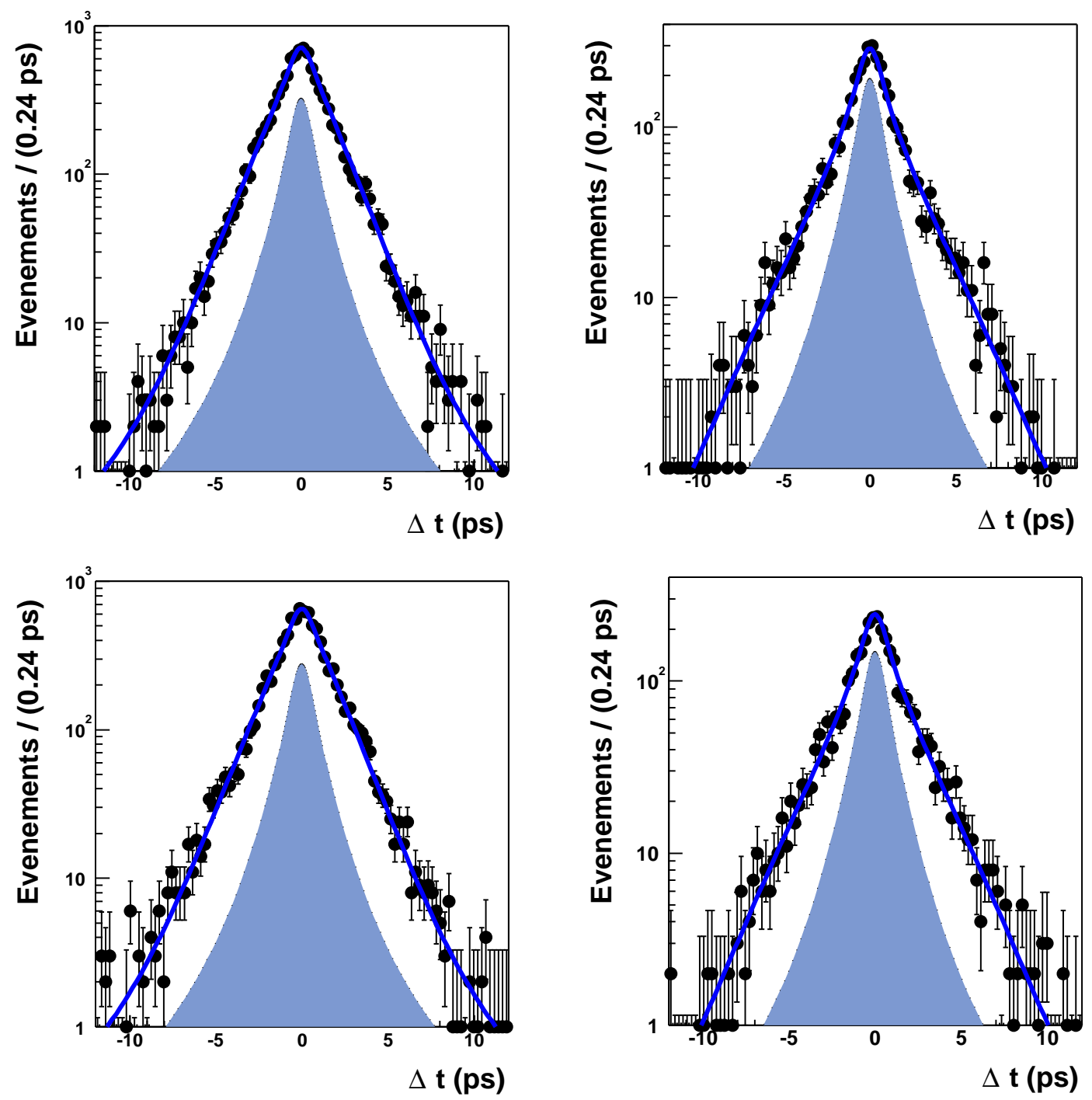

FIG. 5.26: Résultat de l'ajustement sur les données en échelle logarithmique dans la région latérale pour les événements étiquetés par un lepton. Les quatre figures, de gauche à droite et de bas en haut correspondent aux événements non-mélangés étiquetés par un $B^{0}$, aux événements mélangés étiquetés par un $B^{0}$, aux événements non-mélangés étiquetés par un $\bar{B}^{0}$, aux événements mélangés étiquetés par un $\bar{B}^{0}$. Les courbes montrent de bas en haut les contributions cumulées du continuum et du combinatoire. 


\section{Ajustement en $\Delta t$ dans la région du signal}

Les paramètres du signal et du continuum sont alors ajustés dans la région du signal en suivant la procédure exposée section 5.3. On ajuste les mêmes paramètres que sur Monte-Carlo, ainsi que le paramètre $\epsilon_{D^{*} \pi}$, défini à l'équation 5.23.

Les résultats sont résumés dans le tableau 5.30 et la figure correspondante est montrée figure 5.27 en échelle linéaire et figure 5.28 en échelle logarithmique. Dans l'analyse, la région du signal correspond aux valeurs de $m_{\text {rec }}$ comprises entre $1,845 \mathrm{GeV} / c^{2}$ et 1,88 $\mathrm{GeV} / c^{2}$. Cependant, afin de réduire la quantité de bruit de fond dans la région du signal, les figures ont été réalisées en réduisant cette région à l'intervalle $1,855 \mathrm{GeV} / c^{2}$ et en faisant la coupure $F<0$ sur la distribution de Fisher. Les fractions des différentes catégories d'événements ont alors été recalculées avec ces coupures.

\begin{tabular}{|c|c|}
\hline \multicolumn{2}{|c|}{$\begin{array}{l}\text { Données, région du signal } \\
\text { Étiquetage par un lepton }\end{array}$} \\
\hline \multicolumn{2}{|c|}{ Signal $D^{*} \pi$} \\
\hline$a D^{*} \pi$ & $-0,042 \pm 0,019$ \\
\hline$c D^{*} \pi$ & $-0,019 \pm 0,022$ \\
\hline$\Delta m_{D^{*} \pi}\left(p s^{-1}\right)$ & $0,518 \pm 0,010$ \\
\hline$\tau_{D^{*} \pi}(p s)$ & $1,450 \pm 0,017$ \\
\hline$\omega_{D^{*} \pi}$ (dir) & $0,010 \pm 0,006$ \\
\hline$\epsilon_{D^{*} \pi}$ (dir) & $0,027 \pm 0,010$ \\
\hline$b_{D^{*} \pi}^{n}(\operatorname{cas})$ & $-0,59 \pm 0,16$ \\
\hline$b_{D^{*} \pi}^{w}(\operatorname{cas})$ & $0,23 \pm 2.01$ \\
\hline$f_{D^{*} \pi}^{n}$ & $0,978 \pm 0,008$ \\
\hline$s_{D^{*} \pi}^{n}$ & $1,08 \pm 0,03$ \\
\hline$s_{D^{*} \pi}^{w}$ & $5,76 \pm 0,28$ \\
\hline \multicolumn{2}{|c|}{ Continuum } \\
\hline$\tau_{q \bar{q}}(p s)$ & $1,26 \pm 0,32$ \\
\hline$\omega_{q \bar{q}}$ & $0,34 \pm 0,01$ \\
\hline$f_{q \bar{q}}^{\delta}$ & $0,81 \pm 0,06$ \\
\hline$b_{q \bar{q}}^{n}$ & $0,03 \pm 0,05$ \\
\hline$b_{q \bar{q}}^{w}$ & $-0,39 \pm 0,24$ \\
\hline$f_{q \bar{q}}^{n}$ & $0,65 \pm 0,12$ \\
\hline$f_{q \bar{q}}^{o}$ & $0,07 \pm 0,01$ \\
\hline$s_{q \bar{q}}^{n}$ & $0,93 \pm 0,07$ \\
\hline$s_{q \bar{q}}^{w}$ & $1,81 \pm 0,28$ \\
\hline
\end{tabular}

TAB. 5.30: Ajustement de la distribution en $\Delta t$ dans la région du signal pour des événements de données, étiquetés par un lepton.

On corrige le temps de vie du biais $+0,007 \pm 0,004$ dû à la paramétrisation de la fonction de résolution (table 5.17, partie 5.5.5) et du biais dû au bruit de fond $B \bar{B}$ $+0,026 \pm 0,013$ (table 5.26, partie 5.6.2). On détermine l'erreur systématique totale en additionnant en quadrature les erreurs systématiques des biais. On obtient $\tau_{D^{*} \pi}=$ 

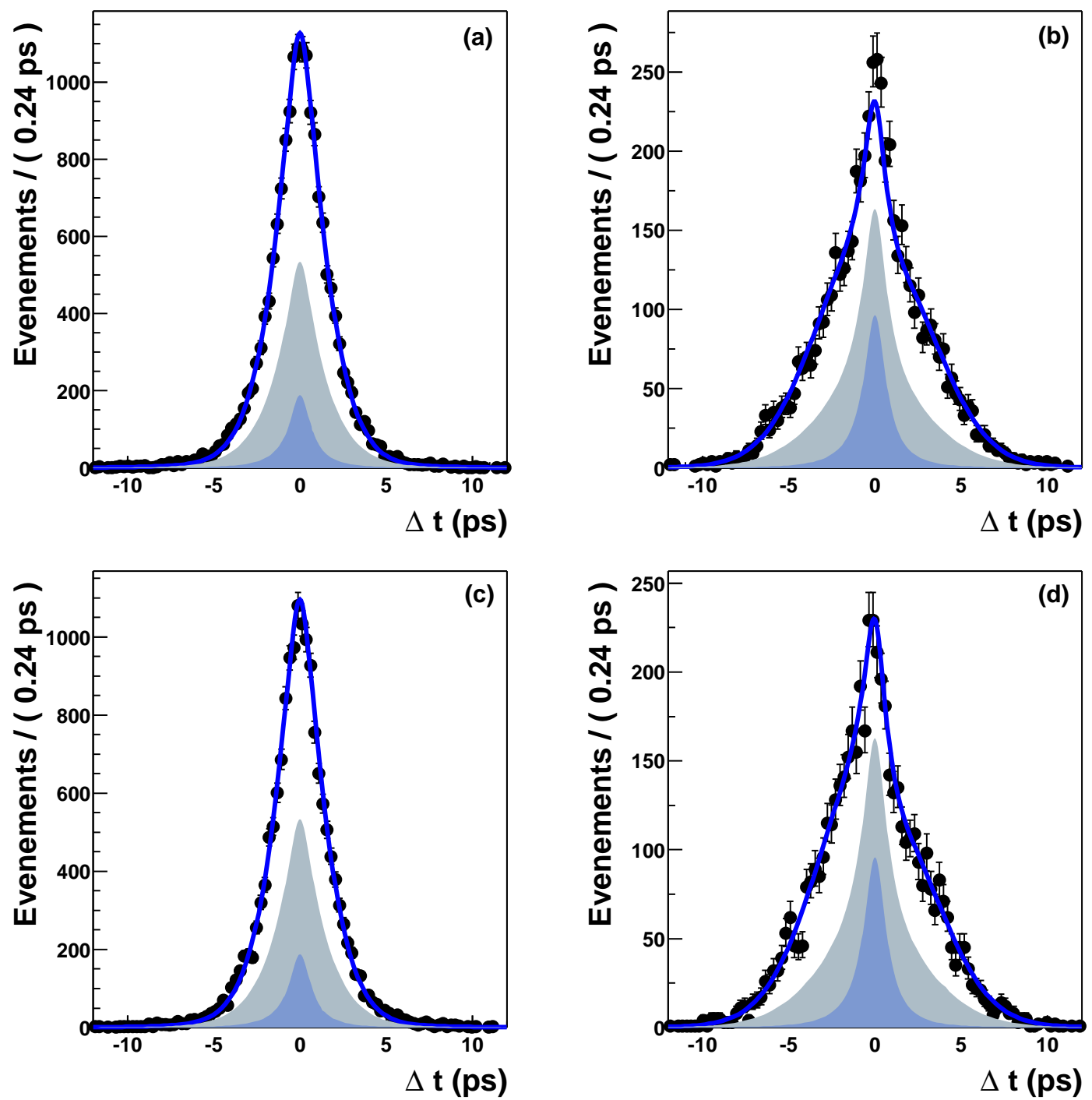

FIG. 5.27: Résultat de l'ajustement sur les données dans la région du signal pour les événements étiquetés par un lepton. Les quatre figures, de gauche à droite et de bas en haut correspondent aux événements non-mélangés étiquetés par un $B^{0}$, aux événements mélangés étiquetés par un $B^{0}$, aux événements non-mélangés étiquetés par un $\bar{B}^{0}$, aux événements mélangés étiquetés par un $\bar{B}^{0}$. Les courbes montrent de bas en haut les contributions cumulées du continuum, du bruit de fond $B \bar{B}$ et du signal $D^{*} \pi$. Afin de réduire le bruit de fond, les coupures $m_{\mathrm{rec}}>1,855$ et $F<0$ ont été appliquées.

1, 484 $\pm 0,013$ (stat.) $\pm 0,014$ (syst.) ps. La valeur du PDG [29] est : $\tau_{B^{0}}=1,536 \pm 0,014$ ps. La valeur mesurée dévie de la valeur du PDG de 2,2 déviations standards.

La valeur de la fréquence de mélange $B^{0} \bar{B}^{0}$ du PDG est $\Delta m_{B}^{0}=0,502 \pm 0,007 \mathrm{ps}^{-1}$. La valeur de $\Delta m_{D^{*} \pi}$ ajustée est bien compatible avec la valeur du PDG : elle dévie d'environ une déviation standard. 

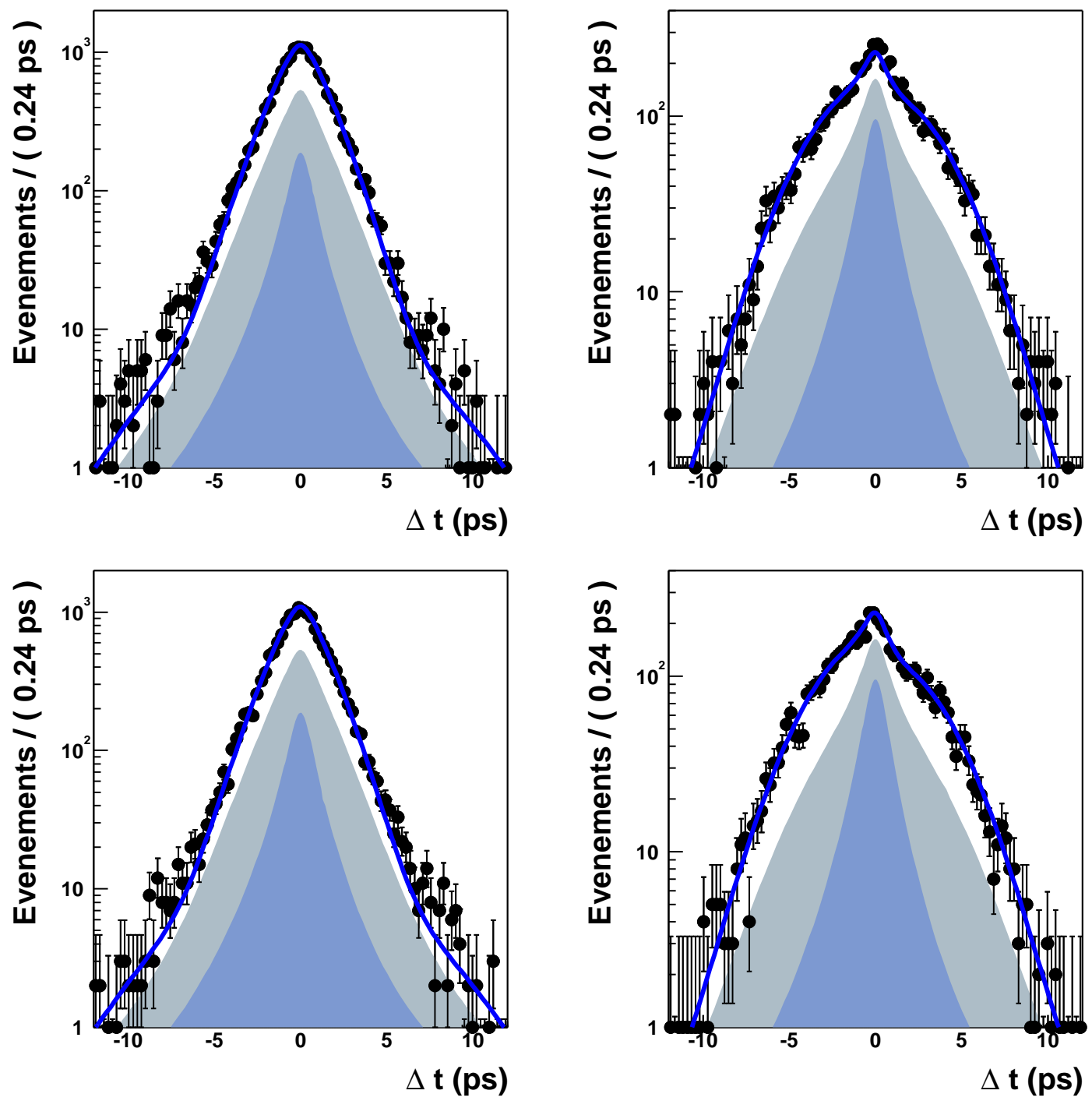

Fig. 5.28: Résultat de l'ajustement sur les données en échelle logarithmique dans la région du signal pour les événements étiquetés par un lepton. Les quatre figures, de gauche à droite et de bas en haut correspondent aux événements non-mélangés étiquetés par un $B^{0}$, aux événements mélangés étiquetés par un $B^{0}$, aux événements non-mélangés étiquetés par un $\bar{B}^{0}$, aux événements mélangés étiquetés par un $\bar{B}^{0}$. Les courbes montrent de bas en haut les contributions cumulées du continuum, du bruit de fond $B \bar{B}$ et du signal $D^{*} \pi$. Afin de réduire le bruit de fond, les coupures $m_{\text {rec }}>1,855$ et $F<0$ ont été appliquées. 
On définit également l'asymétrie de $C P$ dépendante du temps :

$$
\mathcal{A}_{C P}(\Delta t)=\frac{N_{S_{\mathrm{tag}}=1}(\Delta t)-N_{S_{\mathrm{tag}}=-1}(\Delta t)}{N_{S_{\mathrm{tag}}=1}(\Delta t)+N_{S_{\mathrm{tag}}=-1}(\Delta t)}
$$

En l'absence de bruit de fond et de tout effet expérimental, on a $\mathcal{A}_{C P}(\Delta t)=2 r \sin (2 \beta+$ $\gamma) \sin \left(\Delta m_{\Delta t}\right)$. La figure 5.29 représente l'asymétrie de $C P$ dépendante du temps pour les événements étiquetés par un lepton.

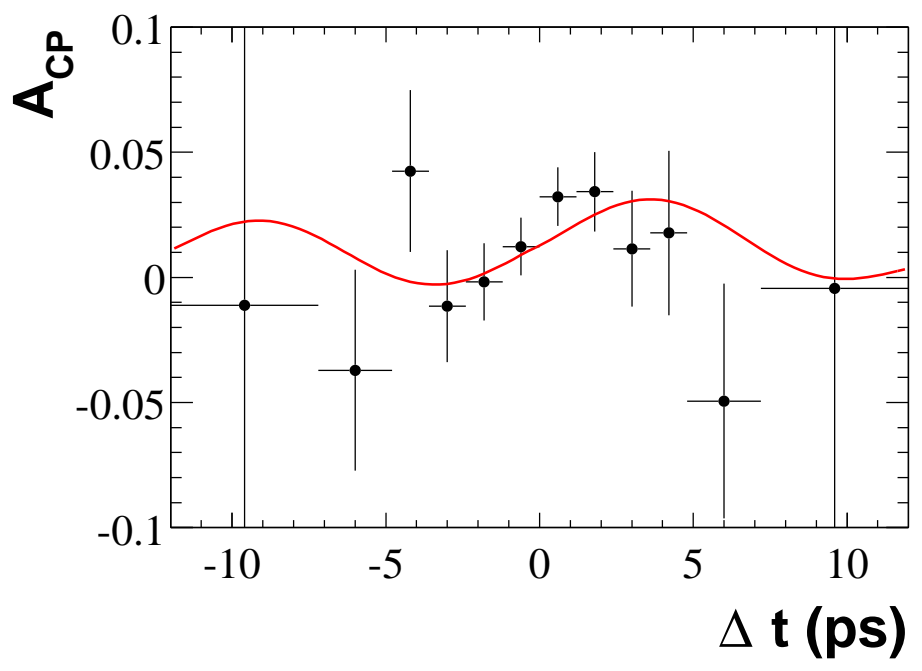

Fig. 5.29: Asymétrie de $C P$ dépendante du temps pour des événements étiquetés par un lepton. La courbe représente la projection de la PDF pour l'asymétrie. Afin de réduire le bruit de fond, les coupures $m_{\mathrm{rec}}>1,855$ et $F<0$ ont été appliquées.

On peut également représenter l'asymétrie due au mélange $B^{0} / \bar{B}^{0}$, définie ainsi :

$$
\mathcal{A}_{\text {mix }}(\Delta t)=\frac{N_{S_{\text {mix }}=1}(\Delta t)-N_{S_{\text {mix }}=-1}(\Delta t)}{N_{S_{\text {mix }}=1}(\Delta t)+N_{S_{\text {mix }}=-1}(\Delta t)} .
$$

En l'absence de bruit de fond et de tout effet expérimental, $\mathcal{A}_{m i x}(\Delta t)=1-\cos (\Delta m \Delta t)$. Cette asymétrie est représentée figure 5.30. 


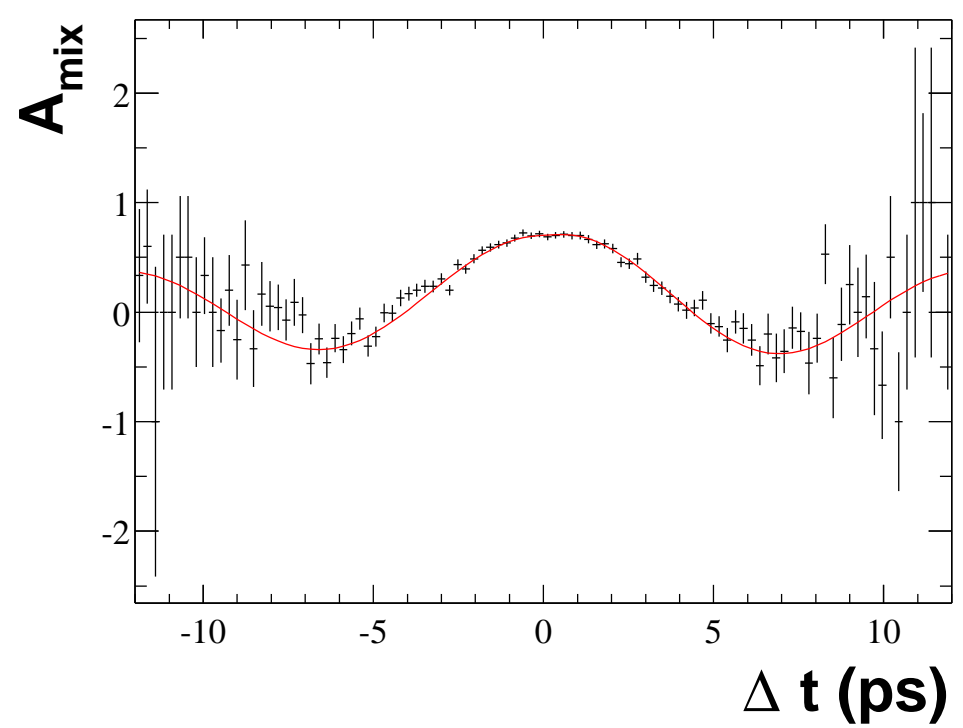

Fig. 5.30: Asymétrie de mélange dépendante du temps pour des événements étiquetés par un lepton. La courbe représente la projection de la PDF pour l'asymétrie. Afin de réduire le bruit de fond, les coupures $m_{\text {rec }}>1,855$ et $F<0$ ont été appliquées. 


\subsubsection{Validation de l'ajustement avec des expériences de Monte- Carlo}

Une étude de Monte-Carlo rapide sert de test de validation de l'analyse, en particulier en ce qui concerne la qualité de l'ajustement et la valeur des erreurs statistiques fournies par MINUIT. De plus, cela peut éventuellement mettre en évidence la présence de biais que l'on n'aurait pas pris en compte.

En pratique, on interprète la PDF avec les paramètres fixés aux valeurs ajustées sur les données, comme une fonction de probabilité pour générer des événements de MonteCarlo rapide avec la même statistique que dans les données. On réalise alors exactement le même ajustement que dans les données sur chaque lot de Monte-carlo rapide ainsi généré. L'étude présentée ici a été réalisée avec 100 expériences de Monte-Carlo rapide.

Différentes quantités nous permettent alors de tester la validité de l'ajustement:

- La quantité $F C N=-\ln (\mathcal{L})$ permet de tester la qualité de l'ajustement. On compare la valeur de $F C N$ renvoyée par l'ajustement sur les données à la distribution de $F C N$ des expériences de Monte-carlo rapide. On voit sur la figure 5.31 que la valeur de FCN des données est compatible avec la distribution de FCN des expériences de Monte-Carlo rapide. Il est possible de quantifier cet accord en calculant un niveau

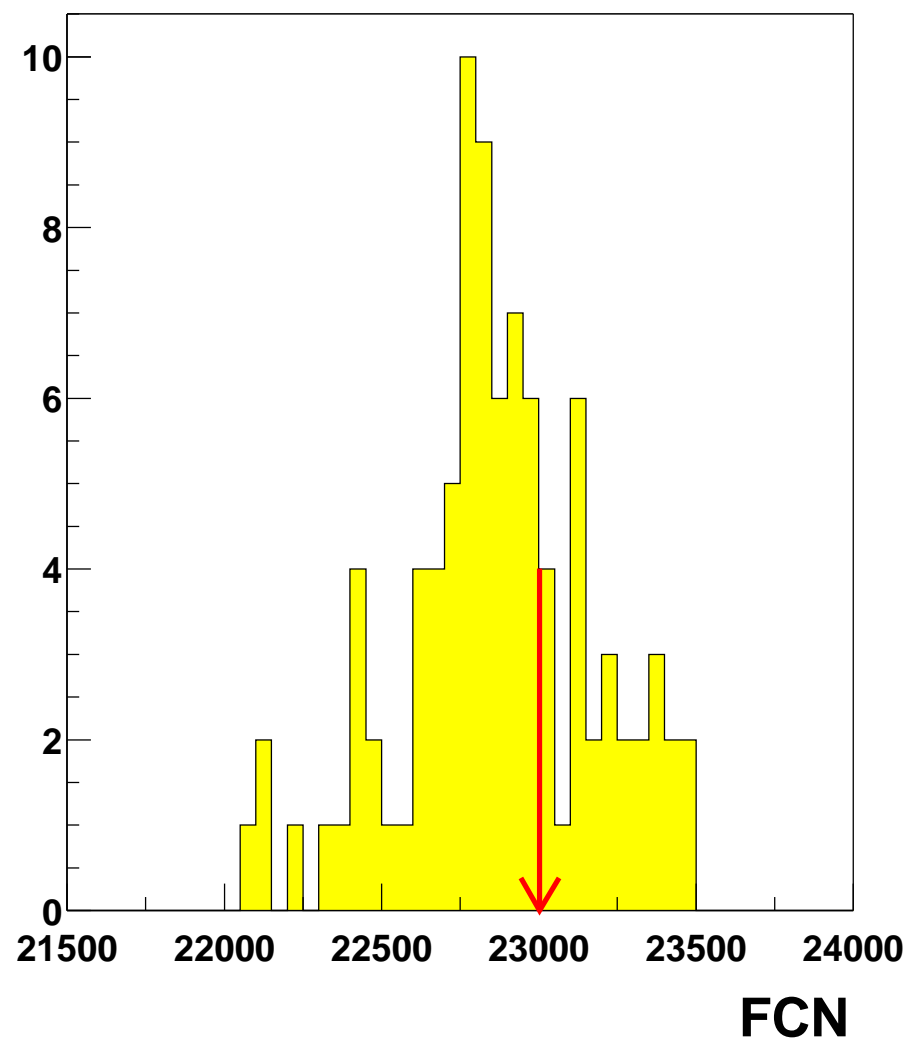

Fig. 5.31: Distribution de FCN des expériences de Monte-Carlo rapide. La flèche représente la valeur de FCN obtenue sur les données. 
de confiance $\mathcal{C}$ qui est la fraction d'expérience de Monte-Carlo rapide qui ont une valeur de FCN plus grande que dans les données:

$$
\mathcal{C}=\frac{N\left(F C N_{M C}>F C N_{\text {donnees }}\right)}{N_{\text {total }}}
$$

On trouve $\mathcal{C}=44 \%$, ce qui valide la qualité de l'ajustement.

- Afin de vérifier l'absence de biais liés à l'ajustement, on compare les valeurs des différents paramètres ajustées sur les données aux distributions de valeurs ajustées sur les expériences de Monte-Carlo. On doit alors s'assurer que les valeurs obtenues sur les données sont bien compatibles avec celles obtenues sur les expériences de Monte-Carlo.

- Pour chaque paramètre libre dans l'ajustement, on compare la valeur de l'erreur statistique obtenue sur les données à la distribution d'erreurs statistiques des expériences de Monte-Carlo rapide. Cela permet de vérifier que la valeur donnée par MINUIT est bien fiable.

Les comparaisons entre les valeurs et erreurs des paramètres ajustés sur les données avec les distributions de valeurs et erreurs des expériences de Monte-Carlo rapide sont montrées figure 5.32 pour les paramètres physiques principaux : $a_{D^{*} \pi}, c_{D^{*} \pi}, \Delta m_{D^{*} \pi}$ et $\tau_{D^{*} \pi}$.

On remarque que les valeurs et erreurs obtenues sur les données sont compatibles avec les distributions des expériences de Monte-Carlo rapide. On en conclue qu'il n'y a pas de biais significatif dans la fonction de vraisemblance et que les erreurs statistiques de l'ajustement sont fiables. 

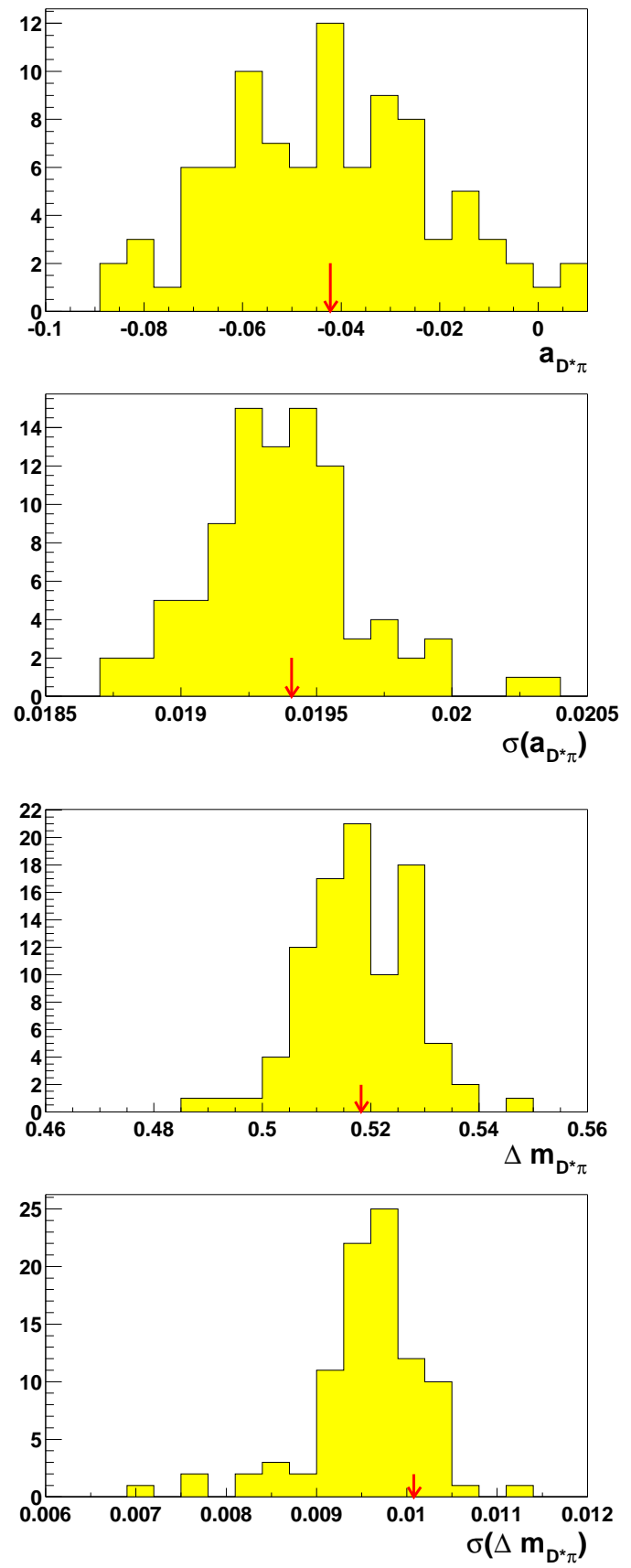
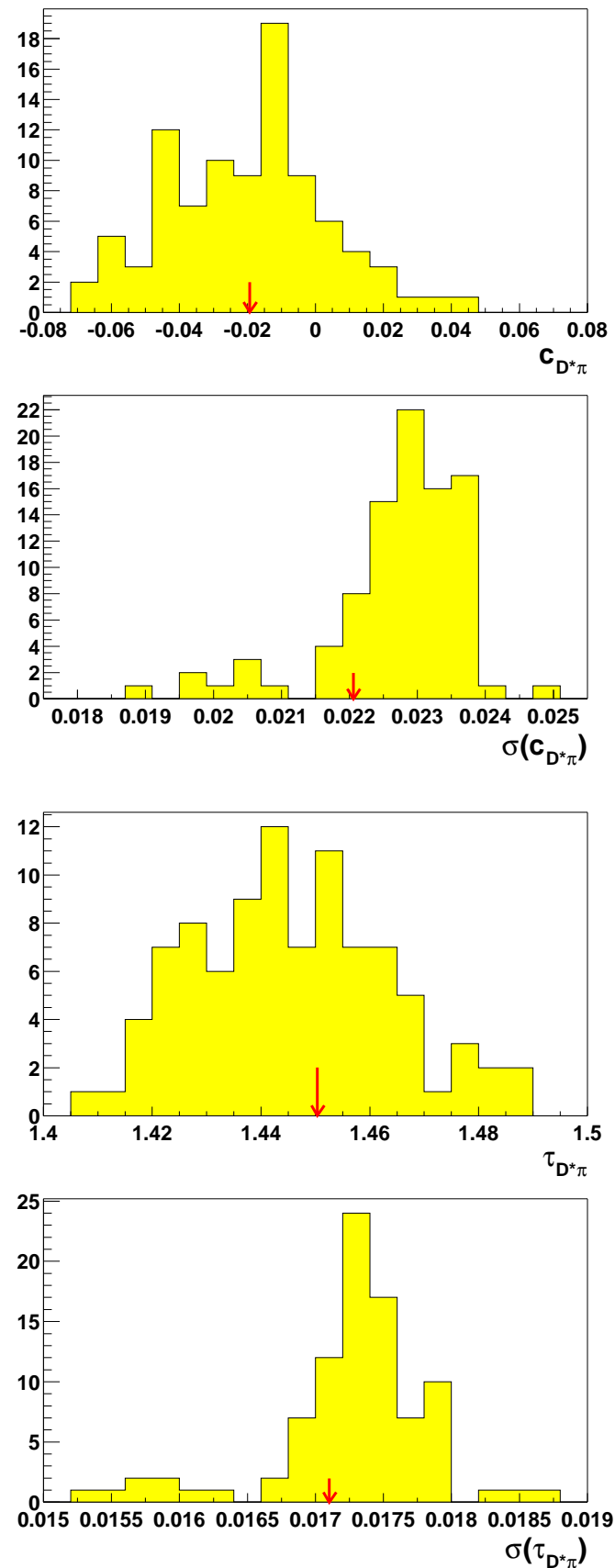

FIG. 5.32: Distributions de la valeur (haut) et de l'erreur (bas) des paramètres $a_{D^{*} \pi}, c_{D^{*} \pi}$, $\Delta m_{D^{*} \pi}$ et $\tau_{D^{*} \pi}$ pour les expériences de Monte-Carlo rapide. Les flèches représentent les valeurs obtenues sur les données, qui sont tout à fait compatibles avec les distributions. 


\subsubsection{Résultats de l'analyse en $\Delta t$ pour les événements étiquetés par un kaon}

\section{Ajustement en $\Delta t$ dans la région latérale en $m_{\text {rec }}$}

La distribution en $\Delta t$ des données est ajustée dans la région latérale $\left(1,81 \mathrm{GeV}<m_{\text {rec }}\right.$ $<1,84 \mathrm{GeV}$ ). Dans cette région, il n'y a, avec une très bonne approximation, que du bruit de fond combinatoire et du continuum. La fraction de continuum pour les données prises à la résonance est calculée en utilisant l'équation 5.15. Les résultats sont résumés dans le tableau 5.31 .

\begin{tabular}{|l|c|}
\hline \multicolumn{2}{|c|}{ Données, région latérale } \\
Étiquetage par un kaon \\
\hline \hline \multicolumn{2}{|c|}{ Combinatoire } \\
\hline$a$ & $-0,0358 \pm 0,0462$ \\
$b$ & $0,0246 \pm 0,0340$ \\
$c$ & $0,0752 \pm 0,0464$ \\
\hline$\Delta m_{c o m b}$ & $0,073 \pm 0,013$ \\
$\tau_{\text {comb }}$ & $1,463 \pm 0,016$ \\
$O_{\text {comb }}$ & $-0,478 \pm 0,052$ \\
$\omega_{\text {comb }}$ & $0,134 \pm 0,026$ \\
\hline$b_{\text {comb }}^{n}$ & $-0,175 \pm 0,034$ \\
$b_{\text {comb }}^{w}$ & $-0,573 \pm 0,101$ \\
$f_{\text {comb }}^{n}$ & $0,752 \pm 0,052$ \\
$f_{\text {comb }}^{o}$ & $0,015 \pm 0,004$ \\
$s_{\text {comb }}^{n}$ & $0,660 \pm 0,078$ \\
$s_{\text {comb }}^{w}$ & $2,182 \pm 0,201$ \\
\hline \multicolumn{2}{|c|}{ Continuum } \\
\hline$f_{\delta}^{q \bar{q}}$ & $0,871 \pm 0,009$ \\
$\tau_{q \bar{q}}$ & $0,809 \pm 0,047$ \\
$\omega_{q \bar{q}}^{\tau}$ & $0,000 \pm 0,002$ \\
$\omega_{q \bar{q}}^{\delta}$ & $0,347 \pm 0,004$ \\
\hline$b_{q \bar{q}}^{n}$ & $0,015 \pm 0,006$ \\
$b_{q \bar{q}}^{w}$ & $0,089 \pm 0,044$ \\
$f_{q \bar{q}}^{n}$ & $0,831 \pm 0,023$ \\
$f_{q \bar{q}}^{o}$ & $0,016 \pm 0,001$ \\
$s_{q \bar{q}}^{q}$ & $1,026 \pm 0,011$ \\
$s_{q \bar{q}}^{w}$ & $1,837 \pm 0,084$ \\
\hline \hline & \\
\hline
\end{tabular}

ТАВ. 5.31: Résultats de l'ajustement des paramètres décrivant le bruit de fond combinatoire et le continuum dans la région latérale en $m_{\text {rec }}$ pour des événements de données, étiquetés par un kaon. 


\section{Ajustement en $\Delta t$ dans la région du signal}

Les paramètres des PDFs décrivant le signal et le continuum sont alors ajustés dans la région du signal en suivant la procédure exposée section 5.3. Les résultats sont résumés dans le tableau 5.32 et les figures correspondantes sont montrées figure 5.33. Les probabilités de Kolmogorov-Smirnov pour les quatre figures sont respectivement, de gauche à droite et de haut en bas, $53 \%, 58 \%, 30 \%$ et $28 \%$. L'ajustement est donc de bonne qualité.

\begin{tabular}{|c|c|}
\hline \multicolumn{2}{|c|}{ Données, région du signal } \\
Étiquetage par un kaon \\
\hline \hline \multicolumn{2}{|c|}{ Signal $D^{*} \pi$} \\
\hline$a$ & $-0,025 \pm 0,020$ \\
$b$ & $-0,004 \pm 0,010$ \\
$c$ & $-0,002 \pm 0,020$ \\
$\Delta m_{D^{*} \pi}$ & $0,4911 \pm 0,0076$ \\
$\Delta \omega_{D^{*} \pi}$ & $-0,0266 \pm 0,0068$ \\
$\tau_{D^{*} \pi}$ & $1,449 \pm 0,011$ \\
$\omega_{D^{*} \pi}$ & $0,230 \pm 0,0035$ \\
$\epsilon_{D^{*} \pi}$ & $-0,0074 \pm 0,0073$ \\
\hline$b_{D^{*} \pi}^{n}$ & $-0,255 \pm 0,013$ \\
$b_{D^{*} \pi}^{w}$ & $-2,070 \pm 0,475$ \\
$f_{D^{*} \pi}^{n}$ & $0,969 \pm 0,007$ \\
$f_{D^{*} \pi}^{o}$ & $0,000 \pm 0,001$ \\
$s_{D^{*} \pi}^{n}$ & $1,029 \pm 0,023$ \\
$s_{D^{*} \pi}^{w}$ & $4.350 \pm 0,401$ \\
\hline \multicolumn{2}{|c|}{ Continuum } \\
\hline$f_{\delta}$ & $0,820 \pm 0,015$ \\
$\tau_{q \bar{q}}$ & $0,707 \pm 0,048$ \\
$\omega_{q \bar{q}}^{\tau}$ & $0,045 \pm 0,022$ \\
$\omega_{q \bar{q}}^{\delta}$ & $0,311 \pm 0,006$ \\
\hline$b_{q \bar{q}}^{n}$ & $0,017 \pm 0,005$ \\
$b_{q \bar{q}}^{w}$ & $-0,043 \pm 0,043$ \\
$f_{q \bar{q}}^{n}$ & $0,858 \pm 0,014$ \\
$f_{q \bar{q}}^{o}$ & $0,018 \pm 0,001$ \\
$s_{q \bar{q}}^{n}$ & $1,064 \pm 0,008$ \\
$s_{q \bar{q}}^{w}$ & $2,267 \pm 0,099$ \\
\hline
\end{tabular}

TAB. 5.32: Résultats de l'ajustement des paramètres décrivant le signal $D^{*} \pi$ et le continuum dans la région du signal pour des événements de données, étiquetés par un kaon.

Le temps de vie corrigé pour les kaons est $\tau_{D^{*} \pi}=1,490 \pm 0,011$ (stat) $\pm 0,020$ (syst), ce qui est compatible avec la valeur du PDG à 1,7 déviations standards. La valeur de la fréquence de mélange $B^{0} \bar{B}^{0}$ du PDG est $\Delta m_{B}^{0}=0,502 \pm 0,007 p s^{-1}$. La valeur de $\Delta m_{D^{*} \pi}$ 
ajustée est bien compatible avec la valeur du PDG : elle dévie d'environ une déviation standard.
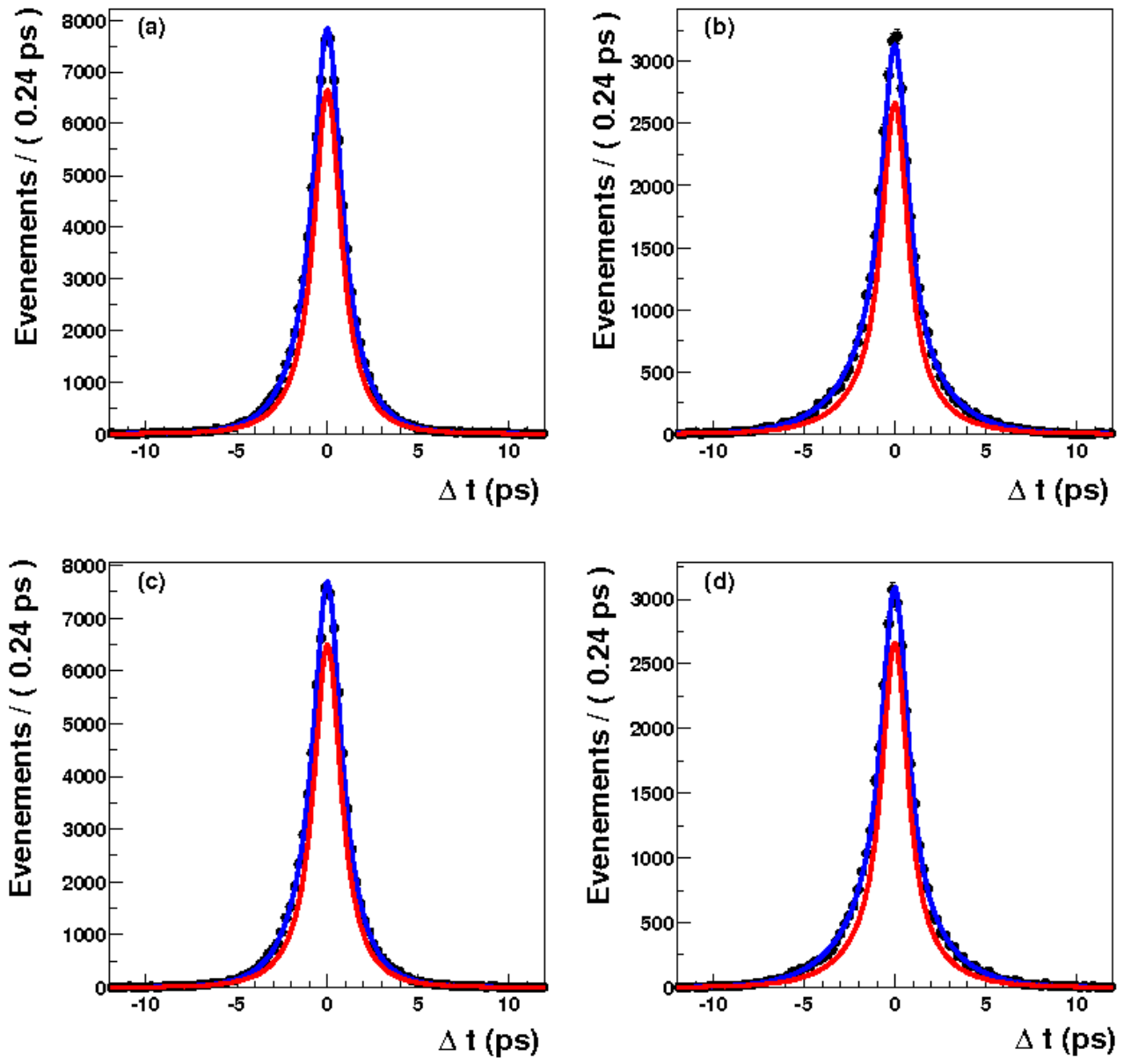

Fig. 5.33: Résultat de l'ajustement sur les données dans la région du signal pour les événements étiquetés par un kaon. Les quatre figures, de gauche à droite et de bas en haut correspondent aux événements non-mélangés étiquetés par un $B^{0}$, aux événements mélangés étiquetés par un $B^{0}$, aux événements non-mélangés étiquetés par un $\bar{B}^{0}$, aux événements mélangés étiquetés par un $\bar{B}^{0}$. La courbe grise correspond aux contributions des bruits de fond et la courbe noire aux contributions cumulées du bruit de fond et du signal. 
Les asymétries de $C P$ et de mélange dépendantes du temps sont représentées figures 5.34 et 5.35 .

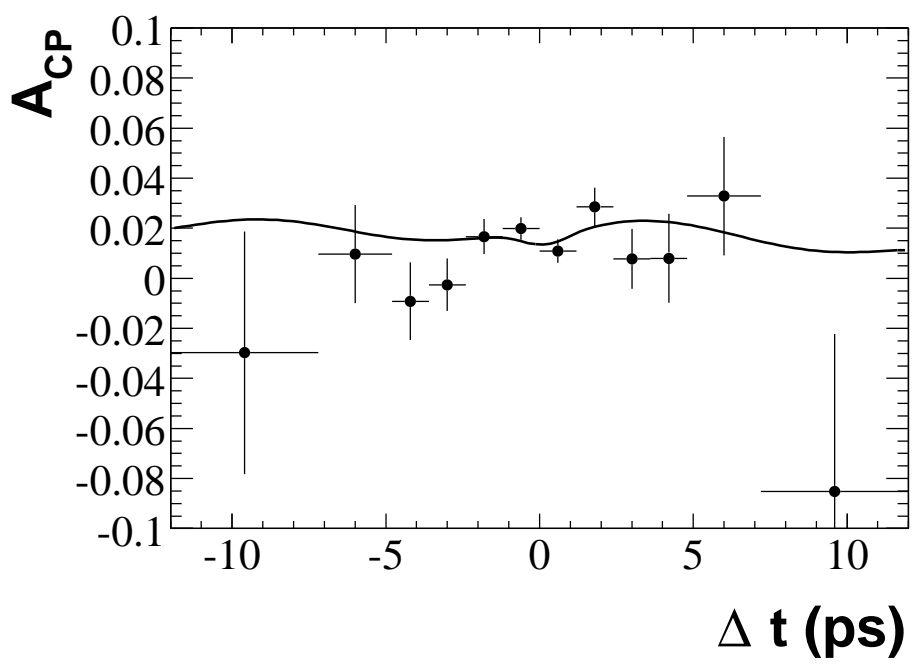

Fig. 5.34: Asymétrie de $C P$ dépendante du temps pour des événements étiquetés par un kaon. La courbe représente la projection de la PDF pour l'asymétrie. Afin de réduire le bruit de fond, les coupures $m_{\text {rec }}>1,855$ et $F<0$ ont été appliquées.

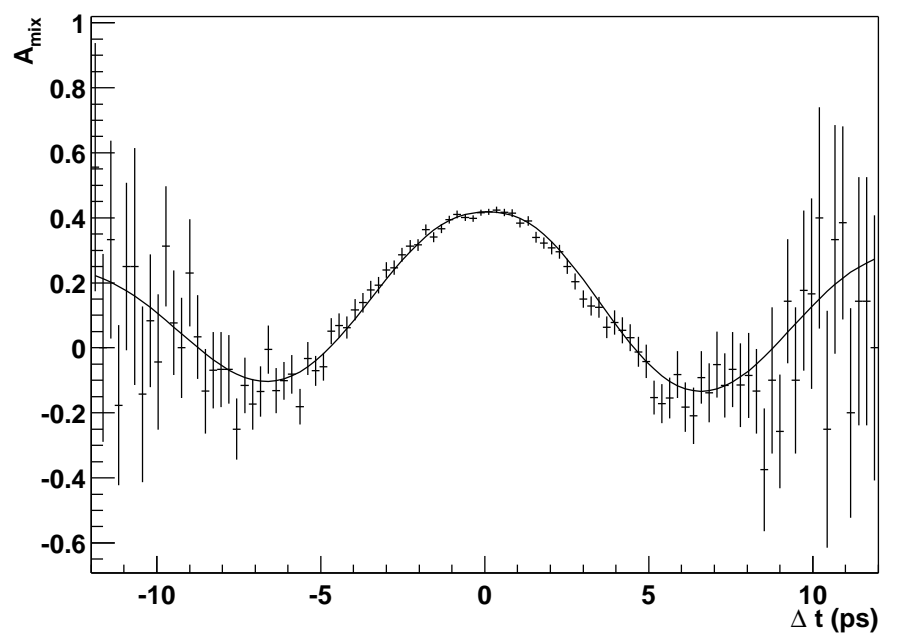

FIG. 5.35: Asymétrie de mélange dépendante du temps pour des événements étiquetés par un kaon. La courbe représente la projection de la PDF pour l'asymétrie. Afin de réduire le bruit de fond, les coupures $m_{\text {rec }}>1,855$ et $F<0$ ont été appliquées. 


\section{8 Étude des erreurs systématiques}

Plusieurs sources de systématiques pouvant biaiser la mesure des paramètres de $C P$ ont été étudiées. Les effets systématiques peuvent provenir de la méthode utilisée (ajustement en quatre étapes, décrit dans la partie 5.3), de la mauvaise connaissance du détecteur, de l'éventuelle présence de violation de $C P$ dans le bruit de fond, du fait que certains paramètres sont fixés dans l'ajustement et de la statistique limitée du Monte-Carlo qui a permis de valider la méthode. Ils sont décrits ci-dessous :

\subsubsection{Systématiques dues à la méthode de l'ajustement en quatre étapes}

\section{Propagation des erreurs statistiques de l'ajustement des variables ciné- matiques :}

Dans l'ajustement final en $\Delta t$ dans la région du signal, on fixe les paramètres des PDFs $\mathcal{F}$ et $\mathcal{M}$ aux valeurs ajustées dans la première étape de l'analyse. Afin de tenir compte de l'incertitude sur cet ajustement, on propage les erreurs statistiques dans l'ajustement final, en tenant compte des corrélations.

L'ajustement des variables cinématiques fournit un vecteur $\vec{p}$ de paramètres, que l'on utilise par la suite dans l'ajustement final ainsi qu'une matrice $V$ des erreurs statistiques. On diagonalise la matrice des erreurs $V$, afin de se placer dans une base où les erreurs ne sont pas corrélées. On appelle $D$ la matrice (diagonale) des erreurs dans cet espace et $T$ la matrice de changement de base. Les matrices $V$ et $D$ sont donc reliées ainsi : $D \equiv T V T^{-1}$. On appelle $\vec{q}$ le vecteur de paramètres dans la base non-corrélée : $\vec{q}=T \vec{p}$. L'erreur associée au paramètre $i$, de valeur $q_{i}$ dans cette base non corrélée est donc $\sqrt{D_{i i}}$.

On définit les vecteurs $\vec{q}^{ \pm, i}$ dont tous les éléments sont égaux à ceux du vecteur $\vec{q}$, à part l'élément $i$ qui est varié de plus ou moins une déviation standard :

$$
\vec{q}^{ \pm, i} \equiv\left(\begin{array}{c}
q_{1} \\
\cdots \\
q_{i} \pm \sqrt{D_{i i}} \\
\cdots \\
q_{N}
\end{array}\right)
$$

On calcule ensuite les vecteurs $\vec{p}^{ \pm, i}$ dans la base de départ (c'est-à-dire la base dans laquelle les paramètres sont corrélés) : $\vec{p}^{ \pm, i}=T^{-1} \vec{q}^{ \pm, i}$. On utilise alors chacun de ces vecteurs de paramètres pour reproduire l'ajustement final et on obtient les paramètres de $C P x_{ \pm, i}$, où $x=a, b$ ou $c$.

L'erreur systématique totale associée à cet effet est alors :

$$
\sigma_{x}^{2}=\sum_{i=1}^{N} \frac{\left(x_{+, i}-x_{0}\right)^{2}+\left(x_{-, i}-x_{0}\right)^{2}}{4},
$$


où $x_{0}$ correspond au résultat de l'ajustement nominal du paramètre $x$.

2. Propagation des erreurs statistiques de l'ajustement dans la région latérale en $m_{\text {rec }}$ :

De la même manière, on propage l'incertitude statistique des paramètres de l'ajustement dans la région latérale et on calcule l'erreur systématique correspondante.

3. Propagation des erreurs statistiques de l'ajustement de $\alpha_{D^{*} \pi}$ et $\rho_{D^{*} \pi}$ :

On propage également les erreurs statistiques de l'ajustement des paramètres $\alpha_{D^{*} \pi}$ et $\rho_{D^{*} \pi}$. Cela consiste simplement à refaire l'ajustement final en faisant varier ces paramètres de plus ou moins une déviation standard. La déviation des paramètres de $C P$ observée par rapport à l'ajustement de référence est alors prise comme erreur systématique.

\subsubsection{Systématiques dues au dispositif expérimental}

N'ayant pas une connaissance parfaite des caractéristiques des faisceau et du détecteur, il est nécessaire d'estimer les erreurs systématiques que peuvent engendrer une mauvaise connaissance du dispositif expérimental sur la mesure des paramètres de $C P$.

\section{Incertitude sur la position moyenne du faisceau}

Il existe des incertitudes sur la position moyenne du point d'interaction des faisceaux, qui sont répercutées sur l'ajustement des vertex des $B$. Afin d'estimer la systématique associée, on a produit un échantillon de signal Monte-Carlo, dans lequel la position moyenne du point d'interaction a été augmentée de $20 \mu m$ dans la direction de l'axe $y$. On calcule alors la déviation des paramètres de CP par rapport à la valeur ajustée avec un échantillon de signal Monte-Carlo standard. L'erreur systématique associée à l'incertitude sur la position moyenne du faisceau est alors égale à cette déviation.

\section{2. Échelle selon l'axe $z$}

On estime de la même manière l'erreur systématique associée à l'incertitude sur les mesures de distances dans la direction longitudinale (selon l'axe $z$ des faisceaux) en utilisant un échantillon de Monte-Carlo généré avec une variation de 0,6\% de l'échelle de distance selon l'axe $z$.

\section{Alignement du détecteur de vertex}

L'alignement du détecteur de vertex est primordial dans cette analyse. En effet, on étudie le phénomène de violation de $C P$ dépendant du temps et nécessitant donc d'une très bonne connaissance de la position des vertex des $B$, qui est principalement fournie par le détecteur de vertex. Afin d'évaluer la systématique associée, on utilise une procédure standard de $B A B A R$. Cela consiste à générer des échantillons 
de signal Monte-Carlo avec différents fichiers de paramètres d'alignement. Les variations des paramètres entre ces différents ensembles paramétrisent la connaissance imparfaite de la position des tuiles du détecteur de vertex ainsi que certains biais connus. L'idée est de relier tout effet de mauvais alignement à des distorsions comprises et bien définies du détecteur. On calcule alors la déviation des paramètres de $C P$ par rapport à la valeur ajustée sur un échantillon Monte-Carlo de référence. En pratique, on dispose de quatre fichiers de paramètres d'alignement qui modélisent la dépendance temporelle de l'alignement du détecteur de vertex et d'un fichier de paramètres qui modélise un biais observé qui se traduit par un décalage dépendant de la position $z$ des couches du détecteur de vertex. La systématique totale due au mauvais alignment du détecteur de vertex pour chaque paramètre de $C P$ est alors la somme quadratique de la plus grande déviation observée par rapport à la valeur de référence en utilisant un des quatre fichiers modélisant la dépendance temporelle et de la déviation observée avec le dernier fichier.

\subsubsection{Systématiques dues à l'éventuelle présence de violation de $C P$ dans le bruit de fond}

Dans l'analyse, on a supposé qu'il n'y avait pas de violation de $C P$ dans le bruit de fond. En effet, les paramètres $(a, b, c)_{D^{*} \rho},(a, b, c)_{\text {comb }}$ et $(a, b, c)_{\text {peak }}$ sont fixés à 0 dans l'ajustement. Afin d'estimer la systématique associée à l'éventuelle présence de violation de $C P$ dans le bruit de fond, on répète l'analyse en supposant qu'il y a violation de $C P$ dans le bruit de fond.

Il paraît raisonnable de supposer que le rapport $r^{*}$ du bruit de fond est du même ordre de grandeur que celui du signal. En réalité, on s'attend même à ce qu'il soit plus faible dans le cas du bruit de fond qui pique ou du combinatoire, car ils contiennent différents types d'événements avec a priori des phases fortes différentes. On suppose $r_{\text {bruitdefond }}^{*}=0,02$ pour estimer la systématique, ce qui est donc très conservatif. On suppose de plus une violation de $C P$ maximale dans le bruit de fond, avec $\sin (2 \beta+\gamma)=1$. On étudie l'effet de la présence de violation de $C P$ dans le bruit de fond pour chaque type de bruit de fond séparément.

\section{Événements étiquetés par un lepton}

Pour les événements étiquetés par un lepton, nous avons considéré quatre couples de paramètres $(a, c)$ pour chaque type de bruit de fond, correspondant aux valeurs de phases fortes relatives $\delta=0, \pi / 2, \pi, 3 \pi / 2$ :

$-a=0,04$ et $c=0$

$-a=-0,04$ et $c=0$

$-a=0$ et $c=-0,04$

$-a=0$ et $c=-0,04$

Les variations de l'ajustement des paramètres $a_{D^{*} \pi}$ et $c_{D^{*} \pi}$ sont résumées dans les tableaux 5.33, 5.34 et 5.35 . 


\begin{tabular}{|l|c|c|}
\hline$D^{*} \rho$ & $\Delta a$ & $\Delta c$ \\
\hline$a=0,04 ; c=0$ & $-0,0053$ & negl. \\
$a=-0,04 ; c=0$ & 0,0052 & negl. \\
$a=0 ; c=0,04$ & negl. & $-0,0049$ \\
$a=0 ; c=-0,04$ & negl. & 0,0052 \\
\hline
\end{tabular}

TAB. 5.33: Variation des paramètres de $C P$ du signal $D^{*} \pi$ étiqueté par un lepton quand on varie les paramètres de $C P$ du bruit de fond $D^{*} \rho$

\begin{tabular}{|l|c|c|}
\hline combinatoire & $\Delta a$ & $\Delta c$ \\
\hline$a=0,04 ; c=0$ & $-0,0025$ & negl. \\
$a=-0,04 ; c=0$ & 0,0025 & negl. \\
$a=0 ; c=0,04$ & negl. & $-0,0022$ \\
$a=0 ; c=-0,04$ & negl. & 0,0023 \\
\hline
\end{tabular}

TAB. 5.34: Variation des paramètres de $C P$ du signal $D^{*} \pi$ étiqueté par un lepton quand on varie les paramètres de $C P$ du bruit de fond combinatoire

\begin{tabular}{|l|c|c|}
\hline bruit de fond qui pique & $\Delta a$ & $\Delta c$ \\
\hline$a=0,04 ; c=0$ & $-0,0036$ & negl. \\
$a=-0,04 ; c=0$ & 0,0037 & negl. \\
$a=0 ; c=0,04$ & negl. & $-0,0034$ \\
$a=0 ; c=-0,04$ & negl. & 0,0038 \\
\hline
\end{tabular}

TAB. 5.35: Variation des paramètres de $C P$ du signal $D^{*} \pi$ étiqueté par un lepton quand on varie les paramètres de $C P$ du bruit de fond qui pique

\section{Événements étiquetés par un kaon}

De manière similaire, on estime l'erreur systématique due à l'éventuelle présence de violation de $C P$ dans le bruit de fond pour les événements étiquetés par un kaon. Cette foisci, on doit également étudier la systématique sur le paramètre $b_{D^{*} \pi}$. Les valeurs utilisées pour étudier les systématiques et les déviations sur les paramètres de $C P$ sont résumées dans les tableaux 5.36, 5.37 et 5.38.

\section{Systématique totale due à la violation de $C P$ dans le bruit de fond}

Pour chaque type de bruit de fond, on prend la plus grande déviation observée sur les paramètres comme erreur systématique. L'erreur systématique totale est alors la somme linéaire des erreurs systématiques pour chaque type de bruit de fond. 


\begin{tabular}{|l|c|c|c|}
\hline$C P$ in $D^{*} \rho$ & $\Delta a$ & $\Delta b$ & $\Delta c$ \\
\hline$a=-0,04, b=0,04, c=0,04$ & 0,0057 & $-0,0058$ & $-0,0058$ \\
$a=0,04, b=0,04, c=-0,04$ & $-0,0056$ & $-0,0054$ & 0,0057 \\
$a=0,04, b=-0,04, c=0,04$ & $-0,0056$ & 0,0055 & $-0,0055$ \\
$a=0,0, b=0,04, c=0$ & 0,0 & $-0,0056$ & $-0,0001$ \\
$a=0,04, b=0, c=0,04$ & $-0,0056$ & $-0,0001$ & $-0,0055$ \\
$a=0,0, b=0, c=0,04$ & 0,0 & $-0,0001$ & $-0,0057$ \\
$a=0,04, b=0,04, c=0$ & $-0,0056$ & $-0,0056$ & 0,0001 \\
\hline
\end{tabular}

TAB. 5.36: Variation des paramètres de $C P$ du signal $D^{*} \pi$ étiqueté par un kaon quand on varie les paramètres de $C P$ du bruit de fond $D^{*} \rho$

\begin{tabular}{|l|c|c|c|}
\hline combinatoire & $\Delta a$ & $\Delta b$ & $\Delta c$ \\
\hline $\mathrm{a}=0,04, \mathrm{~b}=0,04, \mathrm{c}=-0,04$ & $-0,0080$ & $-0,0054$ & 0,0078 \\
$\mathrm{a}=0,0, \mathrm{~b}=0,04, \mathrm{c}=0,04$ & $-0,0004$ & $-0,0056$ & $-0,0072$ \\
$\mathrm{a}=0,04, \mathrm{~b}=0,04, \mathrm{c}=0,0$ & $-0,0080$ & $-0,0056$ & 0,0004 \\
$\mathrm{a}=0,04, \mathrm{~b}=0,0, \mathrm{c}=0,04$ & $-0,0074$ & $-0,0002$ & $-0,0055$ \\
\hline
\end{tabular}

TAB. 5.37: Variation des paramètres de $C P$ du signal $D^{*} \pi$ étiqueté par un kaon quand on varie les paramètres de $C P$ du bruit de fond combinatoire

\begin{tabular}{|c|c|c|c|}
\hline bruit de fond qui pique & $\Delta a$ & $\Delta b$ & $\Delta c$ \\
\hline $\mathrm{a}=-0,04, \mathrm{~b}=0,04, \mathrm{c}=0,04$ & 0,0018 & $-0,0013$ & $-0,0019$ \\
$\mathrm{a}=0,04, \mathrm{~b}=0,04, \mathrm{c}=-0,04$ & $-0,0020$ & $-0,0014$ & 0,0020 \\
\hline
\end{tabular}

TAB. 5.38: Variation des paramètres de $C P$ du signal $D^{*} \pi$ étiqueté par un kaon quand on varie les paramètres de $C P$ du bruit de fond qui pique

\subsubsection{Autres sources d'erreurs systématiques}

1. On évalue la systématique due au fait que l'on ajuste les paramètres du bruit de fond combinatoire dans la région latérale et non dans la région du signal en comparant les résultats de l'ajustement sur Monte-Carlo avec le combinatoire ajusté dans la région latérale et dans la région du signal. La différence entre les paramètres de $C P$ ajustée est prise comme systématique.

2. La fraction de $D^{*} \pi$ par rapport à l'ensemble $D^{*} \pi / D^{*} \rho$ est fixée à une valeur déterminée de la façon décrite dans la partie 5.4.3. Afin d'estimer la systématique correspondante, on refait l'ajustement en $\Delta t$ dans la région du signal en variant cette fraction de plus ou moins une déviation standard. 


\subsubsection{Résumé des erreurs systématiques}

Toutes les erreurs systématiques évoquées plus haut sont résumées dans le tableau 5.39 .

\begin{tabular}{|l|c|c|c|c|c|}
\hline Source & \multicolumn{5}{|c|}{ Krreur $\left(\times 10^{-2}\right)$} \\
\hline Étiquetage & \multicolumn{2}{|c|}{ Lepton } & \multicolumn{3}{|c|}{ Kaon } \\
\hline & $a_{D^{*} \pi}^{L}$ & $c_{D^{*} \pi}^{L}$ & $a_{D^{*} \pi}^{K}$ & $b_{D^{*} \pi}^{K}$ & $c_{D^{*} \pi}^{K}$ \\
\hline 1. Ajustement cinématique & 0,04 & 0,04 & 0,10 & 0,04 & 0,04 \\
2. Statistique dans la bande latérale & 0,08 & 0,08 & 0,40 & 0,12 & 0,44 \\
3. $\alpha$ & 0,02 & 0,02 & 0,02 & negl. & negl. \\
4. $\rho$ & 0,02 & 0,02 & 0,02 & negl. & negl. \\
\hline 5. Statistique du Monte-Carlo & 0,6 & 1,2 & 0,8 & 0,4 & 0,9 \\
\hline 6. Position du faisceau & 0,10 & 0,10 & 0,07 & 0,13 & 0,06 \\
7. Échelle selon $z$ du détecteur & 0,03 & 0,03 & 0,02 & negl. & 0,03 \\
8. Alignement du détecteur & 0,4 & 0,8 & 0,41 & 0,14 & 0,74 \\
\hline 9. Violation de $C P$ dans le combinatoire & 0,25 & 0,22 & 0,80 & 0,56 & 0,72 \\
10, Violation de $C P$ dans le bruit de fond qui pique & 0,36 & 0,38 & 0,29 & 0,17 & 0,27 \\
11. Violation de $C P$ dans $D^{*} \rho$ & 0,53 & 0,52 & 0,57 & 0,58 & 0,58 \\
\hline 12. Bruit de fond qui pique & 0,21 & 0,31 & 0,21 & 0,41 & 0,31 \\
13. Signal region/sideband difference & 0,0003 & 0,002 & 0,04 & 0,03 & 0,05 \\
14. $\mathcal{B}\left(B \rightarrow D^{* \mp} \rho^{ \pm}\right)$ & 0,17 & 0,33 & 0,17 & 0,22 & 0,33 \\
\hline Erreur systématique totale & 0,99 & 1,22 & 1,36 & 1,04 & 1,52 \\
\hline Erreur statistique & 1,9 & 2,2 & 2,0 & 1,0 & 2,0 \\
\hline
\end{tabular}

TAB. 5.39: Résumé des erreurs systématiques pour la mesure des paramètres de $C P$

Les systématiques dominantes sont dues à notre méconnaissance de l'amplitude de la violation de $C P$ dans les bruits de fond. Nous avons cependant adopté une approche très conservative pour estimer ces erreurs. Il sera possible dans l'avenir de réduire ces systématiques, par exemple en mesurant la violation de $C P$ dnas les désintégrations $B^{0} \rightarrow$ $D^{*-} \rho^{+}$. Bien que les erreurs systématiques ne soient pas négligeables, cette analyse reste dominée par la statistique.

\subsection{Combinaison des résultats}

Les valeurs et les erreurs statistiques et systématiques des paramètres de $C P$ mesurées sur les données pour les événements étiquetés par un lepton et par un kaon sont résumées ci-dessous. L'indice ${ }^{L}\left({ }^{K}\right)$ se réfère aux résultats des événements étiquetés par un lepton (kaon). La première erreur correspond à l'erreur statistique et la deuxième erreur à l'erreur systématique. 


$$
\begin{aligned}
& a_{D^{*} \pi}^{L}=-0,042 \pm 0,019 \pm 0,010, \\
& c_{D^{*} \pi}^{L}=-0,019 \pm 0,022 \pm 0,019, \\
& a_{D^{*} \pi}^{K}=-0,025 \pm 0,020 \pm 0,015, \\
& b_{D^{*} \pi}^{K}=-0,004 \pm 0,010 \pm 0,011, \\
& c_{D^{*} \pi}^{K}=-0,002 \pm 0,020 \pm 0,016 .
\end{aligned}
$$

La valeur attendue pour $a_{D^{*} \pi}$ est de l'ordre de $\pm 0,30$ et la valeur attendue pour $b_{D^{*} \pi}$ et $c_{D^{*} \pi}$ est de l'ordre de 0 (voir partie 4.2.4). Les valeurs mesurées sont donc compatibles avec les valeurs attendues.

Les mesures sur les événements étiquetés par un lepton ou par un kaon ont été réalisées sur des échantillons entièrement indépendants. La corrélation statistique entre les paramètres des événements étiquetés par un lepton et les paramètres des événements étiquetés par un kaon est donc nulle. Il existe cependant des corrélations statistiques très faibles entre les paramètres de chaque catégorie, de l'ordre du \%.

Afin d'être le plus conservatif possible, on considère que les erreurs systématiques relatives au détecteur et à l'amplitude inconnue de la violation de $C P$ dans le bruit de fond sont corrélées à $100 \%$ entre les cinq paramètres de $C P$ mesurés. Les autres erreurs systématiques sont considérées comme étant non-corrélées. Les différentes erreurs sont résumées dans le tableau 5.40 .

\begin{tabular}{|l|c|c|c|c|c|}
\cline { 2 - 6 } \multicolumn{1}{c|}{} & \multicolumn{5}{c|}{ Erreur $\left(\times 10^{-2}\right)$} \\
\cline { 2 - 6 } \multicolumn{1}{c|}{} & $a_{D^{*} \pi}^{L}$ & $c_{D^{*} \pi}^{L}$ & $a_{D^{*} \pi}^{K}$ & $b_{D^{*} \pi}^{K}$ & $c_{D^{*} \pi}^{K}$ \\
\hline Erreur statistique & 1,9 & 2,2 & 2,0 & 1,0 & 2,0 \\
\hline Erreur systématique corrélée & 0,74 & 0,88 & 1,05 & 0,84 & 1,05 \\
\hline Erreur systématique non corrélée & 0,66 & 0,84 & 0,81 & 0,62 & 1,06 \\
\hline
\end{tabular}

TAB. 5.40: Résumé des erreurs pour la mesure des paramètres de $C P$

Le seul paramètre dont on peut combiner les mesures des deux catégories est le paramètres $a_{D^{*} \pi}$, car c'est le seul paramètre dont la signification physique soit identique entre les deux catégories. En effet, les paramètres $b_{D^{*} \pi}^{K}$ et $c_{D^{*} \pi}^{K}$ tiennent compte de la possibilité de l'existence de violation de $C P$ du côté de $B_{\text {tag }}$ pour les événements étiquetés par un kaon. Cet effet n'intervenant pas dans le cas des événements étiquetés par un lepton, on ne peut pas combiner la mesure de $c_{D^{*} \pi}$.

En tenant compte des corrélations, on obtient la mesure combinée de $a_{D^{*} \pi}$ suivante :

$$
a_{D^{*} \pi}=-0,034 \pm 0,014 \pm 0,009 .
$$

Cette valeur est totalement compatible avec la valeur attendue qui est $\pm 0,027$ (voir partie 4.2.4). Les paramètres de $C P$ mesurés peuvent être interprétés afin de mettre une limite sur $|\sin (2 \beta+\gamma)|$ en utilisant une approche fréquentiste exposée dans le chapitre 6 . 


\section{6 \\ Interprétation des résultats}

Ce chapitre décrit l'interprétation statistique des résultats présentés dans cette thèse. La méthode exposée ici est basée sur une approche fréquentiste, inspirée de la méthode proposée par Feldman et Cousins [86]. Dans un premier temps, nous allons rappeler la procédure à suivre dans le cas gaussien classique pour déterminer un intervalle de confiance. Ensuite, nous allons démontrer que cette procédure peut conduire à de mauvais intervalles de confiance dans certains cas bien précis et en particulier lorsque l'on dispose d'une mesure proche de la limite physique, ce qui est le cas dans notre analyse, comme on le verra dans la suite. La méthode originale de Feldman-Cousins sera alors décrite, puis on montrera de quelle façon cette méthode a été adaptée dans l'interprétation de notre mesure.

\subsection{Intervalles de confiance classiques}

Cette partie expose la méthode classique pour déterminer un intervalle de confiance, proposée par Neyman en 1937 [87].

\subsubsection{Principe de la méthode de Neyman}

Supposons que l'on mesure une observable $x$ dépendant d'un paramètre physique $m$, dont la vraie valeur (inconnue) est notée $m_{t}$. Remarquons que l'observable que l'on mesure peut être directement égale au paramètre physique $m$ qui nous intéresse $(x(m)=m)$, mais ce n'est pas forcément le cas.

La méthode de Neyman consiste à déterminer, pour chaque valeur de $m_{t}$, un intervalle $\left(x_{1}, x_{2}\right)$ avec un niveau de confiance $\alpha$, tel que la probabilité que la mesure $x_{0}$ soit comprise dans cet intervalle est égale à $\alpha$. On peut alors représenter ces intervalles de confiance pour toute valeur de $m_{t}$, comme le montre la figure 6.1. A ce niveau-là, on n'a pas encore utilisé la mesure expérimentale.

On trace alors une ligne verticale à la valeur mesurée $x_{0}$. L'intervalle avec un niveau de confiance $\alpha$ correspondant à cette mesure est alors l'ensemble des valeurs $m_{t}$ dont la ligne verticale coupe l'intervalle de confiance. 


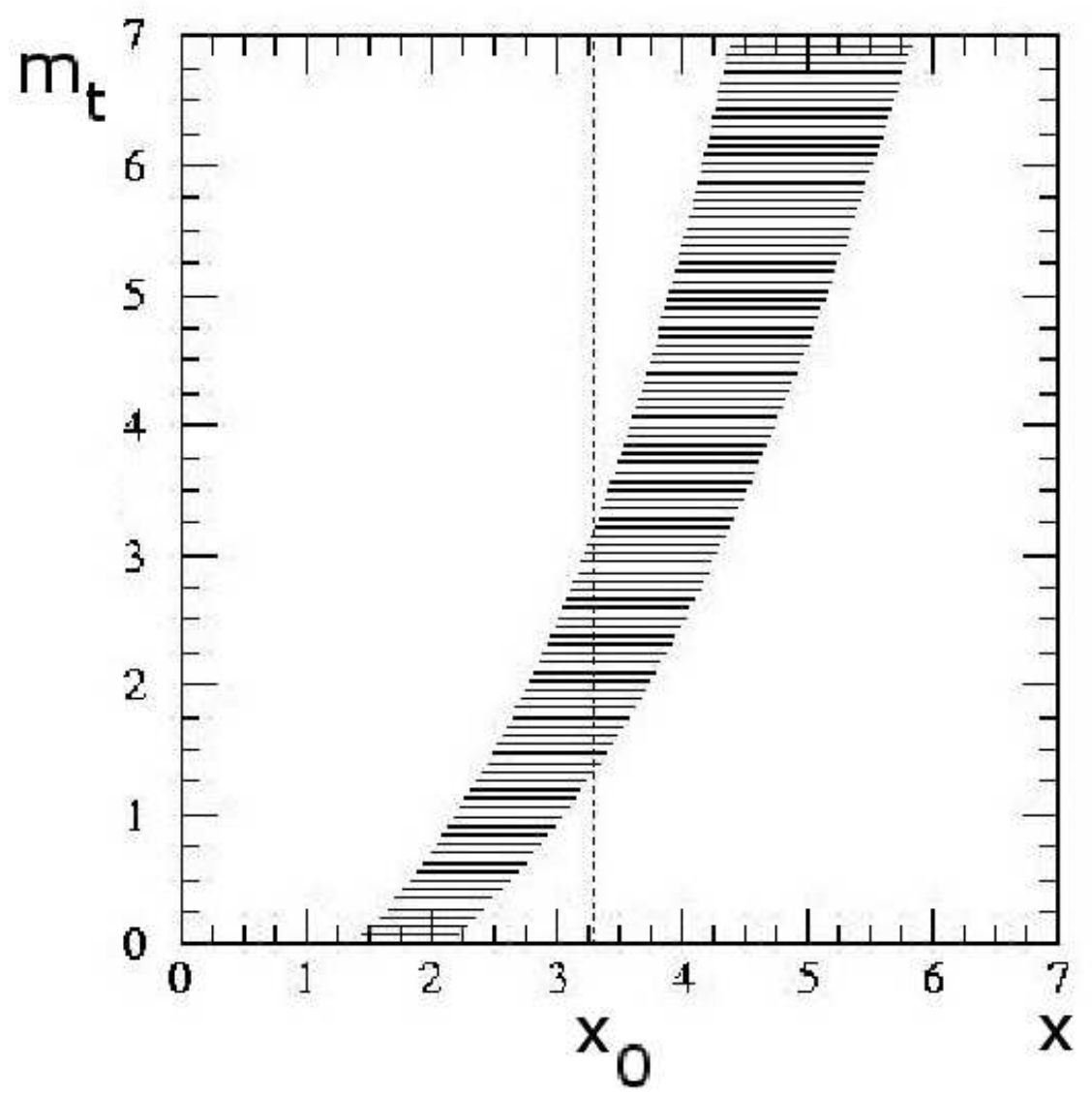

FIG. 6.1: Construction de Neyman pour déterminer un niveau de confiance. Les traits horizontaux représentent les intervalles de confiance pour toute valeur de $m_{t}$. La ligne verticale représente la valeur mesurée $x_{0}$.

\subsubsection{Cas gaussien non borné}

Dans cette partie, on se place dans le cas simple où l'observable mesurée est directement égale au paramètre $m$. La probabilité de mesurer $m$ sachant que la vraie valeur de $m$ est $m_{t}$ suit alors une loi gaussienne [88] :

$$
P\left(m \mid m_{t}\right)=N\left(m_{t}, \sigma_{m}\right)=\frac{1}{\sqrt{\left(2 \pi \sigma_{m}^{2}\right)}} e^{-\left(m-m_{t}\right)^{2} / 2 \sigma_{m}^{2}},
$$

où $\sigma_{m}$ représente l'erreur sur la mesure. On appelle $m_{0}$ la valeur mesurée de $m$ et on cherche à construire un intervalle de confiance $\left(m_{1}, m_{2}\right)$ avec un niveau de confiance $\alpha$. Ici, on prend $\alpha=68 \%$. Supposons que l'on réalise un grand nombre de mesures et que l'on détermine un intervalle à $68 \%$ de niveau de confiance pour chacune de ces mesures, alors $68 \%$ de ces intervalles contiennent la vraie valeur $m_{t}$. Cette définition fréquentiste est à opposer à l'approche bayesienne qui stipule que $m_{t}$ est compris dans l'intervalle à $68 \%$ de niveau de confiance.

De façon classique, on construit un intervalle à $68 \%$ de niveau de confiance, en 
déterminant $m_{1}<m_{0}$ tel que $16 \%$ de l'aire sous $N\left(m_{1}, \sigma_{m}\right)$ corresponde à des valeurs de $m$ supérieures à $m_{0}$ et $m_{2}>m_{0}$ tel que $16 \%$ de l'aire sous $N\left(m_{2}, \sigma_{m}\right)$ corresponde à des valeurs de $m$ inférieures à $m_{0}$. On trouve alors $m_{1}=m_{0}+\sigma_{m}$ et $m_{2}=m_{0}-\sigma_{m}$. Par exemple, si $m_{0}=5$ et $\sigma_{m}=1$, on trouve $m_{1}=4$ et $m_{2}=6$ (voir figure 6.2 ).
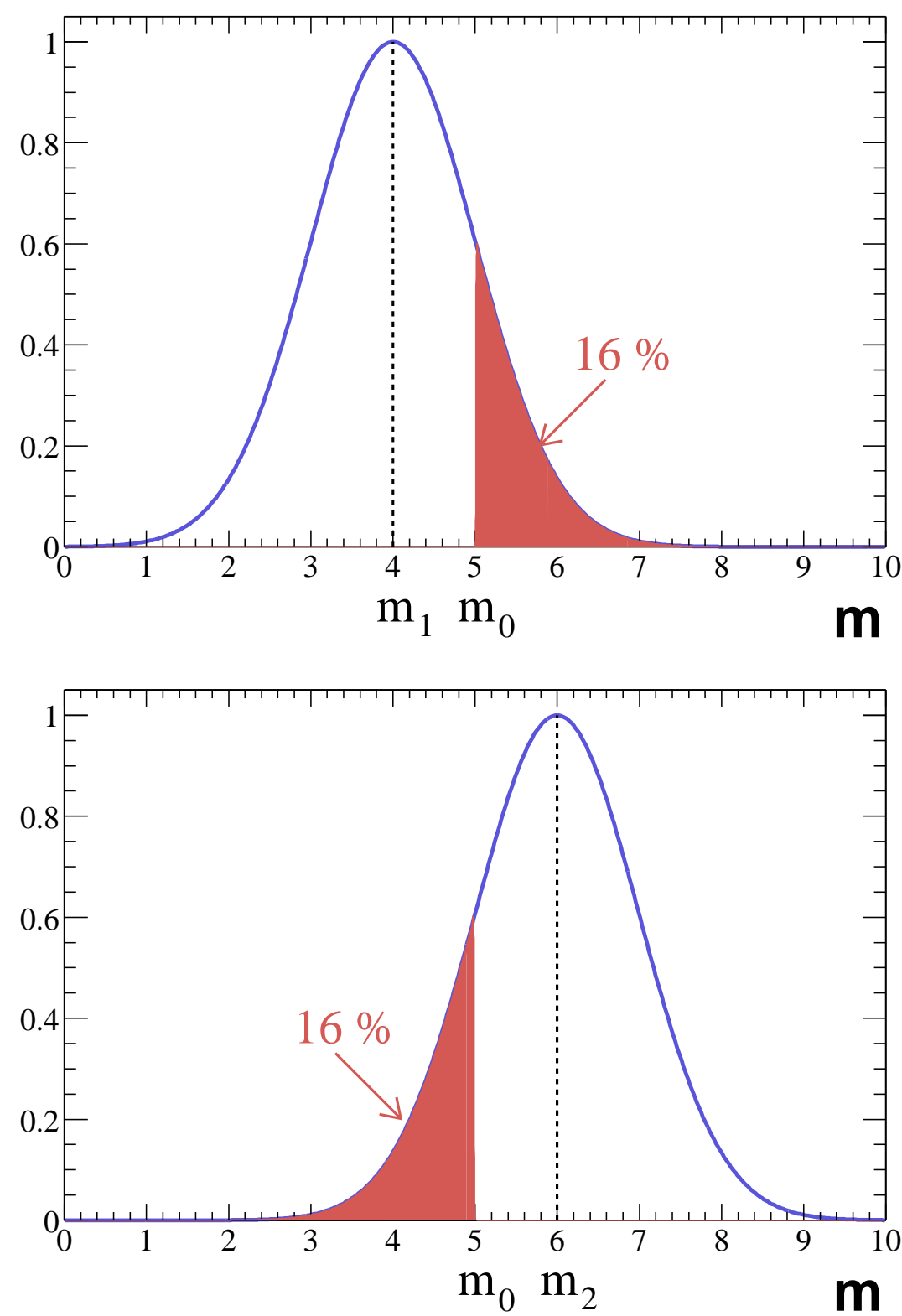

Fig. 6.2: Détermination de $m_{1}$ et $m_{2}$ pour $m_{0}=5$ et $\sigma_{m}=1$.

Il est important de remarquer que cette approche est totalement différente dans l'esprit de celle qui consiste à affirmer directement que si l'on mesure $m_{0} \pm \sigma_{m}$, alors l'intervalle de confiance à $68 \%$ est $\left(m_{1}=m_{0}-\sigma_{m}, m_{2}=m_{0}+\sigma_{m}\right)$. Dans le cas simple que l'on vient d'exposer, le résultat est le même, mais comme on le verra dans la suite, il existe des situations dans lesquelles ces deux approches conduisent à des résultats différents. 
Dans l'approche classique, on peut définir un $\chi^{2}$ centré en $m_{0}$ :

$$
\chi^{2}\left(m_{t}\right)=\frac{\left(m_{0}-m_{t}\right)^{2}}{2 \sigma_{m}^{2}}
$$

mais on ne doit pas interpréter ce $\chi^{2}$ comme une densité de probabilité. Pour construire un intervalle à $68 \%$ de niveau de confiance, on détermine $m_{1}$ et $m_{2}$ tels que $\Delta \chi^{2}\left(m_{1}\right)=$ $\Delta \chi^{2}\left(m_{2}\right)=1$, où $\Delta \chi^{2}(m)=\chi^{2}(m)-\chi_{\min }^{2}$. Dans le cas simple étudié ici, la valeur mesurée correspond à la valeur qui minimise le $\chi^{2}$ et donc $\chi_{\min }^{2}=\chi^{2}\left(m_{0}\right)$.

On considère la distribution de $\Delta \chi^{2}$ pour toutes les valeurs possibles que l'on peut mesurer. Pour cela, on génère des valeurs de $m$ en utilisant une distribution gaussienne centrée en $m_{0}$, de largeur $\sigma_{m}$. Pour chaque valeur $m_{i}$ ainsi générée, $\Delta \chi^{2}\left(m_{i}\right)=\chi^{2}\left(m_{i}\right)-$ $\chi^{2}\left(m_{0}\right)$. Dans le cas gaussien, $68 \%$ des valeurs générées ont un $\Delta \chi^{2}$ inférieur à 1 et l'ensemble de ces valeurs correspond à l'intervalle $\left(m_{1}, m_{2}\right)$. La distribution de $\Delta \chi^{2}$ est représentée figure 6.3 .

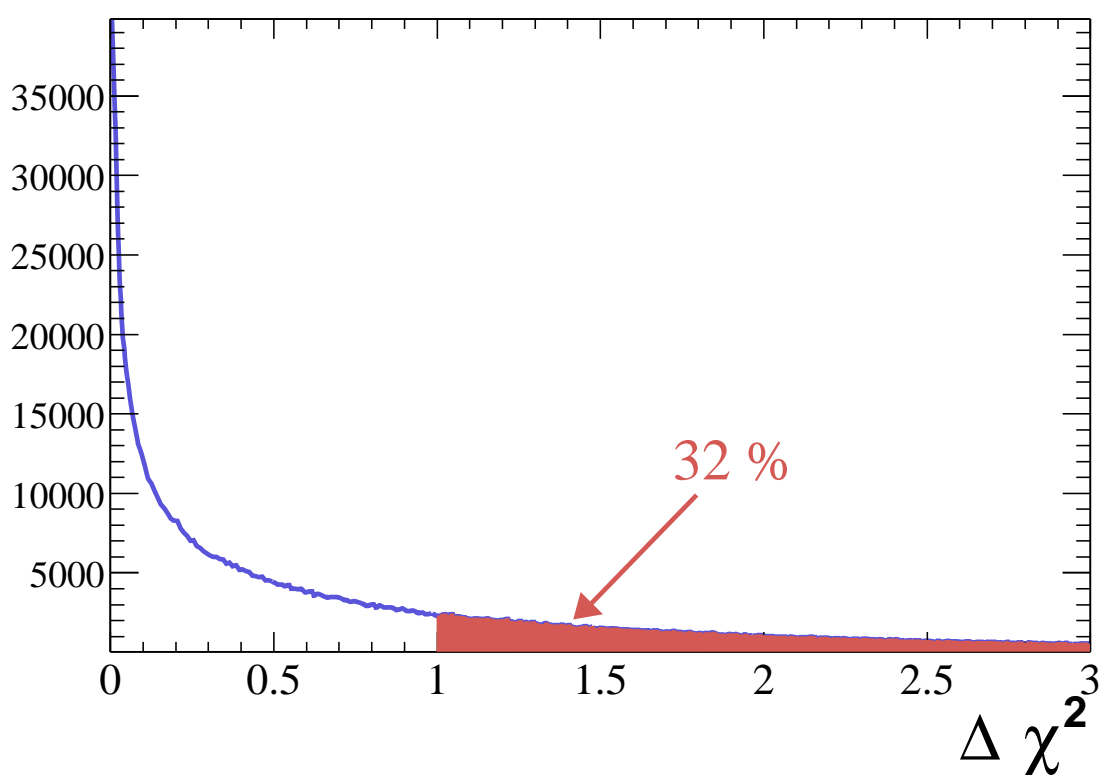

Fig. 6.3: Distribution de $\Delta \chi^{2}$ dans le cas gaussien. La partie colorée représente $32 \%$ de la distribution.

Cette figure a été réalisée avec $m_{0}=1$ et $\sigma_{m}=1$, ce qui conduit à $\left(m_{1}, m_{2}\right)=(0,2)$. On peut remarquer que dans le cas gaussien non borné, le choix de ces valeurs n'influe pas sur la distribution de $\Delta \chi^{2}$. On peut utiliser cette distribution de $\Delta \chi^{2}$ pour déterminer un intervalle de confiance pour toute valeur de $\alpha$. Pour cela, il suffit de déterminer la valeur de $\Delta \chi_{\alpha}^{2}$ telle qu'une fraction $\alpha$ des valeurs générées ait un $\Delta \chi^{2}$ inférieur à $\Delta \chi_{\alpha}^{2}$. L'ensemble des valeurs correspondantes représente l'intervalle avec un niveau de confiance $\alpha$. 


\subsubsection{Cas gaussien borné}

Dans l'exemple exposé au paragraphe précédent, on a déterminé $m_{1}$ et $m_{2}$ de manière totalement symétrique par rapport à $m_{0}$. On n'a, à aucun moment, restreint l'intervalle de valeurs que peut prendre le paramètre $m$. Il existe cependant des situations où le paramètre $m$ ne peut pas pas prendre n'importe quelle valeur, et ce, de façon totalement objective. Par exemple, si le paramètre $m$ correspond à une masse, il est clair que $m$ ne peut pas être négative. Lorsque l'on mesure l'observable physique, il est légitime de permettre à l'ajustement de choisir une valeur négative pour $m$, afin de tenir compte de la statistique et d'éventuelles erreurs systématiques. En revanche, lorsque l'on veut interpréter notre mesure de $m$ en termes d'intervalles de confiance, ces derniers, ainsi que leurs sous-ensembles, ne doivent pas être non-physiques. On génère donc des valeurs de $m$ de la même manière que dans le cas non borné $\left(m_{0}=1\right.$ et $\left.\sigma_{m}=1\right)$, sans restreindre l'intervalle que peuvent prendre les valeurs mesurées. En revanche, lorsque l'on calcule le $\chi^{2}$ pour chaque valeur $m_{i}$ générée, on empêche $m$ de prendre des valeurs négatives. Concrètement, cela signifie que si $m_{i}$ est hors de la région physique, alors la valeur du $\chi^{2}$ correspond à la valeur à la limite. Par conséquent, la distribution de $\Delta \chi^{2}$ est différente dans le cas gaussien borné par rapport au cas gaussien non borné. La figure 6.4 représente la distribution de $\Delta \chi^{2}$ pour les mêmes valeurs que précédemment, mais avec la borne $m>0$.

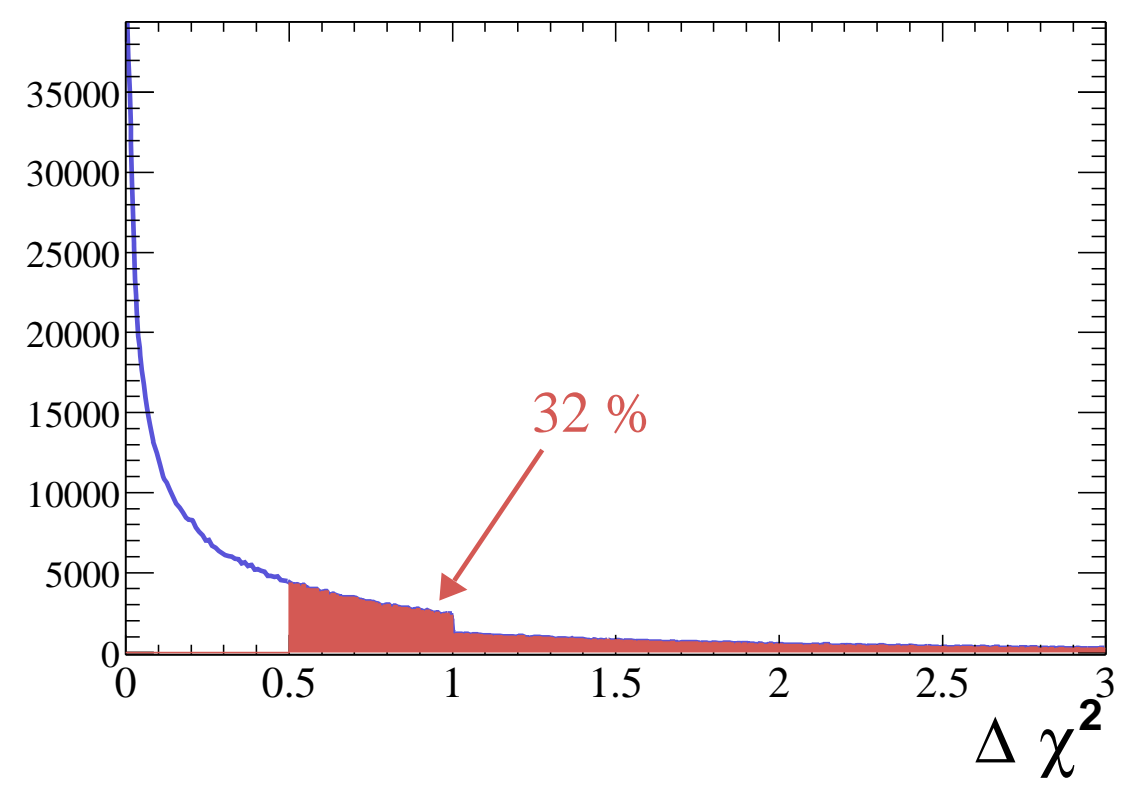

Fig. 6.4: Distribution de $\Delta \chi^{2}$ dans le cas gaussien borné. La partie colorée représente $32 \%$ de la distribution.

Dans ce cas-là, si on détermine un intervalle $\left(m_{1}, m_{2}\right)$ à $68 \%$ de niveau de confiance en cherchant $m_{1}$ et $m_{2}$ tels que $\Delta \chi^{2}\left(m_{1}\right)=\Delta \chi^{2}\left(m_{2}\right)=1$, on obtient un intervalle de confiance trop large, car $84 \%$ des valeurs générées ont un $\Delta \chi^{2}$ inférieur à 1 . La valeur de $\Delta \chi^{2}$ telle que $68 \%$ des valeurs générées aient un $\Delta \chi^{2}$ inférieur est en réalité 0,5.

Cet exemple simple a permis de montrer que dans certains cas, utiliser la variation 
d'une unité de la quantité $\Delta \chi^{2}$ pour déterminer un intervalle à $68 \%$ de niveau de confiance pouvait conduire à des intervalles de largeur surestimée. C'est en particulier vrai lorsque que l'on mesure un paramètre proche ou hors de la limite de la région physique. C'est ce constat qui a poussé Cousins et Feldman à développer une méthode d'interprétation fréquentiste des résultats [86], qui utilise la liberté inhérente à la construction de Neyman pour l'adapter au cas gaussien borné. Leur méthode permet de déterminer des intervalles de confiance qui ne sont jamais vides ou non physiques et qui ne sur-estiment ou ne sous-estiment pas un intervalle de confiance.

\subsection{Méthode de Feldman-Cousins}

La méthode de Feldman-Cousins est une méthode basée sur l'approche fréquentiste classique de Neyman qui permet de construire des intervalles de confiance. L'intérêt est que l'on s'affranchit alors de toute hypothèse sur le résultat. En particulier, on n'a pas besoin de déterminer a priori si on veut mettre une limite ou obtenir un intervalle de confiance. Cette méthode permet également de construire un échantillon complet d'intervalles de confiance, qui ne sont jamais vides ou non physiques. Deux situations extrêmes ont été étudiées par Feldman et Cousins : le cas des distributions poissonniennes, qui sera traité à titre informatif et le cas des distributions gaussiennes bornées, qui nous intéresse plus particulièrement dans le cadre de notre analyse.

\subsubsection{Distributions poissonniennes}

Cette section décrit la méthode proposée par Feldman-Cousins dans le cas où l'on cherche à extraire le nombre d'événements de signal parmi un petit nombre d'événements observés.

Dans ce cas-là, l'observable mesurée est le nombre total d'événements $n$. On suppose qu'on connaît le nombre d'événements de bruit de fond $b$ et on cherche à mettre un intervalle de confiance sur le nombre d'événements de signal $\mu$.

Pour mettre un intervalle de confiance sur un $\mu_{0}$ donné, on détermine pour chaque valeur de $n$ :

- la probabilité d'observer $n$ événements dont $\mu_{0}$ de signal :

$$
P\left(n \mid \mu_{0}\right)=\left(\mu_{0}+b\right)^{n} \exp \left(-\left(\mu_{0}+b\right)\right) / n !
$$

- le paramètre $\mu_{\text {best }}$ tel que $P\left(n \mid \mu_{\text {best }}\right.$ soit maximum : $\mu_{\text {best }}=\max (0, n-b)$

- le rapport des probabilités :

$$
R(n)=\frac{P\left(n \mid \mu_{0}\right)}{P\left(n \mid \mu_{\text {best }}\right)}
$$

On classe alors les $R(n)$ dans l'ordre décroissant et on additionne $P\left(n \mid \mu_{0}\right)$ dans cet ordre jusqu'à ce que la somme soit supérieure ou égale au niveau de confiance $\alpha$ que l'on veut mettre. Les valeurs de $\mathrm{n}$ correspondantes forment la région d'acceptance. $\mu_{0}$ appartient alors à cette région à $\alpha \%$ de niveau de confiance. On recommence la procédure pour chaque valeur de $\mu$, ce qui permet de construire un intervalle de confiance pour chaque $\mu$. 
Il peut être également intéressant de répéter le processus complet pour des nombres de bruits de fond $b$ différents, afin de s'affranchir des suppositions que l'on fait sur $b$.

\subsubsection{Distributions gaussiennes bornées}

Supposons que l'on dispose des mesures expérimentales des observables $x_{i}$ et que l'on veuille extraire un paramètre $\mu$. Le but est de déterminer un intervalle de confiance pour chaque valeur de $\mu$. Pour une valeur de $\mu_{0}$ donnée, on note $P\left(x \mid \mu_{0}\right)$ la probabilité de mesurer $x$. Le paramètre $\mu_{\text {best }}$ est défini comme étant le paramètre qui maximise cette probabilité. On contraint $\mu_{\text {best }}$ à être dans la région physique. On considère le rapport des maxima de vraisemblance :

$$
R(x)=\frac{P\left(x \mid \mu_{0}\right)}{P\left(x \mid \mu_{\text {best }}\right)} .
$$

De façon équivalente, on peut considérer la quantité :

$$
R^{\prime}(x)=\Delta \chi^{2}=\chi^{2}\left(x, \mu_{0}\right)-\chi^{2}\left(x, \mu_{\text {best }}\right),
$$

où $\chi^{2}(x, \mu)=-2 \ln (P(x \mid \mu))$.

La détermination d'un intervalle de confiance pour chaque valeur de $\mu$ se fait de la façon suivante :

- on calcule $R^{\prime}$, noté $\Delta \chi_{\text {data }}^{2}$, avec les observables $x_{i}$ mesurées dans les données;

- on génère les $x_{i}$ pour un grand nombre de pseudo-expériences avec la même sensibilité que dans les données et on calcule pour chacune de ces pseudo-expérience le $R^{\prime}$ correspondant, noté $\Delta \chi_{\text {toy }}^{2}$;

- on interprète la fraction de pseudo-expériences telles que $\Delta \chi_{\text {toy }}^{2}>\Delta \chi_{\text {data }}^{2}$ comme un niveau de confiance.

Intuitivement, on peut interpréter cette fraction comme étant la probabilité de mesurer des paramètres avec le même $\Delta \chi^{2}$ que celui des données, pour une valeur $\mu$ donnée.

La méthode utilisée pour interpréter notre mesure est fortement inspirée de cette méthode et sera détaillée dans la partie 6.3. Une différence fondamentale concerne la définition de la quantité $\Delta \chi^{2}$ que nous avons utilisée.

\subsubsection{Définition du $\Delta \chi^{2}$}

Nos mesures expérimentales dépendent de plusieurs paramètres : $r^{*}, \sin (2 \beta+\gamma)$ et $\delta^{*}$ et on cherche à déterminer des intervalles de confiance qui dépendent uniquement de $\sin (2 \beta+\gamma)$. On définit donc un $\Delta \chi^{2}$ qui permet de s'affranchir des "nuisances" $r^{*}$ et $\delta^{*}$. On minimise dans un premier temps le $\chi^{2}$ en ajustant les trois paramètres $r^{*}, 2 \beta+\gamma$ et $\delta^{*}$, puis on fixe $2 \beta+\gamma$ à une valeur que l'on veut tester et on minimise à nouveau le $\chi^{2}$ en ajustant les deux autres paramètres $r^{*}$ et $\delta^{*}$. Le $\Delta \chi^{2}$ que l'on utilise est alors la différence entre le $\chi^{2}$ au minimum de l'ajustement à deux paramètres et le $\chi^{2}$ au minimum de l'ajustement à trois paramètres. 


\subsection{Application au cas de la mesure de $\sin (2 \beta+\gamma)$}

On cherche à déterminer un intervalle de confiance sur $\sin (2 \beta+\gamma)$, à partir de nos mesures expérimentales. L'étude de la violation de $C P$ dans les désintégrations $B^{0} \rightarrow D^{*-} \pi^{+}$ partiellement reconstruites a conduit à la mesure de $a_{D^{*} \pi}^{L}$ et $c_{D^{*} \pi}^{L}$ pour les événements étiquetés par un lepton et de $a_{D^{*} \pi}^{K}, b_{D^{*} \pi}^{K}$ et $c_{D^{*} \pi}^{K}$ pour les événements étiquetés par un kaon avec :

$$
\begin{aligned}
a_{D^{*} \pi}^{L} & =2 r^{*} \sin (2 \beta+\gamma) \cos \delta^{*}=-0,042 \pm 0,019 \pm 0,010 \\
c_{D^{*} \pi}^{L} & =2 r^{*} \cos (2 \beta+\gamma) \sin \delta^{*}=-0,019 \pm 0,022 \pm 0,019 \\
a_{D^{*} \pi}^{K} & =2 r^{*} \sin (2 \beta+\gamma) \cos \delta^{*}=-0,025 \pm 0,020 \pm 0,015 \\
b_{D^{*} \pi}^{K} & =2 r^{\prime} \sin (2 \beta+\gamma) \cos \delta^{\prime}=-0,004 \pm 0,010 \pm 0,011 \\
c_{D^{*} \pi}^{K} & =2 \cos (2 \beta+\gamma)\left(r^{*} \sin \delta^{*}-r^{\prime} \sin \delta^{\prime}\right)=-0,002 \pm 0,020 \pm 0,016 .
\end{aligned}
$$

On en déduit la valeur combinée pour $a_{D^{*} \pi}: a_{D^{*} \pi}=-0,034 \pm 0,014 \pm 0,009$.

D'autre part, nous disposons de la mesure expérimentale de $r^{*}$ (que l'on note $r_{0}^{*}$ ), obtenue à partir de la mesure du rapport d'embranchement $\mathcal{B}\left(B^{0} \rightarrow D_{s}^{*} \pi\right)$ et en supposant la symétrie de saveur $S U(3)$ :

$$
r^{*}=0,015_{-0,006}^{+0,004}
$$

Cette mesure associée à la mesure de $a_{D^{*} \pi}$ tend à favoriser des grandes valeurs de $\sin (2 \beta+\gamma)$. On a donc une mesure proche de la limite physique, car $\sin (2 \beta+\gamma)$ est borné entre -1 et 1 , ce qui nous a amené à développer une méthode d'interprétation fréquentiste des résultats, afin d'interpréter la mesure de $\sin (2 \beta+\gamma)$ dans les désintégrations $B^{0} \rightarrow D^{*-} \pi^{+}$partiellement reconstruites. Pour cela on utilise les asymétries de $C P$ mesurées, données par l'équation 6.7. Puisque l'on ne dispose pas d'information sur les valeurs des paramètres effectifs $r^{\prime}$ et $\delta^{\prime}$, on n'utilise pas les mesures qui dépendent de ces paramètres, à savoir $b_{D^{*} \pi}^{K}$ et $c_{D^{*} \pi}^{K}$.

Dans une première méthode (partie 6.3.1), nous supposons que la symétrie $S U(3)$ est vraie avec une erreur théorique plate de $30 \%$ et nous utilisons donc la valeur calculée de $r^{*}$ donnée par l'équation 6.8. Dans un deuxième temps, nous nous affranchissons de toute hypothèse sur $r^{*}$ et nous reproduisons la même méthode en scannant différentes valeurs de $r^{*}($ partie 6.3.2).

\subsubsection{Méthode avec une hypothèse sur $S U(3)$}

Dans cette méthode, on utilise les mesures de $a_{D^{*} \pi}^{L}, c_{D^{*} \pi}^{L}, a_{D^{*} \pi}^{K}$ et de $r^{*}$. Les paramètres $a_{D^{*} \pi}^{L}, c_{D^{*} \pi}^{L}, a_{D^{*} \pi}^{K}$ étant corrélés, on utilise une matrice des erreurs $V$ calculée de la façon suivante : $V=V_{\text {stat }}+V_{\text {systC }}+V_{\text {syst } N C}$, où

- $V_{\text {stat }}$ est la matrice des erreurs statistiques provenant de l'ajustement sur les données. Puisque l'ajustement sur les événements étiquetés par un lepton et l'ajustement sur 
les événements étiquetés par un kaon sont indépendants, il n'y a pas de corrélation entre $a_{D^{*} \pi}^{K}$ et les paramètres $a_{D^{*} \pi}^{L}$ et $c_{D^{*} \pi}^{L}$. En revanche, ces deux derniers sont corrélés, mais très faiblement $(0,6 \%)$. La matrice $V_{\text {stat }}$ est donc quasiment diagonale. Son expression générale est :

$$
\left.\begin{array}{ccc}
V_{\text {stat }}= & \\
\sigma_{\text {stat }}^{2}\left(a_{D^{*} \pi}^{L}\right) & 0,006 \sigma_{\text {stat }}\left(a_{D^{*} \pi}^{L}\right) \sigma_{\text {stat }}\left(c_{D^{*} \pi}^{L}\right) & 0 \\
0,006 \sigma_{\text {stat }}\left(a_{D^{*} \pi}^{L}\right) \sigma_{\text {stat }}\left(c_{D^{*} \pi}^{L}\right) & \sigma_{\text {stat }}^{2}\left(c_{D^{*} \pi}^{L}\right) & 0 \\
0 & 0 & \sigma_{\text {stat }}^{2}\left(a_{D^{*} \pi}^{K}\right)
\end{array}\right)
$$

avec

$$
\begin{aligned}
\sigma_{\text {stat }}\left(a_{D^{*} \pi}^{L}\right) & =0,019, \\
\sigma_{\text {stat }}\left(c_{D^{*} \pi}^{L}\right) & =0,022, \\
\sigma_{\text {stat }}\left(a_{D^{*} \pi}^{K}\right) & =0,020 .
\end{aligned}
$$

- $V_{\text {systC }}$ est la matrice des erreurs systématiques corrélées, alors que $V_{\text {syst } N C}$ est la matrice des erreurs systématiques non corrélées. On considère que les erreurs systématiques liées à la violation de $C P$ dans le bruit de fond et au détecteur sont corrélées à $100 \%$, alors que les autres types d'erreurs systématiques ne sont pas corrélés. On note $\sigma_{\text {systC }}$ l'erreur systématique corrélée et $\sigma_{\text {syst } N C}$ l'erreur systématique non corrélée. Les expressions de $V_{\text {systC }}$ et $V_{\text {systNC }}$ sont :

$V_{\text {syst } C}=$

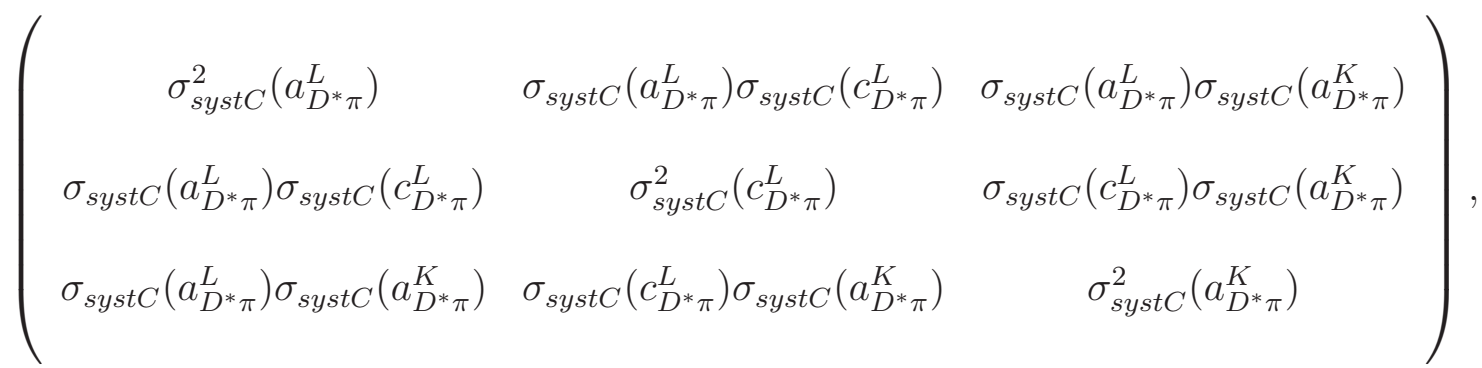

$$
\begin{aligned}
& V_{\text {syst NC }}= \\
& \left(\begin{array}{ccc}
\sigma_{\text {syst } N C}^{2}\left(a_{D^{*} \pi}^{L}\right) & 0 & 0 \\
0 & \sigma_{\text {syst } N C}^{2}\left(c_{D^{*} \pi}^{L}\right) & 0 \\
0 & 0 & \sigma_{\text {syst NC }}^{2}\left(a_{D^{*} \pi}^{K}\right)
\end{array}\right) ;
\end{aligned}
$$


avec

$$
\begin{aligned}
& \sigma_{\text {syst } C}\left(a_{D^{*} \pi}^{L}\right)=0,0074, \\
& \sigma_{\text {syst } C}\left(c_{D^{*} \pi}^{L}\right)=0,0088, \\
& \sigma_{\text {syst } C}\left(a_{D^{*} \pi}^{K}\right)=0,0105 . \\
& \sigma_{\text {syst } N C}\left(a_{D^{*} \pi}^{L}\right)=0,0066, \\
& \sigma_{\text {syst } N C}\left(c_{D^{*} \pi}^{L}\right)=0,0084, \\
& \sigma_{\text {syst } N C}\left(a_{D^{* \pi}}^{K}\right)=0,0081 .
\end{aligned}
$$

On construit un $\chi^{2}$ qui utilise ces mesures et qui dépend de $2 \beta+\gamma, r^{*}$ et $\delta^{*}$ :

$$
\chi^{2}=\sum_{j, k=1}^{3} \Delta x_{j} V_{j k}^{-1} \Delta x_{k}+\chi^{2}\left(r^{*}\right),
$$

où $\Delta x_{j}$ est la différence entre le paramètre mesuré et l'expression théorique de ce paramètre, pour $a_{D^{*} \pi}^{L}(j=1), c_{D^{*} \pi}^{L}(j=2)$ et $a_{D^{*} \pi}^{K}(j=3)$ :

$$
\begin{aligned}
& \Delta x_{1}=a_{D^{*} \pi}^{L}-2 r^{*} \sin (2 \beta+\gamma) \cos \delta^{*}, \\
& \Delta x_{2}=c_{D^{*} \pi}^{L}-2 r^{*} \cos (2 \beta+\gamma) \sin \delta^{*}, \\
& \Delta x_{3}=a_{D^{*} \pi}^{K}-2 r^{*} \sin (2 \beta+\gamma) \cos \delta^{*}
\end{aligned}
$$

et $\chi^{2}\left(r^{*}\right)$ est le terme spécifique à la mesure expérimentale de $r^{*}$. On tient compte de l'erreur théorique plate de $30 \%$ due à l'hypothèse sur $S U(3)$, ainsi que des erreurs expérimentales, que l'on assume être gaussiennes. Ce terme n'est pas corrélé aux autres mesures. On a ainsi l'expression suivante :

$$
\begin{aligned}
\chi^{2}\left(r^{*}\right) & =\frac{\left(r^{*}-0,7 r_{0}^{*}\right)^{2}}{\sigma^{2}\left(r_{0}^{*}\right)}, & r^{*}<0,7 r_{0}^{*} \\
\chi^{2}\left(r^{*}\right) & =\frac{\left(r^{*}-1,3 r_{0}^{*}\right)^{2}}{\sigma^{2}\left(r_{0}^{*}\right)}, & r^{*}>1,3 r_{0}^{*} \\
\chi^{2}\left(r^{*}\right) & =0, & 0,7 r_{0}^{*}<r^{*}<1,3 r_{0}^{*}
\end{aligned}
$$

On minimize alors le $\chi^{2}$ en ajustant les paramètres $2 \beta+\gamma, r^{*}$ et $\delta^{*}$. On note $\chi_{\min }^{2}$ la valeur $\mathrm{du} \chi^{2}$ au minimum.

On cherche à attribuer un intervalle de confiance sur $\sin (2 \beta+\gamma)$ avec un niveau de confiance que l'on doit déterminer pour chaque valeur de $2 \beta+\gamma$. En réalité, on détermine un intervalle de confiance qui dépend de $|\sin (2 \beta+\gamma)|$, car il y a une ambigüité entre le signe de $2 \beta+\gamma$ et le signe de $\delta^{*}$. On réalise un scan des valeurs possibles de $2 \beta+\gamma$. En pratique, on peut se limiter à l'intervalle $[0, \pi / 2]$, car il existe des ambigüités supplémentaire entre les deux paramètres qui correspondent à des angles : $2 \beta+\gamma$ et $\delta^{*}$. En effet, les couples 
$\left(2 \beta+\gamma, \delta^{*}\right),\left(\pi-2 \beta+\gamma,-\delta^{*}\right)$ et $\left(2 \beta+\gamma+\pi, \delta^{*}+\pi\right)$ conduisent à la même valeur de $|\sin (2 \beta+\gamma)|$.

On a ainsi un échantillon de valeurs de $2 \beta+\gamma$ et on détermine un niveau de confiance pour chaque intervalle, de la façon exposée ci-après. Comme notre mesure est proche de la limite de la région physique, avec une grande erreur, l'intervalle que l'on considère pour une valeur de $2 \beta+\gamma$ donnée est l'intervalle $(|\sin (2 \beta+\gamma)|, 1)$. En clair, on détermine d'abord l'intervalle et on cherche ensuite à déterminer son niveau de confiance.

Soit $x_{i}$ une valeur fixée de $2 \beta+\gamma$ :

1. On fixe $2 \beta+\gamma=x_{i}$ et on minimize à nouveau le $\chi^{2}$, mais en ajustant uniquement les paramètres $r^{*}$ et $\delta^{*}$.

2. On note $\chi_{\min }^{\prime 2}\left(x_{i}\right)$ la valeur de ce $\chi^{2}$ au minimum et $r_{\min }^{*}\left(x_{i}\right)$ et $\delta_{\min }^{*}\left(x_{i}\right)$ les valeurs ajustées de $r^{*}$ et $\delta^{*}$.

3. On définit alors la quantité $\Delta \chi^{2}\left(x_{i}\right)=\chi_{m i n}^{\prime 2}\left(x_{i}\right)-\chi_{m i n}^{2}$.

On génère alors un grand nombre de pseudo-expériences en utilisant les erreurs expérimentales et en tenant compte des corrélations entre $a_{D^{*} \pi}^{L}, c_{D^{*} \pi}^{L}$ et $a_{D^{*} \pi}^{K}$. On note $\vec{p}$ le vecteur de paramètres à trois composantes que l'on veut générer. On génère en réalité une déviation de ces paramètres par rapport à leur valeur centrale. On note $\vec{m}$ le vecteur des valeurs centrales et $\vec{\epsilon}$ le vecteur des déviations. On a donc : $\vec{p}=\vec{m}+\vec{\epsilon}$, avec :

$$
\vec{m}=\left(\begin{array}{l}
a_{\text {moy }} \\
c_{\text {moy }} \\
a_{\text {moy }}
\end{array}\right)=\left(\begin{array}{l}
2 r_{\text {min }}^{*}\left(x_{i}\right) \sin \left(x_{i}\right) \cos \left(\delta_{\text {min }}^{*}\left(x_{i}\right)\right) \\
2 r_{\text {min }}^{*}\left(x_{i}\right) \cos \left(x_{i}\right) \sin \left(\delta_{\text {min }}^{*}\left(x_{i}\right)\right) \\
2 r_{\text {min }}^{*}\left(x_{i}\right) \sin \left(x_{i}\right) \cos \left(\delta_{\text {min }}^{*}\left(x_{i}\right)\right)
\end{array}\right) .
$$

On génère le vecteur des déviations de la façon suivante :

1. On diagonalise la matrice des erreurs $V$, afin de travailler dans une base où les paramètres ne sont pas corrélés. On appelle $D$ la matrice des erreurs dans cette base et $T$ la matrice de changement de base. On a ainsi $D \equiv T V T^{-1}$.

2. On génère les variations $\epsilon_{i}$ de chaque paramètre $i(i=1,2,3)$ en utilisant une gaussienne centrée en 0 , de largeur $\sqrt{D_{i i}}$. On appelle $\vec{\epsilon} \vec{\epsilon}^{\prime}$ le vecteur de ces variations.

3. On en déduit le vecteur de ces variations dans la base de départ: $\vec{\epsilon}=T^{-1} \vec{\epsilon}^{\prime}$.

Enfin, $r^{*}$ est généré à l'aide d'une distribution centrée en $r_{\min }^{*}\left(x_{i}\right)$, qui est plate entre $0,7 r_{\min }^{*}\left(x_{i}\right)$ et $1,3 r_{\min }^{*}\left(x_{i}\right)$ et qui est gaussienne ailleurs.

Pour chaque lot $j$ de mesures ainsi généré on applique la même procédure que pour les données et on calcule la quantité $\Delta \chi^{2}\left(x_{i}\right)_{j}$, où $j$ représente le numéro de la pseudoexpérience considérée.

Ainsi, pour chaque $x_{i}$, on dispose d'une valeur de $\Delta \chi^{2}\left(x_{i}\right)$ calculée en utilisant les mesures expérimentales et d'un grand nombre de valeurs de $\Delta \chi^{2}\left(x_{i}\right)_{j}$, calculées en simulant des pseudo-expériences. La fraction de pseudo-expériences telles que $\Delta \chi^{2}\left(x_{i}\right)_{j}>\Delta \chi^{2}\left(x_{i}\right)$ est interprétée comme un niveau de confiance correspondant à l'intervalle $\left(2 \beta+\gamma=x_{i}, 1\right)$. 
La procédure a été réalisée pour 100 valeurs de $2 \beta+\gamma$ comprises entre 0 et $\pi$. Le niveau de confiance en fonction de $|\sin (2 \beta+\gamma)|$ est représenté figure 6.5. On peut également mettre les limites suivantes:

- $|\sin (2 \beta+\gamma)|>0.35$ à $90 \%$ de niveau de confiance,

- $|\sin (2 \beta+\gamma)|>0.62$ à $68 \%$ de niveau de confiance.

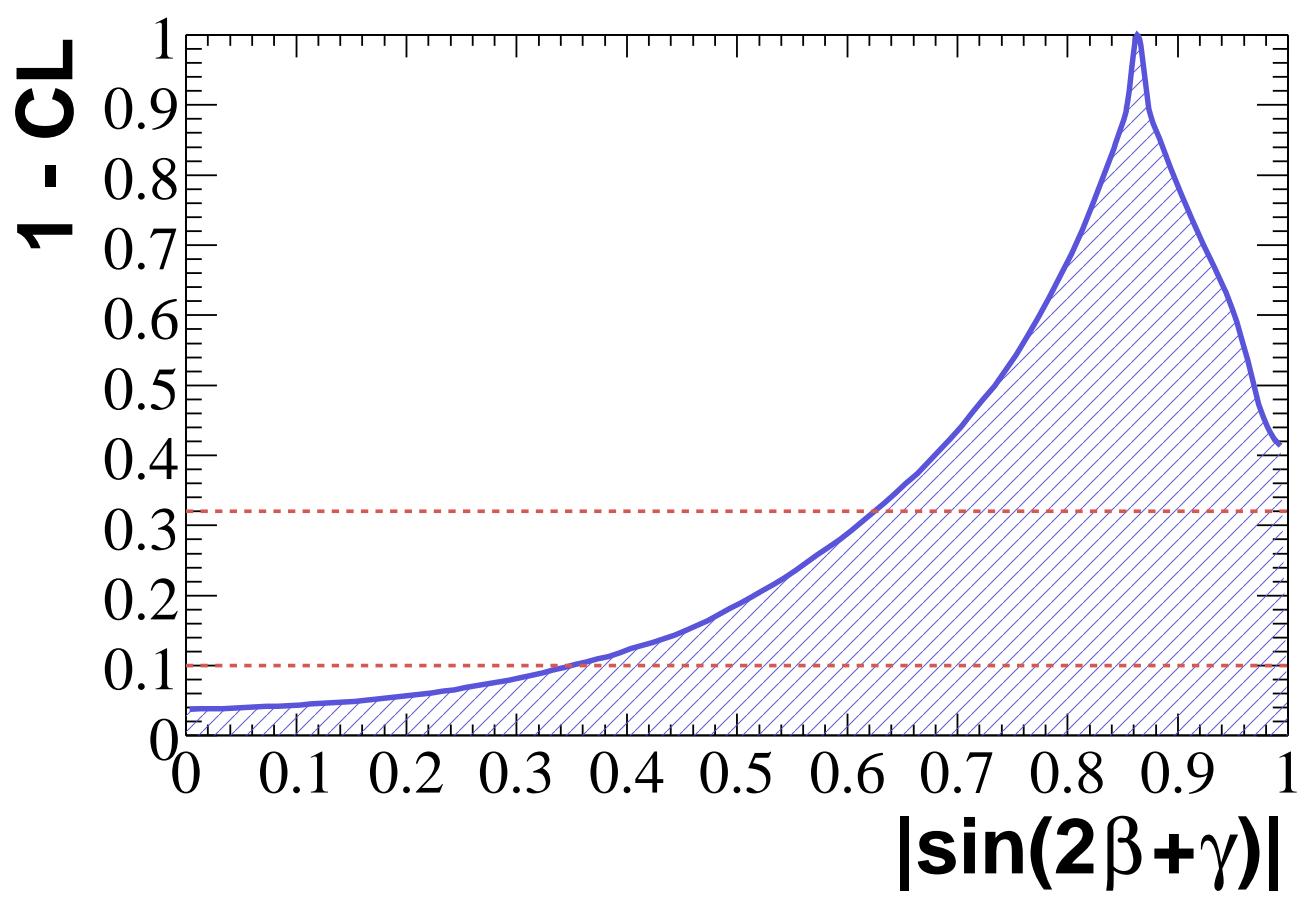

FIG. 6.5: Niveau de confiance en fonction de $|\sin (2 \beta+\gamma)|$. Ce graphe doit se lire de la façon suivante : pour une valeur donnée $x_{i}$ de $|\sin (2 \beta+\gamma)|$, on lit le niveau de confiance $\alpha_{i}$ correspondant et on peut alors affirmer que $|\sin (2 \beta+\gamma)|>x_{i}$ avec un niveau de confiance $\alpha_{i}$.

On en déduit également des contours de probabilité pour la position du sommet du triangle d'unitarité dans le plan $(\bar{\rho}, \bar{\eta})$, comme le montre la figure 6.6.

\section{Niveau de confiance en fonction de $\gamma$}

Il est maintenant possible de déterminer un niveau de confiance en fonction de $\gamma$, en combinant le niveau de confiance obtenu précédemment avec les mesures de $\sin (2 \beta)$ et de $\cos (2 \beta)$. On a utilisé ici la moyenne mondiale de $\sin (2 \beta)$ obtenue avec tous les modes charmonium : $\sin (2 \beta)=0,725 \pm 0,037[89,90]$ et la moyenne mondiale de $\cos (2 \beta)$ obtenue grâce au mode $B^{0} \rightarrow J / \Psi K^{*}: \cos (2 \beta)=1,69 \pm 0,67[91,92]$. Le niveau de confiance en fonction de $\gamma$ est représenté figure 6.7 . 


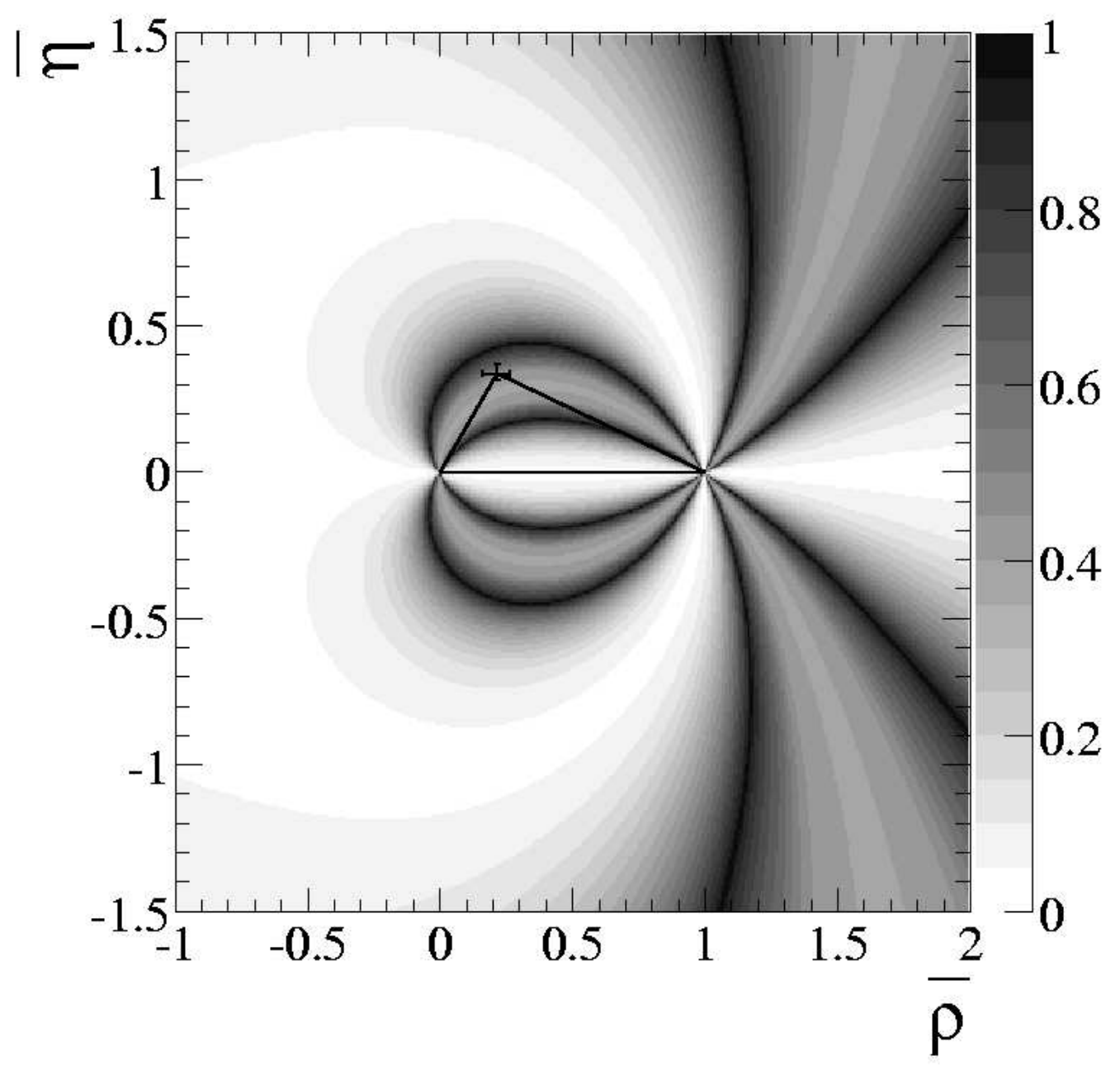

Fig. 6.6: Contours de probabilité constante que la position du sommet du triangle d'unitarité soit à l'intérieur du contour. La croix représente la valeur et les erreurs de la position du sommet du triangle, obtenue par CKMFitter [30]. 


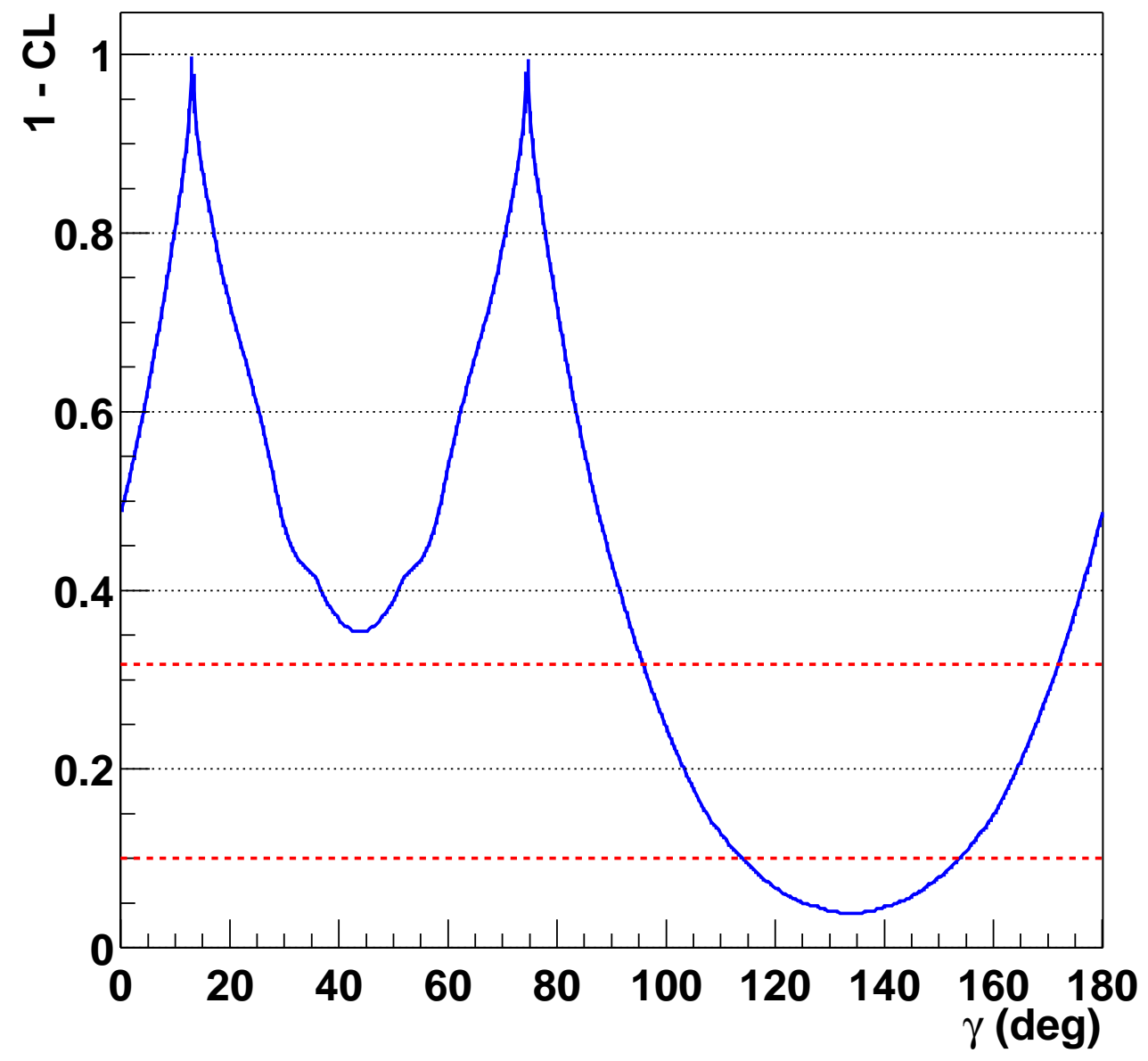

FIG. 6.7: Niveau de confiance en fonction de $\gamma$. 


\subsubsection{Méthode avec un scan de $r^{*}$}

Afin de nous affranchir de toute hypothèse sur la symétrie $S U(3)$, nous avons reproduit une méthode similaire en fixant $r^{*}$ à différentes valeurs. Pour cela, nous avons réalisé un scan de $r^{*}$ pour des valeurs comprises entre 0,005 et 0,05 . Nous pouvons ainsi obtenir des limites inférieures sur $|\sin (2 \beta+\gamma)|$ en fonction de $r^{*}$. Dans cette approche, nous supprimons le terme $\chi^{2}\left(r^{*}\right)$, dépendant de $r^{*}$ dans la définition du $\chi^{2}$. La valeur du paramètre $r^{*}$ n'est alors plus ajustée pour déterminer la quantité $\Delta \chi^{2}$, mais est fixée à la valeur scannée. On utilise également cette valeur pour générer les pseudo-expériences. La limite inférieure sur $|\sin (2 \beta+\gamma)|$ en fonction de $r^{*}$ avec un niveau de confiance de $90 \%$ est montrée figure 6.8 .

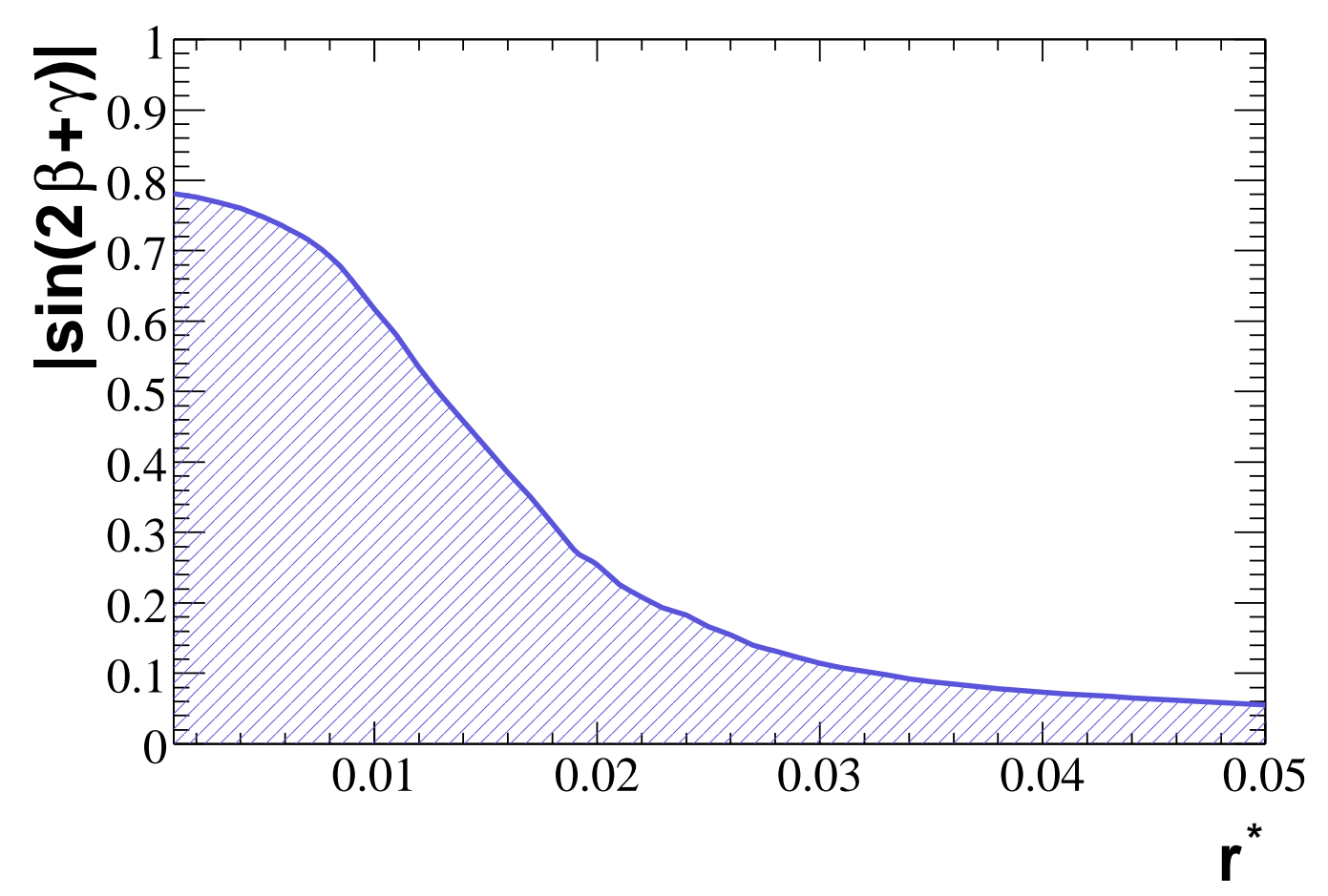

FIG. 6.8: Limite inférieure sur $|\sin (2 \beta+\gamma)|$ en fonction de $r^{*}$ avec un niveau de confiance de $90 \%$. 


\subsection{Combinaisons des différentes mesures de $\sin (2 \beta+$ $\gamma)$ par BABAR}

D'autres analyses de $B A B A R$ ont permis de mesurer $\sin (2 \beta+\gamma)$ dans les modes $B^{0} \rightarrow$ $D^{*} \pi, B^{0} \rightarrow D \pi$ et $B^{0} \rightarrow D \rho$ avec une méthode de reconstruction totale [93], qui utilise les données des Runs 1 à 3 de $B A B A R$, ce qui correspond à une luminosité intégrée de $113 \mathrm{fb}^{-1}$. Ces analyses fournissent pour chaque mode une mesure de $a$ pour toutes les catégories d'étiquetages confondues et une mesure de $c$ réalisée uniquement avec les événements étiquetés par un lepton. Ainsi, ces paramètres dépendent uniquement de $\sin (2 \beta+\gamma)$, de la phase forte relative $\left(\delta_{D^{*} \pi}, \delta_{D \pi}\right.$ et $\left.\delta_{D \rho}\right)$ et du rapport $r\left(r_{D^{*} \pi}, r_{D \pi}\right.$ et $\left.r_{D \rho}\right)$ de chaque mode. Les résultats sont résumés ici :

$$
\begin{aligned}
& a_{D^{*} \pi}=2 r_{D^{*} \pi} \sin (2 \beta+\gamma) \cos \left(\delta_{D^{*} \pi}\right)=-0,049 \pm 0,031 \pm 0,020 \\
& c_{D^{*} \pi}=2 r_{D^{*} \pi} \cos (2 \beta+\gamma) \sin \left(\delta_{D^{*} \pi}\right)=0,044 \pm 0,054 \pm 0,033 \\
& a_{D \pi}=2 r_{D \pi} \sin (2 \beta+\gamma) \cos \left(\delta_{D \pi}\right)=-0,032 \pm 0,031 \pm 0,020 \\
& c_{D \pi}=2 r_{D \pi} \cos (2 \beta+\gamma) \sin \left(\delta_{D \pi}\right)=-0,059 \pm 0,055 \pm 0,033 \\
& a_{D \rho}=2 r_{D \rho} \sin (2 \beta+\gamma) \cos \left(\delta_{D \rho}\right)=-0,005 \pm 0,044 \pm 0,021 \\
& c_{D \rho}=2 r_{D \rho} \cos (2 \beta+\gamma) \sin \left(\delta_{D \rho}\right)=-0,147 \pm 0,074 \pm 0,035
\end{aligned}
$$

On dispose également des valeurs du rapport $r$ pour chaque mode, obtenues de la même façon que celle exposée dans la partie 4.2 .3 :

$$
\begin{aligned}
& r_{D^{*} \pi}=0,015_{-0,004}^{+0,006} \\
& r_{D \pi}=0,020 \pm 0,003 \\
& r_{D \rho}=0,003 \pm 0,006
\end{aligned}
$$

On construit alors un $\chi^{2}$ analogue à celui utilisé pour l'interprétation des résultats présentés dans cette thèse, en ajoutant des termes supplémentaires pour chaque mesure. La partie du $\chi^{2}$ relative au rapport $r$ de chaque mode est analogue à l'équation 6.13 et tient compte de l'incertitude théorique de $30 \%$ due à l'hypothèse sur $S U(3)$.

Le niveau de confiance obtenu en combinant toutes les mesures réalisées avec une méthode de reconstruction totale de BABAR est représenté figure 6.9. La combinaison de ces mesures seules ne permet pas de mettre une limite sur $|\sin (2 \beta+\gamma)|$.

En ajoutant les résultats de l'analyse avec une reconstruction partielle, on obtient la figure 6.10. On en déduit que $\mid \sin (2 \beta+\gamma)>0,06(0,48)$ avec un niveau de confiance de $90 \%(68 \%)$. 


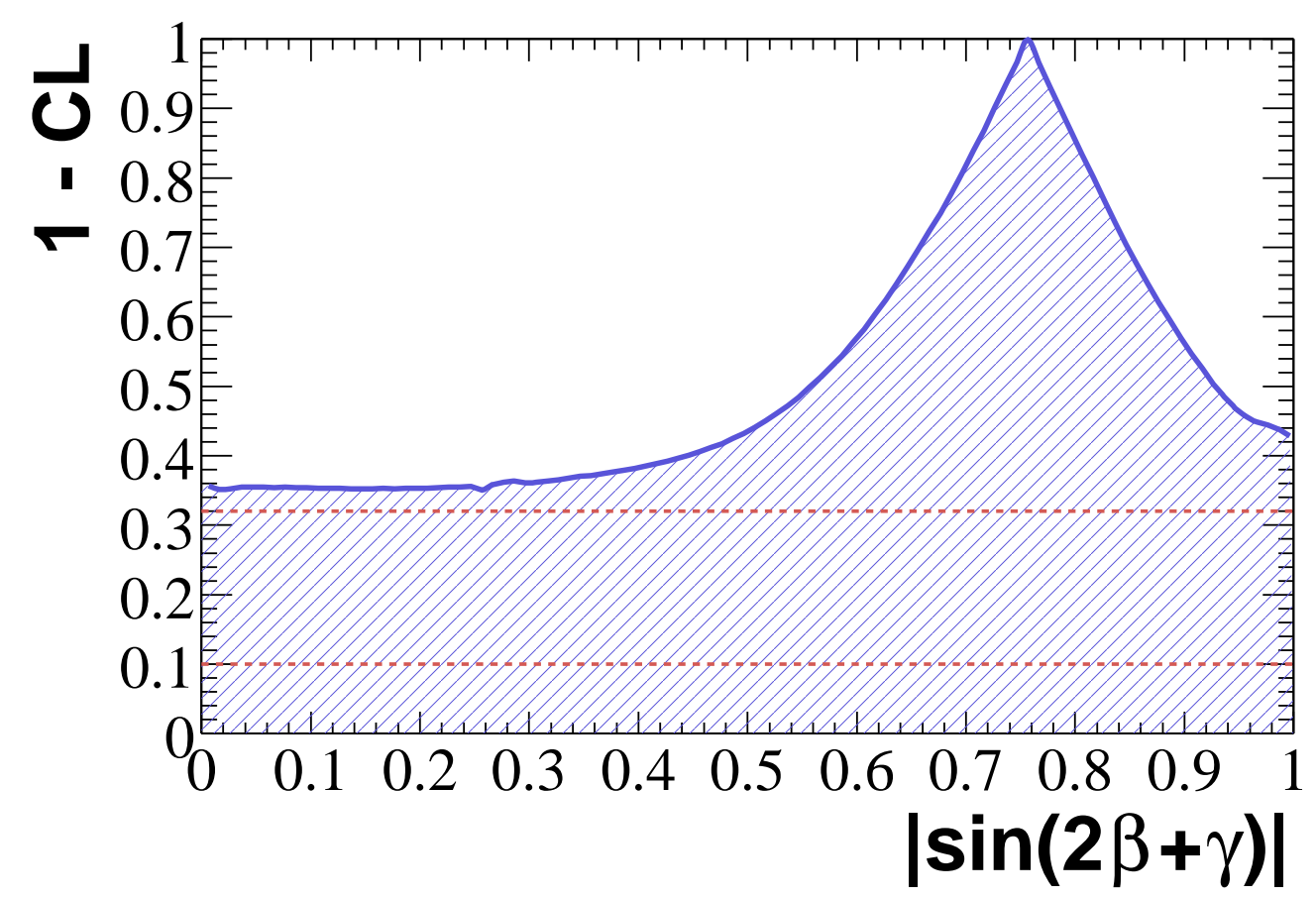

Fig. 6.9: Niveau de confiance en fonction de $|\sin (2 \beta+\gamma)|$ obtenu en combinant toutes les mesures dépendant de $\sin (2 \beta+\gamma)$, réalisées avec une méthode de reconstruction totale, de BABAR.

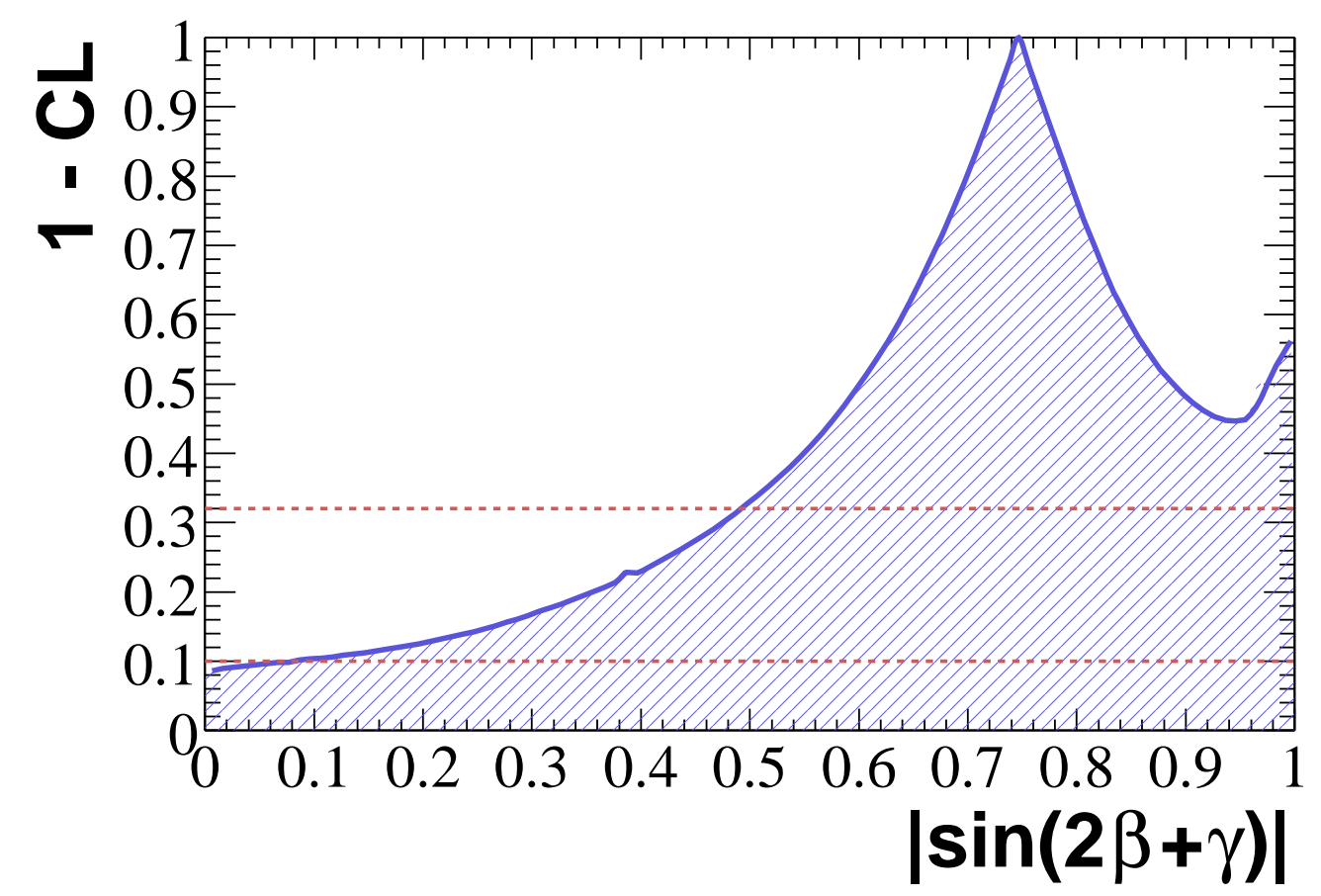

Fig. 6.10: Niveau de confiance en fonction de $|\sin (2 \beta+\gamma)|$ obtenu en combinant toutes les mesures dépendant de $\sin (2 \beta+\gamma)$ de $B A B A R$. 


\subsection{Combinaison mondiale de toutes les mesures de $\sin (2 \beta+\gamma)$}

L'expérience Belle a également mesuré $\sin (2 \beta+\gamma)$ dans le mode $B^{0} \rightarrow D^{*-} \pi^{+}$avec une méthode de reconstruction partielle et dans les modes $B^{0} \rightarrow D^{*-} \pi^{+}$et $B^{0} \rightarrow D^{-} \pi^{+}$ avec une méthode de reconstruction totale.

L'analyse de la violation de $C P$ dans les désintégrations $B^{0} \rightarrow D^{*-} \pi^{+}$partiellement reconstruites réalisée par Belle n'utilise que les événements étiquetés par un lepton provenant d'un échantillon de données dont la luminosité intégrée est de $140 \mathrm{fb}^{-1}$. Cela a conduit aux résultats suivants [94] :

$$
\begin{aligned}
& a_{D^{*} \pi}=2 r_{D^{*} \pi} \sin (2 \beta+\gamma) \cos \left(\delta_{D^{*} \pi}\right)=-0,031 \pm 0,028 \pm 0,018 \\
& c_{D^{*} \pi}=2 r_{D^{*} \pi} \cos (2 \beta+\gamma) \sin \left(\delta_{D^{*} \pi}\right)=-0,004 \pm 0,028 \pm 0,018
\end{aligned}
$$

L'analyse de Belle, qui utilise une méthode de reconstruction totale, porte sur un échantillon de données de luminosité intégrée de $140 \mathrm{fb}^{-1}$ et utilise tous les événements étiquetés disponibles. Les résultats sont les suivants [95] :

$$
\begin{aligned}
& a_{D^{*} \pi}=2 r_{D^{*} \pi} \sin (2 \beta+\gamma) \cos \left(\delta_{D^{*} \pi}\right)=0,060 \pm 0,040 \pm 0,019 \\
& c_{D^{*} \pi}=2 r_{D^{*} \pi} \cos (2 \beta+\gamma) \sin \left(\delta_{D^{*} \pi}\right)=0,049 \pm 0,040 \pm 0,019 \\
& a_{D \pi}=2 r_{D \pi} \sin (2 \beta+\gamma) \cos \left(\delta_{D \pi}\right)=-0,062 \pm 0,037 \pm 0,018 \\
& c_{D \pi}=2 r_{D \pi} \cos (2 \beta+\gamma) \sin \left(\delta_{D \pi}\right)=-0,025 \pm 0,037 \pm 0,018
\end{aligned}
$$

En combinant toutes les mesures de $B A B A R$ et de Belle relatives à $\sin (2 \beta+\gamma)$, on obtient les moyennes mondiales suivantes:

$$
\begin{aligned}
& a_{D^{*} \pi}=2 r_{D^{*} \pi} \sin (2 \beta+\gamma) \cos \left(\delta_{D^{*} \pi}\right)=-0,027 \pm 0,013 \\
& c_{D^{*} \pi}=2 r_{D^{*} \pi} \cos (2 \beta+\gamma) \sin \left(\delta_{D^{*} \pi}\right)=0,0015 \pm 0,018 \\
& a_{D \pi}=2 r_{D \pi} \sin (2 \beta+\gamma) \cos \left(\delta_{D \pi}\right)=-0,045 \pm 0,027 \\
& c_{D \pi}=2 r_{D \pi} \cos (2 \beta+\gamma) \sin \left(\delta_{D \pi}\right)=-0,035 \pm 0,035 \\
& a_{D \rho}=2 r_{D \rho} \sin (2 \beta+\gamma) \cos \left(\delta_{D \rho}\right)=-0,005 \pm 0,049 \\
& c_{D \rho}=2 r_{D \rho} \cos (2 \beta+\gamma) \sin \left(\delta_{D \rho}\right)=-0,147 \pm 0,082
\end{aligned}
$$

On peut alors interpréter ces mesures avec la même méthode fréquentiste, et on obtient le niveau de confiance représenté à la figure 6.11. On en déduit que $\mid \sin (2 \beta+\gamma)>0,28$ $(0,50)$ avec un niveau de confiance de $90 \%$ (68\%). 


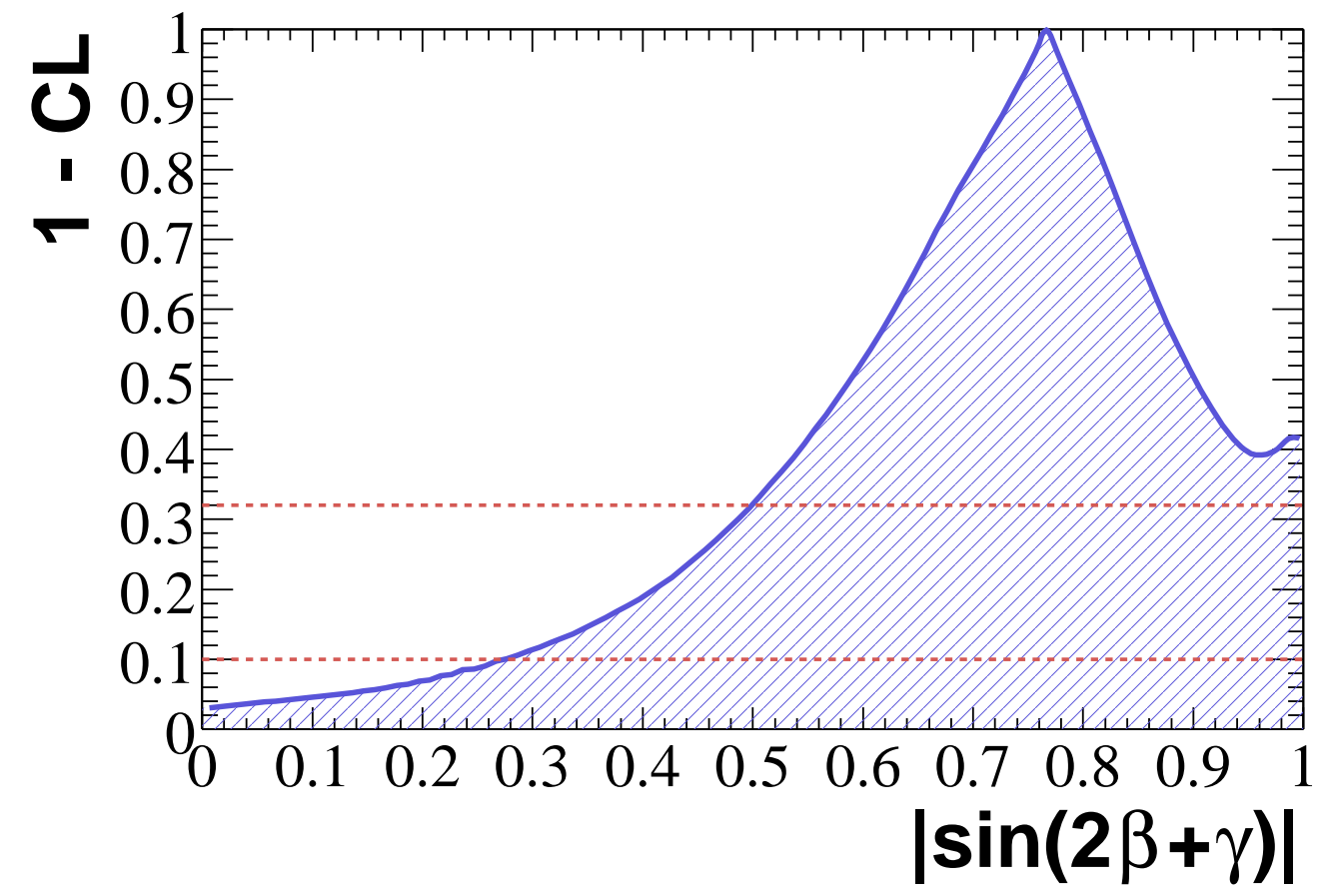

FIG. 6.11: Niveau de confiance en fonction de $|\sin (2 \beta+\gamma)|$ obtenu en combinant toutes les mesures dépendant de $\sin (2 \beta+\gamma)$ de $B A B A R$ et de Belle. 
Chapitre 6. Interprétation des résultats 


\section{Conclusion}

\section{Analyse de la violation de $C P$ dans $B \rightarrow D^{* \mp} \pi^{ \pm}$}

L'analyse de la violation de $C P$ dépendant du temps, dans les désintégrations $B \rightarrow$ $D^{* \mp} \pi^{ \pm}$partiellement reconstruites, utilisant un échantillon de données de $211 \mathrm{fb}^{-1}$ prises à la résonance $\Upsilon(4 S)$, a permis la mesure des paramètres :

$$
\begin{aligned}
a_{D^{*} \pi}^{L} & =2 r^{*} \sin (2 \beta+\gamma) \cos \delta=-0,042 \pm 0,019 \pm 0,010, \\
c_{D^{*} \pi}^{L} & =2 r^{*} \cos (2 \beta+\gamma) \sin \delta-=-0,019 \pm 0,022 \pm 0,019, \\
a_{D^{*} \pi}^{K} & =2 r^{*} \sin (2 \beta+\gamma) \cos \delta=-0,025 \pm 0,020 \pm 0,015, \\
b_{D^{*} \pi}^{K} & =2 r^{\prime} \sin (2 \beta+\gamma) \cos \delta=-0,004 \pm 0,010 \pm 0,011, \\
c_{D^{*} \pi}^{K} & =2 \cos (2 \beta+\gamma)\left(r^{*} \sin \delta-r^{\prime} \sin \delta^{\prime}\right)=-0,002 \pm 0,020 \pm 0,016 .
\end{aligned}
$$

On en déduit la mesure combinée de l'asymétrie de $C P a_{D^{*} \pi}^{L}=2 r^{*} \sin (2 \beta+\gamma) \cos \delta=$ $-0,034 \pm 0,014 \pm 0,009$. L'analyse pose les premiers jalons de la mesure de l'angle $\gamma$ du triangle d'unitarité. Bien que l'on ne puisse pas encore parler d'observation avec la statistique actuelle, ce mode se révèle être très prometteur dans l'avenir pour contraindre $\gamma$.

La méthode de reconstruction partielle a prouvé sa grande efficacité par rapport à une méthode de reconstruction exclusive. La procédure désormais bien établie va maintenant pouvoir être utilisée pour la mesure de la violation de $C P$ dans d'autres modes de désintégrations du $B$, tels que $D^{*} \rho$ ou $D^{*} a_{1}$. En plus d'apporter de nouvelles mesures de $\sin (2 \beta+\gamma)$, l'étude de la violation de $C P$ dans les désintégrations $B^{0} \rightarrow D^{*-} \rho^{+}$pourra permettre de réduire significativement une des systématiques principales de l'analyse, qui est due à notre ignorance de l'amplitude de la violation de $C P$ dans le bruit de fond $D^{*} \rho$. Un autre point qu'il sera important de travailler dans les prochaines années est la connaissance du rapport $r^{*}$, tant au point de vue théorique, qu'expérimental. L'ajout de statistique devrait permettre une mesure plus précise du rapport d'embranchement $\mathcal{B}\left(B^{0} \rightarrow D_{s}^{*+} \pi^{-}\right)$, qui est utilisé pour estimer $r^{*}$. D'autres mesures devraient également contribuer à la détermination de $r^{*}$. Par exemple, la mesure récente de $\mathcal{B}\left(D_{s}^{+} \rightarrow \phi \pi^{+}\right)$ dans $B A B A R$, qui a également utilisé une méthode de reconstruction partielle, a permis d'améliorer la connaissance de $r^{*}$. Cela, couplé à l'augmentation de la statistique dans les prochaines années, semble indiquer qu'il sera possible d'observer une asymétrie de $C P$ significative dans ce mode avec les données de BABAR. 
La valeur mesurée des asymétries de $C P$ est proche de la limite physique, ce qui nous a amené à développer une méthode d'interprétation fréquentiste des résultats, inspirée de la méthode proposée par Feldman-Cousins. On a ainsi pu déterminer un niveau de confiance en fonction de $\sin (2 \beta+\gamma)$, conduisant aux limites $|\sin (2 \beta+\gamma)|>0.35(0,62)$ à $90 \%(68$ $\%)$ de niveau de confiance. Cela permet également de contraindre le sommet du triangle d'unitarité dans le plan $(\rho, \eta)$.

La précision sur la mesure de $\gamma$ devrait s'améliorer de façon notable dans les prochaines années. On pourra alors comparer les mesures de $\gamma$ obtenues en étudiant les désintégrations de $B^{0}$, qui font intervenir le mélange $B^{0} \bar{B}^{0}$, et celles obtenues en étudiant les désintégrations des $B^{ \pm}$, pour lesquelles il n'y a pas de mélange. Une différence significative entre les mesures de $\gamma$ dans ces deux types de désintégrations serait ainsi un indice de nouvelle physique dans le mélange $B^{0} \bar{B}^{0}$.

\section{Alignment du DIRC}

Le code mis en place pour l'alignement du DIRC constitue un package autonome qui permet de recalculer les corrections facilement et régulièrement. La nouvelle table de correction ainsi produite est maintenant utilisée dans la base de données de BABAR depuis le début de l'année 2003. Elle a permis d'améliorer sensiblement la résolution sur l'angle de Cherenkov des photons émis par une particule chargée traversant le milieu radiateur du DIRC. 


\section{Bibliographie}

[1] E. Noether, Invariante variationsprobleme, Nachr. v. d.Ges. d. Wiss. zu Göttingen (1918), p. 235-257.

[2] T. Lee et C. Yang, Phys. Rev. 104 (1956), p. 254.

[3] C. S. Wu, E. Ambler, R. W. Hayward, D. D. Hoppes et R. P. Hudson, Experimental test of parity conservation in beta decay, Phys. Rev. 105 (1957), p. 1413-1414.

[4] D. H. Wilkinson, Parity conservation in strong interactions, Phys. Rev. 109 (1958), p. 1603-1609.

[5] L. Dobrzynski et al, Test of $C P$ and $C$ invariances in $p \bar{p}$ annihilations at $1.2 \mathrm{GeV} / c$ involving strange particles, Phys. Lett. 22 (1966), no 1, p. 105-108.

[6] R. I. Dzhelyadin et al, Search for rare decays of $\eta$ and $\eta^{\prime}$ mesons, and for light Higgs particles, Phys. Lett. 105B (1981), no 2,3, p. 239-241.

[7] S. Barshay, Proposal to test time-reversal invariance in the reactions $\gamma+d \rightleftharpoons n+p$, Phys. Rev. Lett. 17 (1966), no 1, p. 49-53.

[8] D. Sober et al,

Angular distribution of deuteron photodisintegration between 240 and $320 \mathrm{MeV}$,

Phys. Rev. Lett. 22 (1969), no 9, p. 430-433.

[9] CPLEAR Collaboration, A. Angelopoulos et al, PLB 444 (1998), nº 43.

[10] J. H. Christenson, J. W. Cronin, V. L. Fitch et R. Turlay, Evidence for the $2 \pi$ decay of the $K_{2}^{0}$ meson, Phys. Rev. Lett. 13 (1964), p. 138-140.

[11] C. NA48, V. Fanti et al, PLB 465 (1999), no 335.

[12] C. KTeV, A. Alavi-Harati et al, PRL 83 (1999), no 22. 
[13] BABARCOLL,

Direct $C P$ Asymmetry in $B^{0} \rightarrow K^{+} \pi^{-}$Decays,

PRL 93 (2004), nº 131801.

[14] C. Belle, Y. Chao et al,

Evidence for direct $C P$ violation in $B^{0} \rightarrow K^{+} \pi^{-}$Decays,

PRL 93 (1999), no 191802.

[15] W. Pauli,

Exclusion Principle, Lorentz Group and Reflexion of Space-time and Charge,

New York, Mc Graw-Hill (1955).

[16] G. Lüders,

Proof of the $\mathcal{T} C \mathcal{P}$ theorem,

Ann. Phys. 2 (1957), p. 1.

[17] A. D. Sakharov,

Violations of $C P$ invariance, $C$ asymmetry, and baryon asymmetry of the universe, JETP Lett. 4 (1967), p. 24.

[18] R. Aleksan,

Quark Mixing and $C P$ violation,

Technics and Concepts in High Energy Physics X Kluwer Academic Publ. (1999), p. $487-596$.

[19] T. Nakada,

$C P$ violation in $\mathbf{K}$ - and $\mathbf{B}-M e s o n$ Decays,

PSI-PR-91-02 (1991).

[20] Collaboration BABAR, B. Aubert et al,

The BABAR physics book : Physics at an asymmetric $B$ factory,

SLAC Report R-504 (octobre 1998),

édité par P. F. Harrison et H. R. Quinn.

[21] S. L. Glashow,

Partial-symmetries of weak interactions,

Nucl. Phys. 22 (1961), p. 579-588.

[22] S. Weinberg,

A model of leptons,

PRL 19 (1967), no 21, p. 1264-1266.

[23] P. W. Higgs,

Broken symmetries and the masses of gauge bosons,

Phys. Rev. Lett. 13 (1964), no 16, p. 508-509.

[24] N. Cabibbo,

Unitary symmetry and leptonic decays,

Phys. Rev. Lett. 10 (1963), no 12, p. 531-533.

[25] M. Kobayashi et T. Maskawa,

$C P$-violation in the renormalizable theory of weak interaction,

Progr. Theor. Phys. 49 (1973), no 2, p. 652-657. 
[26] L.-L. Chau et W.-Y. Keung,

Comments on the parametrization of the Kobayashi-Maskawa matrix, Phys. Rev. Lett. 53 (1984), nº 19, p. 1802-1805.

[27] L. Wolfenstein,

Parametrization of the Kobayashi-Maskawa matrix,

Phys. Rev. Lett. 51 (1983), no 21, p. 1945-1947.

[28] C. Jarlskog,

Phys. Rev. Lett. 55 (1985), $\mathrm{n}^{\circ} 1039$.

[29] K. Hagiwara et al,

Particle Data Group,

Phys. Rev. D (2004),

Http ://pdg.lbl.gov/pdg.html.

[30] J. Charles et al,

$C P$ Violation and the CKM Matrix : Assessing the Impact of the Asymmetric B Factories, hep-ph/0406184 .

[31] Collaboration BABAR,

BABAR technical design report,

SLAC Report R-457 (mars 1995).

[32] Collaboration BABAR, B. Aubert et al,

The BABAR detector,

Nucl. Instr. Methods A 479 (2002), p. 1-116.

[33] PEP-II, an asymmetric $B$ factory : Conceptual design report,

SLAC Report R-418 (juin 1993).

[34] C. Bozzi et al,

The design and construction of the BABAR silicon vertex tracker,

Nucl. Instr. Methods A 447 (2000), p. 15-25.

[35] D. Barbieri et al,

Silicon sensors for the BABAR vertex tracker : design, electrical tests and production quality control,

Nuov. Cim. 112 A (1999), no 1-2, p. 113-130.

[36] A. Boucham et al,

The BABAR drift chamber project,

Nucl. Instr. Methods A 409 (1998), p. 46-52.

[37] G. Sciolla et al,

The BABAR drift chamber,

Nucl. Instr. Methods A 419 (1998), p. 310-314.

[38] P. Coyle et al,

The DIRC counter : a new type of particle identification device for $B$ factories,

Nucl. Instr. Methods A 343 (1994), p. 292-299.

[39] I. Adam et al,

DIRC, the internally reflecting ring imaging Cherenkov detector of BABAR, 
IEEE Trans. Nucl. Sci. 45 (juin 1998), nº 3, p. 657-664.

[40] I. Adam et al,

An internally reflecting Cherenkov detector (DIRC) : properties of the fused silica radiators,

IEEE Trans. Nucl. Sci. 45 (juin 1998), nº 3, p. 450-455.

[41] P. Bourgeois, M. Karolak et G. Vasseur,

Performance of the photomultiplier tubes used in the DIRC of BABAR : effect of a magnetic field and of helium,

Nucl. Instr. Methods A 442 (2000), p. 105-112.

[42] M. Benkebil, R. Cizeron, S. Plaszczynski, M. H. Schune et G. Wormser,

Water resistant rhodium plated reflectors for use in the DIRC BABAR Cherenkov detector,

Nucl. Instr. Methods A 442 (2000), p. 364-367.

[43] P. Bailly, J. Chauveau, J. F. Genat, J. F. Huppert, H. Lebbolo, L. Roos et B. Zhang,

A 16-channel digital TDC chip with internal buffering and selective readout for the DIRC Cherenkov .

[44] P. Bailly, J. Chauveau, J. F. Genat, J. F. Huppert, H. Lebbolo, L. Roos et B. Zhang,

A 16-channel digital TDC chip,

Nucl. Instr. Methods A 433 (1999), p. 432-437.

[45] G. Manzin,

The BABAR calorimeter,

Nucl. Phys. (Proc. Suppl.) B 78 (1999), p. 454-458.

[46] T. G. O'Connor et al,

Design and testing of the 1.5 Tesla superconducting solenoid for the BABAR detector at PEP-II in SLAC,

IEEE Trans. App. Super. 9 (1999), nº 2, p. 857-851.

[47] F. Anulli et al,

The muon and neutral hadron detector for BABAR,

Nucl. Instr. Methods A 409 (1998), p. 542-546.

[48] R. Santonico et R. Cardarelli,

Development of resistive plate counters,

Nucl. Instr. Methods 187 (1981), p. 377-380.

[49] R. Cardarelli, R. Santonico, A. Di Biagio et A. Lucci,

Progress in resistive plate counters,

Nucl. Instr. Methods A 263 (1988), p. 20-25.

[50] R. Aleksan, I. Dunietz et B. Kayser,

Determining the $C P$-violating phase $\gamma$,

Z. Phys. C 54 (1992), p. 653-659.

[51] R. Aleksan, I. Dunietz, B. Kayser et F. Le Diberder,

$C P$ violation using non- $C P$ eigenstate decays of neutral $B$ mesons,

Nucl. Phys. B 361 (1991), p. 141-165.

[52] M. Gronau et D. Wyler,

Phys. Lett. B 265 (1991), p. 172. 
[53] D. Atwood, I. Dunietz et A. Soni,

Phys. Rev. Lett. 78 (1997), p. 3257.

[54] Collaboration BABAR, B. Aubert et al,

Measurement of the branching fractions and $C P$-asymmetry of $B^{0} \rightarrow$ $D_{(C P)}^{0} K^{-}$decays with the BABAR detector,

Phys. Rev. Lett. 92 (2004), no 202002.

[55] Collaboration BABAR, B. Aubert et al,

Measurement of $C P$-Asymmetries for the Decays $B \rightarrow D_{C P} K^{* \pm}$ with the BABAR Detector,

hep-ex/0408069 .

[56] Collaboration BABAR, B. Aubert et al,

Measurement of the Ratio $\mathcal{B}\left(B^{-} \rightarrow D^{* 0} K^{-}\right) / \mathcal{B}\left(B^{-} \rightarrow D^{* 0} \pi^{-}\right)$and the $C P$ asymmetry of $B^{-} \rightarrow D^{* 0}(C P+) K^{-}$decays with the $B A B A R$ detector, hep-ex/0408060 .

[57] Collaboration Belle,

Study of $B^{ \pm} \rightarrow D_{C P} K^{ \pm}$and $B^{ \pm} \rightarrow D_{C P}^{*} K^{ \pm}$ BELLE-CONF-0443 .

[58] Collaboration BABAR, B. Aubert et al,

Search for $b \rightarrow u$ transitions in $B^{-} \rightarrow \bar{D}^{0} K^{-}$and $B^{-} \rightarrow \bar{D}^{* 0} K^{-}$, hep-ex/0408028 .

[59] Collaboration Belle,

Study of the suppressed decays $B^{ \pm} \rightarrow\left[K^{ \pm} \pi^{ \pm}\right]_{D} K^{ \pm}$at Belle", hep-ex/0412025 .

[60] A. Giri, Y. Grossman, A. Soffer et J. Zuppan,

Phys. Rev. D 68 (2003), no 0504018.

[61] Collaboration BABAR, B. Aubert et al,

Measurement of $\gamma$ in $b^{\mp} \rightarrow d^{(*)} k^{\mp}$ decays with a dalitz analysis of $d \rightarrow$ $k_{s}^{0} \pi^{-} \pi^{+}$, hep-ex/0504039 .

[62] Collaboration Belle,

Measurement of $\phi_{3}$ with dalitz analysis of $b^{ \pm} \rightarrow d^{(*)} k^{ \pm}$decay at belle, hep-ex/0411049, v2 .

[63] R. Sachs (1985),

Enrico Fermi Institute Report, EFI-85-22.

[64] I. Dunietz et R. Sachs,

Asymmetry between inclusive charmed and anticharmed modes in $B^{0}, \bar{B}^{0}$ decay as a measure of $C P$ violation,

Phys. Rev. D 37 (1988), p. 3186.

[65] I. Dunietz,

Clean CKM information from $B_{d}(t) \rightarrow D^{(*) \mp} \pi^{ \pm}$

Phys. Lett. B 427 (1998), p. 179.

[66] A. Soffer et W. Walkowiak,

Probability density function for $C P$ violation and mixing including mistag due to partial reconstruction (2003), 
BABAR analysis document $\mathrm{n}^{\circ} 391$.

[67] M. Baak, R. Cahn, D. Kirkby et O. Long,

Impact of the tag-side interference on time-dependant $C P$ asymmetry measurements using coherent $B^{0} / \bar{B}^{0}$ pairs,

Phys. Rev. D 68 (2003), nº 034010.

[68] K. Hagiwara et al,

Particle Data Group,

Phys. Rev. D 60 (2002).

[69] Collaboration BABAR, B. Aubert et al,

A study of the rare decays $B^{0} \rightarrow D_{s}^{(*)+} \pi^{-}$and $B^{0} \rightarrow D_{s}^{(*)-} K^{+}$,

Phys. Rev. Lett. 90 (2003), no 181803.

[70] Collaboration Belle,

Observation of $D_{s}^{+} K^{-}$and evidence for $D_{s}^{+} \pi^{-}$final states in neutral B decays,

Phys. Rev. Lett. 89 (2002), no 231804.

[71] D. Becirevic,

Heavy Quark Phenomenology from Lattice QCD,

Nucl. Phys. (Proc. Suppl.) 94 (2001), p. 337.

[72] Collaboration BABAR, B. Aubert et al, hep-ex/0408040 .

[73] Collaboration Belle,

Phys. Rev. Lett. 89 (2002), no 231804.

[74] Collaboration BABAR, B. Aubert et al, Phys. Rev. Lett. 90 (2003), nº 181803.

[75] A. Datta et D. London, Phys. Lett. B 584 (2004).

[76] T. Heavy Flavor Averaging Group,

Summer 2004 averages,

Http ://www.slac.stanford.edu/xorg/hfag.

[77] R. Giles et al,

Two-body decays of $B$ mesons,

PRD 30 (1984), no 11, p. 2279-2294.

[78] Collaboration Argus, A. Albrecht et al,

Détermination of the branching ratio for the decay $B^{0} \rightarrow D^{*-} \pi^{+}$,

Phys. Lett. B 182 (1986), no 1, p. 95-100.

[79] Collaboration BABAR, B. Aubert et al,

Measurement of the $B^{0}$ meson lifetime with partial reconstruction of $B^{0} \rightarrow$ $D^{*-} \pi^{+}$and $B^{0} \rightarrow D^{*-} \rho^{+}$decays,

PRD 67 (2003), nº 091101.

[80] R. A. Fisher,

The use of multiple measurements in taxonomic problems,

Annual Eugenics 7 (1936), nº 179.

[81] M. S. Srivastava et E. M. Carter, 
An introduction to applied multivariate statistics (1983),

Amsterdam.

[82] B. T. T. Group,

B tagging in BABAR (2002),

BABAR analysis document $\mathrm{n}^{\circ} 119$.

[83] G. Fox et S. Wolfram,

Event shapes in $e^{+} e^{-}$annihilation 149 (1979), p. 413.

[84] F. James,

MINUIT,

CERN program library D506.

[85] Collaboration Argus, A. Albrecht et al,

Phys. Lett. B 254 (1991), p. 288.

[86] G. Feldman et R. Cousins,

A unified approach to the classical statistical analysis of small signals, Phys. Rev. D 57 (1998), p. 3873-3889.

[87] J. Neyman,

Philos. Trans. R Soc. London Sect. A 236 (1937), p. 333-380.

[88] R. Cousins,

Why isn't every physicist a bayesian?,

Am. J. Phys. 63 (1995), nº 5, p. 398.

[89] Collaboration BABAR, B. Aubert et al,

Improved Measurement of $C P$ Asymmetries in $B^{0} \rightarrow c \bar{c} K^{0(*)}$ Decays, hep-ex/0408127.

[90] Collaboration Belle,

Improved Measurement of $C P$-Violation Parameters $\sin \left(2 \phi_{1}\right)$ and $|\lambda|, \mathbf{B}$

Meson Lifetimes, and $B^{0}-\bar{B}^{0}$ Mixing Parameter $\Delta m_{d}$, hep-ex/0408111 .

[91] Collaboration BABAR, B. Aubert et al,

Ambiguity-Free Measurement of $\cos (2 \beta)$ : Time-Integrated and Time-

Dependent angular Analyses of $B \rightarrow J / \Psi K \pi$, hep-ex/0411016 .

[92] Collaboration Belle,

Studies of $C P$ violation in $B^{0} \rightarrow J / \psi K^{* 0}$ decays, hep-ex/0408104 .

[93] Collaboration BABAR, B. Aubert et al,

Measurement of $C P$-violating parameters in fully reconstructed $B \rightarrow D^{(*)} \pi$

and $B \rightarrow D \rho$ decays,

hep-ex/0408059 .

[94] Collaboration Belle,

Time-Dependent $C P$ Violation Effects in Partially Reconstructed $B^{0} \rightarrow$ $D^{*} \pi$ Decays,

hep-ex/0408106 .

[95] Collaboration Belle, 
Study of $C P$ Violating Effects in Time Dependent $B^{0}\left(\overline{B^{0}}\right) \rightarrow D^{(*) \mp} \pi^{ \pm}$Decays,

Phys. Rev. Lett. 93 (2004), nº 031802. 